\title{
Plasmon-Enhanced Optical Sensing by Engineering Metallic Nanostructures
}

\author{
Peng Zheng \\ West Virginia University, pzheng1@mix.wvu.edu
}

Follow this and additional works at: https://researchrepository.wvu.edu/etd

Part of the Analytical Chemistry Commons, Materials Science and Engineering Commons, Nanoscience and Nanotechnology Commons, and the Optics Commons

\section{Recommended Citation}

Zheng, Peng, "Plasmon-Enhanced Optical Sensing by Engineering Metallic Nanostructures" (2018). Graduate Theses, Dissertations, and Problem Reports. 3710.

https://researchrepository.wvu.edu/etd/3710

This Dissertation is protected by copyright and/or related rights. It has been brought to you by the The Research Repository @ WVU with permission from the rights-holder(s). You are free to use this Dissertation in any way that is permitted by the copyright and related rights legislation that applies to your use. For other uses you must obtain permission from the rights-holder(s) directly, unless additional rights are indicated by a Creative Commons license in the record and/ or on the work itself. This Dissertation has been accepted for inclusion in WVU Graduate Theses, Dissertations, and Problem Reports collection by an authorized administrator of The Research Repository @ WVU.

For more information, please contact researchrepository@mail.wvu.edu. 


\title{
Plasmon-Enhanced Optical Sensing by Engineering Metallic Nanostructures
}

\section{Peng Zheng}

\author{
Dissertation submitted \\ to Benjamin M. Statler College of Engineering and Mineral Resources \\ at West Virginia University \\ in partial fulfillment of the requirements for the degree of \\ Doctor of Philosophy in \\ Materials Science and Engineering \\ Nianqiang Wu, Ph.D., Chair \\ Ever J. Barbero, Ph.D. \\ Yon Rojanasakul, Ph.D. \\ Lisa Holland, Ph.D. \\ Terence Musho, Ph.D. \\ Kostas Sierros, Ph.D.
}

Department of Mechanical and Aerospace Engineering

Morgantown, West Virginia, United States

2018

Keywords: Localized Surface Plasmon Resonance, Optical Sensing, Biosensor, Metallic

Nanostructures, Surface-Enhanced Raman Scattering, Plasmon-Enhanced Fluorescence, Nanopyramid Array, Nanocube, Discrete Dipole Approximation, Finite-Difference Time-Domain 


\section{Abstract \\ Plasmon-Enhanced Optical Sensing by Engineering Metallic Nanostructures \\ Peng Zheng}

The world's booming population projected to reach 10 billion by 2050 causes enormous stresses on environmental safety, food supply, and healthcare, which in return threatens human civilizations. One of the most promising solutions lies at innovating point-of-care (POC) sensing technologies to conduct detection of environmental hazards, monitoring of food safety, and early diagnosis of diseases in a timely and accurate manner. The discovery of surface-enhanced spectroscopy in the 1970s has significantly stimulated research on light-matter interaction which gives rise to enhanced optical phenomena such as surface-enhanced Raman scattering (SERS), plasmon-enhanced fluorescence (PEF), and particularly, they have found enormous applications in optical sensing. To fully exploit surface-enhanced spectroscopy to advance sensing technologies, it requires innovations in the sensor design as well as the plasmonic metallic nanostructures, which is exactly the focus of this dissertation. Owing to their strong capabilities of revealing molecular fingerprints and conducting single molecule analysis, both SERS and PEF have received extensive research interests. Since SERS directly correlates with the local electromagnetic (EM) field enhancement, it is featured by the simplicity in signal amplification. However, high SERS spectral resolution cannot be achieved without a tightly focused laser beam, which compromises the design of SERS-based POC sensing platforms. In contrast, the emission nature of fluorescence makes PEF easily coupled with POC readers, but optimal PEF requires a delicate control of the separation distance between the fluorophore and the nanostructure to minimize fluorescence quenching. SERS and PEF are essentially two complementary techniques and both hold great promise for POC sensing technologies.

In the dissertation, in the first place, two label-free SERS sensors have been developed aiming to reduce the number of elements used in a sensor, which could potentially minimize interference, reduce the cost, and enhance the performance. In this regard, a label-free SERS sensor for mercury ions $\left(\mathrm{Hg}^{2+}\right)$ detection has been developed based on functionalized gold nanoparticles, which employs a small molecule 4-mercaptobenzoic acid to capture mercury ions. A coordination bond formed only in the presence of mercury ions produces a new SERS peak at $374 \mathrm{~cm}^{-1}$, allowing unique detection of mercury ions. The other label-free SERS sensor has been developed for nitrite $\left(\mathrm{NO}_{2}{ }^{-}\right)$detection following the mechanism of Griess reaction based on the plasmonic coupling between gold nanostars and silver nanopyramid arrays. A newly formed azo compound produces at least three characteristic SERS peaks at $1140 \mathrm{~cm}^{-1}, 1389 \mathrm{~cm}^{-1}$, and $1434 \mathrm{~cm}^{-1}$, which allow a highly specific detection of nitrite. While label-free SERS sensing has proved effective to enhance the performance, the need for a tightly focused laser beam hinders SERS from being easily coupled with POC readers for rapid signal readout. 
To address this limitation of SERS, on-chip PEF sensors have been developed, which can be inserted into POC readers for rapid signal readout. Optimizing PEF usually requires a delicate control of the separation distance between the fluorophore and the nanostructure to balance the excitation and emission enhancement which have different distance dependence. In addition to the separation distance, scattering has been found to be strongly correlated with quantum efficiency enhancement, which has been established as another tuning parameter in optimizing PEF. By making PEF work in the near-infrared (NIR) biological transparency window, the strength of PEF is further manifested by its compatibility with biological matrix featured as low background interference and high penetration depth. As a proof of concept, a NIR fluorescent biosensor has been developed for detection of traumatic brain injury biomarker in the blood plasma. The selection of a gold nanopyramid array pattern as the sensing platform not only generates intense localized EM field for the excitation enhancement, but also allows all the tests to be conducted using a POC fluorescence reader.

While noble metals such as gold and silver are often used in developing sensing technologies as they support strong localized surface plasmon resonance (LSPR), it remains an open question as whether they could be replaced by alternative inexpensive metals such as copper and aluminum without compromising the performance. The discovery of a strong and sharp LSPR on copper nanoparticles when the shape is made cubic strongly suggests this possibility. By means of a numeric and theoretical study, it is found that the observed LSPR on copper nanocubes originates from the corner mode which survives damping as it is spectrally separated from the interband transitions. Compared to the dipole mode of a gold nanosphere of the same volume, a copper nanocube displays a comparable extinction coefficient but a local EM field enhancement 7.2 times larger. Furthermore, a film-coupled copper nanocube system has been designed for plasmonenhanced NIR fluorescence. Because of the coupling between the copper nanocube and the underlying film, a plasmonic cavity mode is generated and featured as a spectrally tunable LSPR and an intense local EM field. By tailoring the resonance to the NIR wavelength region, the filmcoupled copper nanocube system has been demonstrated to support a large NIR fluorescence enhancement owing to the strong excitation enhancement and the quantum efficiency enhancement. 
For Mr. Hui Luo, my junior middle school teacher

Thanks for your powerful graduation quote: you will excel in 20 years! 


\section{Acknowledgements}

First and foremost, my sincere gratitude goes to my advisor Prof. Nianqiang Wu for his continuous support and guidance for my $\mathrm{PhD}$ dissertation, for his motivation, patience, enthusiasm, and profound knowledge since I joined the group in August 2011.

Besides my advisor, I would like to express my sincere thanks to my $\mathrm{PhD}$ dissertation committee: Prof. Ever J. Barbero, Prof. Yon Rojanasakul, Prof. Lisa Holland, Prof. Terence Musho, and Prof. Kostas Sierros for their valuable time, encouragement, and insightful advice.

My sincere thanks also go to all my labmates I have worked with at West Virginia University: Prof. Ming Li, Prof. Scott Cushing, Dr. Hui Yang, Sujan Kasani, Joseph Bright, Botong Liu, Prof. Haibin Tang, Jennifer Boryczka, Kathrine Curtin, Dr. Xuefei Gao, Prof. Rui Li, Dr. Jiangtian Li, Dr. Honglei Gou, Prof. Mingjia Zhi, Dr. Fanke Meng, Jinlong Yan, Chengchen Xiang, Prof. Zhenhuan Zhao, Israa A Zaidan, Savan Suri, Prof. Anveeksh Koneru, Shimeng Hao, and Yang He. Were it not for their support and encouragement, I could not have enjoyed so much fun in the past years.

I would also like to express my sincere thanks to Dr. Qiang Wang, Dr. Kolin Brown, Dr. Weiqiang Ding, Dr. Marcela Redigola, Dr. Harley Hart, and Dr. Huiyuan Li from WVU Shared Research Facilities for technical support in my research.

My thanks also go to my friends at WVU: Dr. Zhengjun Wang, Dr. Steve Man Chio Tang, Dr. Disheng Chen, Dr. Bingyi Cui, Dr. Tuhua Zhong, Dr. Jianbo Sun, Dr. Kuijun Li, Dr. Xaomeng Li, Alejandro Mejia, Javier Cabrera, Peng Zhang, Changle Jiang, Fuming Wang, Jinbei Huang, and Dr. Mingyuan Wei. My life would have been very dull were not for soccer we played together.

My special thanks along with my deepest love goes to my wife Xiaorui Tong and my daughter Emma Audrey Zheng. Since 2007 when I was so fortunate to meet my wife, she has always been standing as the person in my life who gives me the most understanding, shows me the strongest support, and trusts me the most unconditionally whatever I am pursuing.

Last but not the least, I would like to express my sincere thanks and love to my family: my aging parents and my married elder sister. They are the most ignored persons in my life during my schooling years but they are also the very persons who offer me the strongest sense of belongings, security, and hope however far away I am staying from home.

Peng Zheng

West Virginia University

November 2018 


\section{Contents}

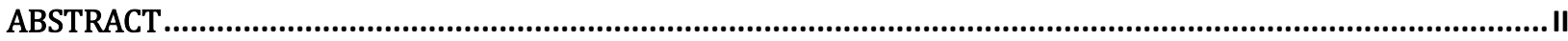

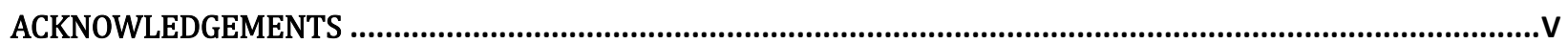

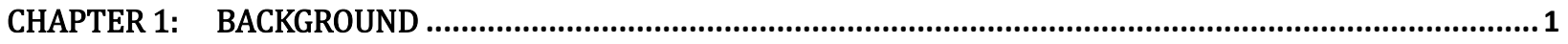

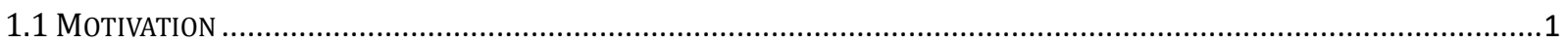

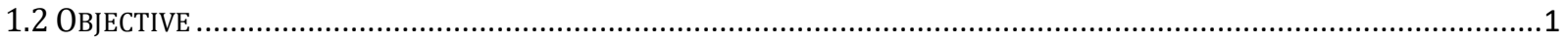

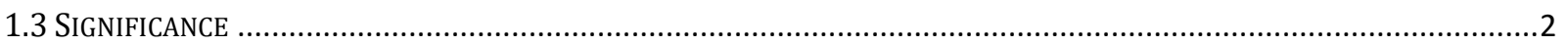

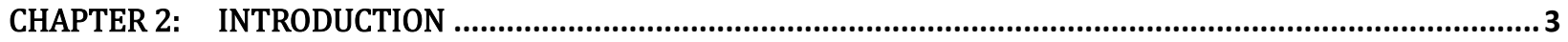

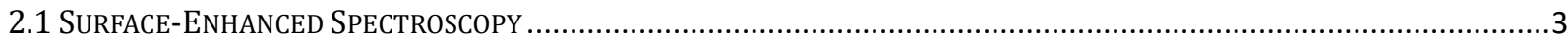

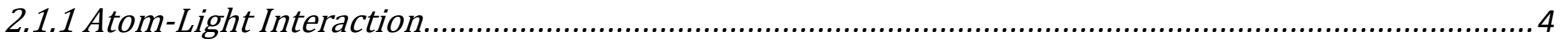

2.1.2 Raman Scattering: Fourth Power Rule ...................................................................................

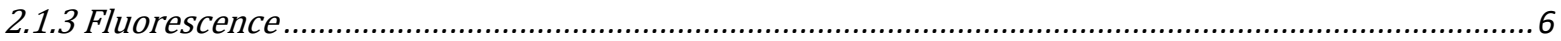

2.1.4 Fluorescence Modification by Atom-Cavity Interaction ................................................................ 7

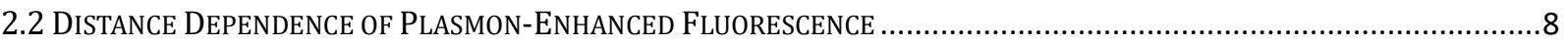

2.3 PuRcell EfFect on Plasmonic Metallic Nanostructures …..................................................................

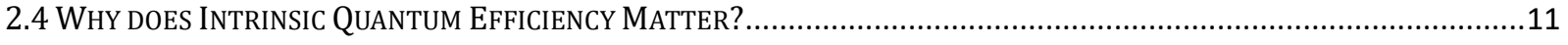

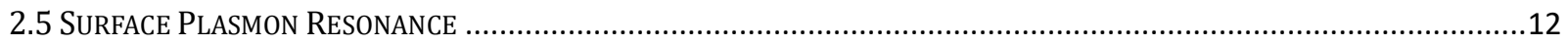

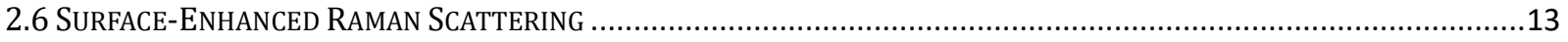

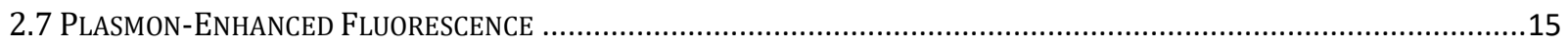

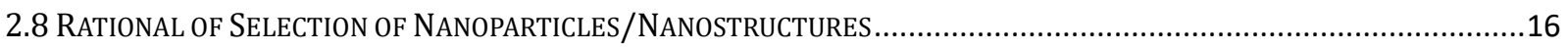

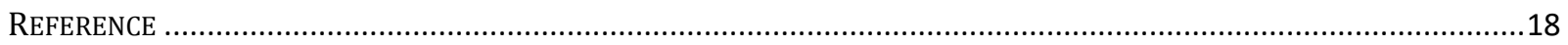

CHAPTER 3: PLASMONIC NANOSTRUCTURES FOR SURFACE-ENHANCED RAMAN SCATTERING SENSING ... 21

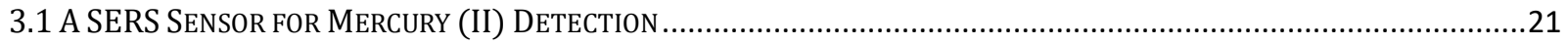

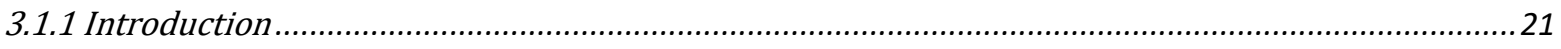

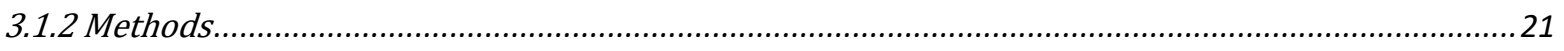

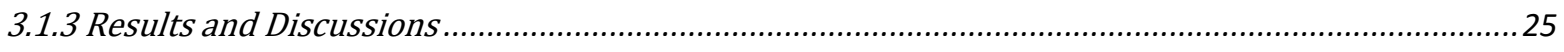

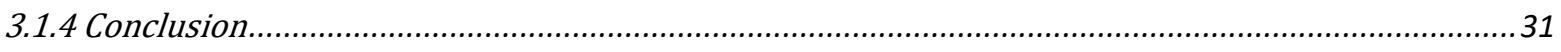

Reference

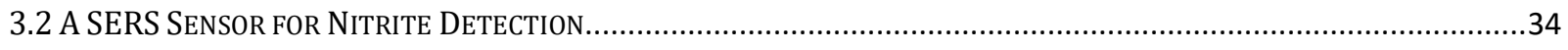

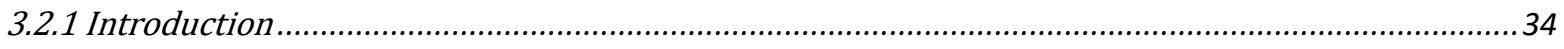

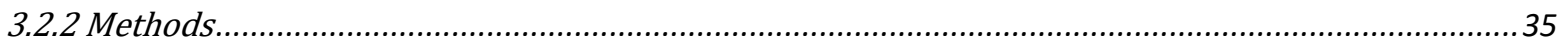

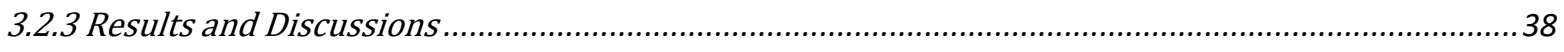

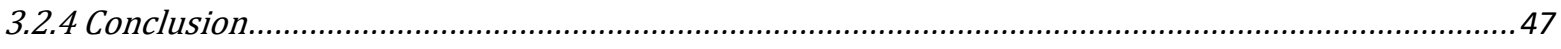

Reference

CHAPTER 4: $\quad$ PLASMON-ENHANCED FLUORESCENCE FOR SENSING................................................................5

4.1 EluCidating the Role of SCATtering In PlaSmon-EnHANCEd FluoreSCENCE ............................................53 


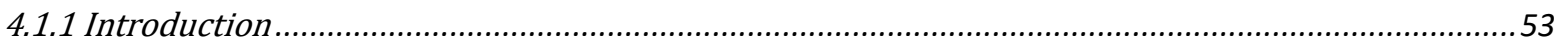

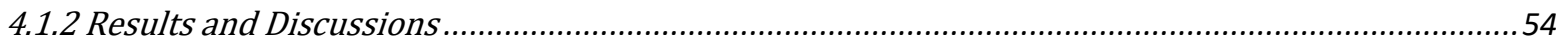

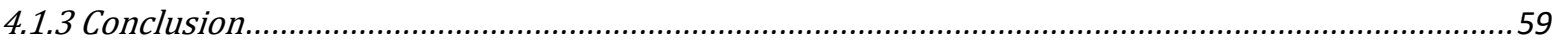

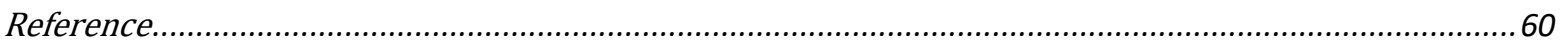

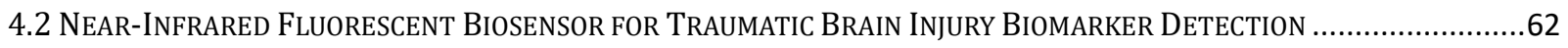

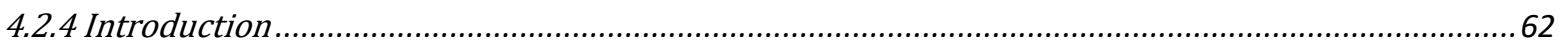

4.2.5 Methods

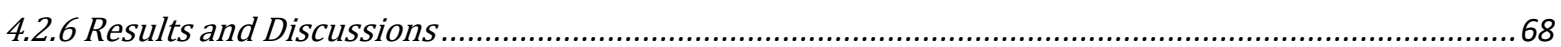

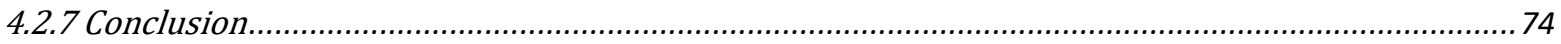

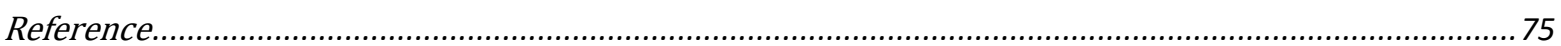

CHAPTER 5: $\quad$ INEXPENSIVE PLASMONIC COPPER MATERIALS ........................................................................ 79

5.1 LOCALIZED SURFACE PLASMON RESONANCE OF COPPER NANOCUBES.........................................................................

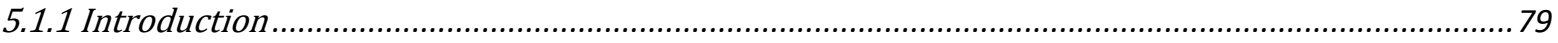

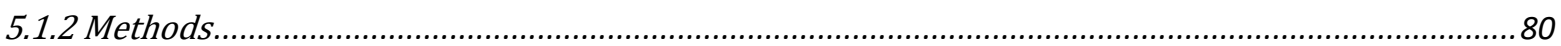

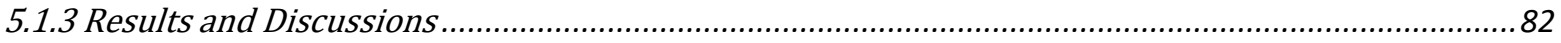

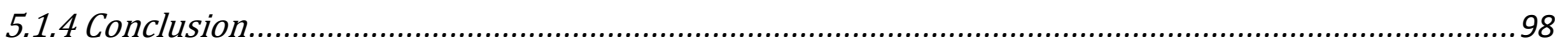

Reference

5.2 FILM-COUPLED COPPER NANOCUBE FOR NEAR-INFRARED FLUORESCENCE ENHANCEMENT ......................................103

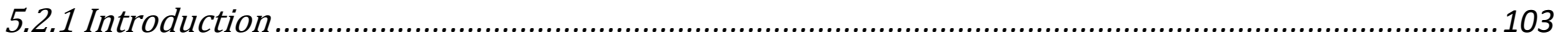

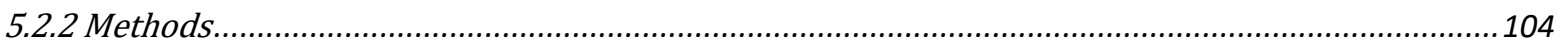

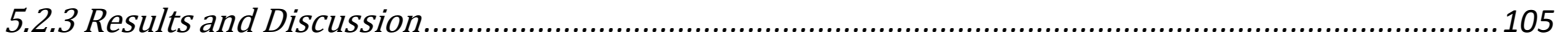

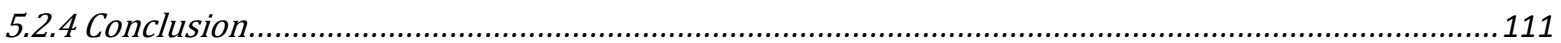

Reference

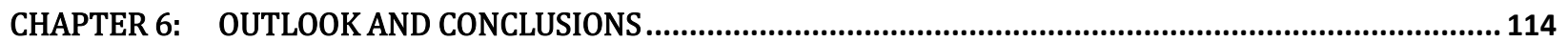

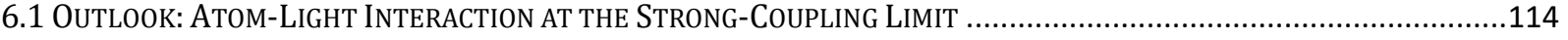

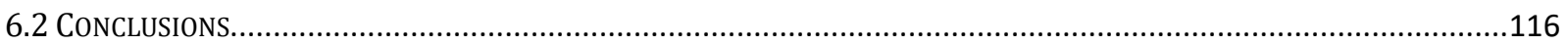

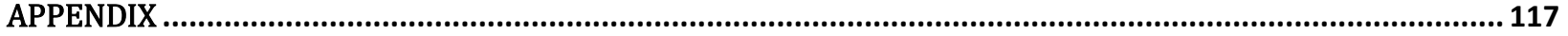

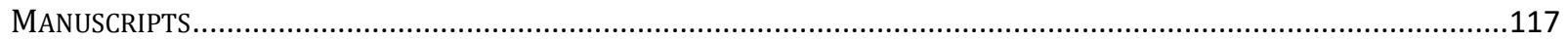

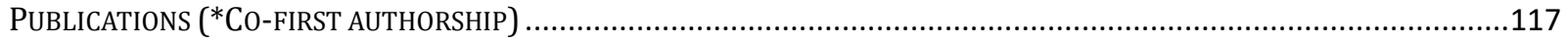




\section{Chapter 1: Background}

\subsection{Motivation}

Human civilizations have been around for about 6,000 years. Since industrialization in the $1800 \mathrm{~s}$, the resilience of human to Earth has improved dramatically. However, after entering $21^{\text {st }}$ century, humanity comes to a crossroad never seen before. The world's population has reached 7.6 billion in 2017 and is projected to reach 10 billion by 2050. The booming population causes enormous stresses on environmental safety, food supply, and healthcare, which in return threaten human civilizations. The three sectors, i.e. environmental safety, food supply, and healthcare, make up one of the most important intercorrelated systems on earth. While environmental safety and a steady supply of food are essential to ensure the welfare of humans, social stability benefited from the welfare would significantly enhance environmental and food safety.

However, the welfare of humans is at risk. Mining and motor vehicle emission contributes a major source of toxic heavy metals, which ends up in aquatic system. Widespread utilization of fertilizers in agriculture produces excessive nutrients, which imposes a far-reaching influence on the sustainable supply of food. Diseases, especially cancers and occasional outbreaks of infectious diseases impose grave threats to human beings. These challenges not only need to be addressed individually, but also as a system thanks to the intercorrelation nature. Therefore, it is imperative to come up with effective solutions to address all these issues associated with the three sectors.

\subsection{Objective}

A lesson learnt from past crisis in human history alludes to preventative measures. The objective of the dissertation is thus to develop sensing systems which could serve as preventative measures to address issues associated with environmental and food safety as well as healthcare. Specifically, the proposed sensing systems shall be able to conduct monitoring of environmental and food safety as well as conduct health diagnosis. To meet the needs, the following objectives have been identified as of paramount importance:

- The proposed sensing systems shall be adaptive. They shall be able to conduct sensing of elements relevant to the environmental and food safety as well as healthcare;

- They shall address limitations of existing approaches, such as sensitivity, selectivity, antiinference, rapidity, cost-effectiveness, and user-friendliness;

- They shall have the potential to be integrated into field-deployable portable devices. 


\subsection{Significance}

With an expanding knowledge of challenges surrounding humans, there are more stringent requirements for sensing systems. However, existing sensing systems such as chromatographyand immunoassay-based approaches cannot meet all the requirements. This gap prompts exploration of alternative innovative solutions. The proposed sensing systems are expected to address this gap by innovations at transforming the capabilities of existing sensing systems or at developing alternative sensing mechanisms. The proposed research is significant in that:

- It refreshes understanding of the capability and limitations of existing sensing systems;

- It explores possibility of transforming and innovating existing sensing capabilities to address challenges surrounding humans;

- It innovates sensing mechanisms to help adapt sensing systems in complex settings, which is otherwise unachievable. 


\section{Chapter 2: Introduction}

\subsection{Surface-Enhanced Spectroscopy}

The discovery of extraordinarily enhanced Raman signal on a roughened silver film over four decades ago has stimulated extensive research interests in surface-enhanced spectroscopy, such as surface-enhanced Raman scattering (SERS), plasmon-enhanced fluorescence (PEF), tip-enhanced Raman scattering (TERS), surface-enhanced hyper Raman scattering (SEHRS), just to name a few. ${ }^{1-6}$ Surface-enhanced spectroscopy employs surface plasmon resonance, which is the coherent oscillation of free electrons at the interface between any two materials with opposite signs of the real part of the dielectric function, to improve both the in- and out-coupling of photons with a molecule or a molecule-like emitter, such as semiconductor quantum dots. As a result, the rate of absorption and emission processes can be modified by orders of magnitude.

Acting as the basis of plasmon-enhanced optical sensing, surface-enhanced spectroscopy has dramatically transformed sensing technologies. It not only advanced the performance (especially sensitivity) never seen before and miniaturized the sensing device unachievable previously, but also opened opportunities for completely new functionalities. Those concepts seemingly far away two decades ago such as single-molecule detection and ultramicroscopy are gradually becoming a reality. The miniaturization of sensing devices thanks to the advancement of nanofabrication technologies has made it possible to develop highly integrated nanosensors. Impressively, new functionalities are gradually added to these sensing devices by bridging with optoelectronics, which allows digitalized and programmable sensing. It is not exaggerating that surface-enhanced spectroscopy is quietly making a transformational impact on the interdisciplinary field of analytical chemistry, nano-optics, materials science, and physics. Recently, surface-enhanced spectroscopy has found enormous applications in environment, agriculture, healthcare, and national security. The miniaturization nature also makes it highly promising for development of point-of-care sensing technologies.

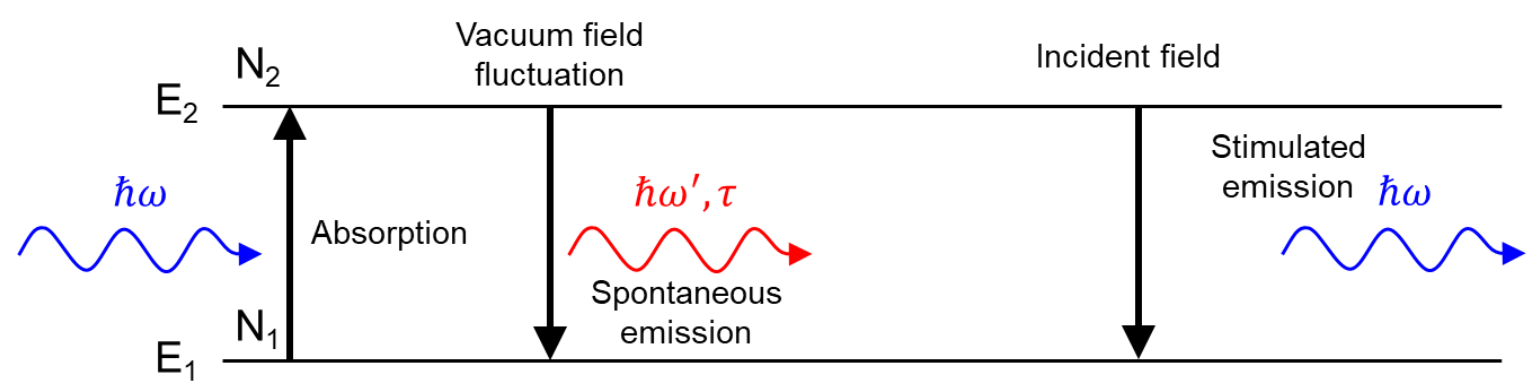

Figure 2.1 Two-level approximation for atom-light interaction. 


\subsubsection{Atom-Light Interaction}

To understand surface-enhanced spectroscopy, it is necessary to look at spectroscopy in the first place. Spectroscopy originates from atom-light interaction. However, the wave nature of light makes it very difficult for an atom to absorb light due to the intrinsic size mismatch. Just consider an atom of $\sim 0.1$ to $0.5 \mathrm{~nm}$ versus the visible light wavelength of $\sim 500$ to $700 \mathrm{~nm}$. The reason why light could still be absorbed is because of its particle nature. A direct consequence is that the atomic absorption cross section is typically very small. This is the reason why it is necessary to improve the absorption cross section by means of surface-enhanced spectroscopy. In the meanwhile, once light is absorbed, it would take a considerable amount of time for the atom to emit a photon. The time taken is called lifetime of the excited atomic state and could range from picoseconds $\left(10^{-12} \mathrm{~s}\right)$ to nanoseconds $\left(10^{-9} \mathrm{~s}\right)$ for a typical photoluminescence event. The lifetime is a very important parameter, as it decides how long the atom could stay excited before returning to the ground state by emitting a photon. Usually, longer lifetime makes the atom more likely to interact with nearby species, ending up being less likely to emit a photon. Since emission spectrum is the key to numerous spectroscopy, it is necessary to bring the excited atom back to the ground state radiatively as soon as possible.

Atom-light interaction can be represented by a simplified two-level atomic system as shown in Figure 2.1. The atom is initially at the ground state $E_{1}$ with an electron population of $N_{1}$. Under incident illumination, only the photons with an angular frequency $\omega$ meeting the condition $\hbar \omega=$ $E_{2}-E_{1}$ will be absorbed, with the atom making an upward transition to the excited state $E_{2}$ and populated with $N_{2}$. The timescale for the absorption process is in the order of $10^{-15} \mathrm{~s}$. Under the perturbation of vacuum field, the excited atom has the tendency to transit back to the ground state $E_{1}$ and at the same time emit a photon. However, this is a spontaneous process with photons randomly emitted independent of the incident field and made possible only because of the perturbation of the vacuum field. The timescale for the spontaneous emission is in the order of $10^{-}$ ${ }^{12} \mathrm{~s}$ to $10^{-9} \mathrm{~s}$. Such a lifetime is usually long enough for the excited atom to participate various catalytical reactions by transferring its energy to nearby species rather than emitting a photon. This is one of the reasons why fluorescence can be quenched.

In an extreme case, if the incident field is extraordinarily strong, not only absorption but also emission could be stimulated. The stimulated emission is a highly coherent process and inherits all the features from the incident field. It also acts as the basis of laser, or light amplification by stimulated emission of radiation. 


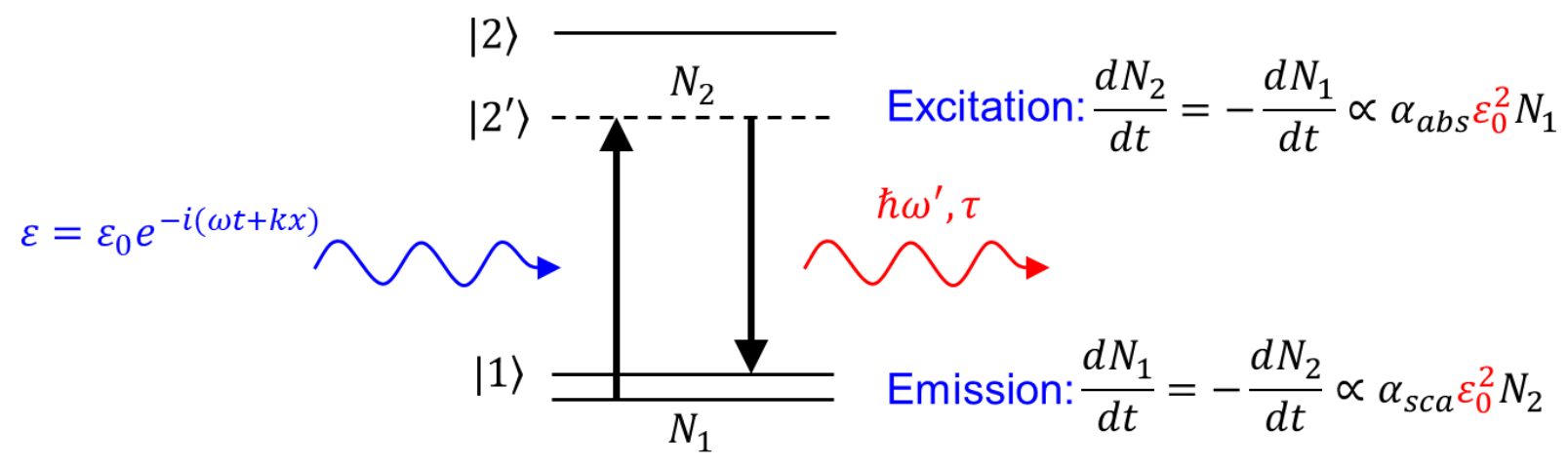

Figure 2.2 Schematic Raman scattering.

\subsubsection{Raman Scattering: Fourth Power Rule}

One of the important consequences of atom-light interaction is Raman scattering, which is an energy Stokes-shifted process, as shown in Figure 2.2. Initially, the atom is excited from a specific vibrational level at the ground state to a virtual excited state (although it could also be a real electronic state, which gives resonant Raman scattering). During de-excitation process, the atom makes a downward transition back to the ground state but at a different vibrational level. This energy difference, usually tiny, is what is analyzed in Raman spectroscopy and features as a Raman shift. If ignoring the tiny energy difference during excitation and emission, the efficiency of Raman scattering scales with the fourth power of the incident field. Raman scattering is an intrinsically inefficient process. It occurs once out of millions of scattering events. Thus, it almost always needs to be enhanced by surface-enhanced spectroscopy. Fortunately, the fourth-power rule provides a straightforward route to improve the efficiency of Raman scattering and acts as the basis for the electromagnetic enhancement mechanism of SERS. Therefore, optimizing SERS falls on engineering plasmonic metallic nanostructures to support strong local fields.

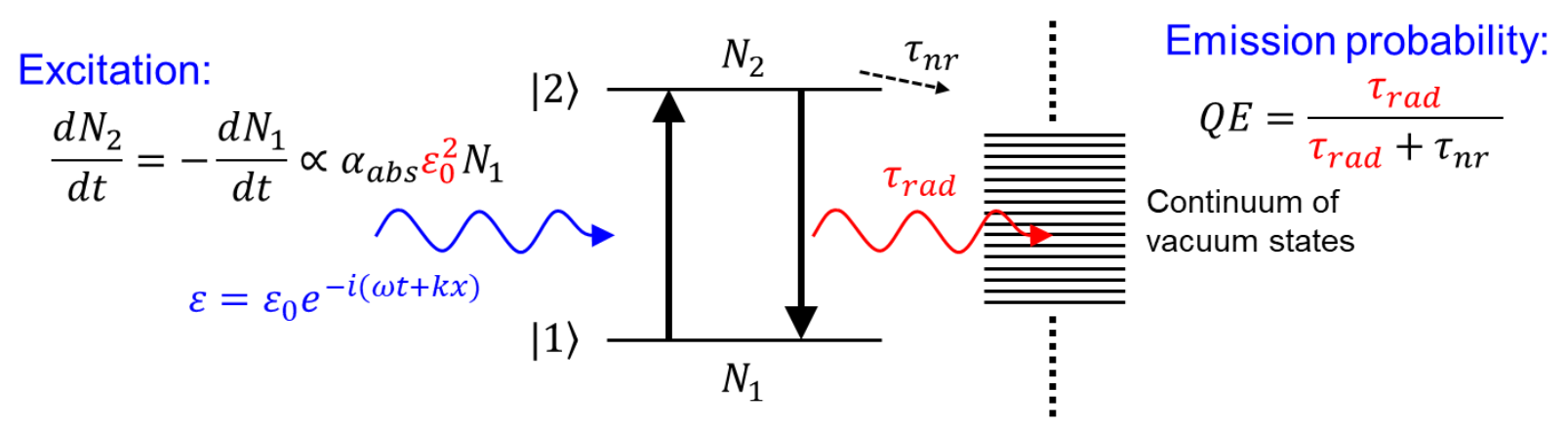

Figure 2.3 Schematic fluorescence process. 


\subsubsection{Fluorescence}

Another important consequence of atom-light interaction is fluorescence, which has a similar excitation process except that the atom makes an upward transition to a real electronic energy state. However, the emission process is different. A schematic fluorescence process is shown in Figure 2.3. Since the atom will spend a considerable amount of time at the excited state before making a downward transition back to the ground state, it has a good chance of interacting with nearby species and unavoidably lose part of its energy, which contributes to non-radiative decay. The radiative process during atomic de-excitation under the perturbation of vacuum field is responsible for the fluorescence emission where photons are emitted to the far field by coupling to the continuum of vacuum states in free space. The spontaneous emission nature makes fluorescence a random and an unregulated process. The emission probability for an excited atom is often quantified by the ratio of the radiative to the overall decay rate and termed as quantum efficiency. Generally, fluorescence is a much more efficient process than Raman scattering although fluorescence could be quenched while Raman scattering would not. Typical fluorescence cross sections are in the order of $10^{-20} \mathrm{~m}^{2}$ as compared to $10^{-33} \mathrm{~m}^{2}$ for non-resonant Raman scattering. In addition, visible fluorophores usually have a much higher quantum efficiency than near-infrared counterparts. That's because the narrower energy levels for near-infrared fluorophores make it more likely for energy transfer to happen, ending up with a much higher non-radiative decay rate.

Unlike Raman spectroscopy where the efficiency of Raman scattering scales with the fourth power of excitation field, fluorescence requires a delicate balance between excitation enhancement and emission probability. While the excitation enhancement is the same as that of Raman scattering which scales with the square of the incident field, an optimized emission needs non-radiative decay to be suppressed in order to minimize fluorescence quenching. The excitation enhancement and emission probability have different distance dependence. Therefore, they cannot be optimized simultaneously. Instead, a compromise must be made. This is also the reason why the fluorescence enhancement is a distance-dependent phenomenon and why it is important to study surfaceenhanced spectroscopy to optimize fluorescence. Therefore, it is necessary to engineer plasmonic metallic nanostructures which not only support strong local fields, but also can improve the emission probability. 

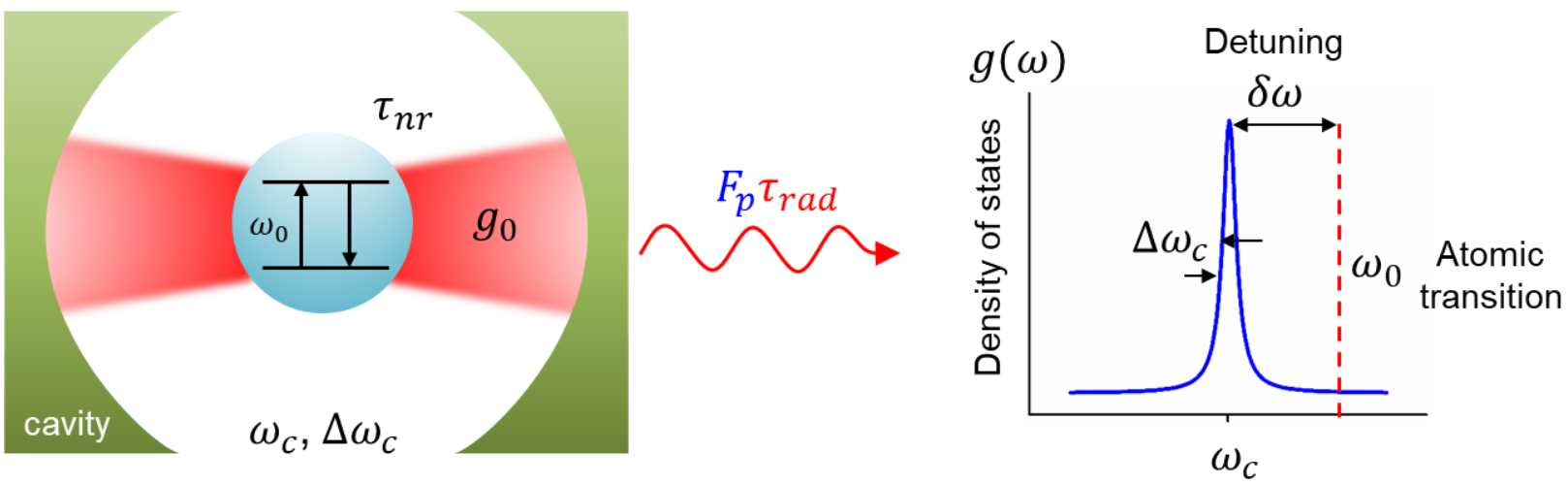

Figure 2.4 Atom-cavity interaction (left) and density of states function (right).

\subsubsection{Fluorescence Modification by Atom-Cavity Interaction}

The mechanism of surface-enhanced spectroscopy can be understood by considering the interaction between an optical cavity and an atom confined inside, as shown in Figure 2.4. It is worth mentioning that an optical cavity is a generalized concept and can represent any system supporting optical resonance, such as photonic crystals and plasmonic nanostructures. Now consider a single-mode cavity with a resonant frequency $\omega_{c}$ and linewidth $\Delta \omega_{c}$, and an atomic transition frequency $\omega_{0}$. Being inside the cavity, the atom experiences an excitation enhancement which is made possible by the enhanced local electric field owing to optical confinement. The electric field enhancement can be quantified by the quality factor $Q=\frac{\omega_{c}}{\Delta \omega_{c}}$. In the meanwhile, the atom can also experience radiative decay modification due to the modified local density of optical states by the cavity. The modification of the local density of optical states can be quantified by the Purcell factor $F_{P}$ given by

$$
F_{P}=\frac{3 Q\left(\frac{\lambda}{n}\right)^{3}}{4 \pi^{2} V_{0}} \frac{\Delta \omega_{c}^{2}}{4 \delta \omega^{2}+\Delta \omega_{c}^{2}}
$$

where $\lambda$ is the incident wavelength, $n$ is the refractive index inside the cavity, $V_{0}$ is the volume of the cavity, and $\delta \omega$ is the detuning defined as $\delta \omega=\omega_{0}-\omega_{c}$. The atom will experience the most radiative decay enhancement quantified by a large Purcell factor $F_{P}$ when the detuning $\delta \omega$ approaches zero, i.e. the atomic transition in resonance with the optical cavity. The consequence is that the quantum efficiency will be modified from

to

$$
Q E_{0}=\frac{\tau_{\text {rad }}}{\tau_{\text {rad }}+\tau_{n r}}
$$




$$
Q E^{\text {new }}=\frac{F_{P} \tau_{\text {rad }}}{F_{P} \tau_{\text {rad }}+\tau_{n r}}
$$

provided that the atomic intrinsic non-radiative decay rate $\tau_{n r}$ is unaffected and that no any other new non-radiative decay channels are introduced, which is a reasonable approximation.

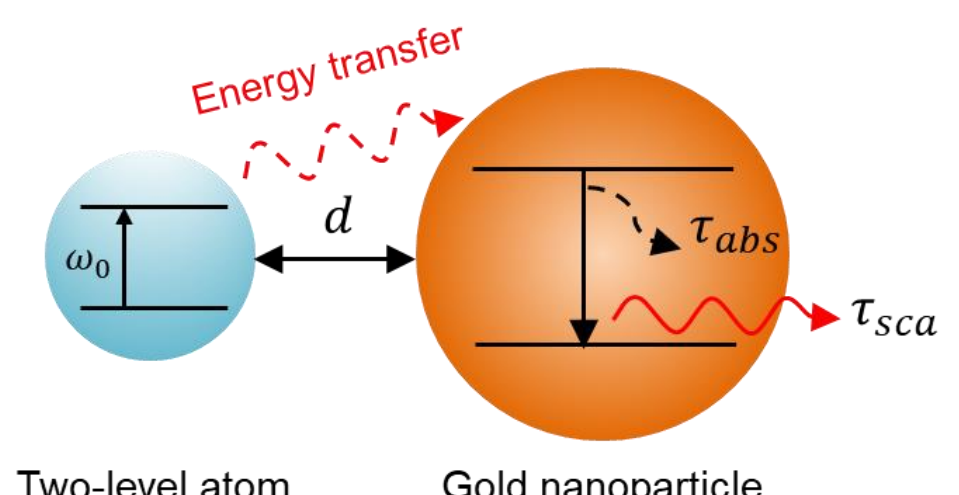

Figure 2.5 Distance dependence of plasmon-enhanced fluorescence.

\subsection{Distance Dependence of Plasmon-Enhanced Fluorescence}

Till now, the distance between the atom and the cavity has not been explicitly discussed since the local field the atom experiences inside the cavity is uniform. Suppose an open optical cavity, i.e. a gold nanoparticle, and a two-level atom separated by a distance of $d$ from it, as shown in Figure 2.5. In this case, the atom-cavity separation distance matters. It is not difficult to see that the local field the atom experiences decays radially from the gold nanoparticle. The way in which the local field decays with respect to the separation distance is consequential. It not only modulates the excitation enhancement which scales with the square of the local field, but also modifies the atomic transition rates.

The distance dependence of the excitation enhancement is related to the decaying profile of the local field for the gold nanoparticle. As the local field of the point dipole-like gold nanoparticle has $\frac{1}{d^{3}}$ dependence, the excitation enhancement would then have $\frac{1}{d^{6}}$ dependence. The distance dependence of the emission enhancement is more complex but can be understood by considering the following scenarios.

For $d=0$ when the atom touches the gold nanoparticle, charge transfer leads to almost complete fluorescence quenching.

For $d \approx d_{0}$, the atom-cavity interacts in the Forster Resonance Energy Transfer (FRET) 
regime where both the atom and the gold nanoparticle can be modelled as point dipole and they interact through dipole-dipole interaction. The Foster distance $d_{0}$ is defined as the separation distance where the FRET efficiency is $50 \%$. The FRET efficiency is given as

$$
E_{F R E T}=\frac{1}{1+\left(\frac{d}{d_{0}}\right)^{6}}
$$

Given the large difference of the timescale between the atomic spontaneous emission $\left(10^{-12} \mathrm{~s}\right.$ to $\left.10^{-9} \mathrm{~s}\right)$ and the plasmon dephasing $\left(10^{-15} \mathrm{~s}\right)$, the excited atom would rapidly de-excite through plasmon decay channels. If absorption dominates the plasmon decay, the excited atom would primarily de-excite through heat dissipation and ends up quenching fluorescence. Otherwise, decay of the excited atom would be dominated by radiative emission to the far field through the plasmon scattering and simultaneously enhanced by the plasmon. In other words, the direction for the quantum efficiency modification in this regime is decided not only by the Purcell effect, but also by the competition between the absorption and scattering on the plasmonic gold nanoparticle, which will be discussed in Chapter 4.1.

For $0<d<d_{0}$, although the FRET efficiency is close to unit, the breakdown of the pointdipole approximation for the gold nanoparticle indicates that non-radiative higher-order plasmon modes dominate, which lead to strong fluorescence quenching.

For $d>d_{0}$, the atom-cavity interacts in the Purcell regime. Owing to a drastic decrease of the FRET efficiency between the excited atom and the gold nanoparticle, the atom would be less likely to experience absorption-induced quenching. Instead, the increase of the local density of optical states at the plasmon resonance frequency would improve the radiative decay rate for the excited atom, ending up improving the quantum efficiency despite at the expense of compromising the excitation enhancement.

Given the complex distance dependence, optimizing PEF requires a delicate control of the atom-cavity separation distance to balance the excitation enhancement and the quantum efficiency, where an optimized separation distance usually occurs in the range of 10 to $20 \mathrm{~nm}$. 


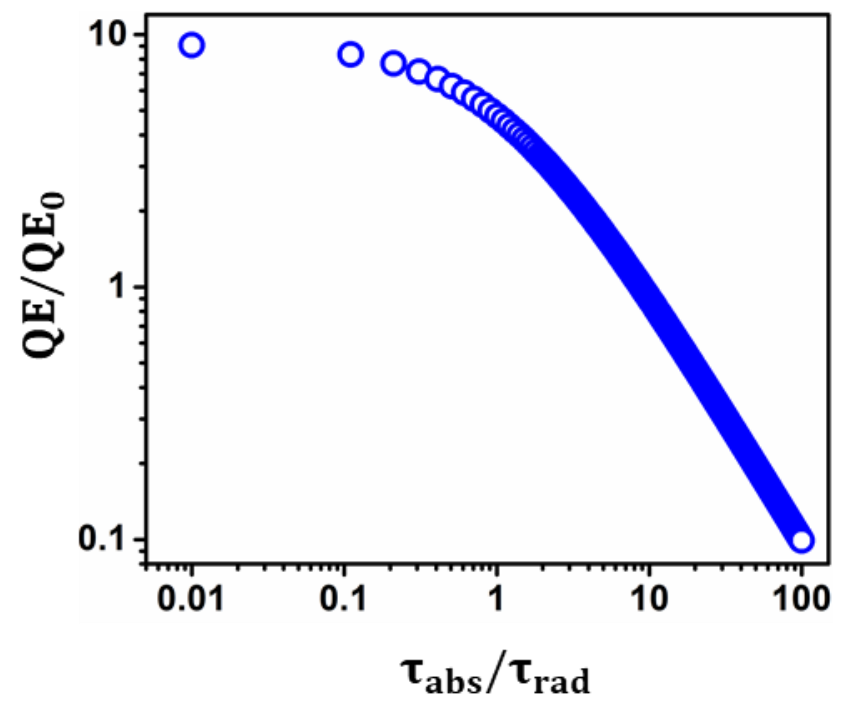

Figure 2.6 Quantum efficiency modification due to the new non-radiative decay channels introduced by the absorption of the optical cavity. In this figure, it is assumed that $Q E_{0}=0.1, F_{P}=100$, and

$$
E_{\text {FRET }}=100 \% \text {. }
$$

\subsection{Purcell Effect on Plasmonic Metallic Nanostructures}

While it is generally true that the imaginary part of the dielectric function for dielectric nanostructures is close to zero and barely contributes to any absorption-induced non-radiative decay, it is a different story for plasmonic metallic nanostructures, which are featured by a large number of free electrons and thus a large imaginary part of the dielectric function. Consequently, the absorption of plasmonic metallic nanostructures opens a new non-radiative decay channel represented by $\tau_{a b s}$. As absorption-induced non-radiative decay would occur only when there is energy transfer between the excited atom and the plasmonic metallic nanostructure, the absorptioninduced non-radiative decay rate would be modified by the FRET efficiency. Therefore, a more generalized representation of the quantum efficiency can be given by

$$
Q E=\frac{F_{P} \tau_{r a d}}{F_{P} \tau_{r a d}+\tau_{a b s}+\tau_{n r}}
$$

By combining Equation (2.2) and Equation (2.5), the quantum efficiency enhancement is obtained and given by

$$
\frac{Q E}{Q E_{0}}=\frac{F_{P}}{F_{P} Q E_{0}+\frac{\tau_{a b s}}{\tau_{r a d}} Q E_{0}+\left(1-Q E_{0}\right)}
$$

From Equation (2.5), if the normalized absorption-induced non-radiative decay $\frac{\tau_{a b s}}{\tau_{\text {rad }}}$ is 
significant, the quantum efficiency is not necessarily improved by a large Purcell factor. Instead, it could be reduced. In Figure 2.6, it is assumed that the intrinsic quantum efficiency is 0.1 and the Purcell factor is 100. The quantum efficiency modification is shown to be dependent on the normalized absorption-induced non-radiative decay rate $\frac{\tau_{a b s}}{\tau_{\text {rad }}}$. For plasmonic metallic nanostructures dominated by absorption, a considerable amount of energy would be dissipated as heat due to electron-electron collisions. Therefore, the Purcell factor is not always an appropriate quantification for the modification of the quantum efficiency, especially when high-loss materials are involved.

It is worth mentioning that in Equation (2.6), the Purcell factor $F_{P}$ and the term $\frac{\tau_{a b s}}{\tau_{r a d}}$ can be directly calculated in commercial simulation software, such as Lumerical FDTD. While the Purcell factor $F_{P}$ quantifies the normalized radiative decay rate and can be obtained from the power monitor group, the term $\frac{\tau_{a b s}}{\tau_{\text {rad }}}$ basically quantifies the normalized non-radiative decay rate owing to Ohmic losses and can be obtained from the power monitor group around the atomic dipole-gold nanoparticle system. Therefore, the quantum efficiency modification could be directly calculated by FDTD.

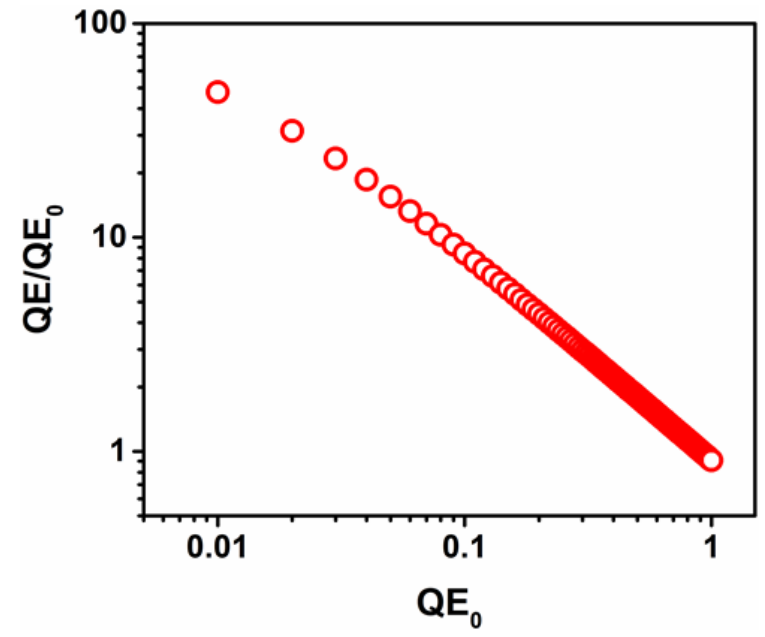

Figure 2.7 Dependence of the quantum efficiency modification $Q E / Q E_{0}$ on the intrinsic quantum efficiency $Q E_{0}$. In this figure, it is assumed that $\tau_{a b s}=0.1 \tau_{\text {rad }}, F_{P}=100$, and $E_{F R E T}=100 \%$.

\subsection{Why does Intrinsic Quantum Efficiency Matter?}

Another point worth mentioning is that an atom with a small intrinsic quantum efficiency is more likely to experience quantum efficiency enhancement. It can be understood from Equation (2.6). A small intrinsic quantum efficiency indicates a large intrinsic non-radiative decay rate $\tau_{n r}$. 
As the quantum efficiency $Q E$ is a monotonic function of the Purcell factor $F_{P}$, when $\tau_{n r}$ is larger, or equivalently $Q E_{0}$ is smaller, the quantum efficiency tends to be more easily improved under the influence of the Purcell effect. The dependence of the quantum efficiency modification $Q E / Q E_{0}$ on the intrinsic quantum efficiency $Q E_{0}$ is shown in Figure 2.7, where it is assumed that the absorption-introduced non-radiative decay rate $\tau_{a b s}=0.1 \tau_{r a d}$ and $F_{P}=100$. This explains why a small quantum efficiency tends to be more easily improved.

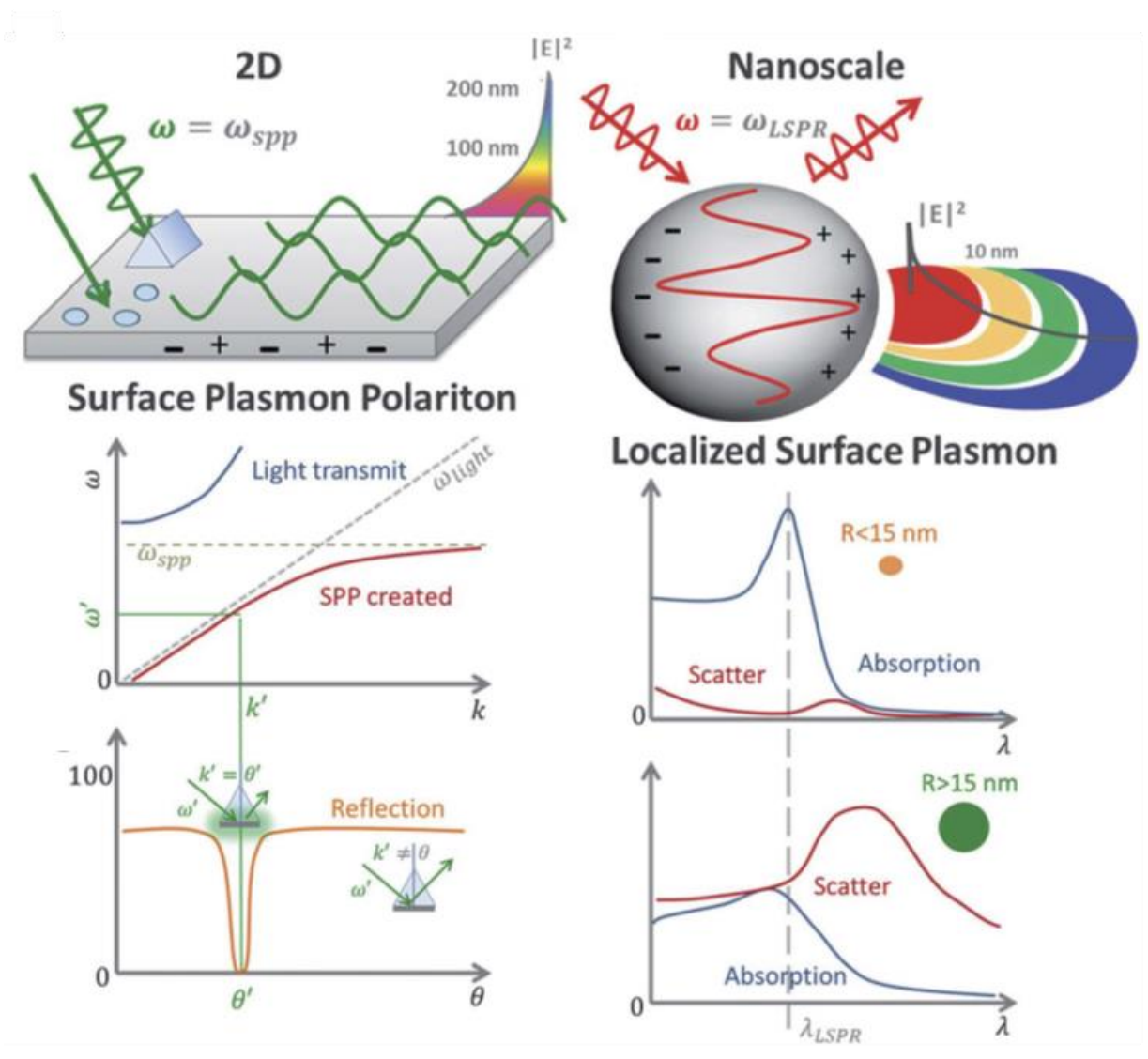

Figure 2.8 A comparison of surface plasmon polariton (SPP) and localized surface plasmon resonance (LSPR). ${ }^{7}$

\subsection{Surface Plasmon Resonance}

Surface plasmon, also called quasiparticle, is the direct result of the quantization of collective oscillation of conduction band electrons in solids. ${ }^{8}$ The plasma frequency of a metal $\omega_{p}=\sqrt{\frac{n_{e}^{2}}{m_{e f f} \epsilon_{0}}}$ is an intrinsic property of the material with $n_{e}$ being the electron density and $m_{e f f}$ being the effective mass. ${ }^{9}$ Surface plasmon resonance occurs in two different types: surface plasmon polariton (SPP) and localized surface plasmon resonance (LSPR). ${ }^{7}$ SPP is fundamentally a propagating electromagnetic plane wave trapped at the interface between metal and dielectric. Due to the 
intrinsic smaller wavevector of the free space light line compared to the dispersion relation of SPP in Figure 2.8, which indicates there is a lack a momentum conservation, SPP cannot be directly excited. Usually, a prism or grating is needed to supply the additional wavevector needed. With the development of nanofabrication, periodic nanostructures have also become increasingly used to excite SPP as the periodic structure acts as a grating. SPP decays up to $200 \mathrm{~nm}$ in the form of an evanescent field perpendicular to the metal surface and it can also radiatively mediate energy transfer. ${ }^{10-11}$ In contrast, LSPR can be directly excited on a subwavelength nanoparticle. Due to the excitation of LSPR, the gold nanoparticle shown in Figure 2.8 just acts as a lens, concentrating the incident light to a small volume on the surface of the nanoparticle, thus creating a very high near field which decays $\sim 10 \mathrm{~nm}$. With a small size $(<15 \mathrm{~nm})$, the nanoparticle absorbs light strongly and finally converts the light into heat due to electron-electron collisions. For a large size $(>15$ $\mathrm{nm}$ ), much of the absorbed light gets scattered to the far field. The interesting effect of the size on the transition from absorption-dominated to scattering-dominated extinction along with the strong near field has many implications in surface-enhanced spectroscopy. ${ }^{12-13}$

\subsection{Surface-Enhanced Raman Scattering}

SERS is an optical process where the chance of Raman scattering, a weak inelastic scattering, is drastically improved by the aid of surface plasmon resonance. ${ }^{12,14-15}$ Normal Raman scattering occurs approximately once in a million; SERS could improve that process by orders of magnitude. SERS enhancement is often attributed to electromagnetic (EM) enhancement and chemical enhancement. ${ }^{16-17}$ EM enhancement offers a SERS enhancement factor up to $10^{12}$; in comparison, chemical enhancement is only able to contribute a mild SERS enhancement in the order of $10^{2}$. Since EM enhancement is much larger than chemical enhancement, it has been exploited much more extensively in SERS applications. By a careful design of the nanostructure, a SERS enhancement factor of $10^{14}$ has been reported, which allows single molecule detection. ${ }^{18}$

There are many advantages with SERS. For instance, it can reveal molecular spectral fingerprint without any water interference; it is capable of multiplex detection with only minimal sample preparation; it is a high-throughput technique and can be used in point-of-care (POC) applications. ${ }^{12,14-15}$ If only considering EM enhancement, SERS enhancement is approximately proportional to the fourth power of local EM field enhancement, making it straightforward to engineer high-performance SERS substrates. Because of these advantages, SERS is one of the most popular techniques in optical sensing applications.

Conventional SERS sensors are based on colloidal Au nanoparticles. ${ }^{19}$ Since Au nanoparticles support localized surface plasmon resonance (LSPR), the absorbed Raman molecule would benefit from LSPR and produces SERS signals. However, colloidal nanoparticles are vulnerable to aggregation, they are not suitable to be directly implemented in building SERS sensors. To overcome colloidal nanoparticle aggregation, shell-isolated nanoparticle-enhanced Raman 
spectroscopy (SHINERS) is used, in which colloidal nanoparticles are protected by a thin silica shell. ${ }^{20}$ The SHINERS structure allows Raman molecules to be sandwiched between the silica shell and the nanoparticle, which end up as a SERS probe. This structure is water-soluble thanks to a hydrophilic silica surface, which further enables easy surface functionalization. ${ }^{21}$

The performance of SERS probes is dependent on the type of nanoparticles used. While Ag nanoparticles have an overall stronger plasmon strength than any other metallic nanoparticles, $\mathrm{Au}$ nanoparticles are most often used owing to the chemical stability. Strong LSPR is often found on $\mathrm{Au}$ nanoparticles with sharp features where surface charge density is high and can be easily polarized. ${ }^{22-23}$ Candidate Au nanostructures for SERS probes include Au nanorods, Au nanostars, and Au nanopyramid arrays, all of which support strong LSPR at their respective sharp regions.

Two-dimensional (2D) plasmonic nanoarrays are also exploited for SERS. ${ }^{24-25}$ In a $2 \mathrm{D}$ plasmonic nanoarray, not only LSPR, but also SPP can be excited, which significantly provides additional enhancement channels for SERS. ${ }^{26-27}$ 2D plasmonic nanoarrays can be directly used as a SERS sensing platform as well as be integrated with SERS probes. The biggest advantage of an integration is an enhanced performance because of plasmonic coupling between the SERS probe and the 2D plasmonic nanostructure, which could provide highly sensitive SERS sensing. ${ }^{28-29}$

Such a design has been implemented in biosensing in biological fluids. ${ }^{28-29}$ Take an immunoassay-based SERS biosensor as an example. The SERS probe and the 2D plasmonic nanoarray are initially functionalized with capture and detection antibodies. Upon the addition of the analyte which is the antigen, the immune-reaction would make the SERS probe captured on the 2D plasmonic nanoarray. After washing away excessive SERS probes which are not captured due to immuno-reactions, SERS signals can be detected from the 2D plasmonic nanoarray and scales with the concentration of the analyte in presence. If a near-infrared wavelength such as 785 $\mathrm{nm}$ is used as the excitation source and considering that the SERS signal is specific to the Raman molecule sandwiched in the SERS probe, minimal interference is expected from the surrounding environment. SERS sensors based on this mechanism have been demonstrated for detection of heavy metals and cancer biomarkers in human saliva and blood plasma.

Despite all these merits mentioned above, SERS unavoidably suffers from quite a few drawbacks, some of which are even fundamental to the technique itself. First, SERS sensors almost always require a SERS probe or a Raman molecule to transduce the SERS signal, because many of the analytes either are Raman inactive or display uncharacteristic Raman spectra. Second, the nature of scattering in SERS suggests that any scattering events from either the surrounding environment or interfering substances could blur the SERS signal by producing either specific interfering peaks or a strong background. This would significantly compromise the performance of SERS. Third, since intrinsic Raman scattering is too weak, it always needs to be amplified before any practical applications can happen. However, the SERS enhancement could vary enormously due to poor quality of colloidal nanoparticles or 2D plasmonic nanostructures. More 
importantly, high SERS spectral resolution requires a tightly focused beam, which significantly hinders the development of SERS-based POC sensor devices.

\subsection{Plasmon-Enhanced Fluorescence}

Similar to SERS, PEF is an optical process where the fluorescence emission is improved under the influence of surface plasmon resonance. ${ }^{4,} 30$ PEF can also reveal molecular spectral fingerprint without any water interference and achieves single molecule detection. More importantly, PEF has its own unique features.

First, the cross section of fluorescence is much larger than Raman scattering. ${ }^{31}$ A typical cross section for fluorescence is $\sim 10^{-20} \mathrm{~m}^{2}$, whereas that for non-resonant and resonant Raman is $\sim 10^{-33}$ $\mathrm{m}^{2}$ and $\sim 10^{-29} \mathrm{~m}^{2}$, respectively, which is a difference of 9 13 orders of magnitude! Therefore, even in the absence of plasmon enhancement, fluorescence is a highly competitive technique.

Second, fluorescence is robust with strong anti-interference capability. Fluorescence consists of a two-step process: absorption at a shorter wavelength and emission at a longer wavelength after a rapid relaxation process (Stokes shift). Although some substances may absorb or emit at the same wavelength as fluorescence, it is very rare for a substance to both absorb and emit at the same wavelengths as fluorescence. This unique feature makes fluorescence highly advantageous.

Third, fluorescence emission is not always improved under the influence of surface plasmon resonance; it can also be quenched. ${ }^{32}$ Fluorescence enhancement consists of excitation enhancement and emission enhancement. The excitation enhancement is the same for both SERS and PEF, which scales with the square of local EM field enhancement. However, the emission enhancement for fluorescence does not has a simple relationship with the local EM field enhancement. Instead, the probability for the excited carriers to radiatively decay depends on the available local density of optical states, which is determined by the Purcell factor. Moreover, the energy of the excited carriers could decay non-radiatively due to the absorption-induced energy loss by the plasmonic metallic nanostructures, which, if out-competes with the radiative counterpart, could lead to fluorescence quenching as well. Therefore, engineering PEF is a delicate task to balance the radiative and non-radiative decay.

Since most visible quantum dots and dyes are highly fluorescent with the quantum efficiency close to $100 \%$, they often do not need additional emission enhancement. Rather, they tend to be quenched when coupled with metallic nanostructures with the reason given in Section 2.1.6. But in the visible wavelength range, photobleaching could occur. Visible light could damage biological substances and induce addition background interference. Therefore, visible fluorescence is not ideal for sensing in biological samples. 
It is known that there are three biological transparency windows in the NIR wavelength range where there is minimal scattering and background fluorescence interference from biological matrix. ${ }^{33}$ The first biological window (NIR-I) spans from 700 to $950 \mathrm{~nm}$; the second (NIR-II) from 1000 to $1350 \mathrm{~nm}$; the third (NIR-III) from 1550 to $1870 \mathrm{~nm}$. They offer a great opportunity to employ NIR fluorescence for sensing in biological samples. However, NIR fluorophores are also known for a small intrinsic quantum efficiency. They are not suitable to be directly applied in NIR biosensing. Fortunately, PEF offers a solution.

The way plasmon mediates fluorescence is based tuning the distance between the fluorophore and the plasmonic nanostructure. The optimal distance varies for different plasmonic nanostructures, but in general, $10 \sim 20 \mathrm{~nm}$ is the typical range in which radiative decay dominates, contributing to overall fluorescence enhancement. Based on this, various nanostructures which can support strong surface plasmon resonance have been fabricated for this purpose. ${ }^{34-37}$

In a typical plasmon-enhanced fluorescence configuration, a gold nanorod as a plasmonic nanostructure is initially functionalized with biotin disulfide. ${ }^{38-39}$ Disulfide has a strong affinity towards gold surface and the biotin can interact with streptavidin. Therefore, streptavidin can be then functionalized to gold surface by biotin-streptavidin interaction. As the streptavidin has two binding sites, the other biotin which is at one end of a dye Cy5 labelled ssDNA sequence is then linked to gold nanrod by means of biotin-streptavidin. The ssDNA sets the dye a certain distance away from gold nanorod surface and by manipulating the number of DNA bases, as the length can be accurately tuned by simply changing the number of DNA bases. At an optimal distance, the plasmonic field contributes to the fluorescence enhancement of Cy5 by coupling the fluorophore dipole with surface plasmon dipole and then being scattered as fluorescence emission to the far field. The fluorescence imaging shows the fluorescence from $\mathrm{Cy} 5$ has been increased by around 30 times when linked to gold nanorod compared to free-standing Cy5. The dye also experiences a lifetime shortening, which can be ascribed to a faster radiative decay rate thanks to the modulation of surface plasmon on gold nanorod.

With gold nanorod as a typical gold nanoparticle which supports surface plasmon resonance, various two-dimensional plasmonic nanostructures have also been fabricated. Gold nanohole arrays, gold nanopyramid arrays, gold nanoring arrays, gold nanorod arrays, gold nanotriangle arrays, et al. are all capable of providing a strong plasmonic field, coupling with the fluorophore dipole.

\subsection{Rational of Selection of Nanoparticles/Nanostructures}

In what follows, design of SERS and PEF sensors as well as study of inexpensive alternative plasmonic material will be introduced. 
Chapter 3.1 is about design of a gold nanoparticle dimer-based SERS sensor for mercury detection; Chapter 3.2 is on design of an on-chip gold nanostar-coupled silver nanopyramid arraybased SERS sensor for nitrite detection. The reason why gold nanosphere is selected in Chapter 3.1 is because of its robust and well-studied surface chemistry. Although gold nanosphere is limited by the maximum achievable local fields, this issue can be overcome by the dimer structure where the plasmonic gap mode supports a large local field. In chapter 3.2, the selection of silver nanopyramid array is because 1-naphthylamine bonds to silver surface rather than gold surface. The selection of gold nanostar to couple with silver nanopyramid array is because gold nanostar supports strong local fields at the sharp tips, which is good for SERS.

Chapter 4.1 is on the elucidation of the role of scattering in PEF. The selection of silver nanosphere is because it is easy to tune its optical properties from being dominated by absorption to by scattering. This gives a unique window to study the role scattering plays in PEF. Chapter 4.2 is about a PEF sensor for traumatic brain injury biomarker detection in blood plasma. The rational for the selection of gold nanopyramid array is because it not only supports near-infrared plasmon resonance but also can generate strong local fields for excitation enhancement.

Chapter 5.1 is on the investigation of inexpensive alternative plasmonic metal. The reason why copper nanocube is selected is because copper is found to support a strong plasmon resonance only when the shape is made cubic. Chapter 5.2 is on the study of film-coupled copper nanocube for near-infrared fluorescence. This is an extension of Chapter 5.1 and strives to demonstrate that the inexpensive copper nanocube could be made spectrally tunable and useful in PEF. 


\section{Reference}

1. Zayats, A. V.; Smolyaninov, I. I.; Maradudin, A. A., Nano-optics of surface plasmon polaritons. Physics Reports 2005, 408 (3), 131-314.

2. Novotny, L.; Hecht, B., Principles of nano-optics. Cambridge University Press: Cambridge, 2006.

3. Haynes, C. L.; McFarland, A. D.; Van Duyne, R. P., Surface-Enhanced Raman Spectroscopy. Anal Chem 2005, 77 (17), 338 A-346 A.

4. Li, J.-F.; Li, C.-Y.; Aroca, R. F., Plasmon-enhanced fluorescence spectroscopy. Chem Soc Rev 2017, 46 (13), 3962-3979.

5. Cialla-May, D.; Zheng, X. S.; Weber, K.; Popp, J., Recent progress in surface-enhanced Raman spectroscopy for biological and biomedical applications: from cells to clinics. Chem Soc Rev 2017, 46 (13), 3945-3961.

6. Itoh, T.; Yamamoto, Y. S.; Ozaki, Y., Plasmon-enhanced spectroscopy of absorption and spontaneous emissions explained using cavity quantum optics. Chem Soc Rev 2017, 46 (13), 3904-3921.

7. Li, M.; Cushing, S. K.; Wu, N., Plasmon-enhanced optical sensors: a review. Analyst 2015, 140 (2), 386-406.

8. Anatoly, V. Z.; Igor, I. S., Near-field photonics: surface plasmon polaritons and localized surface plasmons. Journal of Optics A: Pure and Applied Optics 2003, 5 (4), S16.

9. $\quad$ Ordal, M. A.; Long, L. L.; Bell, R. J.; Bell, S. E.; Bell, R. R.; Alexander, R. W.; Ward, C. A., Optical properties of the metals Al, $\mathrm{Co}, \mathrm{Cu}, \mathrm{Au}, \mathrm{Fe}, \mathrm{Pb}, \mathrm{Ni}, \mathrm{Pd}, \mathrm{Pt}, \mathrm{Ag}, \mathrm{Ti}$, and $\mathrm{W}$ in the infrared and far infrared. Appl. Opt. 1983, 22 (7), 1099-1119.

10. Andrew, P.; Barnes, W. L., Energy Transfer Across a Metal Film Mediated by Surface Plasmon Polaritons. Science 2004, 306 (5698), 1002.

11. Collini, E.; Todescato, F.; Ferrante, C.; Bozio, R.; Scholes, G. D., Photophysics and Dynamics of Surface Plasmon Polaritons-Mediated Energy Transfer in the Presence of an Applied Electric Field. J Am Chem Soc 2012, 134 (24), 10061-10070.

12. Sharma, B.; Frontiera, R. R.; Henry, A.-I.; Ringe, E.; Van Duyne, R. P., SERS: Materials, applications, and the future. Materials Today 2012, 15 (1), 16-25.

13. Li, Z.; Wang, Y.; Wang, J.; Tang, Z.; Pounds, J. G.; Lin, Y., Rapid and Sensitive Detection of Protein Biomarker Using a Portable Fluorescence Biosensor Based on Quantum Dots and a Lateral Flow Test Strip. Anal Chem 2010, 82 (16), 7008-7014.

14. Sigle, D. O.; Kasera, S.; Herrmann, L. O.; Palma, A.; de Nijs, B.; Benz, F.; Mahajan, S.; Baumberg, J. J.; Scherman, O. A., Observing Single Molecules Complexing with Cucurbit uril through Nanogap Surface-Enhanced Raman Spectroscopy. The Journal of Physical Chemistry Letters 2016, 7 (4), 704-710.

15. Zrimsek, A. B.; Wong, N. L.; Van Duyne, R. P., Single Molecule Surface-Enhanced Raman Spectroscopy: A Critical Analysis of the Bianalyte versus Isotopologue Proof. The Journal of Physical Chemistry C 2016, 120 (9), 5133-5142. 
16. Stiles, P. L.; Dieringer, J. A.; Shah, N. C.; Van Duyne, R. P., Surface-Enhanced Raman Spectroscopy. Annu Rev Anal Chem 2008, 1 (1), 601-626.

17. Chen, L.; Li, Z.; Meng, Y.; Lu, M.; Wang, Z.; Zhang, R.-Q., Chemical Mechanism and Tunability of Surface-Enhanced Raman Scattering of Pyridine on Heteronuclear Coinage Metal Diatomic Clusters: A Density Functional Study. The Journal of Physical Chemistry C 2013, 117 (24), 12544-12551.

18. Liu, H.; Zhang, L.; Lang, X.; Yamaguchi, Y.; Iwasaki, H.; Inouye, Y.; Xue, Q.; Chen, M., Single molecule detection from a large-scale SERS-active Au79Ag21 substrate. Sci Rep-Uk 2011, 1,112 .

19. Leopold, N.; Chiş, V.; Mircescu, N. E.; Marişca, O. T.; Buja, O. M.; Leopold, L. F.; Socaciu, C.; Braicu, C.; Irimie, A.; Berindan-Neagoe, I., One step synthesis of SERS active colloidal gold nanoparticles by reduction with polyethylene glycol. Colloids and Surfaces A: Physicochemical and Engineering Aspects 2013, 436, 133-138.

20. Li, J. F.; Huang, Y. F.; Ding, Y.; Yang, Z. L.; Li, S. B.; Zhou, X. S.; Fan, F. R.; Zhang, W.; Zhou, Z. Y.; Wu, D. Y.; Ren, B.; Wang, Z. L.; Tian, Z. Q., Shell-isolated nanoparticle-enhanced Raman spectroscopy. Nature 2010, 464, 392.

21. Ming, L.; Scott, K. C.; Jianming, Z.; Jessica, L.; Zoraida, P. A.; Dongling, M.; Nianqiang, W., Shape-dependent surface-enhanced Raman scattering in gold-Raman-probe-silica sandwiched nanoparticles for biocompatible applications. Nanotechnology 2012, 23 (11), 115501.

22. Ferbonink, G. F.; Spada, E. R.; Santos, D. P. d.; Sartorelli, M. L.; Nome, R. A., Ultrafast Dynamics of Au Nanopyramid Interfaces Prepared by Nanosphere Lithography: Effect of Substrate Chemical Composition. J Brazil Chem Soc 2016, 27, 423-433.

23. Wang, P.; Xia, M.; Liang, O.; Sun, K.; Cipriano, A. F.; Schroeder, T.; Liu, H.; Xie, Y.-H., Label-Free SERS Selective Detection of Dopamine and Serotonin Using Graphene-Au Nanopyramid Heterostructure. Anal Chem 2015, 87 (20), 10255-10261.

24. Zheng, P.; Cushing, S. K.; Suri, S.; Wu, N., Tailoring plasmonic properties of gold nanohole arrays for surface-enhanced Raman scattering. Phys Chem Chem Phys 2015, 17 (33), 21211-21219.

25. Yabu, H., Bottom-Up Approach to Creating Three-Dimensional Nanoring Arrays Composed of Au Nanoparticles. Langmuir 2013, 29 (4), 1005-1009.

26. Rivas, J. G.; Vecchi, G.; Giannini, V., Surface plasmon polariton-mediated enhancement of the emission of dye molecules on metallic gratings. New J Phys 2008, 10 (10), 105007.

27. Kalachyova, Y.; Mares, D.; Lyutakov, O.; Kostejn, M.; Lapcak, L.; Švorčík, V., Surface Plasmon Polaritons on Silver Gratings for Optimal SERS Response. The Journal of Physical Chemistry C 2015, 119 (17), 9506-9512.

28. Zheng, P.; Li, M.; Jurevic, R.; Cushing, S. K.; Liu, Y.; Wu, N., A gold nanohole array based surface-enhanced Raman scattering biosensor for detection of silver(i) and mercury(ii) in human saliva. Nanoscale 2015, 7 (25), 11005-11012.

29. Li, M.; Cushing, S. K.; Zhang, J.; Suri, S.; Evans, R.; Petros, W. P.; Gibson, L. F.; Ma, D.; Liu, Y.; Wu, N., Three-Dimensional Hierarchical Plasmonic Nano-Architecture Enhanced 
Surface-Enhanced Raman Scattering Immunosensor for Cancer Biomarker Detection in Blood Plasma. Acs Nano 2013, 7 (6), 4967-4976.

30. Chu, L.-Q.; Förch, R.; Knoll, W., Surface-Plasmon-Enhanced Fluorescence Spectroscopy for DNA Detection Using Fluorescently Labeled PNA as "DNA Indicator". Angewandte Chemie International Edition 2007, 46 (26), 4944-4947.

31. Katrin, K.; Harald, K.; Irving, I.; Ramachandra, R. D.; Michael, S. F., Surface-enhanced Raman scattering and biophysics. Journal of Physics: Condensed Matter 2002, 14 (18), R597.

32. Anil Kumar, P.; Mohan, D. B., Fabrication of partially oxidized ultra-thin nanocrystalline silver films: effect of surface plasmon resonance on fluorescence quenching and surface enhanced Raman scattering. Mater Res Express 2014, 1 (2), 025014.

33. Hemmer, E.; Benayas, A.; Légaré, F.; Vetrone, F., Exploiting the biological windows: current perspectives on fluorescent bioprobes emitting above $1000 \mathrm{~nm}$. Nanoscale Horiz 2016, 1 (3), 168-184.

34. Yang, B.; Zhang, B.; Xu, Y.; Wang, W.; Shen, Y.; Zhang, A.; Xu, Z., Prospective study of early detection for primary liver cancer. $J$ Cancer Res Clin 1997, 123 (6), 357-360.

35. Menon, U., Ovarian cancer: challenges of early detection. Nat Clin Pract Oncol 2007, 4, 498.

36. Resch-Genger, U.; Grabolle, M.; Cavaliere-Jaricot, S.; Nitschke, R.; Nann, T., Quantum dots versus organic dyes as fluorescent labels. Nat Methods 2008, 5, 763.

37. Lakowicz, J. R.; Ray, K.; Chowdhury, M.; Szmacinski, H.; Fu, Y.; Zhang, J.; Nowaczyk, K., Plasmon-controlled fluorescence: a new paradigm in fluorescence spectroscopy. Analyst 2008, 133 (10), 1308-1346.

38. Damm, S.; Fedele, S.; Murphy, A.; Holsgrove, K.; Arredondo, M.; Pollard, R.; Barry, J. N.; Dowling, D. P.; Rice, J. H., Plasmon enhanced fluorescence studies from aligned gold nanorod arrays modified with SiO2 spacer layers. Appl Phys Lett 2015, 106 (18), 183109. 39. Fu, Y.; Zhang, J.; Lakowicz, J. R., Plasmon-Enhanced Fluorescence from Single Fluorophores End-Linked to Gold Nanorods. J Am Chem Soc 2010, 132 (16), 5540-5541. 


\section{Chapter 3: Plasmonic Nanostructures for Surface- Enhanced Raman Scattering Sensing}

\subsection{A SERS Sensor for Mercury (II) Detection}

\subsubsection{Introduction}

Heavy metal pollution imposes a threat on human health. ${ }^{1-2} \mathrm{Hg}^{2+}$ is one of the most toxic cations, which causes damage to the central nervous system and other organs. ${ }^{3-4}$ Significant effort has been made to develop sensors for detection of $\mathrm{Hg}^{2+}$ ions. One of the most appealing sensors is based on colorimetric transduction. ${ }^{5-8}$ Gold nanoparticles (NPs) in an aqueous solution display a range of colors from red to blue due to the excitation of localized surface plasmon resonance (LSPR). The addition of target cations can induce the aggregation of Au NPs modified with small molecules, leading to a change in the color of solution. The colorimetric sensor is simple, straightforward, and requires minimal instrumentation. However, it has a low sensitivity, and aggregation of $\mathrm{Au}$ NPs can lead to sediment of Au NPs on the bottom of aqueous solution, limiting the dynamic detection range of assay.

Surface-enhanced Raman scattering (SERS) has emerged as a powerful technique in building sensitive sensors. ${ }^{9-13}$ The SERS signal can be amplified using plasmonic nanostructures, including colloidal NPs and the patterned nanostructures via either electromagnetic enhancement, chemical enhancement, or a combination of both. ${ }^{12,14-15}$ Recently SERS biosensors have been exploited for $\mathrm{Hg}^{2+}$ detection based on the formation of gold nanostar dimers or a hierarchical structure of gold nanostars on a gold nanohole array. ${ }^{10,16-17}$ In these SERS sensors, a thymine nucleobase (T) has been used to capture the $\mathrm{Hg}^{2+}$ ions by forming the $\mathrm{T}-\mathrm{Hg}$-T structure; and a Raman label has been used to track the SERS signal.

Herein, we report a SERS biosensor using a small molecule as the molecular recognition probe. The molecular probe enables a very simple but highly sensitive and selective approach for the detection of $\mathrm{Hg}^{2+}$ based on a newly emerging peak.

\subsubsection{Methods}

\section{Chemicals and Materials}

Chloroauric acid trihydrate $\left(\mathrm{HAuCl}_{4} \cdot 3 \mathrm{H}_{2} \mathrm{O}\right)$, trisodium citrate dihydrate $\left(\mathrm{Na}_{3} \mathrm{C}_{6} \mathrm{H}_{5} \mathrm{O}_{7} \cdot 2 \mathrm{H}_{2} \mathrm{O}\right.$, 
ACS, 90.0+\%), sodium hydroxide, silver nitrate $\left(\mathrm{AgNO}_{3}\right.$, Premion, 99.995\%), calcium nitrate tetrahydrate $\left(\mathrm{Ca}\left(\mathrm{NO}_{3}\right)_{2} \cdot 4 \mathrm{H}_{2} \mathrm{O}, 99.0 \%\right)$, iron(III) nitrate nonahydrate $\left(\mathrm{Fe}\left(\mathrm{NO}_{3}\right)_{3} \cdot 9 \mathrm{H}_{2} \mathrm{O}, 98+\%\right)$, copper(II) nitrate hemi(pentahydrate) $\left(\mathrm{Cu}\left(\mathrm{NO}_{3}\right)_{2} \cdot 2.5 \mathrm{H}_{2} \mathrm{O}\right)$, and yttrium(III) nitrate hexahydrate $\left(\mathrm{Y}\left(\mathrm{NO}_{3}\right)_{3} \cdot 6 \mathrm{H}_{2} \mathrm{O}, 99.9 \%\right.$ ) were purchased from Alfa Aesar (Ward Hill, MA). 4-mercaptobenzoic acid (technical grade, 90\%), 2,6-pyridinedicarboxylic acid (99\%), mercury nitrate $\left(\left(\mathrm{HgNO}_{3}\right)_{2} \cdot 2 \mathrm{H}_{2} \mathrm{O}\right)$, and chromium(III) nitrate nonahydrate $\left(\mathrm{Cr}\left(\mathrm{NO}_{3}\right)_{3} \cdot 9 \mathrm{H}_{2} \mathrm{O}\right)$ were purchased from Sigma-Aldrich (St Louis, MO). Methylmercury (II) chloride (standard solution in $\mathrm{H}_{2} \mathrm{O}$ was purchased from VWR International. Aluminium nitrate nonahydrate $\left(\mathrm{Al}\left(\mathrm{NO}_{3}\right)_{3} \cdot 9 \mathrm{H}_{2} \mathrm{O}, 99+\%\right)$ was from Acros Organics (Fair Lawn, NJ). Zinc nitrate hexahydrate $\left(\mathrm{Zn}\left(\mathrm{NO}_{3}\right)_{2} \cdot 6 \mathrm{H}_{2} \mathrm{O}, 98 \%\right)$ was from Strem Chemicals (Newburyport, NA). Deionized (D.I.) water was produced by using a Milli-Q Millipore system (18.2 M $\Omega \mathrm{cm}$, Millipore Corp., Billerica, MA).

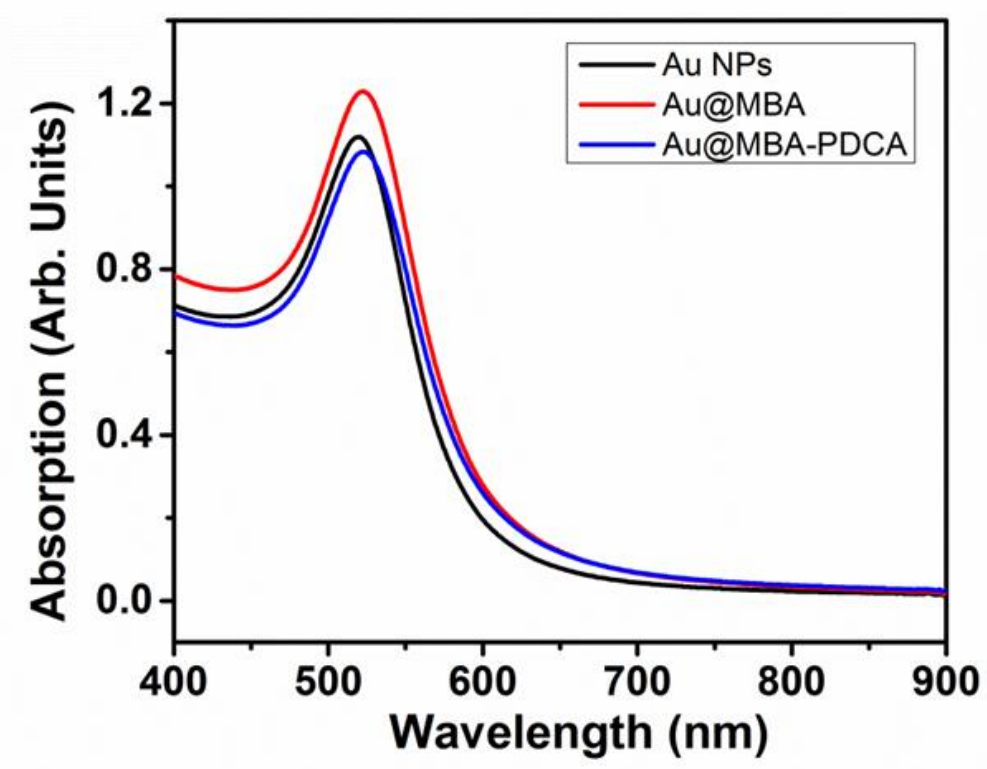

Figure 3.1 UV-Visible spectra of the as-prepared gold nanoparticles, Au@MBA solution, and a mixed solution of Au@MBA and PDCA.

\section{Preparation of Gold Nanoparticles}

$1.0 \mathrm{~mL}$ of $20.0 \mathrm{mM}$ chloroauric acid was added to $50.0 \mathrm{~mL}$ of deionized (D.I.) water and heated to boiling. Then, $2.0 \mathrm{~mL}$ of $38.8 \mathrm{mM}$ trisodium citrate solution was added. The reaction lasted 30 min under stirring. The absorption spectrum of synthesized gold nanoparticles was characterized using a Shimadzu UV-2550 spectrometer (Figure 3.1).

$10.0 \mu \mathrm{L}$ of $10.0 \mathrm{mM}$ freshly prepared 4-mercaptobenzoic acid (MBA) was added to $10.0 \mathrm{~mL}$ of as-prepared gold nanoparticles; and it was kept stirring overnight. Then Au@MBA nanoparticles were obtained without any purification, as the amount of MBA is far from excessive in order to keep gold nanoparticles from aggregation. The absorption peak of Au@MBA barely 
shifted compared to that of the as-synthesized gold nanoparticles, as shown in Figure 3.1.

\section{$\mathrm{Hg}^{2+}$ Detection}

The Raman spectroscopy (iRaman plus, Model\# BWS465, B\&W Tek) with an excitation wavelength of $785 \mathrm{~nm}$ was used to take all Raman spectra.

Before $\mathrm{Hg}^{2+}$ detection, $2 \mu \mathrm{L}$ of $10 \mathrm{mM}$ masking reagent 2,6-pyridinedicarboxylic acid (PDCA) was spiked with $10 \mu \mathrm{L}$ of $\mathrm{Au} @ \mathrm{MBA}$ nanoparticles at $\mathrm{pH}$ 7. The addition of PDCA did not induce the aggregation of gold nanoparticles, as confirmed by the UV-Vis spectrum in Figure 5.1. Then 2 $\mu \mathrm{L}$ of different concentrations of $\mathrm{Hg}^{2+}$ from $10 \mathrm{ppt}$ to $500 \mathrm{ppb}$ was added to the above solution and left it incubation overnight before Raman characterizations.

During Raman characterization, $0.5 \mu \mathrm{L}$ of the above solution was dropped on the gold film substrate (purchased from Amsbio, Catalogue No. AU.1000.ALSI). A $785 \mathrm{~nm}$ laser was used to illuminate it while a Timeline Acquisition was used to obtain the SERS spectra in a time interval of 1 second with an integration time of 1 second as well. At the beginning, the SERS spectra were barely seen. As the water solvent evaporated under laser illumination, which left gold nanoparticles increasingly concentrated, the SERS spectra of MBA gradually showed up. Seconds before the solution became dried, a new peak at $374 \mathrm{~cm}^{-1}$ appeared. This peak was attributed to a coordinate bond involving $\mathrm{Hg}^{2+}$, shown in Figure 3.2(d). 


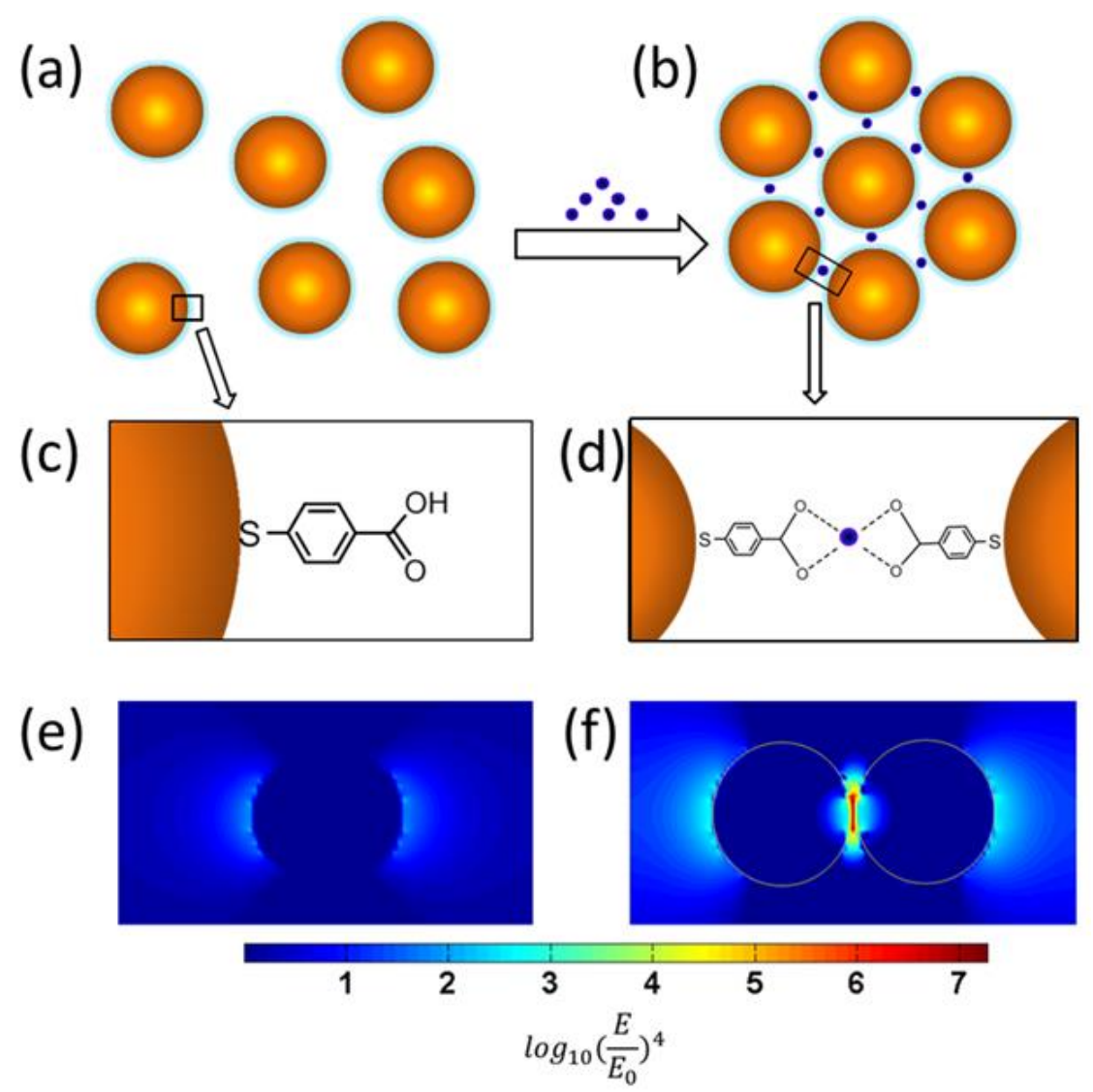

\section{Gold nanoparticles Metal ions}

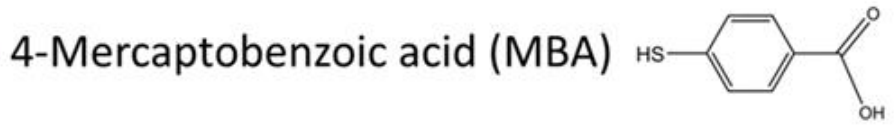

Figure 3.2 (a) Gold NPs functionalized with MBA; (b) coupling of Au NPs in the presence of metal ions; the zoom-in area and the corresponding electric field distribution shown in (c) and (e) for free-standing gold NPs and in (d) and (f) for coupled ones.

\section{Finite Difference Time Domain Simulations}

Optiwave software was used to conduct simulation. The simulation cell was constructed with a $1 \mathrm{~nm}$ of grid size. A plane wave of $785 \mathrm{~nm}$ was used as the input source.

\section{Principal Component Analysis}

Principal component analysis (PCA) was performed on the SERS spectra to demonstrate the improved selectivity achieved by the addition of PDCA into the sensing assay. PCA is an orthogonal linear transformation that transforms the data to a new coordinate system, in which the first coordinate (i.e., the first principal component) represents the direction of the greatest variability, and the second coordinate the direction of the second greatest variability, and so on. 


\subsubsection{Results and Discussions}

In this work, a SERS sensor is developed for $\mathrm{Hg}^{2+}$ detection using the gold NPs functionalized with 4-mercaptobenzoic acid (MBA), noted as Au@MBA, as shown in Figure 3.2(a) and (c). The carboxylic group enables the Au@MBA to be water soluble. When metal ions are absent in the aqueous solution, the Au@MBA NPs are kept distant from each other due to repulsion. The SERS peak intensity of MBA is relatively weak (Figure 3.3).

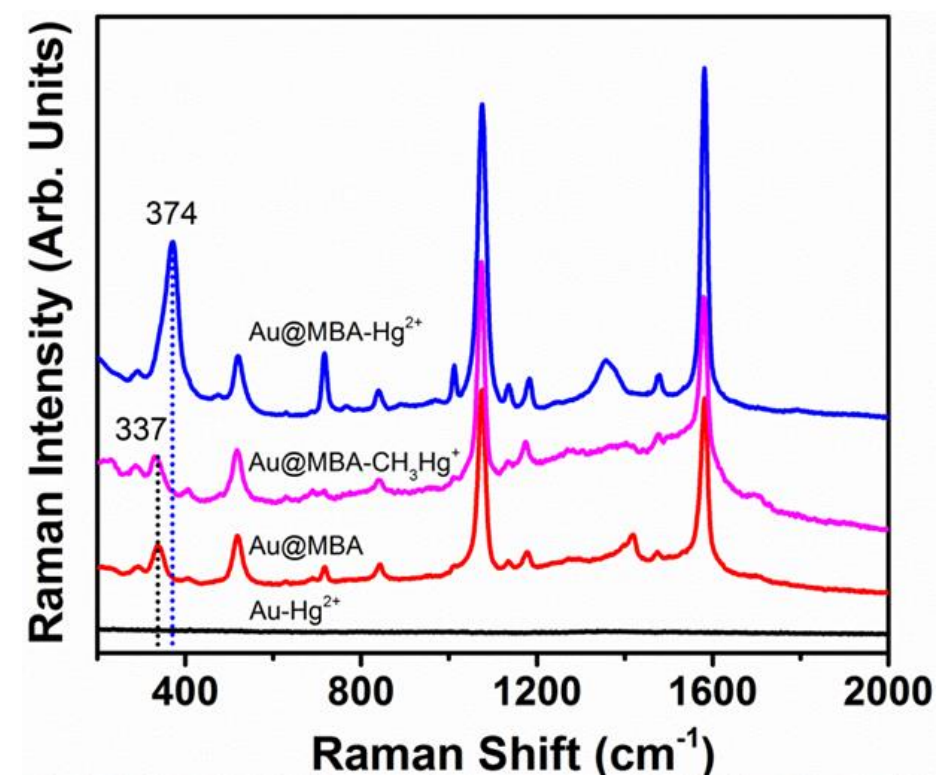

Figure 3.3 SERS spectra comparison. A mixture of $\mathrm{Au}-\mathrm{Hg}^{2+}$ without MBA does not generate SERS peaks; Au@MBA exhibits the characteristic peaks of MBA; the Au@MBA- $\mathrm{Hg}^{2+}$ complex generates a fingerprint peak at $374 \mathrm{~cm}^{-1}$. Other major peak assignments: $v(C O O-)\left(337 \mathrm{~cm}^{-1}, 1580 \mathrm{~cm}^{-1}\right), \mathrm{v}(\mathrm{CC})(1074$ $\left.\mathrm{cm}^{-1}, 1580 \mathrm{~cm}^{-1}\right), \delta(\mathrm{COO}-)\left(840 \mathrm{~cm}^{-1}\right), \delta(\mathrm{CH})\left(1136, \mathrm{~cm}^{-1}, 1183 \mathrm{~cm}^{-1}\right), \mathrm{Y}(\mathrm{CC})\left(520 \mathrm{~cm}^{-1}, 718 \mathrm{~cm}^{-1}\right)$.

When metal ions are present, the carboxylic group captures the metal ions due to the coordinate bond formed with $\mathrm{Hg}^{2+}$, leading to gold NP dimers, trimers and et al., as shown in Figure 3.2(b) and (d). The small gap between the adjacent Au@MBA NPs creates strong "hot spot", amplifying the electromagnetic field remarkably, as shown in Figure 3.2(e) and (f). Consequently, the SERS peak intensity of MBA increases considerably (Figure 3.3). More importantly, a fingerprint SERS peak appears at $374 \mathrm{~cm}^{-1}$ due to the binding of $\mathrm{Hg}^{2+}$ to the Au@MBA NPs (Figure 3.3). It is worth noting that the peak at $337 \mathrm{~cm}^{-1}$ disappeared after the $\mathrm{Hg}^{2+}$ ions are captured between the Au@MBA NPs (Figure 3.3). In addition, this phenomenon does not happen in the presence of $\mathrm{CH}_{3} \mathrm{Hg}^{+}$. Moreover, no evident SERS signal can be detected when $\mathrm{Hg}^{2+}$ is present in the non-functionalized Au NP suspension, ruling out the possible interference from chemicals used. Therefore, the $374 \mathrm{~cm}^{-1}$ peak comes from a Raman vibration of the newly formed coordinate bond involving $\mathrm{Hg}^{2+}$, shown in Figure 3.2(d). 
Although previous SERS sensors have been developed for $\mathrm{Hg}^{2+}$ detection, a Raman label has been used besides the use of a molecular recognition probe. In contrast, for the present SERS assay, the binding of $\mathrm{Hg}^{2+}$ with the molecular recognition probe, MBA, generates a fingerprint SERS peak, which makes it unnecessary to use a Raman label.

The selectivity of $\mathrm{Au} @$ MBA-based SERS assay toward $\mathrm{Hg}^{2+}$ was tested and tuned. When the other metal ions $(\mathrm{M})$ were present in the Au@MBA-based SERS assay, where $\mathrm{M}$ stands for $\mathrm{Al}^{3+}$, $\mathrm{Cr}^{2+}, \mathrm{Cu}^{2+}, \mathrm{Fe}^{3+}, \mathrm{Y}^{3+}, \mathrm{Zn}^{2+}, \mathrm{Ca}^{2+}, \mathrm{Pb}^{2+}, \mathrm{As}^{2+}, \mathrm{Cd}^{2+}$, respectively, the SERS spectra were acquired from the Au@MBA-based SERS assay one by one (Figure 3.4). The presence of any of three metal ions $\left(\mathrm{Pb}^{2+}, \mathrm{As}^{2+}\right.$ and $\left.\mathrm{Cd}^{2+}\right)$ induced a SERS peak at $374 \mathrm{~cm}^{-1}$. In order to overcome this crossselectivity problem, a reagent of 2,6-pyridinedicarboxylic acid (PDCA) was used as a mask agent. ${ }^{5}$, 11, 18 When $\mathrm{Hg}^{2+}$ co-exists with other metal ions such as $\mathrm{Pb}^{2+}, \mathrm{As}^{2+}$ and $\mathrm{Cd}^{2+}$ in the absence of PDCA (see Case 1 in Figure 3.5), these metal ions could be captured by MBA, leading to coupling of gold NPs. Consequently, any among $\mathrm{Hg}^{2+}, \mathrm{Pb}^{2+}, \mathrm{As}^{2+}$ or $\mathrm{Cd}^{2+}$ contributes to the new SERS peak at $374 \mathrm{~cm}^{-1}$, as shown in Figure 3.4(b). When PDCA is present but in the absence of $\mathrm{Hg}^{2+}$ (see Case 2 in Figure 3.5), the free-standing PDCA molecules capture all the metal ions because PDCA has higher bonding affinity toward other metal ions than $\mathrm{Hg}^{2+}$. Consequently, the Au@MBA NPs keep distant from each other. When $\mathrm{Hg}^{2+}$ co-exists with other metal ions in the presence of PDCA (see Case 3 in Figure 3.5), all the other metal ions are captured by PDCA. The free $\mathrm{Hg}^{2+}$ ions are captured by MBA, leading to coupling of Au@MBA NPs, generating the SERS peak at $374 \mathrm{~cm}^{-1}$, as shown in Figure 3.4(a). Hence the addition of PDCA enables the selective detection of $\mathrm{Hg}^{2+}$.
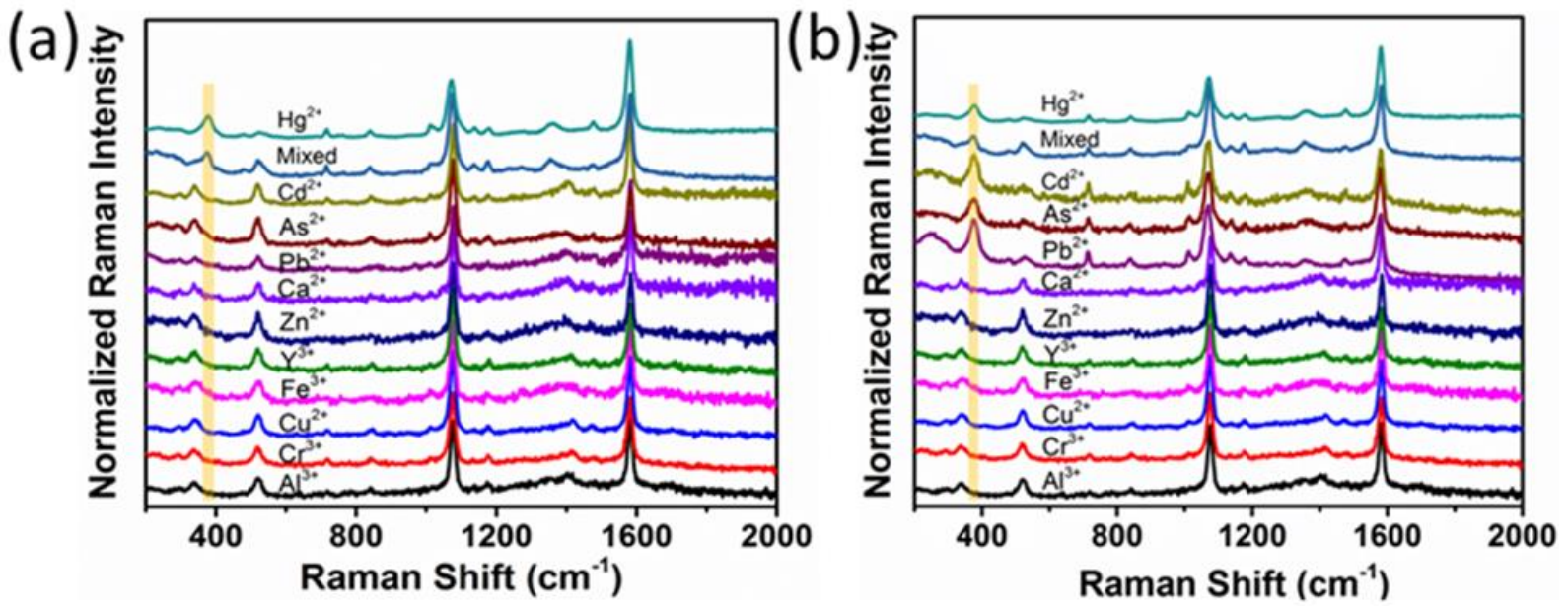

Figure 3.4 Effect of PDCA addition on the selectivity of sensing assay. (a) SERS spectra obtained from the sensing assay in the presence of PDCA when individual $\mathrm{M}$ ions (where $\mathrm{M}=\mathrm{Hg}^{2+}, \mathrm{Al}^{3+}, \mathrm{Cr}^{2+}, \mathrm{Cu}^{2+}$, $\mathrm{Fe}^{3+}, \mathrm{Y}^{3+}, \mathrm{Zn}^{2+}, \mathrm{Ca}^{2+}, \mathrm{Pb}^{2+}, \mathrm{As}^{2+}, \mathrm{Cd}^{2+}$ ) or a mixture of the above metal ions is present in the sensing assay; (b) SERS spectra obtained from the sensing assay in the absence of PDCA when individual $M$ ions (where $\mathrm{M}=\mathrm{Hg}^{2+}, \mathrm{Al}^{3+}, \mathrm{Cr}^{2+}, \mathrm{Cu}^{2+}, \mathrm{Fe}^{3+}, \mathrm{Y}^{3+}, \mathrm{Zn}^{2+}, \mathrm{Ca}^{2+}, \mathrm{Pb}^{2+}, \mathrm{As}^{2+}, \mathrm{Cd}^{2+}$ ) or a mixture of the above metal ions is present in the sensing assay. 
Case 1 Co-existence of $\mathrm{Hg}^{2+}$ and other metal ions in the absence of PDCA
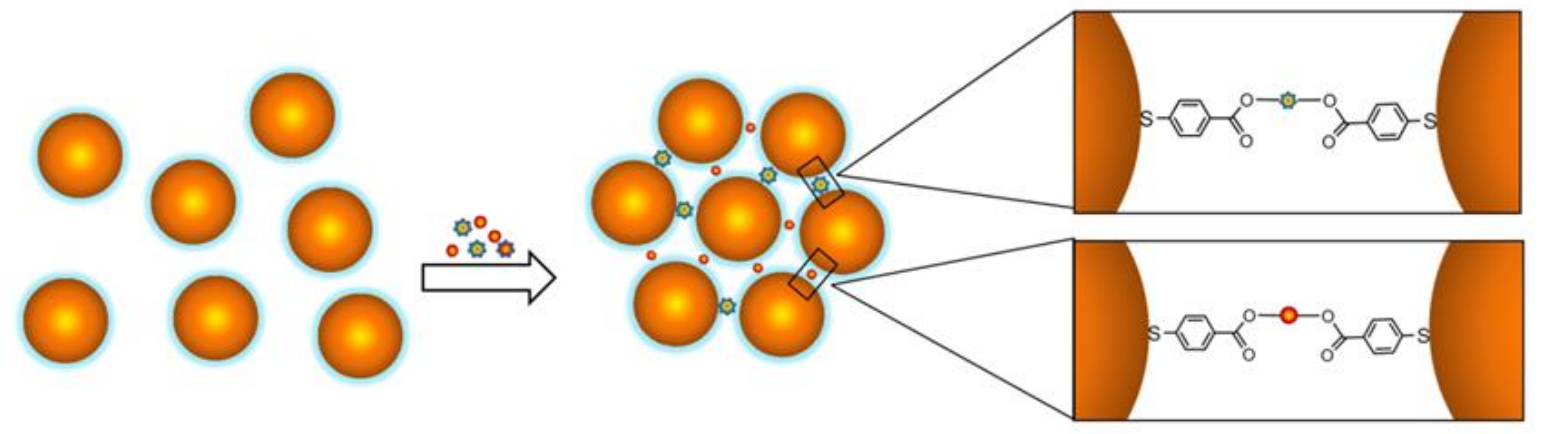

Case 2 Metal ions not including $\mathrm{Hg}^{2+}$ in the presence of PDCA
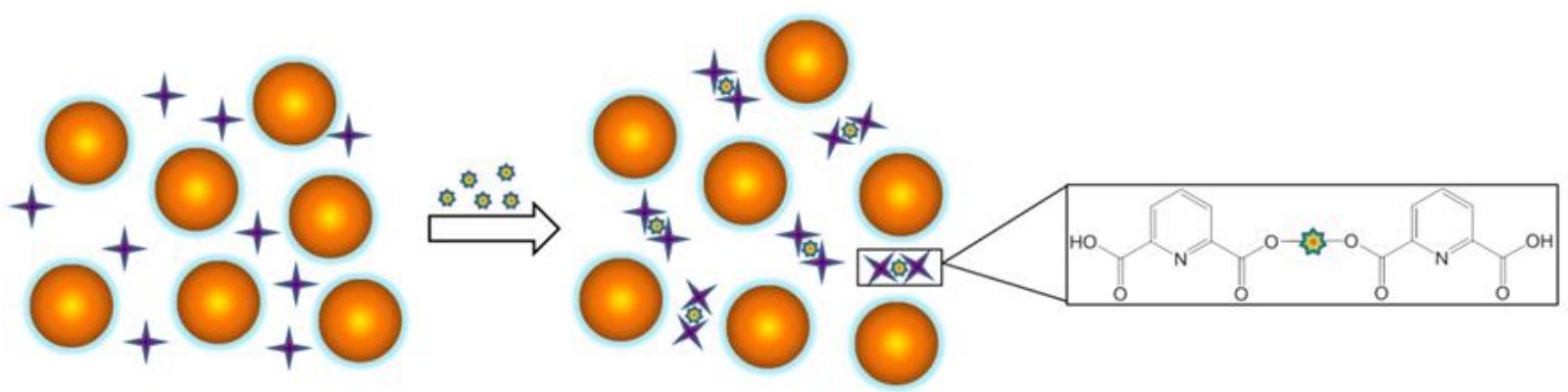

Case 3 Co-existence of $\mathrm{Hg}^{2+}$ and other metal ions in the presence of PDCA
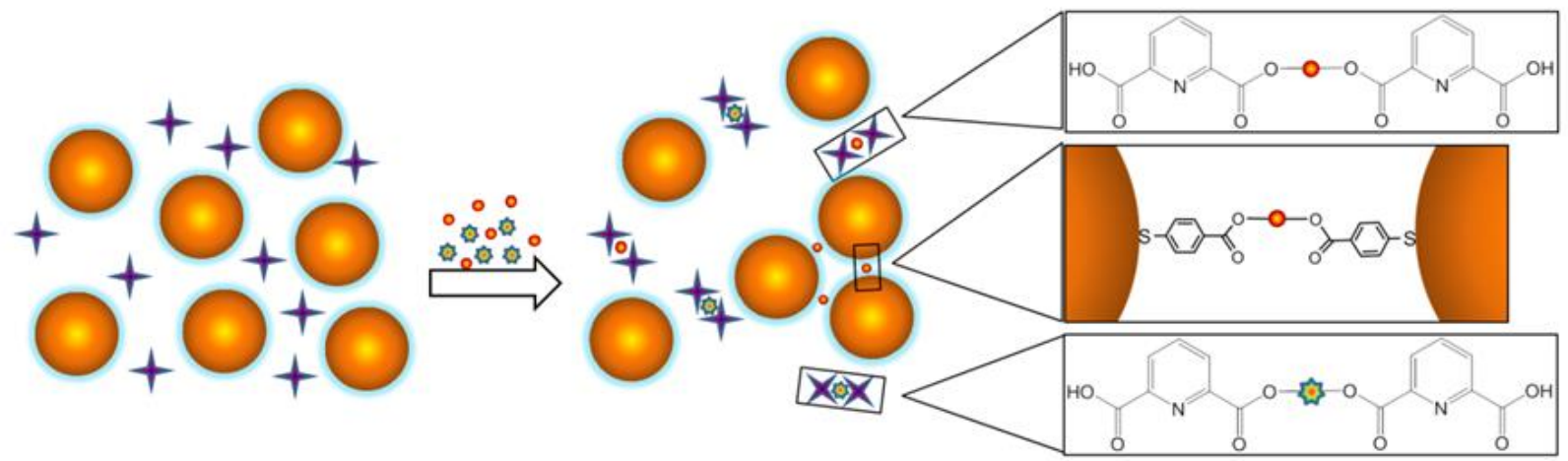

Gold nanoparticles

- $\mathrm{Hg}^{2+}$

Metal ions other than $\mathrm{Hg}^{2+}$

4-Mercaptobenzoic acid (MBA)

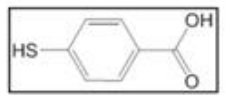

+ 2,6-pyridinedicarboxylic acid (PDCA)

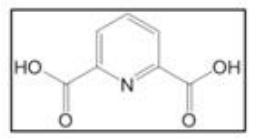

Figure 3.5 Schematic mechanism of PDCA in masking other metal ions 
Principal component analysis (PCA) was performed to quantify the effect of the PDCA addition on the selectivity of Au@MBA-based SERS assay. PCA was first carried out on the SERS spectra obtained from the sensing assay in the absence of PDCA when individual $\mathrm{M}$ ions (where $\mathrm{M}=\mathrm{Hg}^{2+}, \mathrm{Al}^{3+}, \mathrm{Cr}^{2+}, \mathrm{Cu}^{2+}, \mathrm{Fe}^{3+}, \mathrm{Y}^{3+}, \mathrm{Zn}^{2+}, \mathrm{Ca}^{2+}, \mathrm{Pb}^{2+}, \mathrm{As}^{2+}, \mathrm{Cd}^{2+}$ ) or a mixture of the above metal ions is present in the sensing assay (Figures 3.4(b) and 3.6).

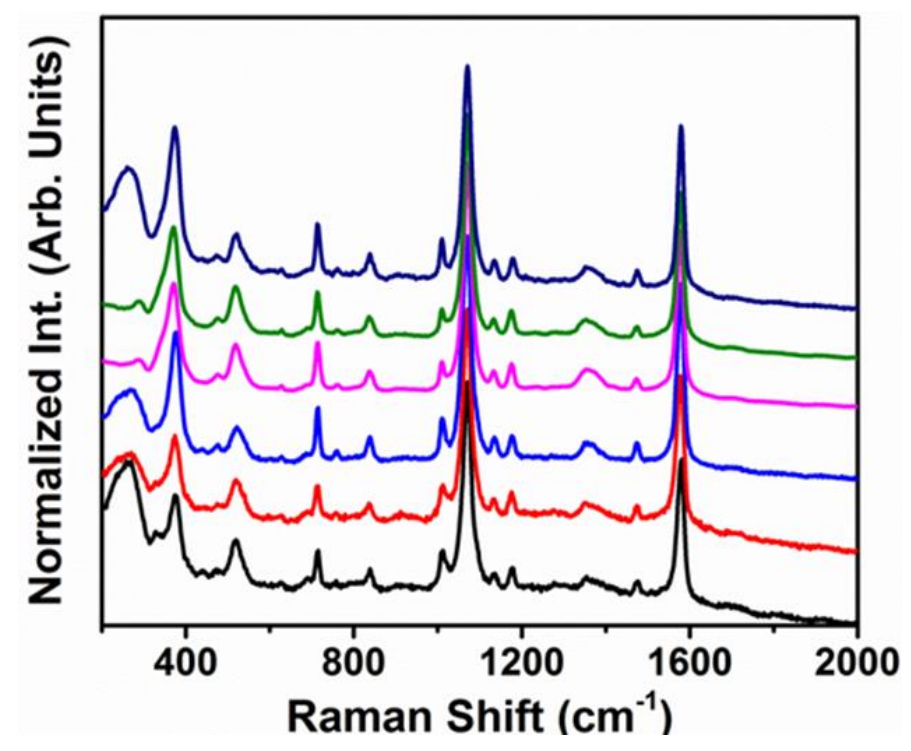

Figure 3.6 SERS spectra obtained from the sensing assay at different levels of $\mathrm{Hg}^{2+}$ ions in the absence of PDCA. The concentrations of $\mathrm{Hg}^{2+}$ from the bottom spectrum to the top one are: $50 \mathrm{ppt}, 100 \mathrm{ppt}, 500$ ppt, $1 \mathrm{ppb}, 10 \mathrm{ppb}$, and $50 \mathrm{ppb}$, respectively.

The PCA results are shown in Figure 3.7(a) that illustrates the two-dimensional coordinate plane with the axes being the first two principal components; and each point in the graph represents the projection of a spectrum onto the two-dimensional space, which corresponds to a specific cation of a certain concentration. Each spectrum is projected as a point in the resulting principal component space, and the confidence ellipse is generated for the $\mathrm{Hg}^{2+}$ points. The ellipse is the $95 \%$ confidence region estimated from the all the $\mathrm{Hg}^{2+}$ points. It can be seen from Figure 3.7(a) that the points of $\mathrm{Hg}^{2+}$ with varying concentrations are mixed with the points of other metal ions, which indicates that the sensing assay without PDCA exhibits poor selectivity toward $\mathrm{Hg}^{2+}$ detection. In contrast, Figure 3.7(b) provides the counterpart PCA results obtained from the SERS spectra of the sensing assay with PDCA (Figures 3.4(a) and 8). It clearly shows that a cluster of $\mathrm{Hg}^{2+}$ points with different concentrations in the ellipse are separated completely from the other metal ions. Thus, the PCA results have further confirmed the role of PDCA in improving the selectivity. 

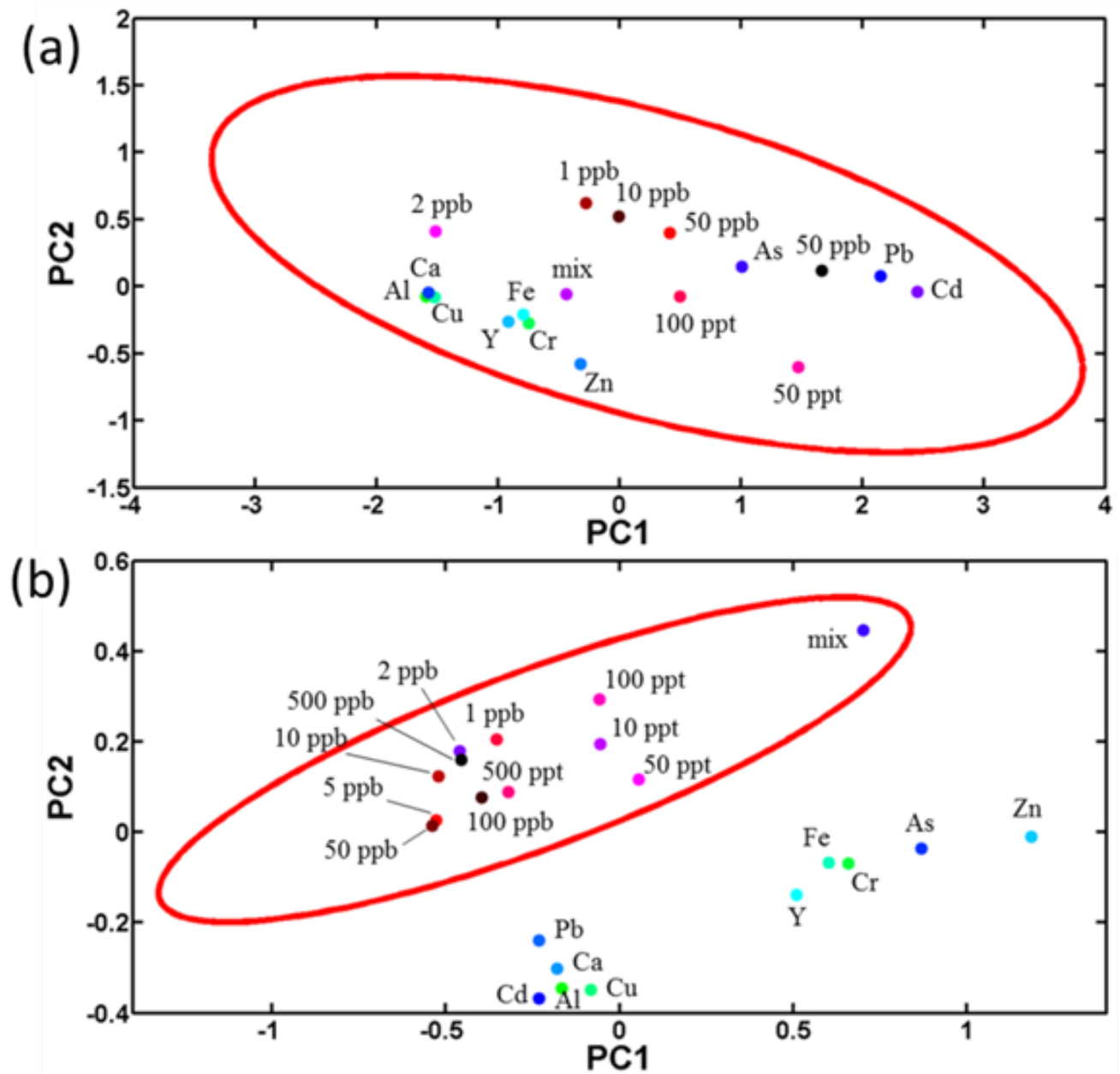

Figure 3.7 Principal component analysis of the SERS spectra obtained from the sensing assay in the absence of PDCA (a), and in the presence PDCA (b). 

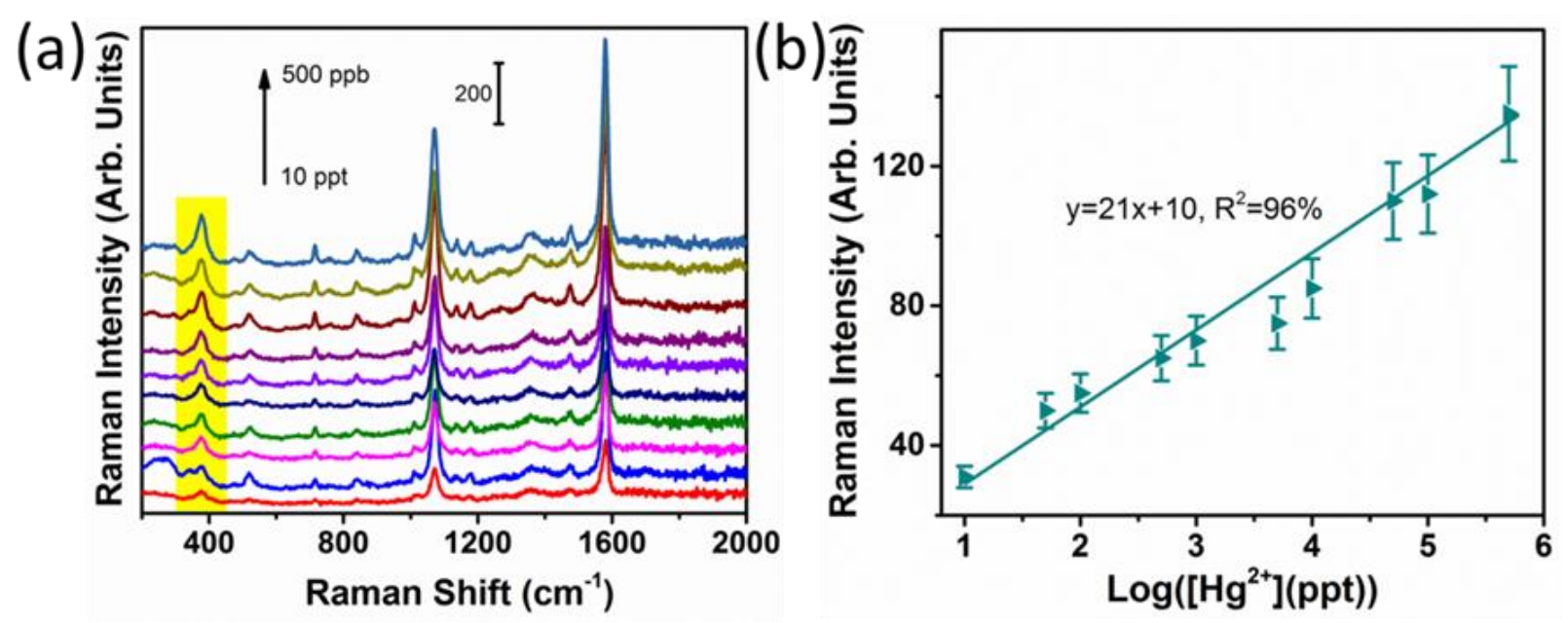

Figure 3.8 (a) SERS spectra obtained from the sensing assay in the presence of PDCA at different concentrations of $\mathrm{Hg}^{2+}(10 \mathrm{ppt}, 50 \mathrm{ppt}, 100 \mathrm{ppt}, 500$ ppt, 1 ppb, 5 ppb, 10 ppb, 50 ppb, 100 ppb, and 500 $\mathrm{ppb}$ ); (b) Calibration curve showing the SERS peak intensity at $374 \mathrm{~cm}^{-1}$ versus the logarithmic concentration of $\mathrm{Hg}^{2+}$.

The Au@MBA-based SERS assay was used to quantitatively measure the $\mathrm{Hg}^{2+}$ concentration in the presence of $10 \mathrm{mM}$ PDCA at pH 7 (Figure 3.8(a)). The intensity of SERS peak at $374 \mathrm{~cm}^{-1}$ increased with an increase of the $\mathrm{Hg}^{2+}$ concentration in a range of $10 \mathrm{ppt}$ up to $500 \mathrm{ppb}$. The peak intensity was linearly correlated to the logarithmic concentration of $\mathrm{Hg}^{2+}$ with a correlation coefficient of $\mathrm{R}^{2}=0.96$ (Figure 3.8(b)). The limit of detection (LOD) was estimated to be about 5 ppt based on the definition of $3 \delta / \mathrm{s}$, where $\delta$ is the standard deviation and $\mathrm{s}$ is the slope. ${ }^{19}$ Such high sensitivity can be attributed to the "hot spots" generated at the gaps between any adjacent coupled gold NPs in the presence of $\mathrm{Hg}^{2+}$, as shown in Figure 3.2(f). Finite-difference time domain (FDTD) simulation was conducted to confirm the high electromagnetic field generated at the gap between two coupled gold NPs, which shows that the maximum electromagnetic field enhancement factor $\left(\mathrm{E} / \mathrm{E}_{0}\right)^{4}$ can be as high as $10^{7}$ at a $1 \mathrm{~nm}$ gap, as shown in Figure 3.9. This was why the SERS signal can still be detected when the $\mathrm{Hg}^{2+}$ was down to the ppt level.

It is worth noting that the stability of plasmonic gold nanoparticles is dependent on the ionic strength of an aqueous solution where gold nanoparticles stay. Gold nanoparticles are instable when they are present in the aqueous solution with high ionic strength. In contrast, gold nanoparticles are stable when they are in the aqueous solution with low ionic strength. In this project, the SERS sensor based on the gold nanoparticles will be applied to river and lake water as well as tap water. However, the SERS sensor cannot be applied to the aqueous solution with high ionic strength, such as blood. 


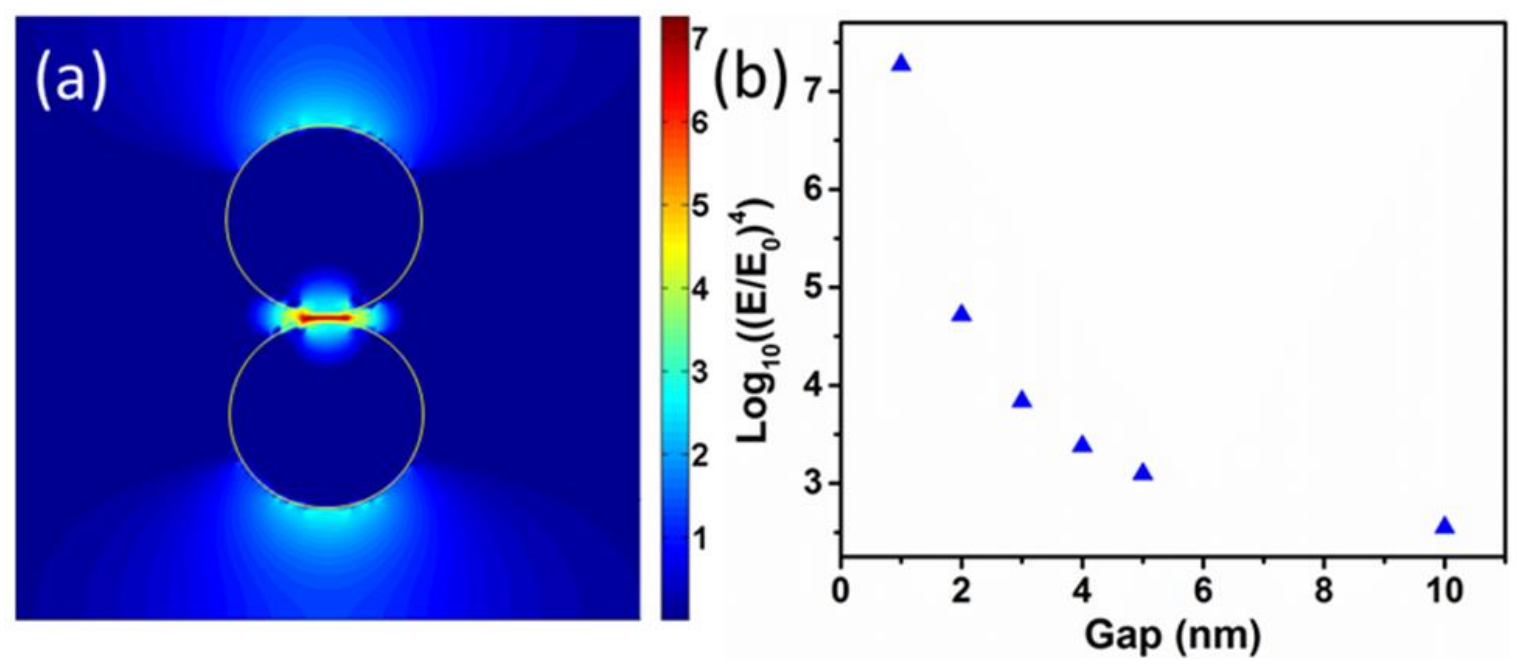

Figure 3.9 FDTD simulations (a) The electric field distribution at a $1 \mathrm{~nm}$ gap between two coupled gold nanoparticles. The SERS enhancement factor $\left(E / E_{0}\right)^{4}$ is $\sim 10^{7}$; (b) Gap-dependent SERS enhancement factor.

\subsubsection{Conclusion}

In conclusion, the fingerprint SERS peak at $374 \mathrm{~cm}^{-1}$ obtained from $\mathrm{Au} @ \mathrm{MBA}-\mathrm{Hg}^{2+}$ assay can be used to sensitively quantify the $\mathrm{Hg}^{2+}$ concentration with a LOD of 5 ppt. Good selectivity for $\mathrm{Hg}^{2+}$ was achieved using a masking agent of PDCA. The detection scheme based on the MBAfunctionalized $\mathrm{Au}$ NPs provided a new simple and effective approach for development of SERS sensors without Raman labels. 


\section{Reference}

1. Aragay, G.; Pons, J.; Merkoçi, A., Recent Trends in Macro-, Micro-, and NanomaterialBased Tools and Strategies for Heavy-Metal Detection. Chem Rev 2011, 111 (5), 3433-3458.

2. Li, M.; Gou, H. L.; Al-Ogaidi, I.; Wu, N. Q., Nanostructured Sensors for Detection of Heavy Metals: A Review. Acs Sustain Chem Eng 2013, 1 (7), 713-723.

3. Grandjean, P.; Landrigan, P. J., Developmental neurotoxicity of industrial chemicals. Lancet 2006, 368 (9553), 2167-2178.

4. $\quad$ Chen, K.; She, S.; Zhang, J. W.; Bayaguud, A.; Wei, Y. G., Label-free colorimetric detection of mercury via $\mathrm{Hg} 2+$ ions-accelerated structural transformation of nanoscale metal-oxo clusters. Sci Rep-Uk 2015, 5.

5. Xue, X. J.; Wang, F.; Liu, X. G., One-step, room temperature, colorimetric detection of mercury (Hg2+) using DNA/nanoparticle conjugates. J Am Chem Soc 2008, 130 (11), 3244-+.

6. Yu, C. J.; Tseng, W. L., Colorimetric Detection of Mercury(II) in a High-Salinity Solution Using Gold Nanoparticles Capped with 3-Mercaptopropionate Acid and Adenosine Monophosphate. Langmuir 2008, 24 (21), 12717-12722.

7. Chen, L.; Li, J. H.; Chen, L. X., Colorimetric Detection of Mercury Species Based on Functionalized Gold Nanoparticles. Acs Appl Mater Inter 2014, 6 (18), 15897-15904.

8. Chen, G. H.; Chen, W. Y.; Yen, Y. C.; Wang, C. W.; Chang, H. T.; Chen, C. F., Detection of Mercury(II) Ions Using Colorimetric Gold Nanoparticles on Paper-Based Analytical Devices. Anal Chem 2014, 86 (14), 6843-6849.

9. Du, Y. X.; Liu, R. Y.; Liu, B. H.; Wang, S. H.; Han, M. Y.; Zhang, Z. P., SurfaceEnhanced Raman Scattering Chip for Femtomolar Detection of Mercuric Ion (II) by Ligand Exchange. Anal Chem 2013, 85 (6), 3160-3165.

10. Ma, W.; Sun, M.; Xu, L.; Wang, L.; Kuang, H.; Xu, C., A SERS active gold nanostar dimer for mercury ion detection. Chem Commun 2013, 49 (44), 4989-4991.

11. Huang, C. C.; Chang, H. T., Parameters for selective colorimetric sensing of mercury(II) in aqueous solutions using mercaptopropionic acid-modified gold nanoparticles. Chem Commun 2007, (12), 1215-1217.

12. Zheng, P.; Li, M.; Jurevic, R.; Cushing, S. K.; Liu, Y. X.; Wu, N. Q., A gold nanohole array based surface-enhanced Raman scattering biosensor for detection of silver(I) and mercury(II) in human saliva. Nanoscale 2015, 7 (25), 11005-11012.

13. Yang, S. K.; Dai, X. M.; Stogin, B. B.; Wong, T. S., Ultrasensitive surface-enhanced Raman scattering detection in common fluids. P Natl Acad Sci USA 2016, 113 (2), 268-273.

14. Kneipp, K.; Kneipp, H.; Itzkan, I.; Dasari, R. R.; Feld, M. S., Ultrasensitive chemical analysis by Raman spectroscopy. Chem Rev 1999, 99 (10), 2957-2976.

15. Jackson, J. B.; Halas, N. J., Surface-enhanced Raman scattering on tunable plasmonic nanoparticle substrates. P Natl Acad Sci USA 2004, 101 (52), 17930-17935. 
16. Zheng, P.; Cushing, S. K.; Suri, S.; Wu, N. Q., Tailoring plasmonic properties of gold nanohole arrays for surface-enhanced Raman scattering. Phys Chem Chem Phys 2015, 17 (33), 21211-21219.

17. Liu, M.; Wang, Z. Y.; Zong, S. F.; Chen, H.; Zhu, D.; Wu, L.; Hu, G. H.; Cui, Y. P., SERS Detection and Removal of Mercury(II)/Silver(I) using Oligonucleotide-Functionalized Core/Shell Magnetic Silica Sphere@Au Nanoparticles. Acs Appl Mater Inter 2014, 6 (10), 73717379 .

18. Norkus, E.; Stalnioniene, I.; Crans, D. C., Interaction of pyridine- and 4hydroxypyridine-2,6-dicarboxylic acids with heavy metal ions in aqueous solutions. Heteroatom Chem 2003, 14 (7), 625-632.

19. Gilfrich, J. V.; Birks, L. S., Estimation of detection limits in x-ray fluorescence spectrometry. Anal Chem 1984, 56 (1), 77-79. 


\subsection{A SERS Sensor for Nitrite Detection}

\subsubsection{Introduction}

Nutrient pollution, which primarily originates from extensive usage of fertilizers and livestock waste, pose a threat on the ecological system. ${ }^{1-3}$ It was estimated that $1 \times 10^{11} \mathrm{~kg}$ of reactive nitrogen were released annually from nitrogen fertilizers around the world in the last decade, and ended up in aquatic systems in the form of nitrogen-containing compounds such as nitrite. $^{4-6}$ Nitrite is accountable for the rampant growth of eutrophication and algae in water bodies. $^{7-8}$ It can enter the food chain, and is taken by human. It is found to be correlated with the cause of methemoglobinemia in infants. Therefore, it is imperative to monitor nitrite in aquatic systems.

A common method for nitrite screening is Griess Test, which is based on the formation of a red pink color after the reaction between Griess reagents and the nitrite sample. ${ }^{9-11}$ However, the colorimetric nature makes Griess Test vulnerable to interferences from colored sample matrices, and suffer from poor sensitivity. Nitrite can also be measured quantitatively using chromatography methods, ${ }^{12-13}$ which act as the golden standard and is sensitive to part-per-billion level in drinking water. But considering the sophisticated operational procedure, high cost and immobility of ion chromatography, it is not suitable for routine monitoring of nitrite in field. Nowadays, efforts have been devoted to the development of inexpensive, rapid, portable and user-friendly sensors based on electrochemistry and fluorescence. ${ }^{14-21}$ However, the reported sensors show high noise levels and limited sensitivity.

It is well known that combination of the sensing signal labels/reporters with molecular recognition probes is required for most types of prevailing sensors in order to capture specific analytes and to transduce the sensing signal. For example, for detection of antigens or small molecule analytes, antibodies or aptamers are usually used as the molecular recognition probes to capture antigens or small molecule analytes; and fluorescent labels or redox probes are typically used to transduce the sensing signal. Compared to colorimetric, electrochemical and fluorescent sensors, the SERS devices can recognize the molecular spectral fingerprints of analytes and/or transduce the sensing signal by directly acquiring the SERS spectrum of analytes. ${ }^{22-26}$ This unique feature of SERS not only enables high selectivity and strong resistance to the interference in the complex sample matrix, but also may eliminate the use of the sensing signal labels/reporters or/and the molecular recognition probes in devices, which simplifies the design of sensors, and saves the cost. SERS sensors have been widely used for biomarker detection, environmental pollutant monitoring, drug and explosive measurement, and etc. ${ }^{27-29}$ Currently, SERS sensors build on the plasmonic nanostructures as the SERS substrates, such as Au and Ag in the form of colloidal nanoparticles or two-dimensional (2D) nanoarray pattern-based chips. ${ }^{30-31}$ The $2 \mathrm{D}$ plasmonic 
nanoarray pattern-based chips are of particular interest because they are able to generate "hot spots" in a large space, amplifying the SERS signal. ${ }^{32-35}$ However, it still remains a significant challenge in massive production of reproducible 2D plasmonic nanoarray pattern-based chips as the SERS substrate. ${ }^{36-37}$

In the present work, a facile SERS sensor has been developed for nitrite detection by coupling gold nanostars onto an ordered silver nanopyramid array pattern. The sensor transduces the SERS spectral fingerprints of an azo group, which is formed only in the presence of nitrite in the assay, allowing detection of nitrite without the aid of any antibody or aptamer. In the meanwhile, the plasmonic coupling between the Au nanostars and the Ag nanopyramid array pattern generates the intense "hot spots", enormously amplifying the SERS intensity of the newly formed azo group. Furthermore, the long-range ordered array of Ag nanopyramid makes it possible to amplify the SERS signals reproducibly. All these combined features of the SERS sensor not only improve the sensitivity toward nitrite detection, but also enable selectivity and reproducibility.

\subsubsection{Methods}

\section{Chemicals and Materials}

Polystyrene microspheres (diameters of 500, 600, and $1000 \mathrm{~nm}$ ) were purchased from Thermo Scientific. Sodium hydrobromide (99\%), polyvinylpyrrolidone (average molecular weight 10,000), N,N-dimethylformamide (ACS reagent, $\geq 99.8 \%$ ), 4-aminothiophenol (97\%), 1naphthylamine $(\geq 99.0 \%$ ), sodium nitrite (ACS reagent, $\geq 97.0 \%$ ), and hydrochloric acid (36.5$38.0 \%$ ) were purchased from Sigma-Aldrich. Chloroauric acid trihydrate (ACS, 99.99\%) and trisodium citrate dehydrate (ACS, 90.0+\%) were purchased from Alfa Aesar. Quartz slides were purchased from AdValue Technology. River water sample was collected from Monongahela River near Evansdale Campus of West Virginia University in Morgantown, West Virginia. DI water was produced by Milli-Q Millipore system (18.2 M $\Omega$ cm, Millipore Corp., Billerica, MA) and was used for washing and reactions. All chemicals were directly obtained from commercial vendors and used without further purification.

\section{Ag Nanopyramid Array Fabrication}

The Ag nanopyramid array patterns were fabricated on the quartz slides (roughly $1 \mathrm{~cm} \times 1$

$\mathrm{cm}$ ) in the cleanroom by nanosphere lithography, ${ }^{38-39}$ as shown in Figure 3.10. Specifically, the quartz slides were first cleaned by immersing into acid piranha (the ratio of sulfuric acid to hydrogen peroxide is $3: 1$ ) at $90{ }^{\circ} \mathrm{C}$ for 1 hour. Caution: acid piranha is very dangerous and needs to be handled with extreme care! The quartz slides were then rinsed thoroughly using deionized (D.I.) water, and further cleaned three times by consecutive sonication in ethanol and D.I. water, respectively. Afterwards, a monolayer of polystyrene microsphere (PS) was dip-coated on the quartz slides in a hexagonal pattern. The diameter of PS was varied from $500 \mathrm{~nm}$ and 600 to 1000 $\mathrm{nm}$ to tune the optical response of the resulting pyramid array. After that, a $5 \mathrm{~nm}$ thick titanium 
layer and a $200 \mathrm{~nm}$ thick silver film were deposited onto the quartz slides by e-beam evaporator. The PS beads were removed by sonication in methanol for five minutes and blown to dry using compressed air, resulting in the hexagonally ordered Ag nanopyramid array patterns.

(a) Quartz Cleaning

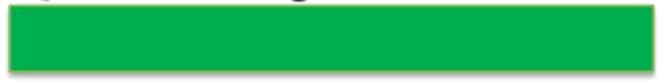

(b) Polystyrene Beads Patterning

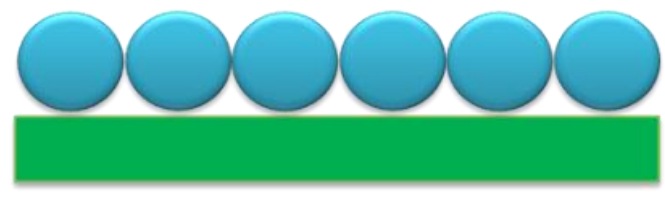

(c) $5 \mathrm{~nm}$ Titanium Layer Deposition

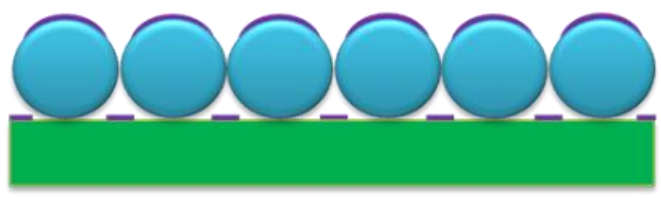

(d) $200 \mathrm{~nm}$ Silver Layer Deposition

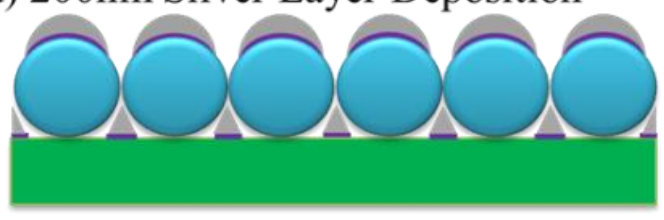

(f) Polystyrene Beads Removal

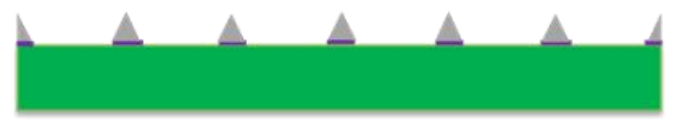

(h) Schematic Ag Nanopyramid Array

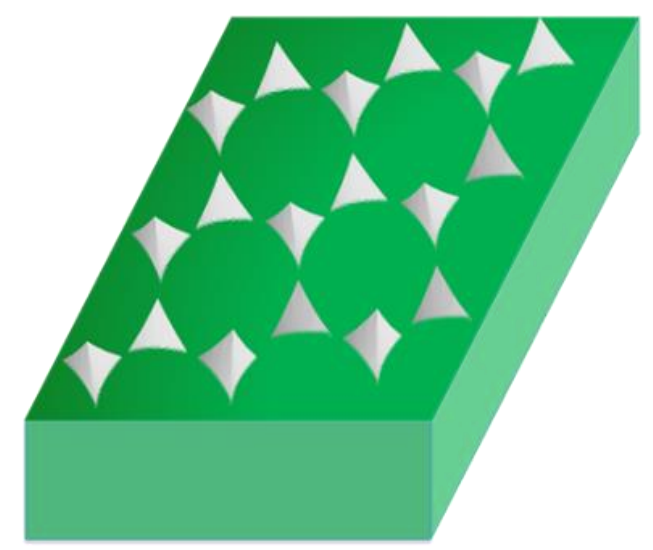

Figure 3.10 Protocol for Ag nanopyramid array fabrication.

\section{Au Nanostar Synthesis}

The Au nanostars were synthesized using a two-step approach reported previously. ${ }^{38}$ To begin with, the Au nanoseeds were first synthesized. $1 \mathrm{~mL}$ of $1 \mathrm{wt} \%$ chloroauric acid was first added to $90 \mathrm{~mL}$ of D.I. water, followed by $2 \mathrm{~mL}$ of $38.8 \mathrm{mM}$ trisodium citrate solution. Afterwards, $1 \mathrm{~mL}$ of $0.075 \mathrm{wt} \%$ sodium hydrobromide solution was added into the above solution and kept reacting overnight. Subsequently, polyvinylpyrrolidone was dissolved into a $50 \mathrm{~mL}$ of $\mathrm{Au}$ nanoseed solution; and the mixture was stirred for 24 hours. In order to synthesize the Au nanostars, $82 \mu \mathrm{L}$ of $50 \mathrm{mM}$ chloroauric acid was added into a $15 \mathrm{~mL}$ of $10 \mathrm{mM}$ polyvinylpyrrolidone solution, which was followed by the addition of $43 \mu \mathrm{L}$ of Au nanoseeds. The mixture was stirred overnight before being centrifuged and washed using ethanol and water. The washing and centrifugation steps were repeated three times before the obtained Au nanostars were finally dissolved into ethanol.

\section{Instruments and Characterization}

Titanium and silver were deposited using an e-beam evaporator (Kurt $\mathrm{J}$ Lesker, Model\#LAB18). Ag nanopyramid arrays were characterized under a JEOL JSM-7600F scanning electron microscope (SEM). The Au nanostars were characterized using a JEOL JEM-2100F transmission electron microscope (TEM). An Ocean Optics USB 4000 spectrometer was used to 
acquire the reflection spectra of the fabricated Ag nanopyramid arrays. Raman spectra were acquired using the iRaman plus (Model\# BWS465, B\&W Tek) with an excitation wavelength of $785 \mathrm{~nm}$.

\section{FDTD Simulation}

Optical properties of the Ag nanopyramid array patterns, the Au nanostars were studied using finite different time domain (FDTD) simulation. FDTD software is commercially available from Optiwave Systems Inc. A grid size of $1 \mathrm{~nm}$ was used to construct the simulation cell. A plane wave with a center wavelength of $600 \mathrm{~nm}$ was used as the input light source. The wavelength-dependent refractive index of silver was taken from Palik. ${ }^{40}$ The refractive index of quartz slides was modeled as a constant of 1.53. Periodic boundary conditions were applied for all simulations.

\section{SERS Measurement}

The Au nanostars were first functionalized with 4-aminothiophenol (4-ATP). Specifically, 10 $\mathrm{uL}$ of $10 \mathrm{mM}$ 4-ATP in ethanol was added into $10 \mathrm{~mL}$ of Au nanostars. The mixture was stirred overnight before being centrifuged and washed three times to remove excessive 4-ATP molecules using D.I. water. It was then dissolved in D.I. water, resulting in the Au star@ATP nanoparticles. The pH of Au star@ATP nanoparticle solution was adjusted to 3.5 using 2 M hydrochloric acid.

The Ag nanopyramid array patterns were functionalized with 1-naphthylamine (1-NA). Specifically, an $\mathrm{Ag}$ nanopyramid array was incubated into $10 \mathrm{~mL}$ of $10 \mathrm{mM}$ 1-NA ethanolic solution overnight, forming Ag nanopyramid@NA. It was then rinsed using ethanol to remove excessive 1-NA molecules and dried using compressed air.

Standard nitrite solutions with concentrations of $1 \mathrm{pg} / \mathrm{mL}, 10 \mathrm{pg} / \mathrm{mL}, 100 \mathrm{pg} / \mathrm{mL}, 1 \mathrm{ng} / \mathrm{mL}$, $10 \mathrm{ng} / \mathrm{mL}, 100 \mathrm{ng} / \mathrm{mL}, 1 \mu \mathrm{g} / \mathrm{mL}, 10 \mu \mathrm{g} / \mathrm{mL}, 100 \mu \mathrm{g} / \mathrm{mL}$, and $1 \mathrm{mg} / \mathrm{mL}$ were prepared by dissolving sodium nitrite into D.I. water, respectively.

In order to calibrate the sensor for nitrite detection, $5 \mu \mathrm{L}$ of standard nitrite solutions was first mixed with $50 \mu \mathrm{L}$ of Au nanostar@ATP solution. The mixed solution was immediately dispensed onto the surface of a Ag nanopyramid@NA substrate. After 10 minutes of incubation, the Ag nanopyramid@NA was washed using D.I. water and dried using compressed air. SERS spectra were then acquired from the surface of the Ag nanopyramid array substrate. In this process, the laser power was set at $10 \%$ of the full power $(>320 \mathrm{~mW}$ at laser port, $420 \mathrm{~mW}$ Max from the vendor) with an integration exposure time of $10 \mathrm{~s}$ for each measurement.

To detect nitrite in river water, the water sample was first treated by natural sedimentation for a week, and then further purified by filtration using a filter paper prior to measurement. The procedure for SERS measurement was the same as above. 


\subsubsection{Results and Discussions}

\section{Optical Properties of Ag Nanopyramid Array and Au Nanostars}

Fabricated by nanosphere lithography ${ }^{38-39}$ with the procedure shown in Figure 3.10, the Ag nanopyramid arrays exhibited a tetrahedron shape with three sharp edges and a needle-like tip at the apex, as shown in Figure 3.11(a) and (b). 67 objectives were measured for the Ag nano pyramids based on the SEM images. The average size of Ag nano pyramids was $\sim 207 \mathrm{~nm}$ in height and $\sim 120 \mathrm{~nm}$ in base length with the standard deviations of $17 \mathrm{~nm}$ and $18 \mathrm{~nm}$. Based on Mie theory, the overall extinction of a metal particle includes the contributions of both light absorption and scattering. A plasmonic particle can absorb or scatter light when localized surface plasmon resonance (LSPR) is excited by incident light, which is dependent on the particle size. When the particle is small (typically less than $50 \mathrm{~nm}$ ), light absorption is dominant while light scattering is negligible. When the particle is large (for example, $100 \mathrm{~nm}$ in a diameter), light scattering is strong while absorption is weak. Given that the Ag nanopyramid was quite large ( $200 \mathrm{~nm}$ in height and $\sim 120 \mathrm{~nm}$ in base length), light scattering was dominant in the present work.

(a)

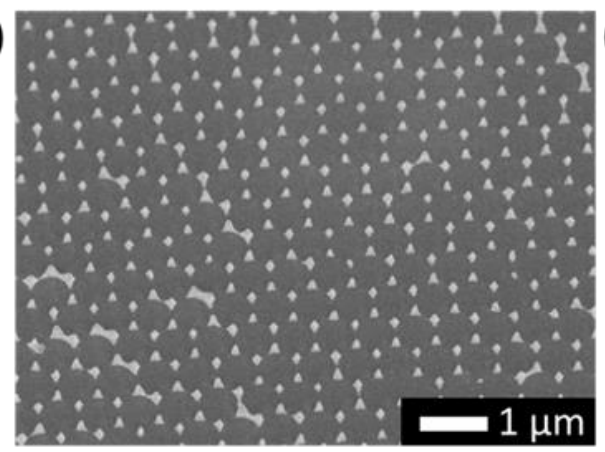

(c)

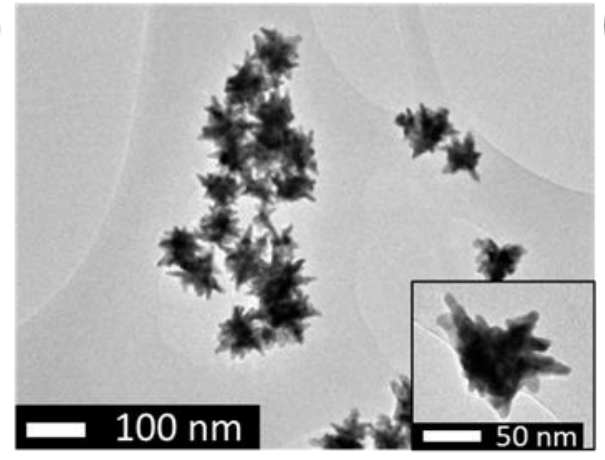

(b)

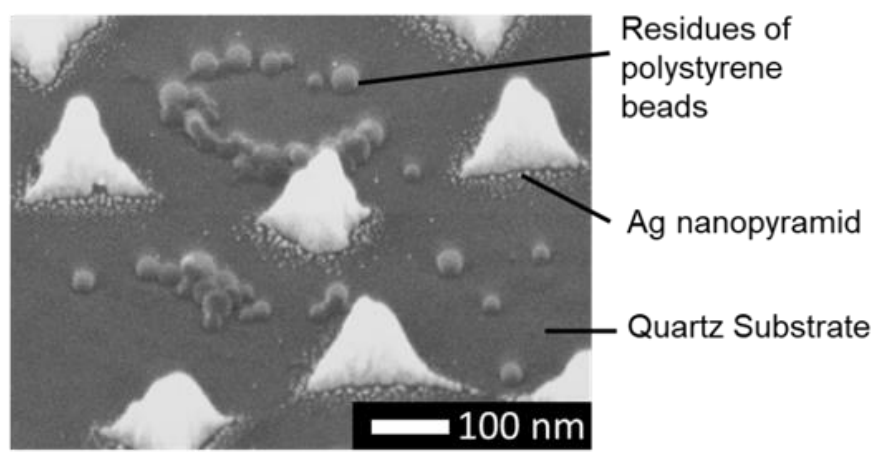

(d)

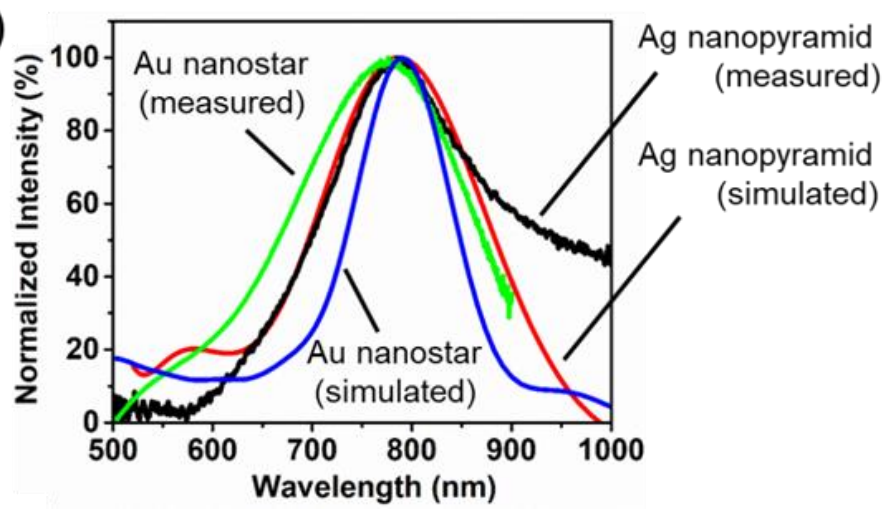

Figure 3.11 (a) and (b) SEM images of the Ag nanopyramid array pattern; (c) TEM image of the Au nanostars (inset shows a single Au nanostar); (d) Measured and simulated back reflection spectra for the Ag nanopyramid array pattern and absorption spectra for the Au nanostars. The back reflection and absorption intensities in (d) have been normalized for comparision. 
25 objectives were measured for the Au nanostars based on the SEM and TEM images. The average size of $\mathrm{Au}$ nanostars was $80 \mathrm{~nm}$ with a standard deviation of $6 \mathrm{~nm}$. The Au nanostars showed an average size of $80 \mathrm{~nm}$, as shown in Figure 3.11(c). Despite the difference in shape, the Au nanostars and the Ag nanopyramid array pattern exhibited similar surface plasmon resonance spectral features with both the peaks centered at around $785 \mathrm{~nm}$, which was also confirmed by the calculated UV-Visible spectra, as shown in Figure 3.11(d). The consistency between the simulated and the measured spectra implied predictability of optical properties as well as controllability of the obtained geometries, allowing design of the optimal SERS substrates with aid of finitedifference time-domain (FDTD) simulation. Furthermore, the spectral overlap suggested an intense electron wave function interaction, leading to plasmonic coupling between the Ag nanopyramid array and the Au nanostar under a $785 \mathrm{~nm}$ laser excitation.

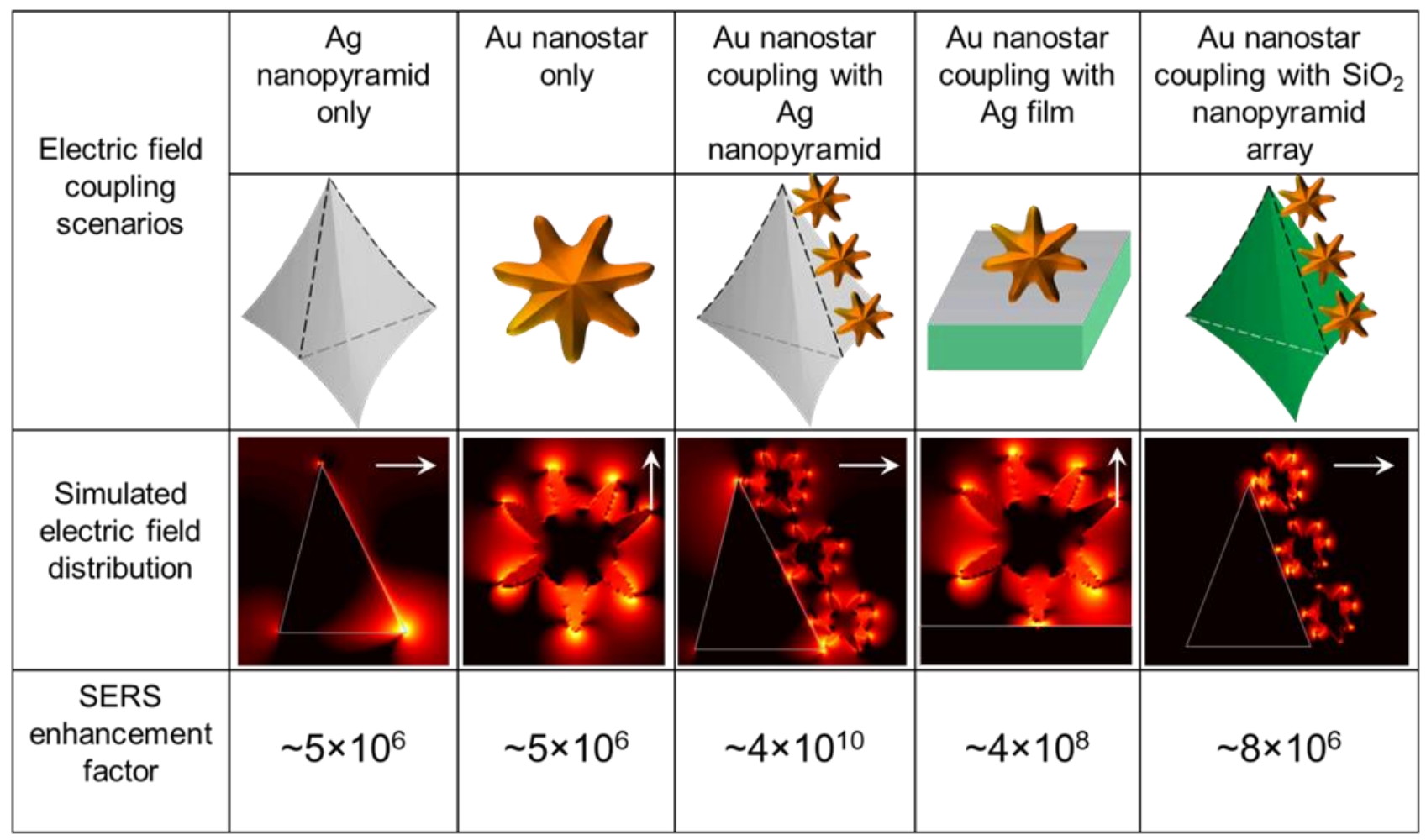

Figure 3.12 (a) Simulated electromagnetic field distribution and SERS enhancement factor for various coupling scenarios between a silver nanopyramid and a gold nanostar with an excitation wavelength of $785 \mathrm{~nm}$. The gap distance was set as $1 \mathrm{~nm}$ for the Au nanostar coupled with the silver nanopyramid, or with a silver film, with a $\mathrm{SiO}_{2}$ nanopyramid array. The arrows in the simulated electric field distribution indicate the polarization directions

Prior to sensor construction, FDTD simulation was implemented to study plasmon modes as well as plasmonic coupling, which could give an insight of the origin of the electromagnetic enhancement. We modelled the scenario of an $\mathrm{Au}$ nanostar sitting on one face of an $\mathrm{Ag}$ 
nanopyramid (Figure 3.12), which is also the most probable coupling case. The separation distance between the Au nanostar and the Ag nanopyramid was set as $1 \mathrm{~nm}$, which is the approximate length of the newly formed azo compound bridging the Ag nanopyramid and the Au nanostar, as shown in Figure 3.13. The input plane wave was polarized in a way to enable the strongest plasmonic coupling. The simulated electromagnetic field distribution suggested that the Ag nanopyramid array predominately displayed vertex and edge plasmon modes, whereas the Au nanostar exhibited a tip plasmon mode. Upon plasmonic coupling, intense gap plasmon modes were generated at the $1 \mathrm{~nm}$ gap between an Au nanostar and an $\mathrm{Ag}$ nanopyramid, which was exactly the origin of the electromagnetic field enhancement. The SERS enhancement factor consisting of an excitation enhancement $\left(\left|\mathbf{E}_{\mathbf{7 8 5}}\right| / \mathbf{E}_{\mathbf{7 8 5}} \mathbf{0}^{0}\right)^{2}$ and the emission enhancement $\left(\left|\mathbf{E}_{\mathbf{8 6 2}}\right| / \mathbf{E}_{\mathbf{8 6 2}} \mathbf{0}^{0} \mid\right)^{2}$ was also calculated, where $\mathbf{E}_{785}$ and $\mathbf{E}_{\mathbf{8 6 2}}$ are the EM field at the excitation wavelength $(785 \mathrm{~nm})$ and the Stokes-shifted wavelength $(862 \mathrm{~nm})$ for the coupling normalized by the EM field at incident wavelengths $\mathbf{E}_{\mathbf{7 8 5}}{ }^{\mathbf{0}}$ and $\mathbf{E s 6 2}^{\mathbf{}}$, respectively. It was found that an SERS enhancement factor $\left(\left|\mathbf{E}_{\mathbf{7 8 5}}\right| / \mathbf{E}_{\mathbf{7 8 5}} \mathbf{0}^{0}\right)^{2} \cdot\left(\left|\mathbf{E}_{\mathbf{8 6 2}}\right| / \mathbf{E}_{\mathbf{8 6 2}}{ }^{0} \mid\right)^{2}$ of $\sim 4 \times 10^{10}$ was achieved when an Au nanostar sitting on the face of an Ag nanopyramid. That is, the Raman intensity of the molecules at the gap would be amplified $\sim 4 \times 10^{10}$ times. As a comparison, a factor of $\sim 5 \times 10^{6}$ was achieved for either an individual $\mathrm{Au}$ nanostar or an individual Ag nanopyramid.

\section{Operating Mechanism of Sensor}

As shown in Figure 3.13(a), when the nitrite ions are present in the assay, the diazotization reaction between 1-naphthylamine (1-NA) and 4-aminothiophenol (4-ATP) happens, ${ }^{9-10}$ producing an azo compound which exhibited the SERS fingerprints. This eliminates the use of any Raman reporter in the SERS sensor design for detection of nitrite. The SERS sensor consisted of the Ag nanopyramid array pattern and the Au nanostars, which are initially functionalized with 1-NA and 4-ATP, respectively, as shown in Figure 3.13(b). The nitrite ions first react with the amine group of 4-ATP under an acidic condition, leading to the formation of diazonium compounds. The diazonium compounds further react with 1-NA, producing the azo compounds. As a result, the $\mathrm{Au}$ nanostars are covalently connected to the Ag nanopyramid array via the formation of the azo group. These newly formed azo group not only displays a SERS spectral fingerprint, but also benefits from the intense EM field enhancement thanks to the "hot spots" between the Ag nanopyramid and the Au nanostars. The mechanism was verified by the SERS spectra in Figure 3.14(a). The newly formed azo groups exhibited at least three new SERS peaks at $1140 \mathrm{~cm}^{-1}, 1389 \mathrm{~cm}^{-1}$, and $1434 \mathrm{~cm}^{-1}$ as compared to the 4-ATP functionalized Au nanostars and the 1-NA functionalized Ag nanopyramid array. The uniformity of the substrate was also studied by detecting the SERS signal at 15 random locations across the 1-NA functionalized Ag nanopyramid array. The relative standard deviation (RSD) was found to be $6.6 \%$. Furthermore, the SERS sensor was tested separately against the possibly interfering inorganic compounds such as $\mathrm{NaNO}_{3}, \mathrm{NaCl}, \mathrm{Na}_{2} \mathrm{SO}_{4}$, $\mathrm{KCl}$, and $\mathrm{K}_{3} \mathrm{PO}_{4}$. As shown in Figure 3.14(b), the SERS sensor barely responded to any of the above inorganic compounds. Since the new SERS peaks were solely related to the azo group formed by the diazotization reaction in the presence of nitrite. This unique feature can significantly improve the signal-to-noise ratio, allowing the SERS sensor to specifically detect nitrite with 
minimal interference. In addition, unlike other SERS-based immunoassays or DNA-based SERS biosensors which heavily rely on expensive biological reagents such as antibodies and DNA, this SERS sensor employed only two small molecules (1-NA and 4-ATP). This makes the sensor costeffective without compromising the performance.

(a) Reaction

$$
\hbar v
$$$$
\hbar v^{\prime}
$$<smiles>Nc1cccc2ccccc12</smiles><smiles>Nc1ccc(S)cc1</smiles><smiles>Nc1ccc(N=Nc2ccc(S)cc2)c2ccccc12</smiles>

\section{Raman scattering}

(b) Detection scheme
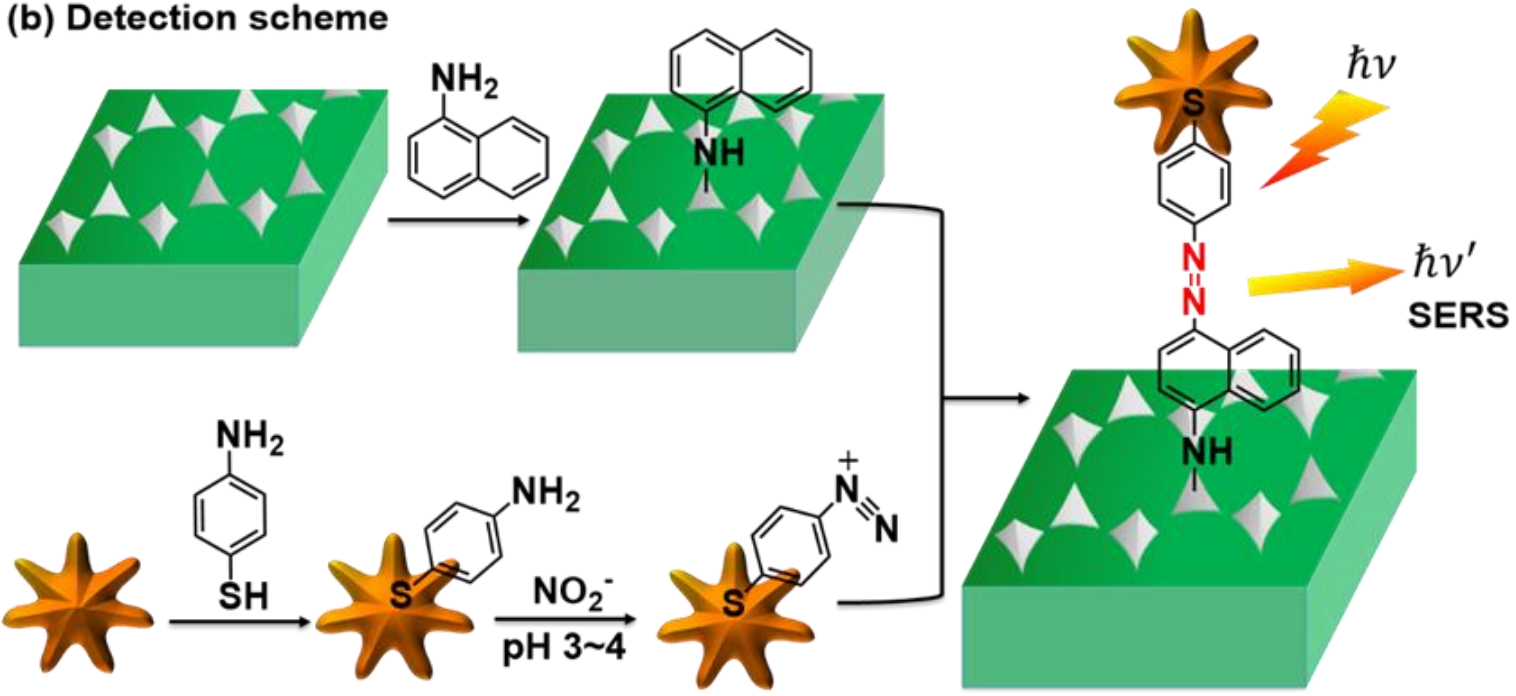

Figure 3.13 Mechanism of nitrite detection with the SERS sensor. (a) Reaction: 1-naphthylamine (1-NA) and 4-aminothiophenol (4-ATP) reacts in the presence of nitrite ions under acidic condition, generating an azo compound with a SERS fingerprint (marked using a red color); (b) Detection scheme: the 1-NA functionalized Ag nanopyramid array captures the 4-ATP functionalized Au nanostar via formation of the azo compound. 


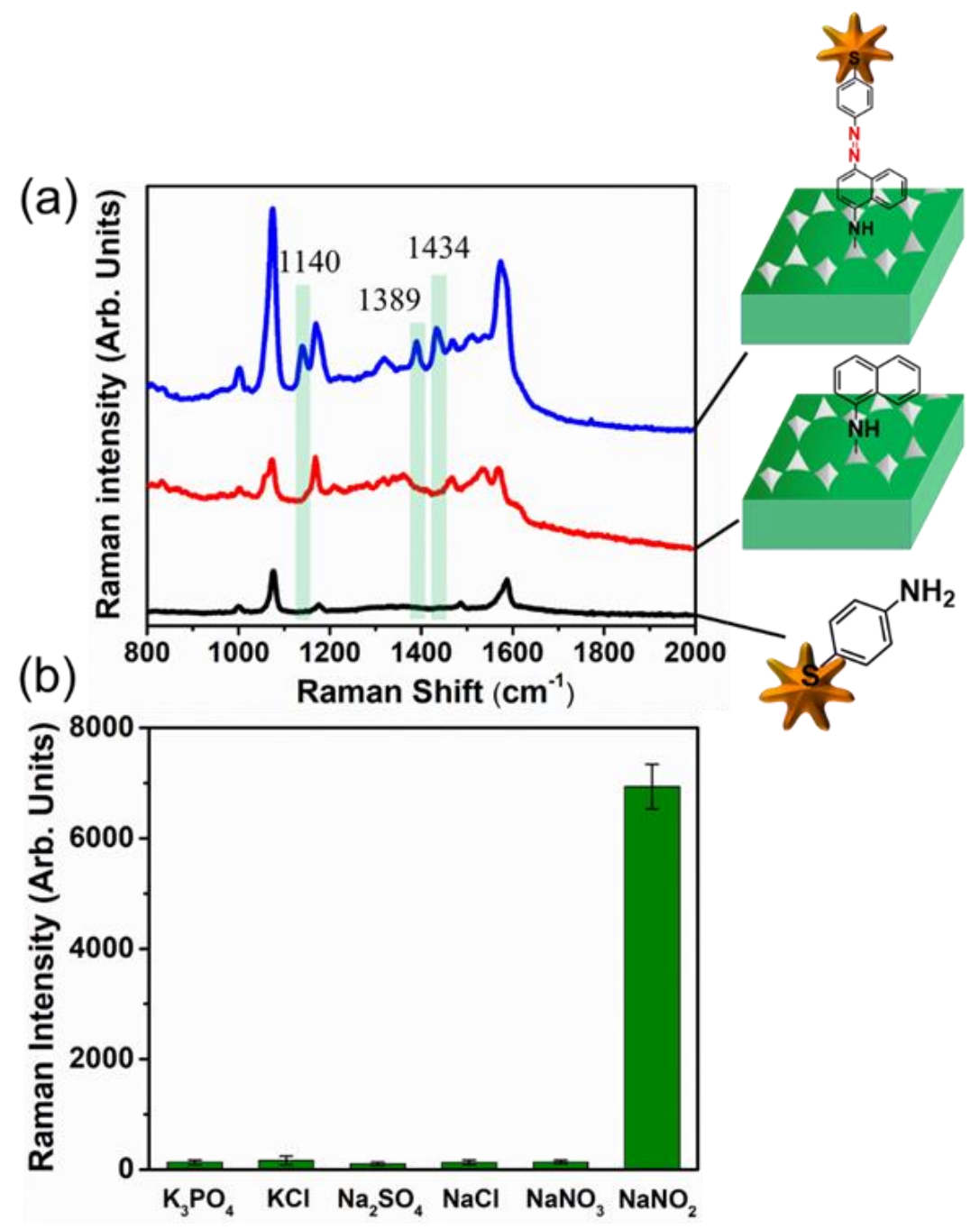

Figure 3.14 (a) SERS spectra comparison: the new azo compound produces three characteristic SERS peaks from the azo group at $1140 \mathrm{~cm}^{-1}, 1389 \mathrm{~cm}^{-1}$, and $1434 \mathrm{~cm}^{-1}$ as compared to the 1-NA functionalized Ag nanopyramid array and the 4-ATP functionalized Au nanostar; (b) Selectivity test: possibly interfering compounds $\mathrm{K}_{3} \mathrm{PO}_{4}, \mathrm{KCl}, \mathrm{Na}_{2} \mathrm{SO}_{4}, \mathrm{NaCl}$, and $\mathrm{NaNO}_{3}$ were tested separately but resulted in barely detectable SERS peak at $1140 \mathrm{~cm}^{-1}$.

\section{Calibration of SERS Sensor}

The SERS sensor was calibrated using the standard nitrite solutions. The concentration ranged from $1 \mathrm{pg} / \mathrm{mL}$ to $1 \mathrm{mg} / \mathrm{mL}$ with an interval of 1 order of magnitude. It is noted that the SERS spectra in Figure 3.15(a) were intentionally offset for a better visualization of the spectra evolution. After the addition of a nitrite solution, three new SERS peaks at $1140 \mathrm{~cm}^{-1}, 1389 \mathrm{~cm}^{-1}$, and 1434 $\mathrm{cm}^{-1}$ showed up as expected (Figure 3.15(a)). The SERS intensity became intensified with an increase in the concentration of nitrite. All these three peaks can be used for quantification of the nitrite concentration based on the statistical analysis. Because the $1140 \mathrm{~cm}^{-1}$ peak responded to nitrite most sensitively although the difference among the three characteristic SERS peaks is 
marginal as shown in Figure 3.16, it was selected to build the calibration curve for nitrite detection. The SERS intensity of the $1140 \mathrm{~cm}^{-1}$ peak was plotted as a function of the logarithmic concentration of nitrite (Figure 3.15(b)), which showed a continuous SERS intensity increase till saturation with increasing the nitrite concentration. In the linear response region, the calibration curve was fitted with an equation of $y=703 x+868, R^{2}=99 \%$, where $y$ is the measured intensity of the SERS peak at $1140 \mathrm{~cm}^{-1}, x$ is the logarithmic concentration of nitrite, as shown in Figure 3.15(c). The limit of detection (LOD) was calculated to be $0.6 \mathrm{pg} / \mathrm{mL}$ based on three times signal-to-noise ratio. The SERS sensor showed lower LOD than the common techniques used for nitrite detection, such as chromatography and Griess test. The LODs are typically 1.6 75 ppb and 23 115 ppb for chromatography $y^{41-42}$ and Griess test ${ }^{43}$, respectively. Such a low LOD for the SERS sensor resulted from the SERS enhancement factor of $\sim 10^{10}$, demonstrating high sensitivity of the SERS sensor. The SERS sensor also displayed an extended linear detection range spanning from $1 \mathrm{pg} / \mathrm{mL}$ to 10 $\mathrm{ug} / \mathrm{ml}$. This will endow the sensor with a wide range of applications for detection of either trace amount of nitrite or heavily accumulated nitrite pollutant in river, lake, pond, and etc. It is noteworthy that U.S. Environmental Protection Agency (EPA) regulates that the maximum contaminant level (MCL) for nitrite in drinking water is $1.0 \mu \mathrm{g} / \mathrm{mL}$, which falls into the linear detection range of the SERS sensor. In addition, the selection of a single characteristic SERS peak at $1140 \mathrm{~cm}^{-1}$ to build the calibration curve was further justified by comparing with the full spectrum analysis using principle component analysis (PCA), as shown in Figure 3.17, where the response of the SERS sensor to nitrite concentration variation is very similar. Comparison of the response of the SERS sensor to varying nitrite concentrations between using the single SERS peak at 1140 $\mathrm{cm}^{-1}$ and using the full spectrum analysis by PCA was also shown in Figure 3.18, where the difference is marginal. 

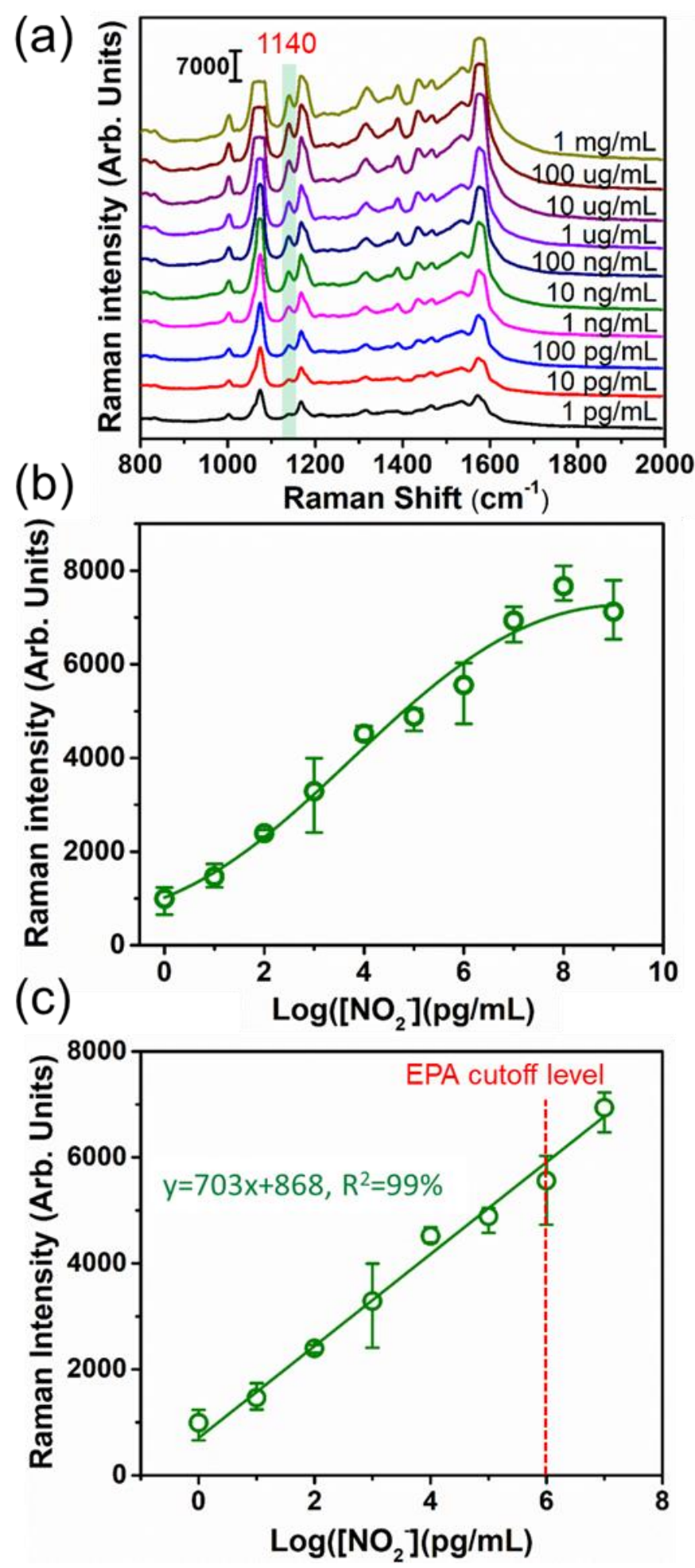

Figure 3.15 SERS sensor calibration. (a) SERS spectra obtained at various concentrations of nitrite; (b) Plot of the intensity at $1140 \mathrm{~cm}^{-1}$ versus the logarithmic concentration of nitrite; (c) Fitting of the linear region in (b); The red dashed line shows the Maximum Contaminant Level of nitrite in drinking water regulated by EPA. 


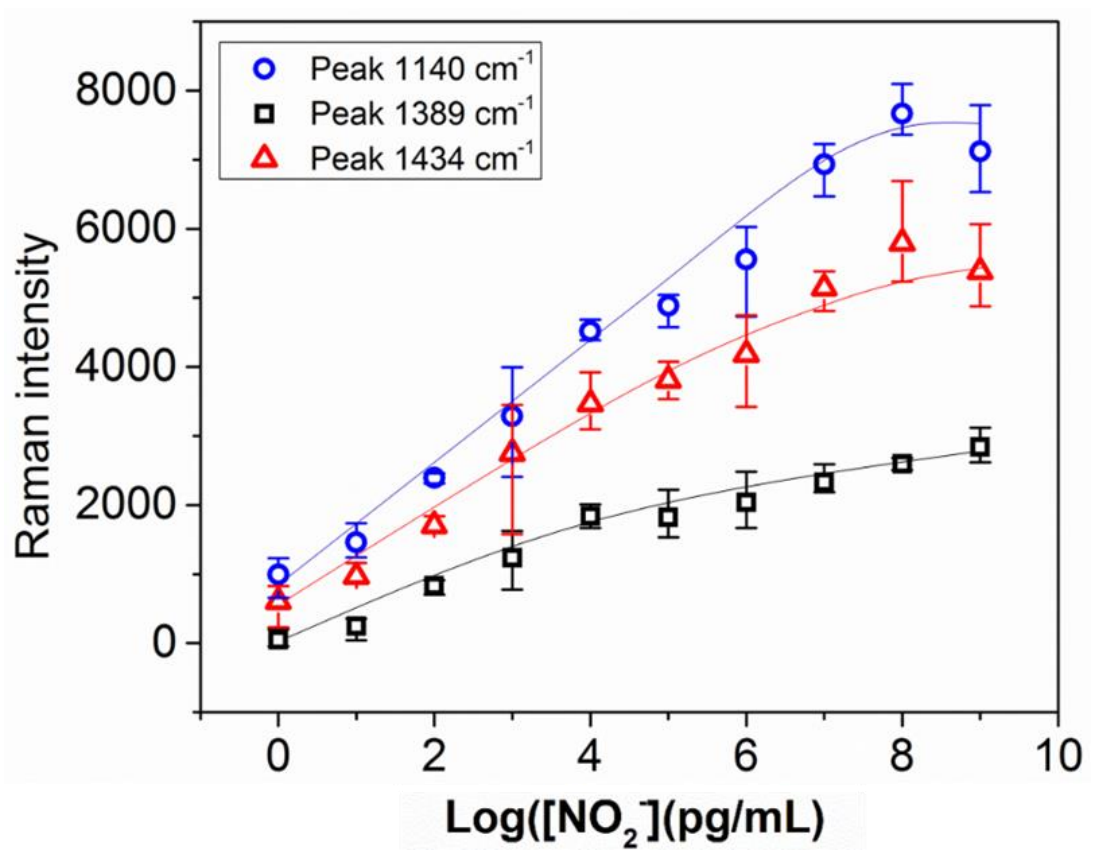

Figure 3.16 Fitting curves for different SERS peaks at $1140 \mathrm{~cm}^{-1}, 1389 \mathrm{~cm}^{-1}$, and $1434 \mathrm{~cm}^{-1}$. The SERS peak at $1140 \mathrm{~cm}^{-1}$ gives the most sensitive response for nitrite detection. 


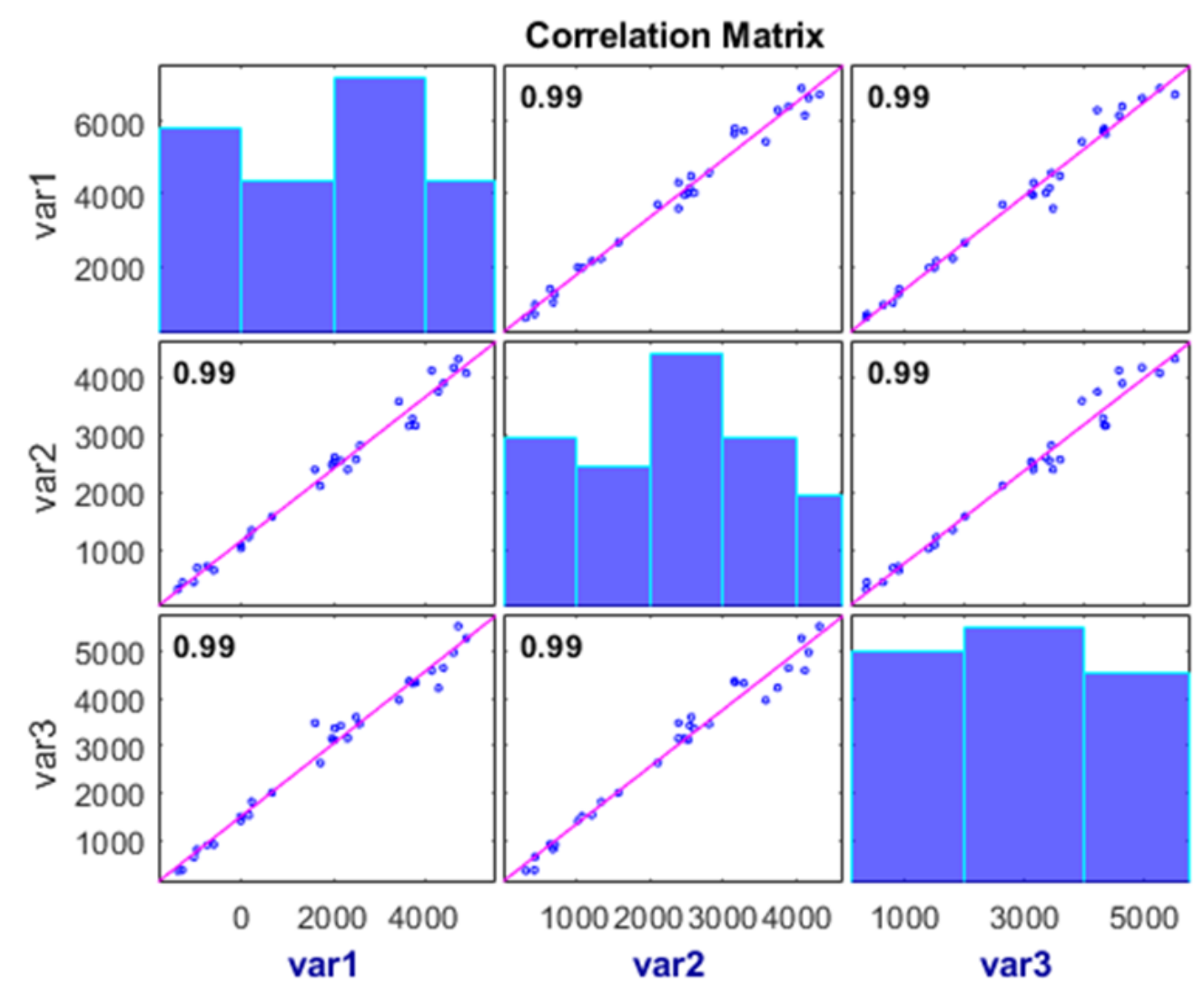

Figure 3.17 Correlation matrix for the three characteristic SERS peaks at $1140 \mathrm{~cm}^{-1}(\operatorname{var} 1), 1389 \mathrm{~cm}^{-1}(\operatorname{var} 2)$, and $1434 \mathrm{~cm}^{-1}(\operatorname{var} 3)$. The intensities of the three Raman shifts are identified as the potential predictors for the concentration of the target analyte. The pairwise correlations between the intensities at any two of the three Raman shifts have indicated that each intensity pair is nearly perfectly positively correlated with a correlation estimate of 0.99 , which is graphically illustrated by the linear plots. The nearly perfect correlations suggest that the intensities at the three Raman shifts play nearly equivalent roles in the prediction of analyte concentrations. 

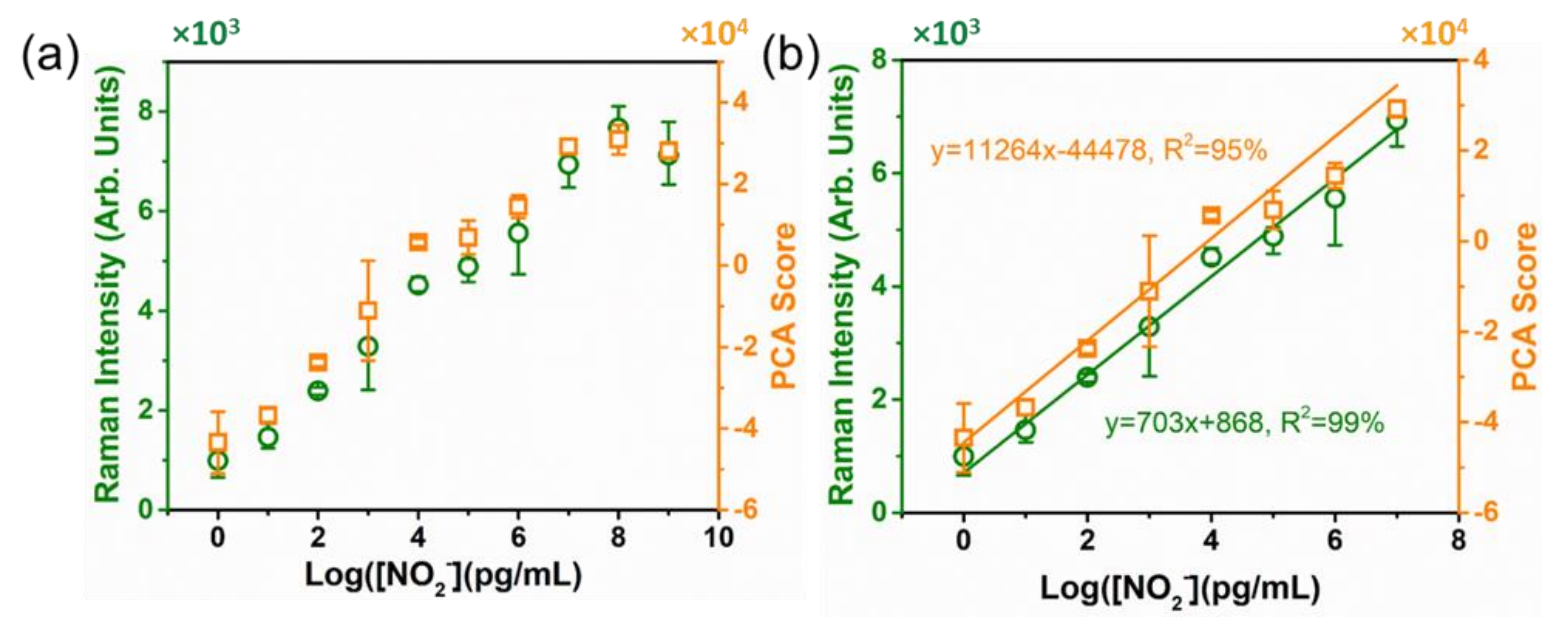

Figure 3.18 Comparison of the response of the SERS sensor to varying nitrite concentrations between using the single SERS peak at $1140 \mathrm{~cm}^{-1}$ (olive color) and using the full spectrum analysis by principal component analysis (PCA, orange color). (a) includes all the studied nitrite concentrations; (b) includes the nitrite concentrations in the linear response region. The difference between using a single SERS peak at $1140 \mathrm{~cm}^{-1}$ and using full spectrum analysis by PCA is marginal.

\section{Detection of Nitrite in River Water}

The SERS sensor was used to test the water samples taken from Monongahela River near the Evansdale campus of West Virginia University in Morgantown, West Virginia. It was treated by natural sedimentation and filtration prior to testing by the SERS sensor. After acquisition of 19 SERS spectra from the sample, the nitrite level in river water sample was calculated according to the equation of the calibration curve: $y=703 x+868$. It was found to be $10^{5.94 \pm 0.58} \mathrm{pg} / \mathrm{mL}$ (a mean value of $0.87 \mu \mathrm{g} / \mathrm{mL}$ with an upper limit of $3.3 \mu \mathrm{g} / \mathrm{mL}$ and a lower limit of $0.23 \mu \mathrm{g} / \mathrm{mL}$ ) with a relative standard deviation (RSD) of $8.4 \%$. In comparison, the sample was also tested by EPA 300.0 Ion Chromatography (Exova Inc), where the nitrite concentration was found to be 1.00 $\mu \mathrm{g} / \mathrm{mL}$, which was consistent with the result obtained by the SERS sensor.

In short, a SERS sensor developed in the present work is highly sensitive and selective. The detection scheme developed in this work can be adapted for measuring other ions and small molecules. For rapid and convenient measurement of nitrite and small molecules, it is desirable to incorporate a sensor into a microfluidic chip to achieve automation, sample handling and friendly user interface. For promoting the SERS sensor development, optical readers of SERS sensor need to be further improved in terms of size and cost.

\subsubsection{Conclusion}

In summary, an ordered silver nanopyramid array pattern was successfully fabricated with nanosphere lithography. By coupling the gold nanostars to the silver nanopyramid array pattern, a 
SERS sensor was built to detection nitrite. The presence of nitrite not only created three unique SERS peaks at $1140 \mathrm{~cm}^{-1}, 1389 \mathrm{~cm}^{-1}$, and $1434 \mathrm{~cm}^{-1}$ from the azo group, but also resulted in the formation of "hot spots" where the azo group were located at the gap between the gold nanostar and the silver nanopyramid. This unique sensing mechanism eliminated the use of any aptamer or antibody and enabled high sensitivity of the SERS sensor. As a result, the SERS sensor exhibited a LOD of $0.6 \mathrm{pg} / \mathrm{mL}$ toward nitrite detection in deionized water. The capability of the SERS sensor was demonstrated for nitrite detection in river water. The result showed that the SERS sensor was highly sensitive, selective and inexpensive; and it possessed unique advantages over the colorimetric, electrochemical and fluorescent devices for small molecule detection. 


\section{Reference}

1. Socolow, R. H., Nitrogen management and the future of food: Lessons from the management of energy and carbon. Proceedings of the National Academy of Sciences 1999, 96 (11), 6001.

2. Woodward, G.; Gessner, M. O.; Giller, P. S.; Gulis, V.; Hladyz, S.; Lecerf, A.; Malmqvist, B.; McKie, B. G.; Tiegs, S. D.; Cariss, H.; Dobson, M.; Elosegi, A.; Ferreira, V.; Graça, M. A. S.; Fleituch, T.; Lacoursière, J. O.; Nistorescu, M.; Pozo, J.; Risnoveanu, G.; Schindler, M.; Vadineanu, A.; Vought, L. B. M.; Chauvet, E., Continental-Scale Effects of Nutrient Pollution on Stream Ecosystem Functioning. Science 2012, 336 (6087), 1438.

3. Shibata, H.; Branquinho, C.; McDowell, W. H.; Mitchell, M. J.; Monteith, D. T.; Tang, J.; Arvola, L.; Cruz, C.; Cusack, D. F.; Halada, L.; Kopáček, J.; Máguas, C.; Sajidu, S.; Schubert, H.; Tokuchi, N.; Záhora, J., Consequence of altered nitrogen cycles in the coupled human and ecological system under changing climate: The need for long-term and site-based research. Ambio 2015, 44 (3), 178-193.

4. Chen, J.; Pang, S.; He, L.; Nugen, S. R., Highly sensitive and selective detection of nitrite ions using Fe3O4@SiO2/Au magnetic nanoparticles by surface-enhanced Raman spectroscopy. Biosensors and Bioelectronics 2016, 85, 726-733.

5. Maryna, S.; Lin, M.; Zhaohai, B.; Shengji, L.; Carolien, K.; Oene, O.; Gerard, V.; Fusuo, Z., Alarming nutrient pollution of Chinese rivers as a result of agricultural transitions.

Environmental Research Letters 2016, 11 (2), 024014.

6. Fields, S., Global Nitrogen: Cycling out of Control. Environmental Health Perspectives 2004, 112 (10), A556-A563.

7. Luo, Y.; Wen, G.; Dong, J.; Liu, Q.; Liang, A.; Jiang, Z., SERS detection of trace nitrite ion in aqueous solution based on the nitrosation reaction of rhodamine $6 \mathrm{G}$ molecular probe. Sensors and Actuators B: Chemical 2014, 201, 336-342.

8. Ing Hua, T.; Rita, S.; Hendrik, O. L.; Leny, Y., Detection of nitrite and nitrate ions in water by graphene oxide as a potential fluorescence sensor. IOP Conference Series: Materials Science and Engineering 2016, 107 (1), 012027.

9. Daniel, W. L.; Han, M. S.; Lee, J.-S.; Mirkin, C. A., Colorimetric Nitrite and Nitrate Detection with Gold Nanoparticle Probes and Kinetic End Points. J Am Chem Soc 2009, 131 (18), 6362-6363.

10. Correa-Duarte, M. A.; Pazos Perez, N.; Guerrini, L.; Giannini, V.; Alvarez-Puebla, R. A., Boosting the Quantitative Inorganic Surface-Enhanced Raman Scattering Sensing to the Limit: The Case of Nitrite/Nitrate Detection. The Journal of Physical Chemistry Letters 2015, 6 (5), 868-874.

11. Giustarini, D.; Rossi, R.; Milzani, A.; Dalle-Donne, I., Nitrite and Nitrate Measurement by Griess Reagent in Human Plasma: Evaluation of Interferences and Standardization. In Methods in Enzymology, Academic Press: 2008; Vol. 440, pp 361-380. 
12. Kissner, R.; Koppenol, W. H., Qualitative and Quantitative Determination of Nitrite and Nitrate with Ion Chromatography. In Methods in Enzymology, Academic Press: 2005; Vol. 396, pp 61-68.

13. Di Matteo, V.; Esposito, E., Methods for the determination of nitrite by high-performance liquid chromatography with electrochemical detection. Journal of Chromatography A 1997, 789 (1), 213-219.

14. Zou, C. e.; Yang, B.; Bin, D.; Wang, J.; Li, S.; Yang, P.; Wang, C.; Shiraishi, Y.; Du, Y., Electrochemical synthesis of gold nanoparticles decorated flower-like graphene for high sensitivity detection of nitrite. Journal of Colloid and Interface Science 2017, 488, 135-141.

15. Cai, M.; Chai, X.; Wang, X.; Wang, T., An Acid-Inert Fluorescent Probe for the Detection of Nitrite. Journal of Fluorescence 2017, 27 (4), 1365-1371.

16. Chen, J.; Ma, Q.; Wang, C.; Hu, X.; Gao, Y.; Wang, H.; Qin, D.; Lu, X., A simple fluorescence sensor for the detection of nitrite (NO2-) in real samples using water-dispersible graphite-like carbon nitride (w-g-C3N4) nanomaterials. New Journal of Chemistry 2017, 41 (15), 7171-7176.

17. Li, L.; Liu, D.; Wang, K.; Mao, H.; You, T., Quantitative detection of nitrite with N-doped graphene quantum dots decorated N-doped carbon nanofibers composite-based electrochemical sensor. Sensors and Actuators B: Chemical 2017, 252, 17-23.

18. Wang, P.; Wang, M.; Zhou, F.; Yang, G.; Qu, L.; Miao, X., Development of a paperbased, inexpensive, and disposable electrochemical sensing platform for nitrite detection. Electrochemistry Communications 2017, 81, 74-78.

19. Su, C.-H.; Sun, C.-L.; Liao, Y.-C., Printed Combinatorial Sensors for Simultaneous Detection of Ascorbic Acid, Uric Acid, Dopamine, and Nitrite. Acs Omega 2017, 2 (8), 42454252.

20. Davis, J.; Moorcroft, M. J.; Wilkins, S. J.; Compton, R. G.; Cardosi, M. F., Electrochemical detection of nitrate and nitrite at a copper modified electrode. Analyst 2000, 125 (4), 737-742.

21. Wang, P.; Mai, Z.; Dai, Z.; Li, Y.; Zou, X., Construction of Au nanoparticles on choline chloride modified glassy carbon electrode for sensitive detection of nitrite. Biosensors and Bioelectronics 2009, 24 (11), 3242-3247.

22. Sharma, B.; Frontiera, R. R.; Henry, A.-I.; Ringe, E.; Van Duyne, R. P., SERS: Materials, applications, and the future. Materials Today 2012, 15 (1), 16-25.

23. Sigle, D. O.; Kasera, S.; Herrmann, L. O.; Palma, A.; de Nijs, B.; Benz, F.; Mahajan, S.; Baumberg, J. J.; Scherman, O. A., Observing Single Molecules Complexing with Cucurbituril through Nanogap Surface-Enhanced Raman Spectroscopy. The Journal of Physical Chemistry Letters 2016, 7 (4), 704-710.

24. Zrimsek, A. B.; Wong, N. L.; Van Duyne, R. P., Single Molecule Surface-Enhanced Raman Spectroscopy: A Critical Analysis of the Bianalyte versus Isotopologue Proof. The Journal of Physical Chemistry C 2016, 120 (9), 5133-5142. 
25. Wang, F.; Cao, S.; Yan, R.; Wang, Z.; Wang, D.; Yang, H., Selectivity/Specificity Improvement Strategies in Surface-Enhanced Raman Spectroscopy Analysis. Sensors 2017, 17 (1424-8220 (Electronic)), 2689.

26. Petti, L.; Rippa, M.; Capasso, R.; Zhou, J.; Maglione, M. G.; Pannico, M.; La Manna, P.; Musto, P., Plasmonic octagonal quasicrystals for surface enhanced Raman sensing. Advanced Device Materials 2015, 1 (2), 47-51.

27. Schlücker, S., Surface-Enhanced Raman Spectroscopy: Concepts and Chemical Applications. Angewandte Chemie International Edition 2014, 53 (19), 4756-4795.

28. Muehlethaler, C.; Leona, M.; Lombardi, J. R., Review of Surface Enhanced Raman Scattering Applications in Forensic Science. Anal Chem 2016, 88 (1), 152-169.

29. Park, S.-G.; Ahn, M.-S.; Oh, Y.-J.; Kang, M.; Jeong, Y.; Jeong, K.-H., Nanoplasmonic biopatch for in vivo surface enhanced raman spectroscopy. BioChip Journal 2014, 8 (4), 289294.

30. Li, J. F.; Huang, Y. F.; Ding, Y.; Yang, Z. L.; Li, S. B.; Zhou, X. S.; Fan, F. R.; Zhang, W.; Zhou, Z. Y.; Wu, D. Y.; Ren, B.; Wang, Z. L.; Tian, Z. Q., Shell-isolated nanoparticle-enhanced Raman spectroscopy. Nature 2010, 464, 392.

31. Wei, X.; Sebastian, S., Rationally designed multifunctional plasmonic nanostructures for surface-enhanced Raman spectroscopy: a review. Reports on Progress in Physics 2014, 77 (11), 116502.

32. Lin, T.-H.; Linn, N. C.; Tarajano, L.; Jiang, B.; Jiang, P., Electrochemical SERS at Periodic Metallic Nanopyramid Arrays. The Journal of Physical Chemistry C 2009, 113 (4), 1367-1372.

33. Jin, M.; Pully, V.; Otto, C.; van den Berg, A.; Carlen, E. T., High-Density Periodic Arrays of Self-Aligned Subwavelength Nanopyramids for Surface-Enhanced Raman Spectroscopy. The Journal of Physical Chemistry C 2010, 114 (50), 21953-21959.

34. Tabatabaei, M.; Sangar, A.; Kazemi-Zanjani, N.; Torchio, P.; Merlen, A.; LagugnéLabarthet, F., Optical Properties of Silver and Gold Tetrahedral Nanopyramid Arrays Prepared by Nanosphere Lithography. The Journal of Physical Chemistry C 2013, 117 (28), 14778-14786.

35. Nicholas, C. L.; Chih-Hung, S.; Ajay, A.; Peng, J.; Bin, J., Surface-enhanced Raman scattering on periodic metal nanotips with tunable sharpness. Nanotechnology 2009, 20 (22), 225303.

36. Alonso-González, P.; Albella, P.; Schnell, M.; Chen, J.; Huth, F.; García-Etxarri, A.; Casanova, F.; Golmar, F.; Arzubiaga, L.; Hueso, L. E.; Aizpurua, J.; Hillenbrand, R., Resolving the electromagnetic mechanism of surface-enhanced light scattering at single hot spots. Nat Commun 2012, 3, 684.

37. Ding, S.-Y.; You, E.-M.; Tian, Z.-Q.; Moskovits, M., Electromagnetic theories of surfaceenhanced Raman spectroscopy. Chem Soc Rev 2017, 46 (13), 4042-4076.

38. Zheng, P.; Li, M.; Jurevic, R.; Cushing, S. K.; Liu, Y. X.; Wu, N. Q., A gold nanohole array based surface-enhanced Raman scattering biosensor for detection of silver(I) and mercury(II) in human saliva. Nanoscale 2015, 7 (25), 11005-11012. 
39. Haynes, C. L.; Van Duyne, R. P., Nanosphere Lithography: A Versatile Nanofabrication Tool for Studies of Size-Dependent Nanoparticle Optics. The Journal of Physical Chemistry B 2001, 105 (24), 5599-5611.

40. Palik, E. D., Handbook of Optical Constants of Solids. Elsevier Science: 1991.

41. Miura, Y.; Hamada, H., Ion chromatography of nitrite at the ppb level with photometric measurement of iodine formed by post-column reaction of nitrite with iodide. Journal of Chromatography A 1999, 850 (1), 153-160.

42. Ito, K.; Takayama, Y.; Makabe, N.; Mitsui, R.; Hirokawa, T., Ion chromatography for determination of nitrite and nitrate in seawater using monolithic ODS columns. Journal of Chromatography A 2005, 1083 (1), 63-67.

43. Shen, Y.; Zhang, Q.; Qian, X.; Yang, Y., Practical Assay for Nitrite and Nitrosothiol as an Alternative to the Griess Assay or the 2,3-Diaminonaphthalene Assay. Anal Chem 2015, 87 (2), 1274-1280. 


\section{Chapter 4: Plasmon-Enhanced Fluorescence for Sensing}

\subsection{Elucidating the Role of Scattering in Plasmon-Enhanced Fluorescence}

\subsubsection{Introduction}

Optimizing plasmon-enhanced fluorescence (PEF) requires a delicate balance between the excitation rate enhancement and the quantum efficiency enhancement. ${ }^{1-7}$ The excitation rate enhancement originates from the distance-dependent local electric field enhancement and drops exponentially when the quantum emitter moves away from the plasmonic nanostrucure. The quantum efficiency enhancement is decided by the extent to which the radiative and non-radiative decay rate are respectively modified under the influence of the environment. In other words, when the modification of the radiative decay rate outcompetes that of the non-radiative decay rate, the quantum efficiency is enhanced. At a very short separation distance, the quantum emitter primarily couples through non-radiative resonant energy transfer to non-radiating higher-order plasmon modes and thus ends up experiencing reduced quantum efficiency. ${ }^{8}$ As the separation distance increases, the quantum emitter becomes more likely to be coupled with the dipole mode which dominates the plasmon field. The radiating nature of the dipole resonance enables the quantum emitter to experience a less reduction in quantum efficiency when the intrinsic quantum efficiency is high and an enhancement of quantum efficiency when the intrinsic quantum efficiency is low. ${ }^{9-}$ ${ }^{10}$ Given the large variation of quantum efficiency even at a subtle change of the separation between the quantum emitter and the nanostructure, the separation distance is deemed as a critical parameter in optimizing PEF. ${ }^{4}$, 11-13 By continuously varying the separation distance, the observed fluorescence can be tuned to transit from quenching to enhancement.

An important parameter to consider in PEF is the Purcell effect. ${ }^{14}$ For an excited atomic state, the rate at which the atom spontaneously decays to the ground sate can be modified by tuning the local density of optical states. In other words, the spontaneous decay rate could be enhanced in the presence of additional optical modes, i.e. local density of optical states. This is made possible by spectrally and spatially coupling the quantum emitter to a cavity mode with a high Q-factor and a small modal volume. The increase of spontaneous decay rate is described by the Purcell factor and could lead to fluorescence enhancement provided that the modification of radiative component in 
the spontaneous decay rate outcompetes the non-radiative component. ${ }^{15-16}$

In addition to the separation distance between the quantum emitter and the nanostructure and the Purcell factor, scattering has recently been phenomenally demonstrated to contribute to or correlate with the fluorescence enhancement. ${ }^{17-25}$ Although the role of scattering on PEF has not yet been systematically elucidated, the consensus is that scattering could make the quantum emitter more likely to radiate to the far field by coupling to the nanostructure. For instance, gold nanoshells were found to lead to large quantum efficiency improvement than gold nanorods due to a larger scattering cross section at the emission wavelength of the quantum emitter. ${ }^{17-18}$

In this study, the role of scattering on PEF was systematically studied by means of finitedifference time-domain (FDTD) based numeric simulation. For simplicity, the quantum emitter was modelled as an electric point dipole and represented by a two-level system. An Ag nanosphere with varying diameter was used as the plasmonic nanostructure with tunable scattering coefficients. The transition frequency of the quantum emitter was fixed at the localized surface plasmon resonance (LSPR) peak wavelength of the Ag nanosphere. The local electromagnetic (EM) field enhancement $|\mathbf{E}|^{2} /\left|\mathbf{E}_{\mathbf{0}}\right|^{2}$, Purcell factor, radiative decay rate enhancement, quantum efficiency enhancement, and fluorescence enhancement were calculated with respect to the varying separation distance between the quantum emitter and the Ag nanosphere as well as the diameter of the Ag nanosphere. It was found that scattering play dual roles in modulating both the local EM field and enhancing the quantum efficiency. Further calculation of the correlation coefficients among these parameters established a clear correlation relation between scattering and fluorescence enhancement.

\subsubsection{Results and Discussions}

\section{Silver Nanosphere for Fluorescence Enhancement}

Since the contribution of scattering to extinction can be easily tuned by varying the diameter of an Ag nanosphere, the effect of scattering on PEF was studied by coupling a quantum emitter to an Ag nanosphere, as schematically shown in Figure 4.1(a). The quantum emitter was modelled as an electric point dipole and represented by a two-level system with a transition at the LSPR peak wavelength of the Ag nanosphere. As the Ag nanosphere diameter increased, the extinction transited from being dominated by absorption to scattering. At a diameter of approximately $55 \mathrm{~nm}$, the extinction was equally split by absorption and scattering. 
(a)

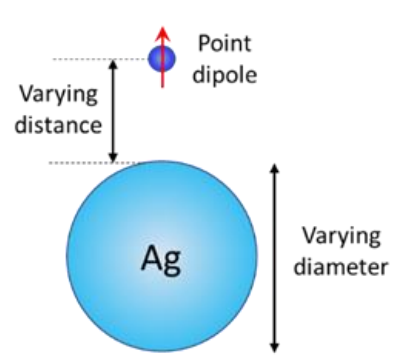

(d)

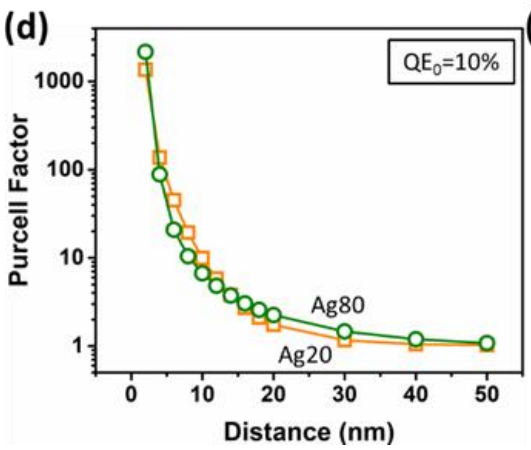

(b)
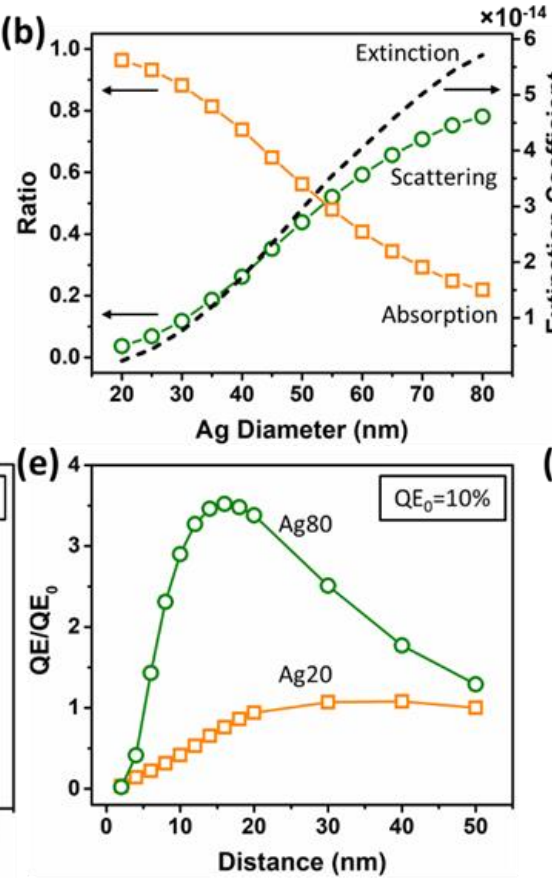

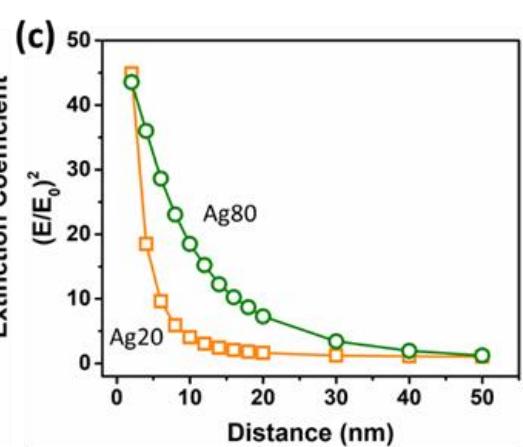

(f)

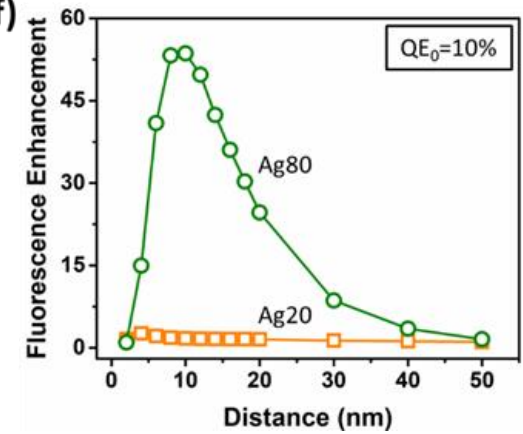

Figure 4.1 (a) Scheme showing a quantum emitter modelled as an electric point dipole was placed a varying distance away from the Ag nanosphere with a varying diameter; (b) The dependence of extinction, scattering, and absorption on the Ag nanosphere diameter; Comparison of the distancedependent (c) local EM field decay profile (d) Purcell factor (e) quantum efficiency enhancement and (f) fluorescence enhancement for $\mathrm{Ag}$ nanospheres with a diameter of $20 \mathrm{~nm}$ and $80 \mathrm{~nm}$. The intrinsic quantum efficiency was set as $10 \%$.

To clearly show the effect of scattering, Ag nanospheres with a diameter of $20 \mathrm{~nm}$ and $80 \mathrm{~nm}$ were compared and they were abbreviated as Ag20 and Ag80 for simplicity. While the diameter of the Ag nanosphere affected the local EM field to some degree, it barely made a difference to the Purcell factor, as shown in Figure 4.1(c) and (d). Although Ag80 had a larger contribution to the excitation rate enhancement than Ag20 in Figure 1(c), the correlation between scattering and fluorescence enhancement was not entirely caused by the effect of scattering on the excitation rate enhancement, but also by the effect of scattering on the quantum efficiency enhancement. As shown in Figure 4.1(e), Ag80 showed a larger quantum efficiency enhancement than Ag20 owing to the scattering effect, which was further manifested by the fluorescence enhancement in Figure 4.1(f). 

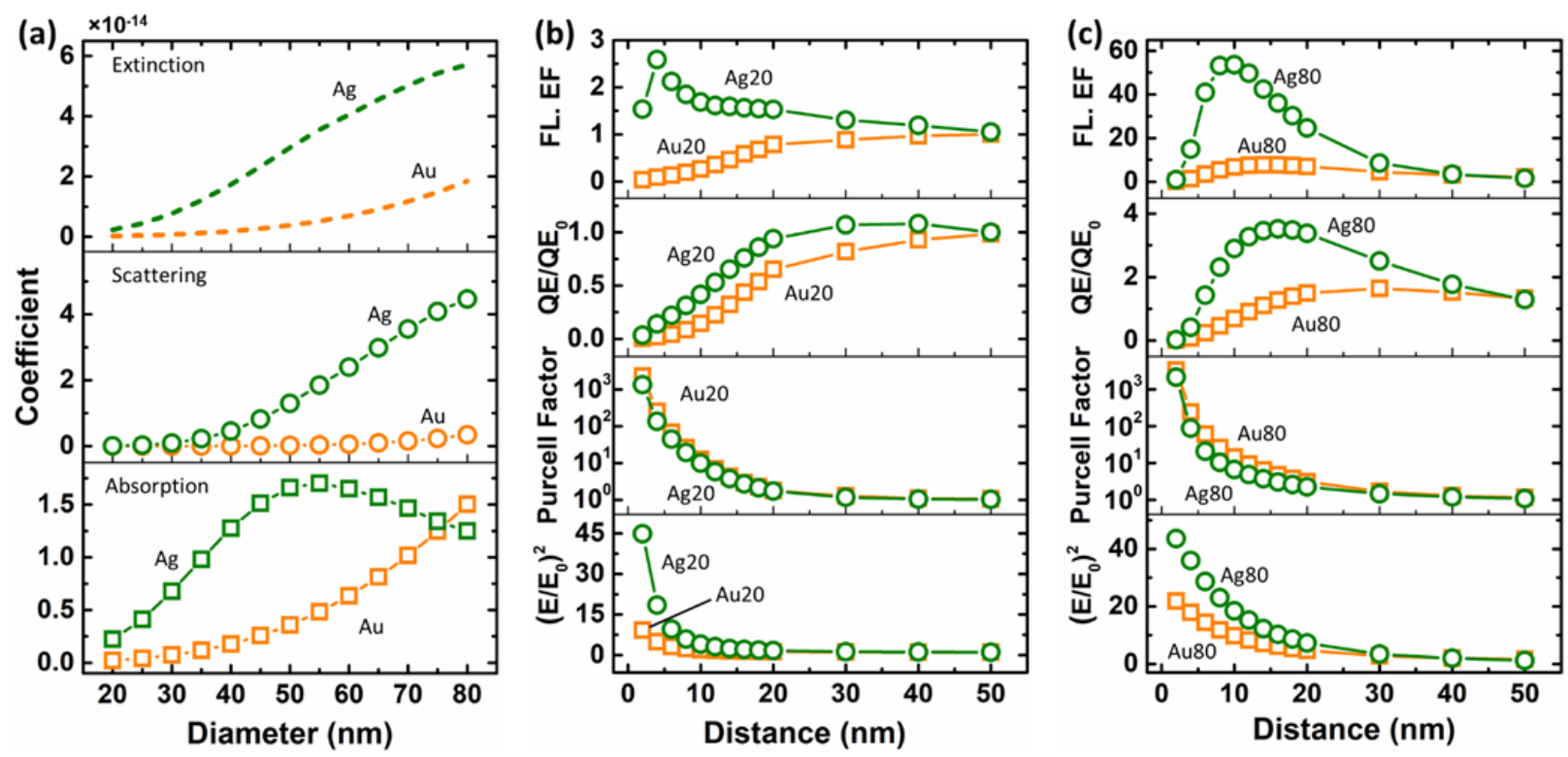

Figure 4.2 (a) Comparison of the extinction, scattering, and absorption coefficients between $\mathrm{Ag}$ and $\mathrm{Au}$ nanospheres; Comparison of fluorescence enhancement factor, quantum efficiency enhancement, Purcell factor, and local EM field enhancement between $\mathrm{Ag}$ and $\mathrm{Au}$ nanospheres with a diameter of (b) $20 \mathrm{~nm}$ and (c) $80 \mathrm{~nm}$.

\section{Comparison between Silver and Gold Nanospheres}

To understand whether the effect of scattering on PEF is a universal effect or just limited to the same type of material, Au nanospheres were studied under the same condition and compared with Ag nanospheres. At the smallest diameter considered, i.e. $20 \mathrm{~nm}$, the Ag nanosphere exhibited larger extinction, scattering, and absorption coefficients than the Au nanosphere although the difference was marginal. When the diameter increased, the Ag nanosphere displayed increasingly larger extinction and scattering coefficients than the Au nanosphere, as shown in Figure 4.2(a). It was observed that the Ag nanosphere always exhibited larger fluorescence enhancement and quantum efficiency enhancement than the Au nanosphere regardless of the size and distance, although the difference of Purcell factor and local EM field enhancement between them remained marginal, as shown in Figure 4.2(b) and (c). The results indicated that the effect of scattering on PEF was universal regardless of the materials. 

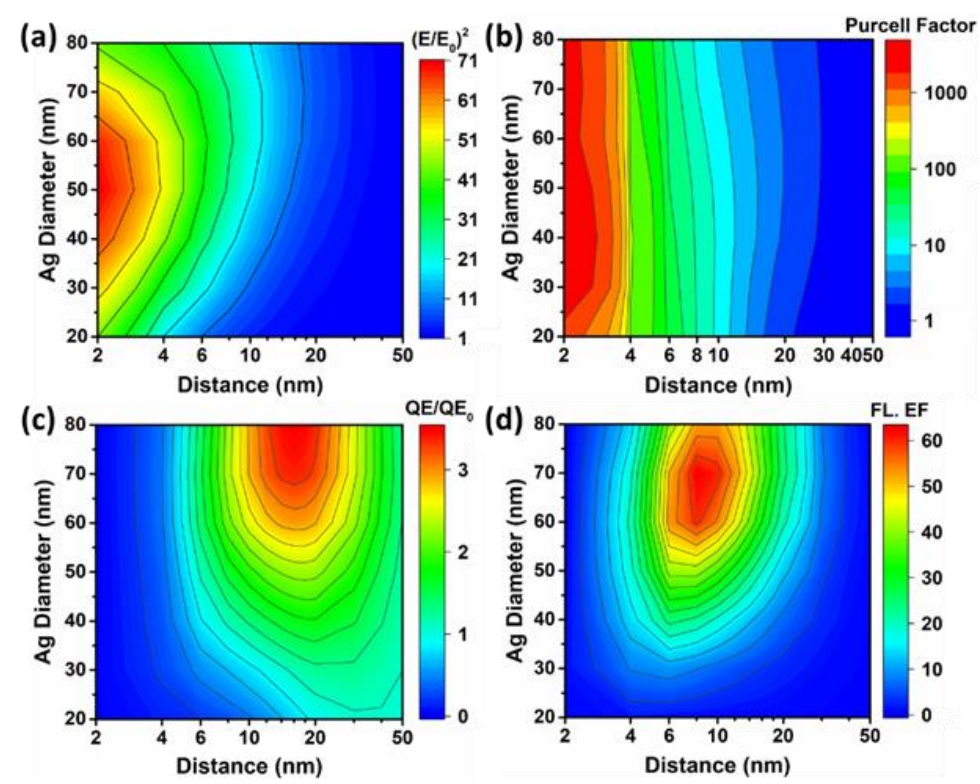

Figure 4.3 Systematic study of the effect of Ag nanosphere diameter and separation distance between the quantum emitter and the Ag nanosphere on (a) the local EM field enhancement (b) Purcell factor (c) quantum efficiency enhancement and (d) the fluorescence enhancement. The intrinsic quantum efficiency was set as $10 \%$. Log scale was applied to the distance for better visualization.

\section{Tuning Ag Nanosphere}

Having established an intuitive understanding that scattering plays an important role in PEF through modifying the local EM field and enhancing the quantum efficiency, we proceed to systematically tune the Ag nanosphere diameter and separation distance between the quantum emitter and the Ag nanosphere in order to reveal the correlation relations among different parameters. Although Ag80 showed a larger local EM field than Ag20 in Figure 4.1(c), the local EM field was found not to increase monotonically with respect to the Ag nanosphere diameter. Interestingly, a maximum local EM field was reached at a diameter of around $55 \mathrm{~nm}$, as shown in Figure 4.3(a). It is exactly the size at which absorption and scattering evenly split the extinction, indicating an equilibrium between the energy buildup and dissipation on the Ag nanosphere owing to the incoming excitation and radiative scattering. Different from the local EM field, the Purcell factor was barely size-dependent but decayed exponentially when the quantum emitter was moved away from the Ag nanosphere, as shown in Figure 4.3(b). Notably, the quantum efficiency enhancement exhibited a clear monotonic dependence on the Ag nanosphere diameter, or equivalently the scattering, as well as a separation distance dependence, as shown in Figure 4.3(c). The largest quantum efficiency enhancement could be found at a separation distance between 10 to $25 \mathrm{~nm}$. The fluorescence enhancement was also presented in Figure 4.3(d) by multiplying Figure 4.3(a) and (c), accounting for both the excitation rate enhancement and the quantum efficiency enhancement. The largest fluorescence enhancement could be found at a separation distance between 5 and $15 \mathrm{~nm}$. The slight decrease of the fluorescence enhancement when the Ag 
nanosphere diameter exceeded $70 \mathrm{~nm}$ coincided with the decrease of the local EM field owing to a large energy dissipation rate caused by the increased radiative scattering. Therefore, as a matter of fact, scattering played dual roles in PEF. On one hand, it contributed to the quantum efficiency by allowing the quantum emitter to radiate more efficiently to the far field. On the other hand, the competition between scattering and absorption set an upper limit to the achievable local EM field enhancement. The resulting fluorescence was thus decided by the modulation of scattering on the quantum efficiency as well as the local EM field enhancement.

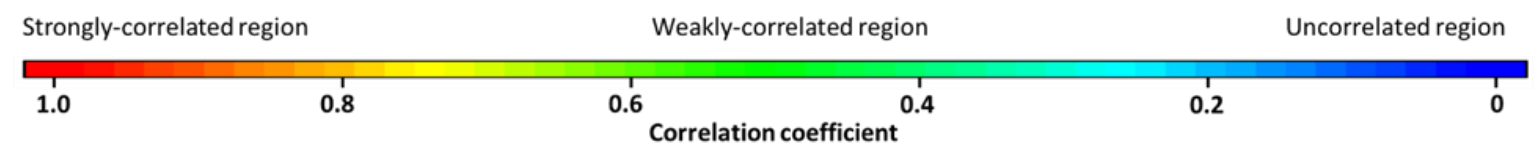
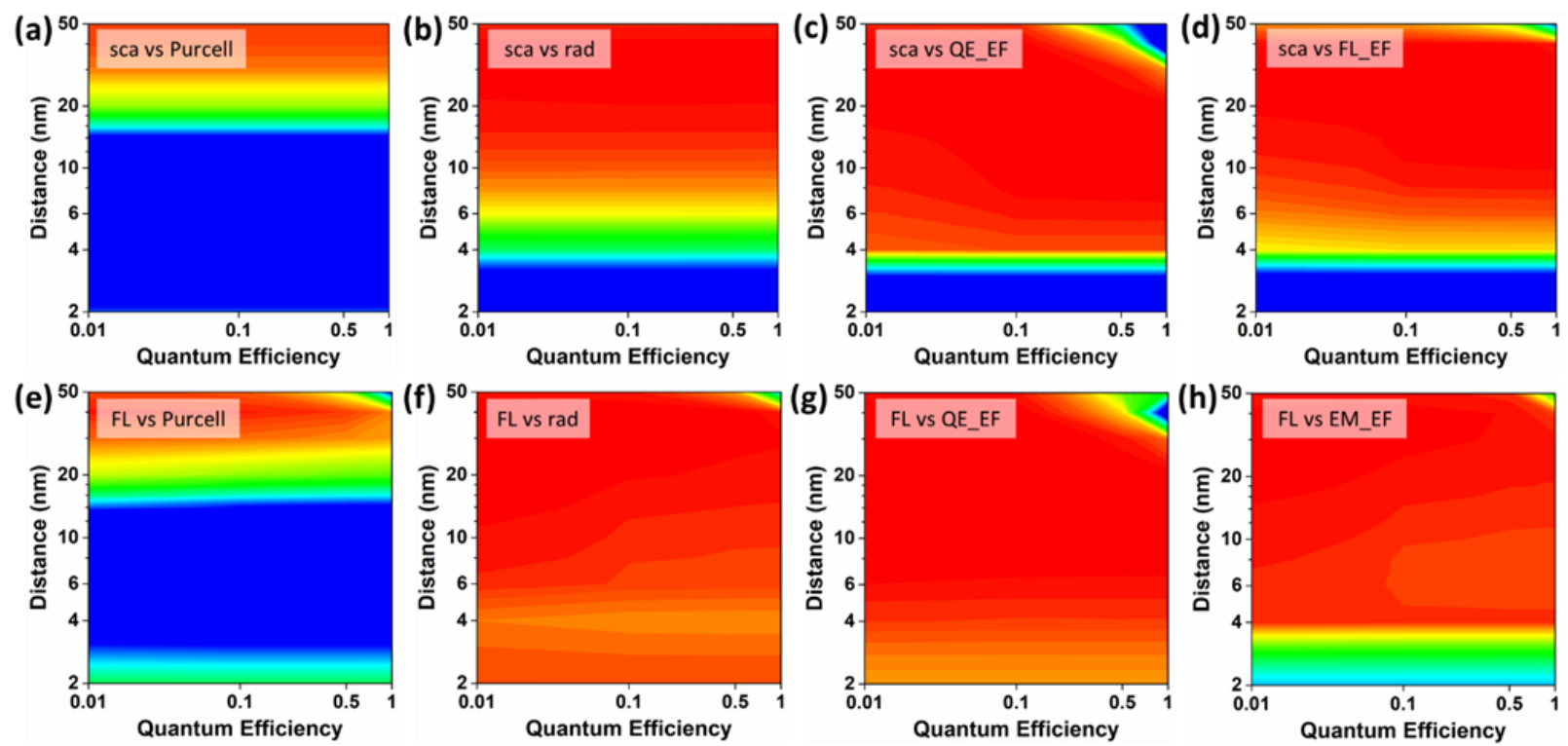

Figure 4.4 Correlation coefficients between scattering and (a) Purcell factor (b) radiative decay rate (c) quantum efficiency enhancement and (d) fluorescence enhancement; similarly, correlation coefficients between fluorescence enhancement and (e) Purcell factor (f) radiative decay rate ( $g$ ) quantum efficiency enhancement and (h) local EM field enhancement. The x-axis represents the intrinsic quantum efficiency. Log scale was applied to both the $x$ - and $y$-axis.

\section{Correlation Coefficients}

To further understand and clarify the relations among various parameters, correlation coefficients were calculated and presented in Figure 4.4 with respect to the intrinsic quantum efficiency and the separation distance between the quantum emitter and the Ag nanosphere. As expected, the intrinsic quantum efficiency did not play a role in the correlation relations. The separation distance not only modulated the excitation rate enhancement and quantum efficiency enhancement, but also defined three correlation regions, i.e. strongly-correlated region, weaklycorrelated region, and uncorrelated region. From figure 4.4(a) to (d), several observations can be 
made. Firstly, despite a strongly-correlated region observed at a separation distance larger than 30 $\mathrm{nm}$ in Figure 4.4(a), scattering did not meaningfully correlate with the Purcell factor owing to the weak fluorescence enhancement in this region, as shown in Figure 4.3(d). Secondly, scattering strongly correlated with radiative decay rate at a separation distance larger than $10 \mathrm{~nm}$ as shown in Figure 4.4(b), implying that scattering could be a tuning parameter in radiative decay engineering. Most importantly, scattering was found to strongly correlate with the fluorescence enhancement because of the strong correlation between scattering and quantum efficiency enhancement, provided that the quantum emitter was kept at least $5 \mathrm{~nm}$ away from the $\mathrm{Ag}$ nanosphere to avoid fluorescence quenching. Similarly, the correlation coefficients between the fluorescence enhancement and the Purcell factor, radiative decay rate, quantum efficiency enhancement, and local EM field enhancement were calculated, as shown in Figure 4.4(e) to (h). The strong correlation between the fluorescence enhancement and the quantum efficiency enhancement further underscored the role of scattering, which directly contributed to the quantum efficiency enhancement.

\subsubsection{Conclusion}

In summary, the effect of scattering on PEF was systematically studied by considering the interaction between a quantum emitter and an $\mathrm{Ag}$ nanosphere. It was found that the effect of scattering on PEF was a universal phenomenon regardless of the materials. More importantly, scattering was found to play dual roles in PEF. It not only modulates the local EM field enhancement, but also monotonically enhances the quantum efficiency. This study suggests that, in additional to the separation distance between the quantum emitter and the Ag nanosphere, scattering is also an important tuning parameter in PEF. 


\section{Reference}

1. Anger, P.; Bharadwaj, P.; Novotny, L., Enhancement and Quenching of Single-Molecule Fluorescence. Phys Rev Lett 2006, 96 (11), 113002.

2. $\quad$ Liu, X.; Yue, Q.; Yan, T.; Li, J.; Yan, W.; Ma, J.; Zhao, C.; Zhang, X., Competition between Local Field Enhancement and Nonradiative Resonant Energy Transfer in the Linear Absorption of a Semiconductor Quantum Dot Coupled to a Metal Nanoparticle. The Journal of Physical Chemistry C 2016, 120 (32), 18220-18227.

3. Nepal, D.; Drummy, L. F.; Biswas, S.; Park, K.; Vaia, R. A., Large Scale Solution Assembly of Quantum Dot-Gold Nanorod Architectures with Plasmon Enhanced Fluorescence. Acs Nano 2013, 7 (10), 9064-9074.

4. Ming, T.; Chen, H.; Jiang, R.; Li, Q.; Wang, J., Plasmon-Controlled Fluorescence: Beyond the Intensity Enhancement. The Journal of Physical Chemistry Letters 2012, 3 (2), 191202.

5. Lakowicz, J. R.; Ray, K.; Chowdhury, M.; Szmacinski, H.; Fu, Y.; Zhang, J.; Nowaczyk, K., Plasmon-controlled fluorescence: a new paradigm in fluorescence spectroscopy. Analyst 2008, 133 (10), 1308-1346.

6. Li, M.; Cushing, S. K.; Wu, N., Plasmon-enhanced optical sensors: a review. Analyst 2015, 140 (2), 386-406.

7. Liu, S.-Y.; Huang, L.; Li, J.-F.; Wang, C.; Li, Q.; Xu, H.-X.; Guo, H.-L.; Meng, Z.-M.; Shi, Z.; Li, Z.-Y., Simultaneous Excitation and Emission Enhancement of Fluorescence Assisted by Double Plasmon Modes of Gold Nanorods. The Journal of Physical Chemistry C 2013, 117 (20), 10636-10642.

8. Sun, G.; Khurgin, J. B.; Yang, C. C., Impact of high-order surface plasmon modes of metal nanoparticles on enhancement of optical emission. Appl Phys Lett 2009, 95 (17), 171103. 9. Khurgin, J. B.; Sun, G., Enhancement of optical properties of nanoscaled objects by metal nanoparticles. J. Opt. Soc. Am. B 2009, 26 (12), B83-B95.

10. Sun, G.; Khurgin, J. B., Origin of giant difference between fluorescence, resonance, and nonresonance Raman scattering enhancement by surface plasmons. Phys Rev A 2012, 85 (6), 063410 .

11. Abadeer, N. S.; Brennan, M. R.; Wilson, W. L.; Murphy, C. J., Distance and Plasmon Wavelength Dependent Fluorescence of Molecules Bound to Silica-Coated Gold Nanorods. Acs Nano 2014, 8 (8), 8392-8406.

12. Gandra, N.; Portz, C.; Tian, L.; Tang, R.; Xu, B.; Achilefu, S.; Singamaneni, S., Probing Distance-Dependent Plasmon-Enhanced Near-Infrared Fluorescence Using Polyelectrolyte Multilayers as Dielectric Spacers. Angewandte Chemie International Edition 2013, 53 (3), 866870.

13. Lu, G.; Zhang, T.; Li, W.; Hou, L.; Liu, J.; Gong, Q., Single-Molecule Spontaneous Emission in the Vicinity of an Individual Gold Nanorod. The Journal of Physical Chemistry C 2011, 115 (32), 15822-15828. 
14. Agio, M.; Cano, D. M., The Purcell factor of nanoresonators. Nature Photonics 2013, 7, 674.

15. Kinkhabwala, A.; Yu, Z.; Fan, S.; Avlasevich, Y.; Müllen, K.; Moerner, W. E., Large single-molecule fluorescence enhancements produced by a bowtie nanoantenna. Nature Photonics 2009, 3, 654.

16. Camposeo, A.; Persano, L.; Manco, R.; Wang, Y.; Del Carro, P.; Zhang, C.; Li, Z.-Y.; Pisignano, D.; Xia, Y., Metal-Enhanced Near-Infrared Fluorescence by Micropatterned Gold Nanocages. Acs Nano 2015, 9 (10), 10047-10054.

17. Bardhan, R.; Grady, N. K.; Cole, J. R.; Joshi, A.; Halas, N. J., Fluorescence Enhancement by Au Nanostructures: Nanoshells and Nanorods. Acs Nano 2009, 3 (3), 744-752.

18. Tam, F.; Goodrich, G. P.; Johnson, B. R.; Halas, N. J., Plasmonic Enhancement of Molecular Fluorescence. Nano Letters 2007, 7 (2), 496-501.

19. Song, J.-H.; Atay, T.; Shi, S.; Urabe, H.; Nurmikko, A. V., Large Enhancement of Fluorescence Efficiency from CdSe/ZnS Quantum Dots Induced by Resonant Coupling to Spatially Controlled Surface Plasmons. Nano Letters 2005, 5 (8), 1557-1561.

20. Szmacinski, H.; Lakowicz, J. R.; Catchmark, J. M.; Eid, K.; Erson, J. P.; Middendorf, L., Correlation between Scattering Properties of Silver Particle Arrays and Fluorescence Enhancement. Applied Spectroscopy 2008, 62 (7), 733-738.

21. Munechika, K.; Chen, Y.; Tillack, A. F.; Kulkarni, A. P.; Plante, I. J.-L.; Munro, A. M.; Ginger, D. S., Spectral Control of Plasmonic Emission Enhancement from Quantum Dots near Single Silver Nanoprisms. Nano Letters 2010, 10 (7), 2598-2603.

22. Saitow, K.-i.; Suemori, H.; Tamamitsu, H., Enhancement of fluorescence intensity by silicon particles and its size effect. Chem Commun 2014, 50 (9), 1137-1140.

23. Aslan, K.; Leonenko, Z.; Lakowicz, J. R.; Geddes, C. D., Annealed Silver-Island Films for Applications in Metal-Enhanced Fluorescence: Interpretation in Terms of Radiating Plasmons. Journal of Fluorescence 2005, 15 (5), 643.

24. Lakowicz, J. R., Radiative decay engineering 5: metal-enhanced fluorescence and plasmon emission. Analytical Biochemistry 2005, 337 (2), 171-194.

25. Liu, I. S.; Chen, Y.-F.; Su, W.-F., Modulating the photoluminescence of conducting polymer by the surface plasmon of Au colloids. Journal of Photochemistry and Photobiology A: Chemistry 2008, 199 (2), 291-296. 


\subsection{Near-Infrared Fluorescent Biosensor for Traumatic Brain Injury Biomarker Detection}

\subsubsection{Introduction}

Traumatic brain injury (TBI), primarily caused by falls and being struck by or against an object, accounts for about $30 \%$ of all injury deaths according to Centers for Disease Control and Prevention. ${ }^{1}$ While early treatment could effectively reduce short- and long-term adverse clinic outcomes, immediate diagnosis of TBI remains highly challenging owing to a lack of ultrasensitive point-of-care (POC) biosensor devices and the symptoms which may not appear until after days or even weeks. General guidelines currently widely in use for TBI screening, such as Glasgow Coma Scale, measurements for level of TBI, speech and language tests, and cognition and neuropsychological tests, proves ineffective, particularly for mild and moderate TBI or TBI at an early stage. ${ }^{2}$ Of more effective in screening, diagnosis, and prognosis are biomarkers, which are capable of revealing the full spectrum of biological disorders from initial manifestations to terminal stages. ${ }^{3-4}$ Currently widely acknowledged TBI biomarkers include glial fibrillary acidic protein (GFAP), S100- $\beta$, and neuron-specific enolase (NSE). ${ }^{5-6}$ However, it remains technically challenging to identify among complex biological environment a trace amount of the target and quantify it. Therefore, exploiting the full potential of TBI biomarkers falls on developing ultrasensitive POC biosensor devices which need also be compatible with the biological environment.

Typical methods for protein biomarker detection include enzyme-linked immunosorbent assay (ELISA), liquid chromatography-mass spectrometry (LC-MS), and Western blot. ${ }^{7-9}$ Despite their superb sensitivity with a limit of detection (LOD) even down to $\mathrm{pg} / \mathrm{mL}$ level for ELISA, it is difficult to transform them into POC biosensor devices, because they are either technically complex requiring significant professional expertise and sophisticated instrumentations or timeconsuming requiring tedious operational procedures. ${ }^{8}$ Recently, electrochemical methods have been implemented for TBI biomarkers detection given their strong performance as electrochemical biosensors, but most of them were only demonstrated in PBS buffer. ${ }^{10-15}$ They fall far short of the required compatibility with the biological environment while maintaining a high level of sensitivity.

Recently, fluorescent biosensors have received increasing attention. ${ }^{16-20}$ However, most of current fluorescent biosensors transduce visible fluorescent signal, which is not compatible with biological environment. Our studies have shown that the visible fluorescence intensity drops significantly with abnormally emission peak broadening in biological fluids. They are not suited to be extended for biomarkers detection in biological samples. As compared to visible fluorescence, 
NIR fluorescence only suffers a minimal interference in biological environment. ${ }^{21-23}$ This can be attributed to the unique features of the biological transparency windows, in which there is only low light absorbance and scattering by biological substances with significantly reduced autofluorescence. These unique features make NIR fluorescence especially advantageous over visible fluorescence in the biological environment. Furthermore, the availability of portable fluorescent readers in the market significantly stimulates research on NIR fluorescence-based POC biosensor devices. Nevertheless, NIR fluorophores usually exhibit a much less brightness as compared to the visible counterparts. ${ }^{24}$ Because their molecular energy levels are more likely to overlap with one another, there is a higher chance that non-radiative energy transfer could occur and end up significantly reducing the fluorescence intensity. Fortunately, the two-step process nature of fluorescence involving excitation and emission allows us to improve the brightness of NIR fluorophores by optimizing either the excitation, the emission, or both. ${ }^{25}$

Plasmon-enhanced fluorescence are currently actively explored to improve the fluorescence emission. There are two mechanisms for plasmon-enhanced fluorescence: excitation enhancement and emission enhancement. ${ }^{19,26-31}$ The excitation enhancement of plasmon-enhanced fluorescence is similar to that of surface-enhanced Raman scattering which scales with the square of the incident field. As the local field of the point dipole-like nanoparticle has $\frac{1}{d^{3}}$ dependence, the excitation enhancement would then have $\frac{1}{d^{6}}$ dependence. Therefore, the excitation enhancement is highly distance-dependent and decays rapidly when the separation distance between the fluorophore and the plasmonic nanostructure increases. In the emission process, the probability for the excited fluorophore to emit a photon depends on the competition between radiative and non-radiative decay, which is represented by the quantum efficiency. Based on Fermi's golden rule, the spontaneous decay rate scales with the local optical density of states. Therefore, the emission enhancement could result from the increased local optical density of states introduced by the plasmonic nanostructure. Unfortunately, while minimizing the mode volume in which the local electric field is confined could maximize the excitation enhancement, it also introduces strong energy loss channels which could significantly decrease the quantum efficiency and end up compromising the overall fluorescence intensity. This phenomenon is especially obvious on open plasmonic metallic nanostructures, where the excitation enhancement is predominately responsible for the fluorescence enhancement. ${ }^{32-34}$

In this study, a NIR fluorescent biosensor was developed for GFAP biomarker detection. The NIR fluorophore used is Dylight 755, which was labelled on the GFAP antibody. It has a peak excitation and emission wavelength of $755 \mathrm{~nm}$ and $775 \mathrm{~nm}$, respectively, with an intrinsic quantum efficiency of $11.9 \%,{ }^{35}$ which is among the highest in NIR fluorophores. A two-dimensional (2D) Au nanopyramid array pattern was fabricated on glass by nanosphere lithography ${ }^{36-37}$ and used as the biosensing platform after being functionalized with the GFAP capture antibody. As a proof-ofconcept, the NIR fluorescent biosensor has been demonstrated to detect GFAP biomarker in human 
blood plasma with a LOD of $0.6 \mathrm{pg} / \mathrm{mL}$. Notably, a portable POC fluorescence reader was used to conduct all the tests instead of the sophisticated spectrofluorometer. Finite-difference time-domain (FDTD) simulations were also implemented to identify the origin of the fluorescence enhancement as well as to calculate the enhancement factor. It was found that the observed fluorescence enhancement was primarily caused by a huge excitation enhancement due to the intense local electric field supported on the sharp corners and edges of the Au nanopyramid array substrate.

\section{(a) Glass Cleaning}

(b) Polystyrene Beads Assembly

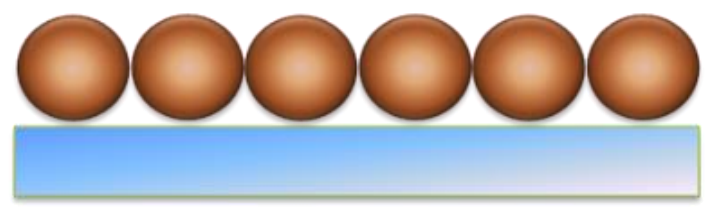

(c) Titanium Deposition

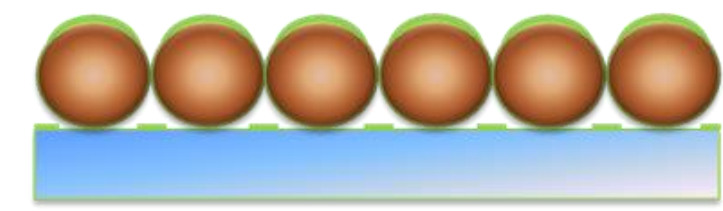

(d) Gold Deposition

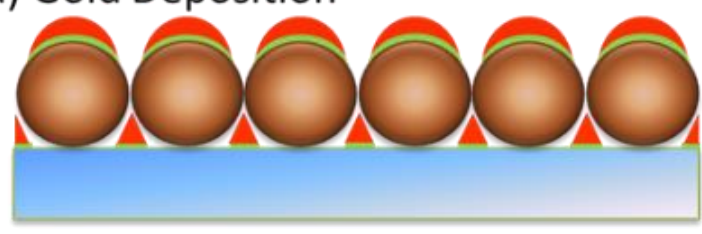

(f) Removal of Polystyrene Beads

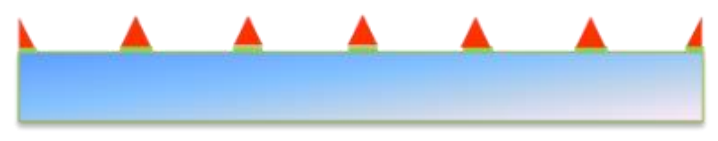

(h) Schematic Gold Nanopyramid Array

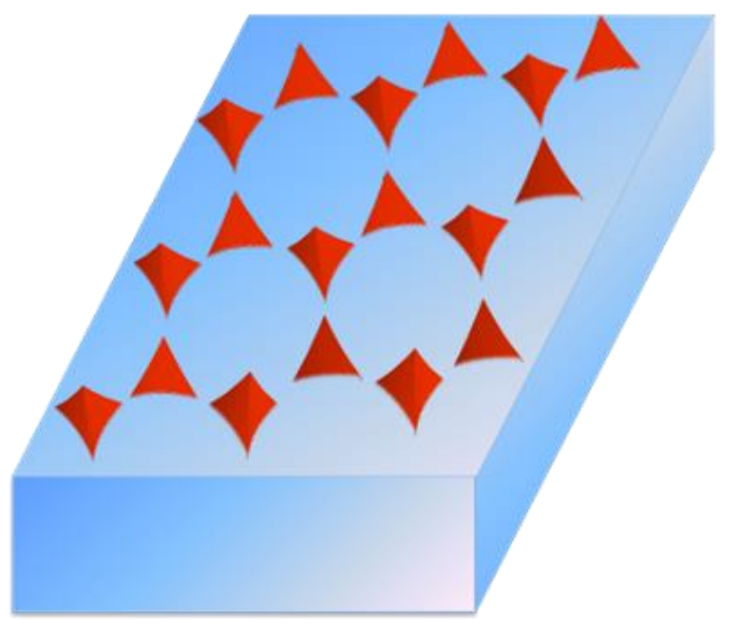

Figure 4.5 Nanosphere lithography fabrication of gold nanopyramid array on glass substrate. Polystyrene beads with a diameter of $500 \mathrm{~nm}$ were used. $5 \mathrm{~nm}$ of titanium and $250 \mathrm{~nm}$ of gold were deposited.

\subsubsection{Methods}

\section{Chemicals and Materials}

(3-Mercaptopropyl) trimethoxysilane (95\%), BSA (bovine serum albumin), phosphate buffered saline tablets, monoclonal anti-glial fibrillary acidic protein antibody produced in mouse (G3893-.2ML), glial fibrillary acidic protein human brain (345996-100UG), goat anti-human IgG polyclonal antibody, mice anti-goat IgG polyclonal antibody, IgG from human serum, sodium silicate solution (338443-1L), N-hydroxysuccinimide (NHS), and 1-ethyl-3-(3-(dimethylamino)propyl) carbodiimide (EDC), were purchased from Sigma-Aldrich. The IgG-free blood plasma was purchased from US Biological Life Science (P4252-56 Plasma, Human). Dylight 755 NHS 
Ester Microscale Antibody Labelling Kit and polystyrene microspheres with a diameter of $600 \mathrm{~nm}$ were purchased from Thermo Scientific. DI water used for washing and reactions was produced by Milli-Q Millipore system (18.2 M $\mathrm{cm}$, Millipore Corp., Billerica, MA). All chemicals obtained from commercial vendors and were directly used without further purification.
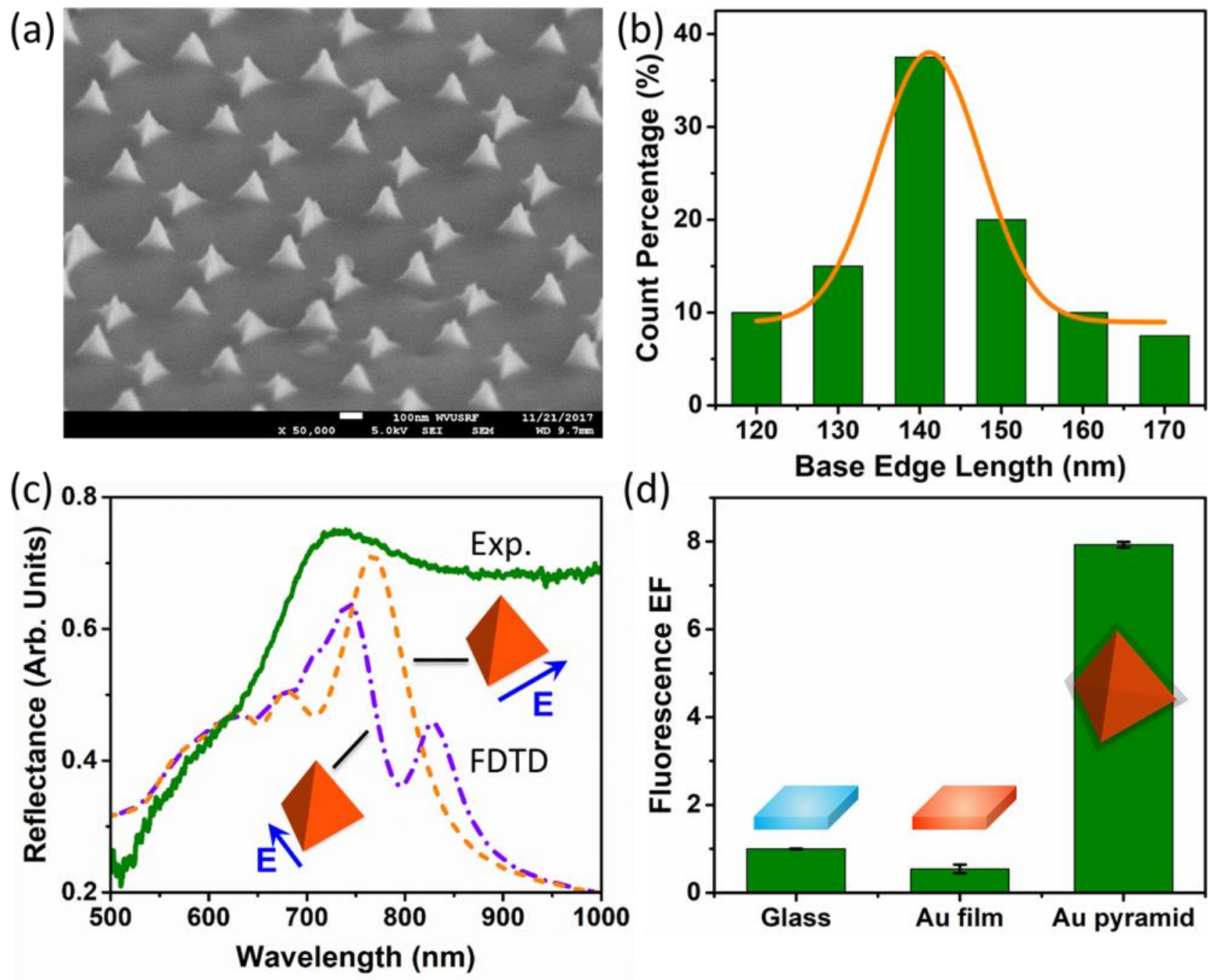

Figure 4.6 (a) SEM image of the as-fabricated Au nanopyramid arrays; (b) Statistic analysis of the pyramid base edge length, which was found to be $141 \mathrm{~nm}$ based on Gaussian fitting; (c) Comparison of the experimentally measured (solid olive curve) and FDTD-simulated (dash and dash dotted curves corresponding to two orthogonal polarizations shown as the blue arrows) reflection spectra with an input plane wave incident from the top; (d) Comparison of the fluorescence intensity of Dylight 755 with a concentration of $1 \mu \mathrm{g} / \mathrm{mL}$ on glass, Au film, and Au nanopyramid arrays coated with a layer of $2.4 \mathrm{~nm}$ silica.

\section{Gold Nanopyramid Array Fabrication}

$\mathrm{Au}$ nanopyramid arrays were fabricated on glass slides (roughly $0.5 \mathrm{~cm} \times 0.5 \mathrm{~cm}$ ) by nanosphere lithography in the cleanroom, ${ }^{38}$ as shown in Figure 4.5. Prior to the fabrication, glass slides were cleaned by using acid piranha (the ratio of sulfuric acid to hydrogen peroxide is $3: 1$ ) at $90{ }^{\circ} \mathrm{C}$ for 1 hour. Caution: acid piranha is very dangerous and needs to be handled with extreme 
care! They were subsequently rinsed with DI water, cleaned by ultrasonication three times in ethanol and DI water, respectively. After that, a monolayer of PS beads was dip-coated on glass slides, which formed a hexagonal pattern. Afterwards, a $5 \mathrm{~nm}$ thick titanium layer followed by a $250 \mathrm{~nm}$ thick gold layer were deposited by e-beam evaporator. After removal of the PS beads by ultrasonication in methanol and being dried using compressed air, hexagonally ordered $\mathrm{Au}$ nanopyramid arrays were obtained, as shown in Figure 4.6.

\section{Thin Silica Coating of Gold Nanopyramid Array}

Following the protocol reported before, ${ }^{39}$ a thin layer of silica was conformationally grown on $\mathrm{Au}$ nanopyramid arrays. Specifically, the as-fabricated $\mathrm{Au}$ nanopyramid arrays were first incubated in the ethanolic solution of (3-Mercaptopropyl) trimethoxysilane with a concentration of $2 \mathrm{mM}$ for 2 hours. After thorough DI water rinsing, those Au nanopyramid arrays were then incubated in sodium silicate solution (1.5 wt\%, pH 8 9) at $90{ }^{\circ} \mathrm{C}$ for 2 days. In the meanwhile, $\mathrm{Au}$ film substrates as control samples were also coated with a thin layer of silica under the same conditions. Since it is very difficult to directly measure the actual thickness of silica grown on Au nanopyramid arrays, we assume that the silica grown on Au nanopyramid arrays has a similar thickness as the one grown on $\mathrm{Au}$ film, which can be characterized by ellipsometry.

\section{Dylight 755 Antibody Labelling}

Following the protocol provided by Thermo Scientific, the detection antibody was initially labelled with Dylight 755. Specifically, the concentration of detection antibody was adjusted to 1 $\mathrm{mg} / \mathrm{mL}$ in PBS buffer. Then $8 \mu \mathrm{L}$ of Borate Buffer $(0.67 \mathrm{M})$ was added to $100 \mu \mathrm{L}$ of $1 \mathrm{mg} / \mathrm{mL}$ antibody in PBS. The mixed solution was further added to the Dylight Reagent vial containing 15 $\mu \mathrm{g}$ Dylight 755 NHS ester power. After thorough mixing, the solution was incubated at room temperature for $1 \mathrm{~h}$ protected from light. To purify the Dylight-labelled antibody, the above solution was added to the purification resin and then centrifuged for 1 minute at $1000 \times \mathrm{g}$. The obtained Dylight-labelled was stored at $4{ }^{\circ} \mathrm{C}$ in dark for future use.

\section{Operating Principle of Biosensor}

Initially, the detection antibody was labelled with Dylight 755; in the meanwhile, Au nanopyramid arrays were coated with a thin layer of silica and then functionalized with the capture antibody. To realize biosensing, capture antibody-functionalized Au nanopyramid arrays were first incubated into the analyte solution for $30 \mathrm{~min}$. After thorough rinsing with PBS buffer, these Au nanopyramid arrays were then incubated into the Dylight-labelled detection antibody solution for another $30 \mathrm{~min}$. After thorough rinsing with PBS buffer and dried using compressed air, the Au nanopyramid arrays were characterized by a portable POC fluorescence reader. The schematic operation principle was shown in Figure 4.7. 


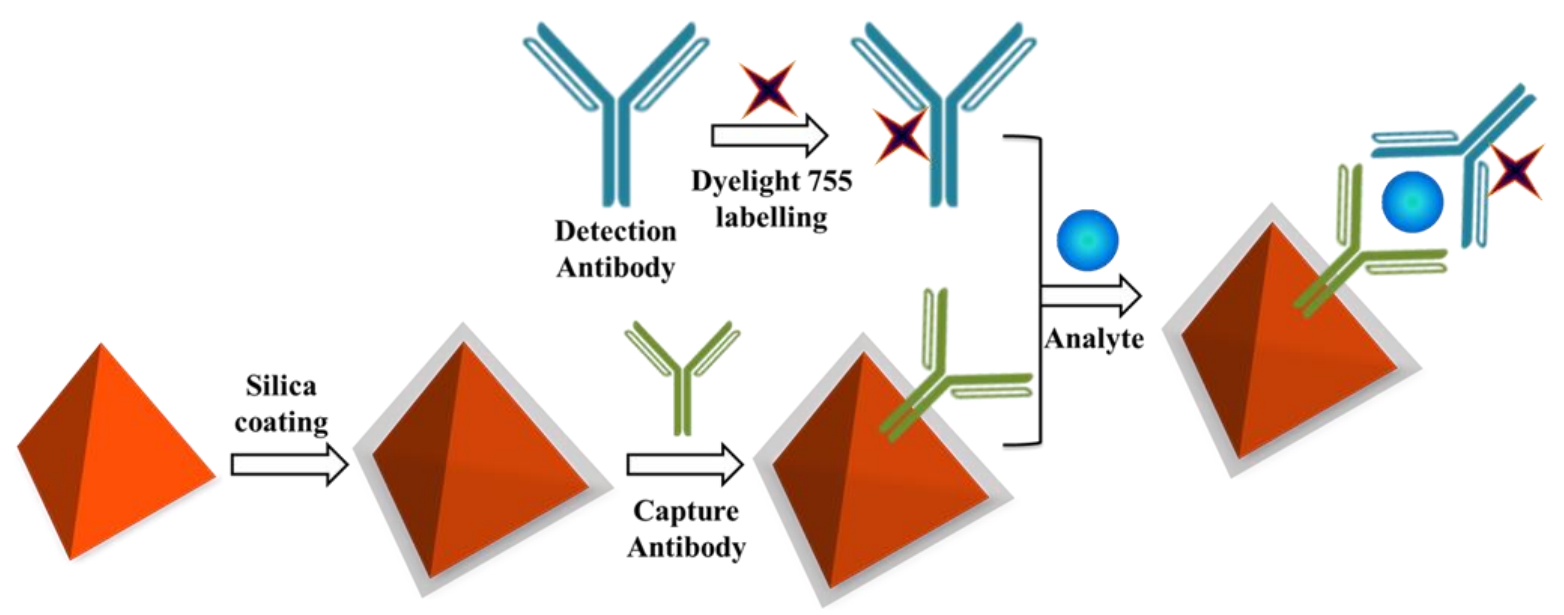

Figure 4.7 Operating principle of the plasmonic fluorescent biosensor

\section{IgG Detection in PBS Buffer}

IgG with a series of concentrations of $1 \mathrm{pg} / \mathrm{mL}, 10 \mathrm{pg} / \mathrm{mL}, 100 \mathrm{pg} / \mathrm{mL}, 1 \mathrm{ng} / \mathrm{mL} 10 \mathrm{ng} / \mathrm{mL}$, $100 \mathrm{ng} / \mathrm{mL}, 1 \mu \mathrm{g} / \mathrm{mL} 10 \mu \mathrm{g} / \mathrm{mL}$, and $100 \mu \mathrm{g} / \mathrm{mL}$ were prepared in PBS buffer. Detection $\operatorname{IgG}$ antibody was labelled with Dylight 755 following the above procedure; capture IgG antibody was immobilized on the silica-coated Au nanopyramid arrays. Following the above operation principle and the scheme in Figure 4.7, IgG detection was realized using the plasmonic fluorescent biosensor.

\section{GFAP Detection in Blood Plasma}

GFAP with a series of concentrations of $1 \mathrm{pg} / \mathrm{mL} 2 \mathrm{pg} / \mathrm{mL}, 5 \mathrm{pg} / \mathrm{mL}, 10 \mathrm{pg} / \mathrm{mL}, 20 \mathrm{pg} / \mathrm{mL}$, $50 \mathrm{pg} / \mathrm{mL}, 100 \mathrm{pg} / \mathrm{mL}, 200 \mathrm{pg} / \mathrm{mL} 500 \mathrm{pg} / \mathrm{mL}, 1 \mathrm{ng} / \mathrm{mL}, 2 \mathrm{ng} / \mathrm{mL}, 5 \mathrm{ng} / \mathrm{mL}, 10 \mathrm{ng} / \mathrm{mL}, 20 \mathrm{ng} / \mathrm{mL}$, $50 \mathrm{ng} / \mathrm{mL}$ and $100 \mathrm{ng} / \mathrm{mL}$ were prepared in blood plasma. Detection GFAP antibody was labelled with Dylight 755 following the above procedure; capture GFAP antibody was immobilized on the silica-coated Au nanopyramid arrays. Following the similar procedure for IgG detection, GFAP detection in blood plasma was realized.

\section{Instruments and Characterizations}

Titanium and gold layers were deposited by an e-beam evaporator (Kurt J Lesker, Model\#LAB18). The thin silica layer grown on Au film was characterized by ellipsometry (J.A. Woollam M-2000U Ellipsometer), which gave a mean thickness of $2.4 \mathrm{~nm}$ from 3 independent samples. Au nanopyramid arrays were imaged using a JEOL JSM-7600F scanning electron microscope (SEM). An Ocean Optics USB 4000 spectrometer was used to take the reflection spectra of Au nanopyramid arrays. A compact point-of-care (cPOC) near-infrared fluorescence reader (cPoCLabFluo v2.3.0, LRE Medical GmbH, München, Germany) was used to read the fluorescence intensity of the biosensor; it has an excitation wavelength of $755 \mathrm{~nm}$ and collects fluorescence emission at a wavelength of $775 \mathrm{~nm}$. 


\section{Finite-Difference Time-Domain (FDTD) Simulations}

The software Lumerical FDTD Solutions was implemented to calculate the optical properties of $\mathrm{Au}$ nanopyramid arrays, the electric field enhancement, and the quantum efficiency enhancement. A mesh size of $2 \mathrm{~nm}$ was used in all simulations. The permittivity of gold used was from Johnson \& Christy. ${ }^{40}$ The refractive index was set as 1.52 for the glass substrate. A plane wave was used as the input light source. Perfectly Matched Layer boundary conditions were imposed. An Au nanopyramid on glass was modelled to have a base edge length of $140 \mathrm{~nm}$ and a height of $250 \mathrm{~nm}$ in this work.

\subsubsection{Results and Discussions}

\section{Physical and Optical Properties of Gold Nanopyramid Arrays}

Nanosphere lithography was implemented to fabricate $\mathrm{Au}$ nanopyramid arrays on glass substrates following a similar protocol we reported before, ${ }^{38}$ which can be found in Figure 4.5. The as-fabricated Au nanopyramids were arranged hexagonally with a base edge length of $141 \mathrm{~nm}$, as shown in Figure 4.6(a) and (b). The plasmonic corner modes was spectrally visible and exhibited as a reflection peak at $735 \mathrm{~nm}$, as shown in Figure 4.6(c). FDTD method was implemented to calculate the reflection spectra for an Au nanopyramid with a height of $250 \mathrm{~nm}$ and a base edge length of $140 \mathrm{~nm}$ on glass. Two orthogonal polarizations were considered, and the calculated reflection spectra were shown in Figure 4.6(c). The overall spectral line shapes were consistent with the experimentally measured one. The longest wavelength peaks were from the plasmonic corner modes while the second longest from the edge modes and other higher-order modes. It is noted that a lack of these fine features on the experimentally measured reflection spectrum can be reasonably attributed to the rounded corners and edges as well as random orientations for the fabricated Au nanopyramid arrays on a large area.

To assess the performance of $\mathrm{Au}$ nanopyramid arrays in enhancing fluorescence, $10 \mu \mathrm{L}$ of 1 $\mu \mathrm{g} / \mathrm{mL}$ Dylight 755 was drop-coated on a glass, an Au film, and an Au nanopyramid array substrate coated with a layer of $\sim 2.4 \mathrm{~nm}$ silica. The purpose of coating a thin layer of silica on the $\mathrm{Au}$ nanopyramid array was to minimize fluorescence quenching due to direct contact between the metallic nanostructure and the fluorophore. Figure 4.6(d) shows the fluorescence enhancement factors normalized by the glass substrate. The fluorescence enhancement factors were found to be 0.5 and 8 for $\mathrm{Au}$ film and silica-coated Au nanopyramid array, respectively. It indicates that the fluorescence got partially quenched on Au film but benefited 8 times enhancement on silica-coated Au nanopyramid array. Therefore, Au nanopyramid array substrates proved effective in amplifying the fluorescence intensity of NIR fluorophores.

\section{Assessing Performance of the Plasmonic Fluorescent Biosensor}

Prior to TBI biomarker detection, the plasmonic fluorescent biosensor was implemented to detect IgG in PBS buffer to assess its performance and identify the linear working concentration 
range. In the first place, the IgG detection antibody was labelled with Dylight 755; the IgG capture antibody was immobilized on the silica-coated Au nanopyramid array, following the protocol in Figure 4.7. The presence of IgG allows the Dylight-labelled detection antibody to be captured onto the Au nanopyramid arrays. The measured fluorescence intensity from the Au nanopyramid arrays was found to increase with an increase of the added concentration of IgG till saturation. The linear working concentration range was identified to be from $1 \mathrm{pg} / \mathrm{mL}$ to $1 \mathrm{ng} / \mathrm{mL}$ in PBS buffer, as shown in Figure 4.8. In comparison, if built on a glass substrate, the fluorescent biosensor exhibited a much lower intensity and was unable to transduce a fluorescent signal until the concentration of IgG reached $1 \mathrm{ng} / \mathrm{mL}$.

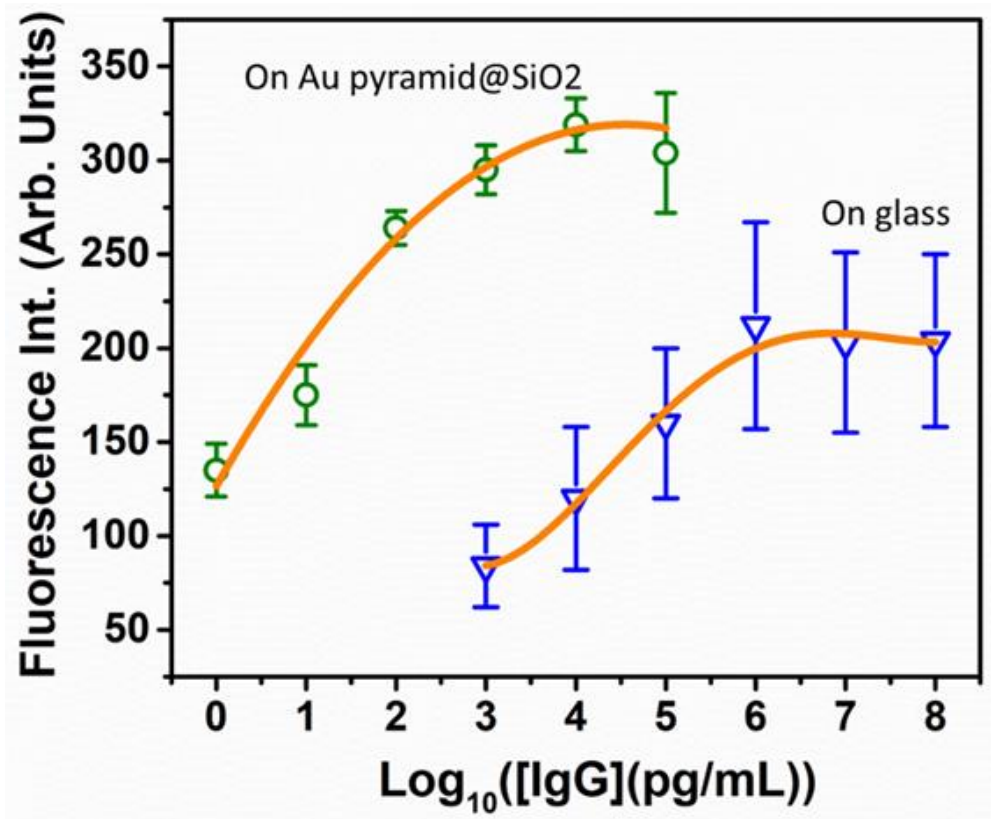

Figure 4.8 Comparison of IgG detection in PBS buffer on Au nanopyramid arrays and on glass. It indicates that the linear working range of the plasmonic fluorescent biosensor is roughly from $1 \mathrm{pg} / \mathrm{mL}$ to $1 \mathrm{ng} / \mathrm{mL}$.

\section{TBI Biomarker Detection in Blood Plasma}

The protocol for GFAP biomarker detection was similar to the one for IgG detection in PBS buffer, but GFAP antibodies were used instead of IgG antibodies. The protocols for synthesis of Dylight-labelled GFAP detection antibody and detection of GFAP in blood plasma can be found in the Methods Section. Various concentrations of GFAP in blood plasma were tested using the plasmonic fluorescent biosensor, as shown in Figure 4.9(a). The glass substrate was used as a control under the same conditions. The plasmonic fluorescent biosensor transduced a fluorescent signal even when the GFAP was just $1 \mathrm{pg} / \mathrm{mL}$ in blood plasma; in contrast, the control on glass substrate did not respond until the GFAP hit $1 \mathrm{ng} / \mathrm{mL}$. The fluorescent intensities for both increased with the GFAP concentration. However, the plasmonic fluorescent biosensor not only displayed a wider linear working concentration range from $1 \mathrm{pg} / \mathrm{mL}$ to $500 \mathrm{pg} / \mathrm{mL}$, but also responded to lower concentrations of GFAP, as compared to the case on glass substrate shown in Figure 4.9(b). The 
linear working concentration range for the plasmonic fluorescent biosensor was fitted with an equation of $y=127 x+147, R^{2}=97 \%$ with a limit of detection of $0.6 \mathrm{pg} / \mathrm{mL}$ toward GFAP biomarker in blood plasma, which was estimated based on three times signal-to-ratio.
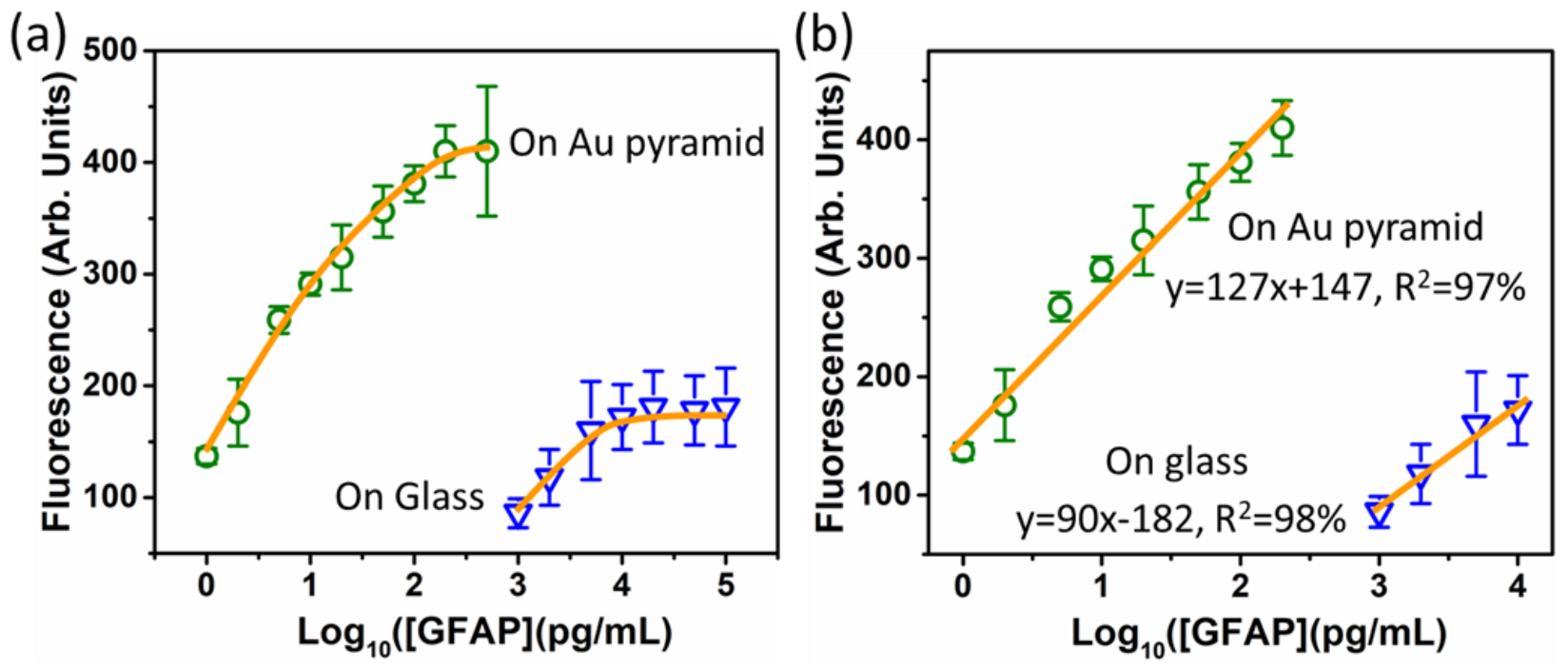

Figure 4.9 Detection of Glial Fibrillary Acidic Protein (GFAP) biomarker in blood plasma using based on Au nanopyramid arrays. (a) Performance comparison of the biosensor towards the detection of GFAP in blood plasma on the silica-coated Au nanopyramid arrays and on glass; (b) The linear working range extracted from (a).

Compared to other immunoassays for GFAP detection, our plasmonic fluorescent biosensor displayed a lower LOD, as compared in Table 4.1, which makes it more sensitive to conduct analysis of trace amount of biomarkers. It is suitable to work in blood plasma with little interference thanks to the NIR fluorescence in the first biological transparency window. Furthermore, use of the portable POC fluorescence reader to conduct all the tests helps significantly simplify the detection process. In addition, the GFAP cutoff concentration, which was reported to be in the range of 30-290 $\mathrm{pg} / \mathrm{mL},{ }^{41-44}$ also falls within the linear working concentration range of our plasmonic fluorescent biosensor. In summary, the plasmonic fluorescent biosensor demonstrated a strong capability for GFAP biomarker detection in human blood plasma. 
Table 4.1 A summary of recent work on GFAP detection.

\begin{tabular}{|c|c|c|c|c|c|}
\hline Methods & Transducer & Solvent & Detection Range & LOD & Source \\
\hline $\begin{array}{l}\text { GFAP-imprinted } \\
\text { polymer }\end{array}$ & Electrochemical & $\begin{array}{l}\mathrm{KCl} \text { and } \\
\text { ferrocyanide }\end{array}$ & $0.2-10 \mu \mathrm{g} / \mathrm{ml}$ & $0.04 \mu \mathrm{g} / \mathrm{mL}$ & Ref. $^{12}$ \\
\hline $\begin{array}{c}\text { Molecularly } \\
\text { imprinted polymer }\end{array}$ & Electrochemical & PBS buffer & $50-500 \mathrm{ng} / \mathrm{mL}$ & $17 \mathrm{ng} / \mathrm{mL}$ & Ref. ${ }^{10}$ \\
\hline Immunoassay & Colorimetric & PBS buffer & $1-100 \mathrm{ng} / \mathrm{mL}$ & $10 \mathrm{ng} / \mathrm{mL}$ & Ref..$^{45}$ \\
\hline Immunoassay & Fluorescence & Human serum & $0.1-8 \mathrm{ng} / \mathrm{mL}$ & $25 \mathrm{pg} / \mathrm{mL}$ & Ref. ${ }^{46}$ \\
\hline Immunoassay & Electrochemical & PBS buffer & $1 \mathrm{pg} / \mathrm{mL}-100 \mathrm{ng} / \mathrm{mL}$ & $1 \mathrm{pg} / \mathrm{mL}$ & Ref. $^{11}$ \\
\hline Immunoassay & Electrochemical & PBS buffer & $0.8-400 \mathrm{ng} / \mathrm{mL}$ & $1 \mathrm{ng} / \mathrm{mL}$ & Ref. $^{13}$ \\
\hline Immunoassay & Electrochemical & Human serum & $1 \mathrm{pg} / \mathrm{mL}-100 \mathrm{ng} / \mathrm{mL}$ & $1 \mathrm{pg} / \mathrm{mL}$ & Ref. $^{47}$ \\
\hline Immunoassay & Electrochemical & PBS buffer & $0.5-100 \mathrm{ng} / \mathrm{mL}$ & $1 \mathrm{ng} / \mathrm{mL}$ & Ref. ${ }^{15}$ \\
\hline Immunoassay & Electrochemical & PBS buffer & $2.9 \mathrm{pg} / \mathrm{mL}-2.9 \mu \mathrm{g} / \mathrm{mL}$ & $2.9 \mathrm{pg} / \mathrm{mL}$ & Ref. ${ }^{14}$ \\
\hline Immunoassay & Fluorescence & Human plasma & $1-500 \mathrm{pg} / \mathrm{mL}$ & $0.6 \mathrm{pg} / \mathrm{mL}$ & This work \\
\hline
\end{tabular}

\section{FDTD Calculation of Fluorescence Enhancement Factor}

To elucidate the origin of the fluorescence enhancement on Au nanopyramid arrays, FDTD calculations were implemented. The fluorophore was modeled as a classic point dipole and represented by a two-level system with a transition dipole moment of $\vec{p}$. For weak excitation, the fluorescence is a two-step process involving excitation and emission. Plasmon-enhanced fluorescence usually consists of both excitation rate enhancement $\left(\gamma_{\text {ext }} / \gamma_{\text {ext }}^{0}\right)$ and emission enhancement. The excitation enhancement is primarily caused by the huge amplification of the local field the fluorophore experiences and scales with the local electric field enhancement, as represented by:

$$
\frac{\gamma_{\text {ext }}}{\gamma_{\text {ext }}^{0}}=\left|\frac{\vec{p} \cdot \vec{E}}{\vec{p} \cdot \overrightarrow{E_{0}}}\right|^{2}
$$

where $\overrightarrow{E_{0}}$ and $\vec{E}$ are the input electric field in vacuum and the total electric field the fluorophore experiences in the presence of the Au nanopyramid. Maximum excitation enhancement can be obtained when the transition dipole is aligned with the electric field, and this scenario was considered in calculation of the excitation enhancement of Au nanopyramid arrays. 
The emission enhancement basically quantifies the modification of the quantum efficiency of the fluorophore due to the change of the local optical density of states under the influence of the Au nanopyramid. In the absence of the Au nanopyramid, the intrinsic quantum efficiency $\left(\eta_{0}\right)$ is given by:

$$
\eta_{0}=\frac{\gamma_{r}^{0}}{\gamma_{r}^{0}+\gamma_{n r}^{0}}
$$

where $\gamma_{r}^{0}$ and $\gamma_{n r}^{0}$ are the intrinsic radiative and non-radiative decay rate. The presence of the $\mathrm{Au}$ nanopyramid modified the quantum efficiency to $\eta$ because additional non-radiative decay rate $r_{a b s}$ was introduced, which could be caused by Ohmic losses in the system. The new quantum efficiency can be represented by:

$$
\eta=\frac{\gamma_{r}}{\gamma_{r}+r_{a b s}+r_{n r}}
$$

where $\gamma_{r}$ and $\gamma_{n r}$ are the new radiative and intrinsic non-radiative decay rate under the influence of Au nanopyramid. Suppose the intrinsic non-radiative rate remains unchanged, i.e. $r_{n r}=\gamma_{n r}^{0}$, the quantum efficiency enhancement can be evaluated by incorporating Equations (4.2) and (4.3), which is given by: ${ }^{48}$

$$
\frac{\eta}{\eta_{0}}=\frac{\frac{\gamma_{r}}{\gamma_{r}^{0}}}{\frac{\gamma_{r}}{\gamma_{r}^{0}} \eta_{0}+\frac{\gamma_{a b s}}{\gamma_{r}^{0}} \eta_{0}+\left(1-\eta_{0}\right)}
$$

where $\gamma_{r} / \gamma_{r}^{0}$ is the normalized radiative decay rate and can be obtained from the power monitor groups around the dipole in FDTD simulation software, $\gamma_{a b s} / \gamma_{r}^{0}$ is the normalized non-radiative decay rate due to Ohmic losses and can be obtained from the power monitor groups around the dipole-Au nanopyramid system. Therefore, the fluorescence enhancement factor (EF) containing both components and can be evaluated as:

$$
E F=\frac{\gamma_{\text {ext }}}{\gamma_{\text {ext }}^{0}} \frac{\eta}{\eta_{0}}
$$

In our study, the near-infrared fluorophore used is Dylight 755 , which has an intrinsic quantum efficiency of $11.9 \%$, among the highest of near-infrared fluorophores. In calculating the excitation enhancement, two orthogonal polarizations were considered for the input light source; the transition dipole moment of Dylight 755 perpendicular and parallel to the surface of $\mathrm{Au}$ nanopyramid were both considered and averaged to give the quantum efficiency enhancement. A total of 121 points on the plane $10 \mathrm{~nm}$ away from the surface of the Au nanopyramid coated with a $2 \mathrm{~nm}$ silica were calculated to map the excitation enhancement, the emission enhancement, and the fluorescence enhancement. Given the size of the antibody and antigen as well as the silica layer, 
calculation of planes $10 \mathrm{~nm}$ away from the surface of the Au nanopyramid was a reasonable choice and well justified.

(a)

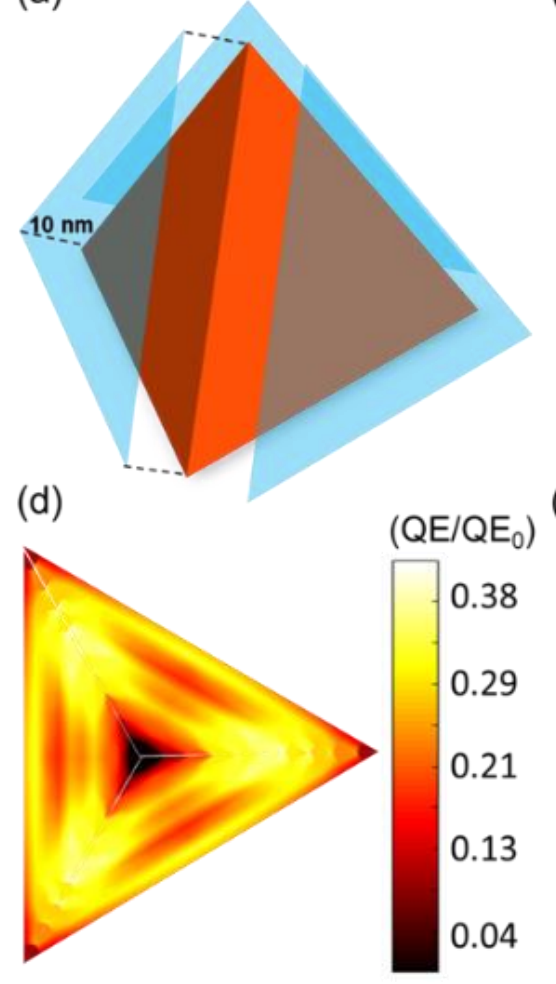

(b)

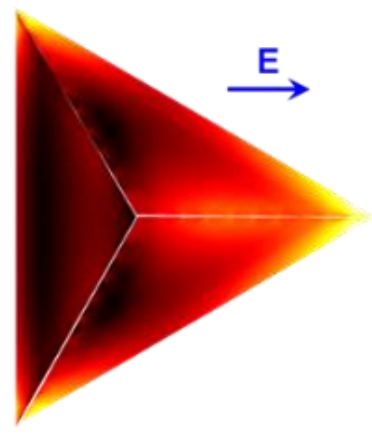

(e)

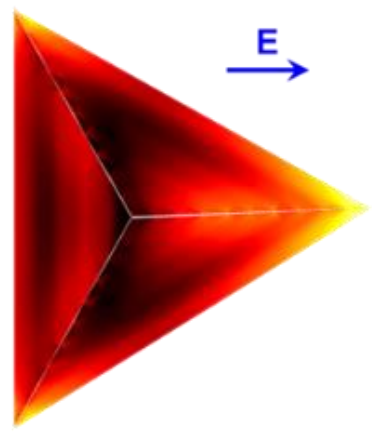

(c)

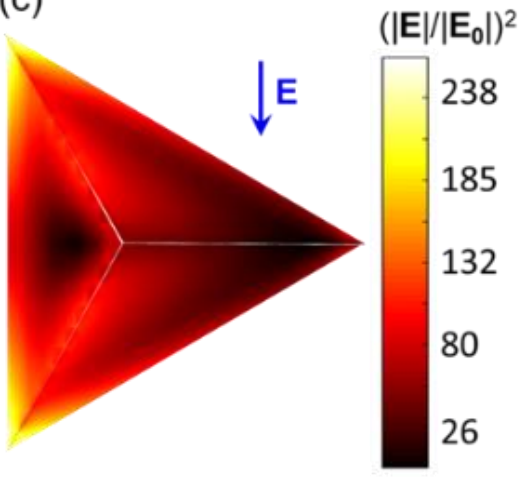

(f)

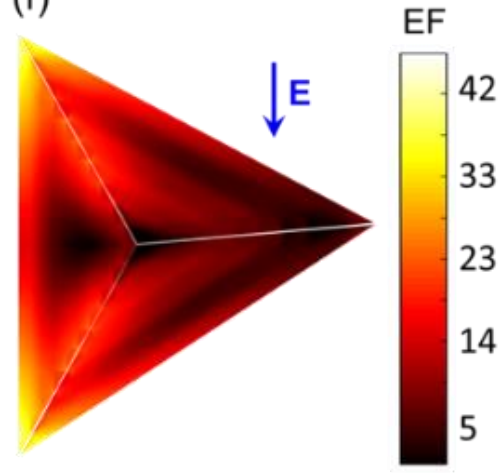

Figure 4.10 Calculation of fluorescence enhancement factors. (a) Scheme of the three planes (blue, each being $10 \mathrm{~nm}$ away from the surface of Au nanopyramid) on which the local electric field enhancement, quantum efficiency enhancement, and fluorescence enhancement were calculated; (b) and (c) Local electric field enhancement under orthogonal polarizations shown as blue arrows; (d) quantum efficiency enhancement averaged from the dipole perpendicular and parallel to the surface of Au nanopyramid array; (e) and (f) fluorescence enhancement factors under orthogonal polarizations shown as blue arrows. Light was incident from the top.

The calculated results were shown in Figure 4.10. Large excitation enhancement was found near and around the pyramid corner area. Notably, the quantum efficiency was reduced and minimized where the local electric field was maximized. This is because strong energy loss occurs when the electric field is tightly confined and thus contributes significantly to non-radiative decay. Nevertheless, the intense local electric field enhancement effectively compensated the quantum efficiency reduction and ended up enhancing the fluorescence across the entire nanostructure. A maximum fluorescence enhancement factor of 45 was achieved. In the meanwhile, large fluorescence enhancement factors could be found near and around the pyramid corner area. This 
indicated that the excitation enhancement was primarily responsible for the fluorescence enhancement on Au nanopyramid arrays.

\subsubsection{Conclusion}

In summary, a plasmonic fluorescent biosensor was developed and has been demonstrated for GFAP biomarker detection in human blood plasma. Compared to other immunosensors for GFAP detection, this plasmonic fluorescent biosensor exhibited a lower LOD and strong compatibility with the biological environment due to the use of NIR fluorescence in the first biological transparent window. Notably, a portable POC fluorescence reader was used to conduct the test instead of the sophisticated spectrofluorometer. FDTD simulations were also implemented and identified that the excitation enhancement from the corners and edges of the Au nanopyramid was primarily responsible for the fluorescence enhancement. 


\section{Reference}

1. Taylor, C. A.; Bell, J. M.; Breiding, M. J.; Xu, L. K., Traumatic Brain Injury-Related Emergency Department Visits, Hospitalizations, and Deaths - United States, 2007 and 2013. Mmwr. Surveill. Summ. 2017, 66 (9), 1-18.

2. Duhaime, A.-C.; Beckwith, J. G.; Maerlender, A. C.; McAllister, T. W.; Crisco, J. J.; Duma, S. M.; Brolinson, P. G.; Rowson, S.; Flashman, L. A.; Chu, J. J.; Greenwald, R. M., Spectrum of acute clinical characteristics of diagnosed concussions in college athletes wearing instrumented helmets. Journal of Neurosurgery 2012, 117 (6), 1092-1099.

3. Mayeux, R., Biomarkers: Potential uses and limitations. NeuroRX 2004, 1 (2), 182-188.

4. Strimbu, K.; Tavel, J. A., What are biomarkers? Current Opinion in HIV and AIDS 2010, $5(6), 463-466$.

5. Pelinka, L. E.; Kroepfl, A.; Leixnering, M.; Buchinger, W.; Raabe, A.; Redl, H., GFAP Versus S100B in Serum after Traumatic Brain Injury: Relationship to Brain Damage and Outcome. Journal of Neurotrauma 2004, 21 (11), 1553-1561.

6. Zetterberg, H.; Smith, D. H.; Blennow, K., Biomarkers of mild traumatic brain injury in cerebrospinal fluid and blood. Nature Reviews Neurology 2013, 9, 201.

7. Lilja, H.; Ulmert, D.; Vickers, A. J., Prostate-specific antigen and prostate cancer: prediction, detection and monitoring. Nature Reviews Cancer 2008, 8, 268.

8. Rusling, J. F.; Kumar, C. V.; Gutkind, J. S.; Patel, V., Measurement of biomarker proteins for point-of-care early detection and monitoring of cancer. Analyst 2010, 135 (10), 2496-2511.

9. Kingsmore, S. F., Multiplexed protein measurement: technologies and applications of protein and antibody arrays. Nature Reviews Drug Discovery 2006, 5, 310.

10. Liu, J.; Tang, H.; Zhang, B.; Deng, X.; Zhao, F.; Zuo, P.; Ye, B.-C.; Li, Y., Electrochemical sensor based on molecularly imprinted polymer for sensitive and selective determination of metronidazole via two different approaches. Analytical and Bioanalytical Chemistry 2016, 408 (16), 4287-4295.

11. Arya, S. K.; Pui, T. S.; Wong, C. C.; Kumar, S.; Rahman, A. R. A., Effects of the Electrode Size and Modification Protocol on a Label-Free Electrochemical Biosensor. Langmuir 2013, 29 (22), 6770-6777.

12. Wang, T.; Fang, Y.; He, Z. Z., Electrochemical Quantitative Detection of Glial Fibrillary Acidic Protein Based on Molecularly Imprinted Polymer Sensor. Int J Electrochem Sc 2017, 12 (8), 7341-7350.

13. Huang, W.; Besar, K.; LeCover, R.; Dulloor, P.; Sinha, J.; Martínez Hardigree, J. F.; Pick, C.; Swavola, J.; Everett, A. D.; Frechette, J.; Bevan, M.; Katz, H. E., Label-free brain injury biomarker detection based on highly sensitive large area organic thin film transistor with hybrid coupling layer. Chemical Science 2014, 5 (1), 416-426.

14. Abeyrathne, C. D.; Huynh, D. H.; Lee, T. T.; Nguyen, T. C.; Nasr, B.; Chana, G.; Skafidas, E., GFAP Antibody Detection Using Interdigital Coplanar Waveguide Immunosensor. IEEE Sensors Journal 2016, 16 (9), 2898-2905. 
15. Song, J.; Dailey, J.; Li, H.; Jang, H.-J.; Zhang, P.; Wang, J. T.-H.; Everett, A. D.; Katz, H. E., Extended Solution Gate OFET-Based Biosensor for Label-Free Glial Fibrillary Acidic Protein Detection with Polyethylene Glycol-Containing Bioreceptor Layer. Adv Funct Mater 2017, 27 (20), 1606506.

16. Okumoto, S.; Jones, A.; Frommer, W. B., Quantitative Imaging with Fluorescent Biosensors. Annual Review of Plant Biology 2012, 63 (1), 663-706.

17. Morris, M. C.; Blondel, M., Editorial: Fluorescent biosensors. Biotechnology Journal 2014, 9 (2), 171-173.

18. Tamura, T.; Hamachi, I., Recent Progress in Design of Protein-Based Fluorescent Biosensors and Their Cellular Applications. ACS Chemical Biology 2014, 9 (12), 2708-2717.

19. Bauch, M.; Toma, K.; Toma, M.; Zhang, Q.; Dostalek, J., Plasmon-Enhanced Fluorescence Biosensors: a Review. Plasmonics 2014, 9 (4), 781-799.

20. Ibraheem, A.; Campbell, R. E., Designs and applications of fluorescent protein-based biosensors. Current Opinion in Chemical Biology 2010, 14 (1), 30-36.

21. Chernov, K. G.; Redchuk, T. A.; Omelina, E. S.; Verkhusha, V. V., Near-Infrared Fluorescent Proteins, Biosensors, and Optogenetic Tools Engineered from Phytochromes. Chem Rev 2017, 117 (9), 6423-6446.

22. Tung, C.-H.; Gerszten, R. E.; Jaffer, F. A.; Weissleder, R., A Novel Near-Infrared Fluorescence Sensor for Detection of Thrombin Activation in Blood. Chembiochem 2002, 3 (23), 207-211.

23. Cui, X. X.; Fan, Q.; Shi, S. J.; Wen, W. H.; Chen, D. F.; Guo, H. T.; Xu, Y. T.; Gao, F.; Nie, R. Z.; Harold, D. F.; Gordon, H. T.; Hou, C. Q.; Peng, B., A novel near-infrared nanomaterial with high quantum efficiency and its applications in real time in vivo imaging. Nanotechnology 2018, 29 (20), 205705.

24. Tsuboi, S.; Yamada, S.; Nakane, Y.; Sakata, T.; Yasuda, H.; Jin, T., Critical ReviewWater-Soluble Near-Infrared Fluorophores Emitting over $1000 \mathrm{~nm}$ and Their Application to In Vivo Imaging in the Second Optical Window (1000-1400 nm). ECS Journal of Solid State Science and Technology 2018, 7 (1), R3093-R3101.

25. Lakowicz, J. R., Principles of fluorescence spectroscopy. Springer; 3rd edition 1999.

26. Li, J. F.; Li, C. Y.; Aroca, R. F., Plasmon-enhanced fluorescence spectroscopy. Chem Soc Rev 2017, 46 (13), 3962-3979.

27. Fu, Y.; Zhang, J.; Lakowicz, J. R., Plasmon-Enhanced Fluorescence from Single Fluorophores End-Linked to Gold Nanorods. J Am Chem Soc 2010, 132 (16), 5540-+. 28. Lakowicz, J. R.; Ray, K.; Chowdhury, M.; Szmacinski, H.; Fu, Y.; Zhang, J.; Nowaczyk, K., Plasmon-controlled fluorescence: a new paradigm in fluorescence spectroscopy. Analyst 2008, 133 (10), 1308-1346.

29. Li, M.; Cushing, S. K.; Wu, N., Plasmon-enhanced optical sensors: a review. Analyst 2015, 140 (2), 386-406.

30. Emmanuel, F.; Samuel, G., Surface enhanced fluorescence. Journal of Physics D: Applied Physics 2008, 41 (1), 013001. 
31. Anger, P.; Bharadwaj, P.; Novotny, L., Enhancement and Quenching of Single-Molecule Fluorescence. Phys Rev Lett 2006, 96 (11), 113002.

32. Theodorou, I. G.; Jiang, Q.; Malms, L.; Xie, X.; Coombes, R. C.; Aboagye, E. O.; Porter, A. E.; Ryan, M. P.; Xie, F., Fluorescence enhancement from single gold nanostars: towards ultrabright emission in the first and second near-infrared biological windows. Nanoscale 2018, 10 (33), 15854-15864.

33. Theodorou, I. G.; Jawad, Z. A. R.; Jiang, Q.; Aboagye, E. O.; Porter, A. E.; Ryan, M. P.; Xie, F., Gold Nanostar Substrates for Metal-Enhanced Fluorescence through the First and Second Near-Infrared Windows. Chemistry of Materials 2017, 29 (16), 6916-6926.

34. Shan, F.; Su, D.; Li, W.; Hu, W.; Zhang, T., Hot spots based gold nanostar@SiO2@CdSe/ZnS quantum dots complex with strong fluorescence enhancement. AIP Advances 2018, 8 (2), 025219.

35. Maawy, A. A.; Hiroshima, Y.; Kaushal, S.; Luiken, G. A.; Hoffman, R. M.; Bouvet, M., Comparison of a chimeric anti-carcinoembryonic antigen antibody conjugated with visible or near-infrared fluorescent dyes for imaging pancreatic cancer in orthotopic nude mouse models. $J$. Biomed. Opt. 2013, 18 (12), 126016.

36. Haynes, C. L.; Van Duyne, R. P., Nanosphere Lithography: A Versatile Nanofabrication Tool for Studies of Size-Dependent Nanoparticle Optics. The Journal of Physical Chemistry B 2001, 105 (24), 5599-5611.

37. Tabatabaei, M.; Sangar, A.; Kazemi-Zanjani, N.; Torchio, P.; Merlen, A.; LagugnéLabarthet, F., Optical Properties of Silver and Gold Tetrahedral Nanopyramid Arrays Prepared by Nanosphere Lithography. The Journal of Physical Chemistry C 2013, 117 (28), 14778-14786.

38. Zheng, P.; Kasani, S.; Shi, X.; Boryczka, A. E.; Yang, F.; Tang, H.; Li, M.; Zheng, W.; Elswick, D. E.; Wu, N., Detection of nitrite with a surface-enhanced Raman scattering sensor based on silver nanopyramid array. Analytica Chimica Acta 2018.

39. Ruach-Nir, I.; Bendikov, T. A.; Doron-Mor, I.; Barkay, Z.; Vaskevich, A.; Rubinstein, I., Silica-Stabilized Gold Island Films for Transmission Localized Surface Plasmon Sensing. J Am Chem Soc 2007, 129 (1), 84-92.

40. Johnson, P. B.; Christy, R. W., Optical Constants of the Noble Metals. Physical Review B 1972, 6 (12), 4370-4379.

41. Foerch, C.; Niessner, M.; Back, T.; Bauerle, M.; De Marchis, G. M.; Ferbert, A.; Grehl, H.; Hamann, G. F.; Jacobs, A.; Kastrup, A.; Klimpe, S.; Palm, F.; Thomalla, G.; Worthmann, H.; Sitzer, M., Diagnostic Accuracy of Plasma Glial Fibrillary Acidic Protein for Differentiating Intracerebral Hemorrhage and Cerebral Ischemia in Patients with Symptoms of Acute Stroke. Clinical Chemistry 2012, 58 (1), 237.

42. Mayer, C. A.; Brunkhorst, R.; Niessner, M.; Pfeilschifter, W.; Steinmetz, H.; Foerch, C., Blood Levels of Glial Fibrillary Acidic Protein (GFAP) in Patients with Neurological Diseases. PLOS ONE 2013, 8 (4), e62101.

43. Luger, S.; Witsch, J.; Dietz, A.; Hamann, G. F.; Minnerup, J.; Schneider, H.; Sitzer, M.; Wartenberg, K. E.; Niessner, M.; Foerch, C., Glial Fibrillary Acidic Protein Serum Levels 
Distinguish between Intracerebral Hemorrhage and Cerebral Ischemia in the Early Phase of Stroke. Clinical Chemistry 2017, 63 (1), 377.

44. Ren, C.; Kobeissy, F.; Alawieh, A.; Li, N.; Li, N.; Zibara, K.; Zoltewicz, S.; GuingabCagmat, J.; Larner, S. F.; Ding, Y.; Hayes, R. L.; Ji, X.; Mondello, S., Assessment of Serum UCH-L1 and GFAP in Acute Stroke Patients. Sci Rep-Uk 2016, 6, 24588.

45. Abel, B.; Kabir, T. S.; Odukoya, B.; Mohammed, M.; Aslan, K., Enhancement of the colorimetric response of enzymatic reactions by thermally evaporated plasmonic thin films: application to glial fibrillary acidic protein. Analytical Methods 2015, 7 (3), 1175-1185. 46. Ma, Y. S.; Xu, G. H.; Wei, F. D.; Cen, Y.; Song, Y. Y.; Ma, Y. J.; Xu, X. M.; Shi, M. L.; Sohail, M.; Hu, Q., Carbon dots based immunosorbent assay for the determination of GFAP in human serum. Nanotechnology 2018, 29 (14), 145501.

47. Khetani, S.; Kollath, V. O.; Kundra, V.; Nguyen, M. D.; Debert, C.; Sen, A.; Karan, K.; Sanati-Nezhad, A., Polyethylenimine Modified Graphene-Oxide Electrochemical Immunosensor for the Detection of Glial Fibrillary Acidic Protein in Central Nervous System Injury. ACS Sens. 2018, 3 (4), 844-851.

48. Cheng, Y. N.; Stakenborg, T.; Van Dorpe, P.; Lagae, L.; Wang, M.; Chen, H. Z.; Borghs, G., Fluorescence Near Gold Nanoparticles for DNA Sensing. Anal. Chem. 2011, 83 (4), 13071314. 


\section{Chapter 5: Inexpensive Plasmonic Copper Materials}

\subsection{Localized Surface Plasmon Resonance of Copper Nanocubes}

\subsubsection{Introduction}

Localized surface plasmon resonance (LSPR) is widely utilized in optoelectronics, biosensing, solar energy conversion, photochromics, invisibility cloak and other applications. ${ }^{1-6}$ Plasmonic materials developed so far are typically made of gold and silver. The relative high cost of gold and silver hinders the commercialization of plasmonic materials. Hence it is significant to search for low-cost and earth-abundant materials for plasmonic applications. Copper is much less expensive than gold $(\$ 0.01 / \mathrm{g} \mathrm{Cu}$ vs. $\$ 40 / \mathrm{g} \mathrm{Au}){ }^{7-8}$ Unfortunately, $\mathrm{Cu}$ nanostructures give an impression of weak and broad LSPR band because of its large imaginary part of the dielectric function. ${ }^{9-10}$ There are at least two factors contributing to the imaginary part of the dielectric function in metals: (i) intrinsic energy loss due to electron collisions, and (ii) interband transitions. ${ }^{11-12}$ The intrinsic energy loss can be modelled as a frequency-independent constant for a free-electron-like metal; and the influence of interband transitions is delocalized on the frequency scale, but only gaining strength above a material-dependent energy threshold. Unfortunately, the LSPR band of a $\mathrm{Cu}$ nanosphere predicted by Mie theory shows a large spectral overlap with the interband transitions owing to a low energy threshold at around $2.1 \mathrm{eV}$, leading to severe plasmon damping. ${ }^{13-15}$

Despite an overall large imaginary part of the dielectric function for $\mathrm{Cu}$ in the wavelength range from the ultraviolet (UV) light through the near-infrared (NIR) regions, it is noted that there is a low-loss window between $620 \mathrm{~nm}(2 \mathrm{eV})$ and $720 \mathrm{~nm}(1.72 \mathrm{eV})$ where the imaginary part significantly drops and becomes comparable to those of $\mathrm{Ag}$ and $\mathrm{Au}$. This makes it possible to realize strong plasmon in $\mathrm{Cu}$ nanostructures in such a narrow spectral window $(620 \sim 720 \mathrm{~nm}$, or $2 \sim 1.72 \mathrm{eV})$. Van Duyne realized an intense and narrow LSPR peak at $698 \mathrm{~nm}(1.78 \mathrm{eV})$ on $\mathrm{Cu}$ nanotriangle arrays, which is comparable to the extinction spectral line shape of the $\mathrm{Ag}$ and $\mathrm{Au}$ nanotriangle arrays with similar dimensions. ${ }^{16}$ Sugawa demonstrated an intense LSPR peak at 675 $\mathrm{nm}(1.84 \mathrm{eV})$ with the $\mathrm{Cu}$ half-shell array. ${ }^{17}$ Surprisingly, the experimental results show that $\mathrm{Cu}$ nanocubes displayed an intense LSPR peak with a narrow and asymmetric spectral line shape at around $585 \mathrm{~nm}(2.12 \mathrm{eV})$ where interband transitions have started contributing to the energy loss. ${ }^{18-}$ ${ }^{20}$ This contradicts the previous understanding that interband transitions strongly damp surface plasmon. ${ }^{14,21}$ It is also noted that $\mathrm{Cu}$ nanocubes exhibited a single LSPR peak whereas Ag 
nanocubes displayed multiple, which are correlated with different plasmon modes with surface polarization charges at the corner, edge and facet of the geometry. ${ }^{22-27}$ Also, both $\mathrm{Cu}$ and Ag nanospheres displayed a single LSPR peak due to the dipolar plasmon mode although the LSPR of $\mathrm{Cu}$ nanospheres is much weaker than that of $\mathrm{Ag}$ counterparts. ${ }^{19,28}$ These discrepancies suggest that the origin of the single intense LSPR band with a narrow and asymmetric spectral line shape observed in $\mathrm{Cu}$ nanocubes may be much different from that of $\mathrm{Ag}$ nanocubes, which demands to be fully understood theoretically.

This study aims to shed light on the origin of the strong and narrow LSPR band of $\mathrm{Cu}$ nanocubes. To account for the influence of interband transitions, the dielectric function is decomposed into a free-electron component and an interband transitions component. The plasmon modes are calculated with the discrete dipole approximation (DDA) method for a free-electronlike $\mathrm{Cu}$ nanocube and a $\mathrm{Cu}$ nanocube under the influence of interband transitions, which suggests that all but one plasmon mode gets damped by the interband transitions. To unravel how interband transitions could interplay with the plasmon modes, a theoretical model is developed. According to this model, a free-electron-like nanocube displays multiple LSPR peaks corresponding to plasmon modes at the corner, edge, and facet. Under the influence of interband transitions, those plasmon modes get damped due to their spectral overlap with interband transitions, inducing a tail of broad background absorption. Only the plasmon mode near or below the threshold of interband transitions survives and maintains a free-electron-like spectral line shape. As a result, the cubic corner mode of a $\mathrm{Cu}$ nanocube with the lowest resonance energy near the threshold of interband transitions survives, and exhibits a distinct, strong and narrow LSPR peak. In addition, the extinction coefficient and the local electromagnetic field enhancement (EM) are investigated. We believe that the theoretical model developed in this study can be used to study plasmon modes for a nanoparticle of arbitrary shape under the influence of interband transitions, which will guide the design of plasmonic nanostructures, especially for inexpensive materials like $\mathrm{Cu}$.

\subsubsection{Methods}

\section{Chemicals and Materials}

Oleylamine (70\%), trioctylphosphine oxide (TOPO, 90\%), CuBr, toluene, n-hexane, acetone and ethanol were purchased from Sigma-Aldrich. All these chemicals were used as received without any further purification.

\section{Synthesis of $\mathrm{Cu}$ Nanocubes}

$\mathrm{Cu}$ nanocubes were synthesized with disproportionation of $\mathrm{CuBr} .{ }^{19} \mathrm{CuBr}(0.6 \mathrm{mmol})$ and TOPO ( $5 \mathrm{mmol})$ were dissolved into oleylamine $(2 \mathrm{~mL})$ in a three-neck flask with strong magnetic stirring at $80{ }^{\circ} \mathrm{C}$ under the nitrogen atmosphere. After $15 \mathrm{~min}$, the resulting solution was rapidly heated up to $210^{\circ} \mathrm{C}$ and refluxed at this temperature for $1 \mathrm{~h}$ before naturally cooling down to room temperature. The $\mathrm{Cu}$ nanocubes synthesized were separated and washed with hexane for two cycles 
of centrifugation and redispersion. Finally, $\mathrm{Cu}$ nanocubes were dispersed into toluene by ultrasonication for $30 \mathrm{~min}$.

\section{Characterizations}

Synthesized $\mathrm{Cu}$ nanocubes were characterized under a JEOL JSM-7600F scanning electron microscope (SEM) and a JEM 2100F transmission electron microscope (TEM) at an acceleration voltage of $200 \mathrm{kV}$. The extinction spectrum of $\mathrm{Cu}$ nanocubes was measured with a Shimadzu 2550 UV-Visible spectrometer (UV 2401/2, Shimadzu).

\section{DDA Calculations}

Optical extinction spectra, EM field distributions and enhancement factors were calculated using an open source code, DDSCAT 7.3, developed by Draine et al. ${ }^{29}$ In DDA, the target object is represented by an array of point dipoles. The electromagnetic scattering problem can be solved exactly for an incident wave interacting with these point dipoles. In our study, a total of 125,000 dipoles were used to represent a cubic shape with an edge length of $30 \mathrm{~nm}$. The EM field was extracted at each peak wavelength. All the data of dielectric functions used are from Johnson \& Christy. ${ }^{29}$

\section{Electron-Driven DDA (e-DDA) Calculations}

Electron energy loss spectroscopy (EELS) spectra were calculated using an open source code e-DDA v1.2, ${ }^{30}$ which was compiled based on the DDSCAT code developed by Draine et al. ${ }^{29}$ In our study using e-DDA, swift electron beams with an energy of $300 \mathrm{keV}$ were used to replace the incident light. To excite the corner plasmon mode, we directed the trajectory of incident electrons to pass by the corner of the cube; to excite the facet plasmon mode which is difficult to be excited optically, we directed the trajectory of incident electrons to pass by the facet of the cube.

\section{Finite-Difference Time-Domain (FDTD) Simulation}

Surface polarization charge densities were calculated by Lumerical FDTD Solutions 8.15. A total-field scattered-field (TFSF) was used as the input light source. A mesh size of $0.5 \mathrm{~nm}$ was used. The dielectric function of $\mathrm{Cu}$ was from Johnson and Christy. ${ }^{29}$ For a free-electron-like $\mathrm{Cu}$, the dielectric function was fitted using a Drude Model as shown in Eq. 1 with fitting parameters listed in Table S1. The background refractive index was fixed at 1.33. After the plasmon modes of free-electron-like $\mathrm{Cu}$ nanocube were determined by calculating the extinction spectrum, surface polarization charge densities were calculated at the peak wavelength of each mode for both freeelectron-like $\mathrm{Cu}$ nanocube and a $\mathrm{Cu}$ nanocube under the influence of interband transitions. 
(a)

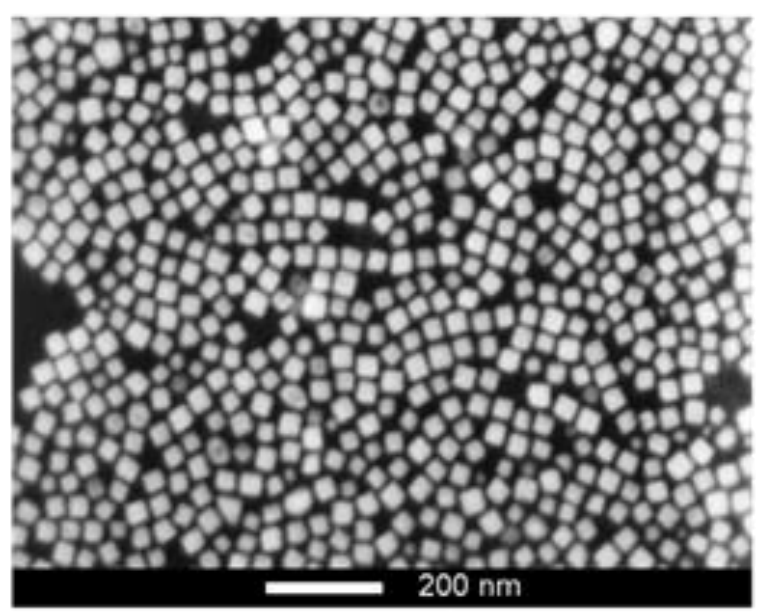

(b)

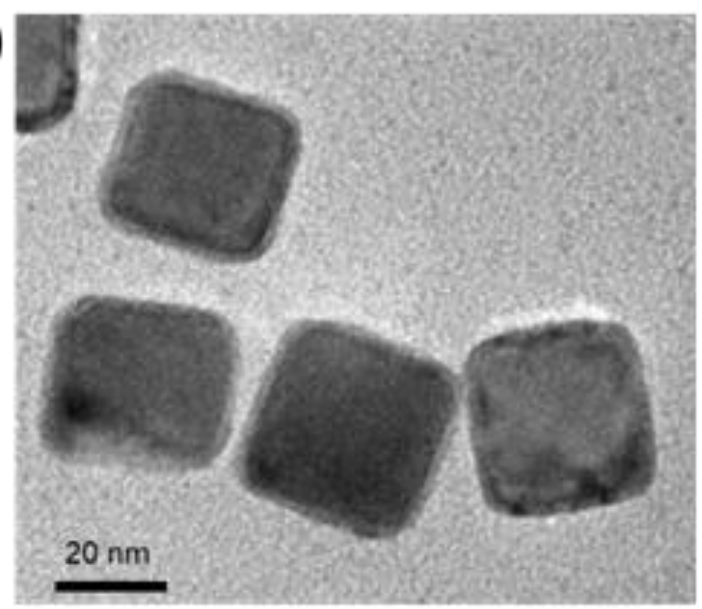

Figure 5.1 (a) SEM and (b) TEM images of synthesized Cu nanocubes with a medium size of $30 \mathrm{~nm}$ (see the size histogram in Figure 5.2).

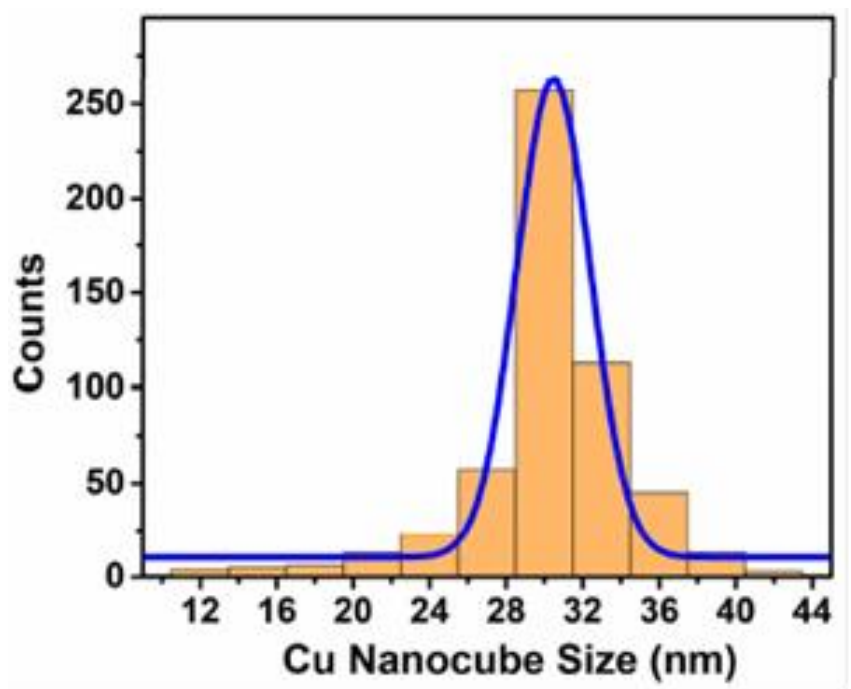

Figure 5.2 Histogram of $\mathrm{Cu}$ nanocubes showing the size distribution, which was statistically counted based on the SEM image in Fig. 1 (a) and fitted using a Gaussian function. The mean size is found to be $30 \mathrm{~nm}$ with a standard deviation $\sigma=1.92$.

\subsubsection{Results and Discussions}

\section{Optical Properties of Cu Nanocubes}

Figure 5.1 shows the synthesized $\mathrm{Cu}$ nanocubes. A medium edge size of $30 \mathrm{~nm}$ was obtained from Figure 5.2. The $\mathrm{Cu}$ nanocubes, which were suspended in toluene, displayed a single, intense, narrow and asymmetric LSPR peak, as shown with the solid blue curve in Figure 5.3. The interband transitions of $\mathrm{Cu}$ (shaded light blue region in Figure 5.3) exhibited an energy threshold close to the 
peak wavelength of $\mathrm{Cu}$ nanocubes. Surprisingly, the LSPR peak survived the damping by the interband transitions, and even exhibited a distinct asymmetric spectral line shape. The extinction spectrum of a single $\mathrm{Cu}$ nanocube with an edge length of $30 \mathrm{~nm}$ was also calculated using DDA and plotted with a dashed orange curve in Figure 5.3. It displayed a similar spectral line shape consistent with the measurement except for a slightly red-shifted LSPR peak position, which was ascribed to the roundness of the cubic corners. ${ }^{23}$ It is noted that the nanocubes used for all calculations in this paper were not rounded to ensure the consistency of data analysis with DDAand finite-difference time-domain (FDTD)-based numerical simulations and theoretical modelling. The calculation takes $30 \mathrm{~nm}$ of edge length for the nanocubes as well as the data of dielectric functions from Johnson \& Christy ${ }^{29}$ unless specified otherwise.

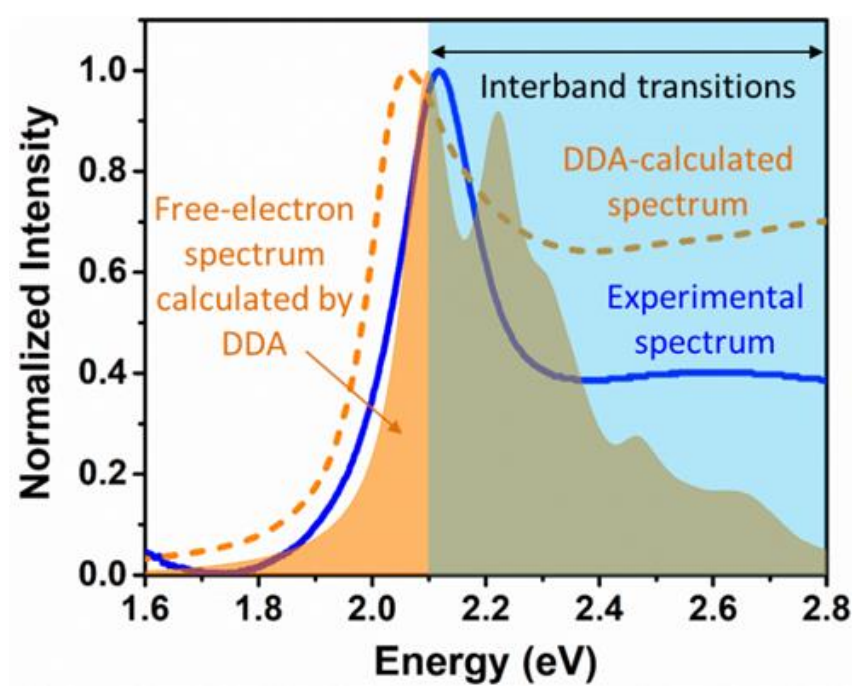

Figure 5.3 Extinction spectra of measured Cu nanocubes (blue solid curve) and a single Cu nanocube calculated by DDA (dashed orange curve). The orange shaded region showing the extinction spectrum of a free-electron-like (Drude Model) Cu nanocube calculated by DDA. The shaded light blue region for interband transitions of $\mathrm{Cu}$.

Table 5.1 Parameters of $\mathrm{Cu}, \mathrm{Au}$, and $\mathrm{Ag}$ used in a Drude Model in Equation (5.1) to model free-electron dielectric functions. These parameters are from literature. ${ }^{22,31-32}$

\begin{tabular}{|l|l|l|l|}
\hline & $\varepsilon_{\infty}$ & $\omega_{\mathrm{p}}(\mathrm{eV})$ & $\gamma(\mathrm{eV})$ \\
\hline $\mathrm{Cu}$ & 11.000 & 9.300 & 0.09550 \\
\hline $\mathrm{Au}$ & 9.700 & 8.890 & 0.07088 \\
\hline $\mathrm{Ag}$ & 4.039 & 9.172 & 0.02070 \\
\hline
\end{tabular}

\section{Free-Electron Plasmon Modes}

To examine what the free-electron-like extinction spectra look like without considering the interband transitions, the dielectric function is decomposed into a free-electron component and an interband transitions component. ${ }^{11}$ The free-electron component can be modelled using a Drude Model (DM). The DM formula is given by 


$$
\varepsilon(\omega)=\varepsilon_{\infty}-\frac{\omega_{p}^{2}}{\omega^{2}+i \gamma \omega}
$$

where $\varepsilon_{\infty}$ is the high frequency permittivity, $\omega_{p}$ is the plasma frequency, and $\gamma$ is the damping constant. The parameters used in the Drude Model for $\mathrm{Cu}, \mathrm{Au}$ and $\mathrm{Ag}$ were adopted from the literature $^{22,31-32}$ and tabulated in Table 5.1. Figure 5.4 shows the free-electron and interband transitions components of the dielectric functions for $\mathrm{Cu}, \mathrm{Au}$ and $\mathrm{Ag}$. In addition, the calculated free-electron-like extinction spectra for $\mathrm{Cu}, \mathrm{Au}$ and $\mathrm{Ag}$ nanocubes by DDA were plotted in the orange shaded region in Figures 5.3, 5.5(a) and 5.5(b), respectively. Interestingly, additional plasmon modes were observed in the case of a $\mathrm{Cu}$ nanocube (Figure 5.3) and an $\mathrm{Au}$ nanocube (Figure 5.5(a)), whereas an Ag nanocube was able to maintain its multiple plasmon modes observed initially regardless of interband transitions, which can be ascribed to the high interband transitions energy threshold of Ag. Comparison of DDA-calculated extinction spectra with and without the influence of interband transitions suggests that interband transitions indeed strongly interact with the plasmon modes, which reshapes the extinction spectrum of a $\mathrm{Cu}$ nanocube. In short, the LSPR peak of $\mathrm{Cu}$ nanocubes is affected by the interband transitions rather than the number of plasmonic peaks. A single narrow and asymmetric LSPR peak will occur if there is no damping by the interband transitions. 

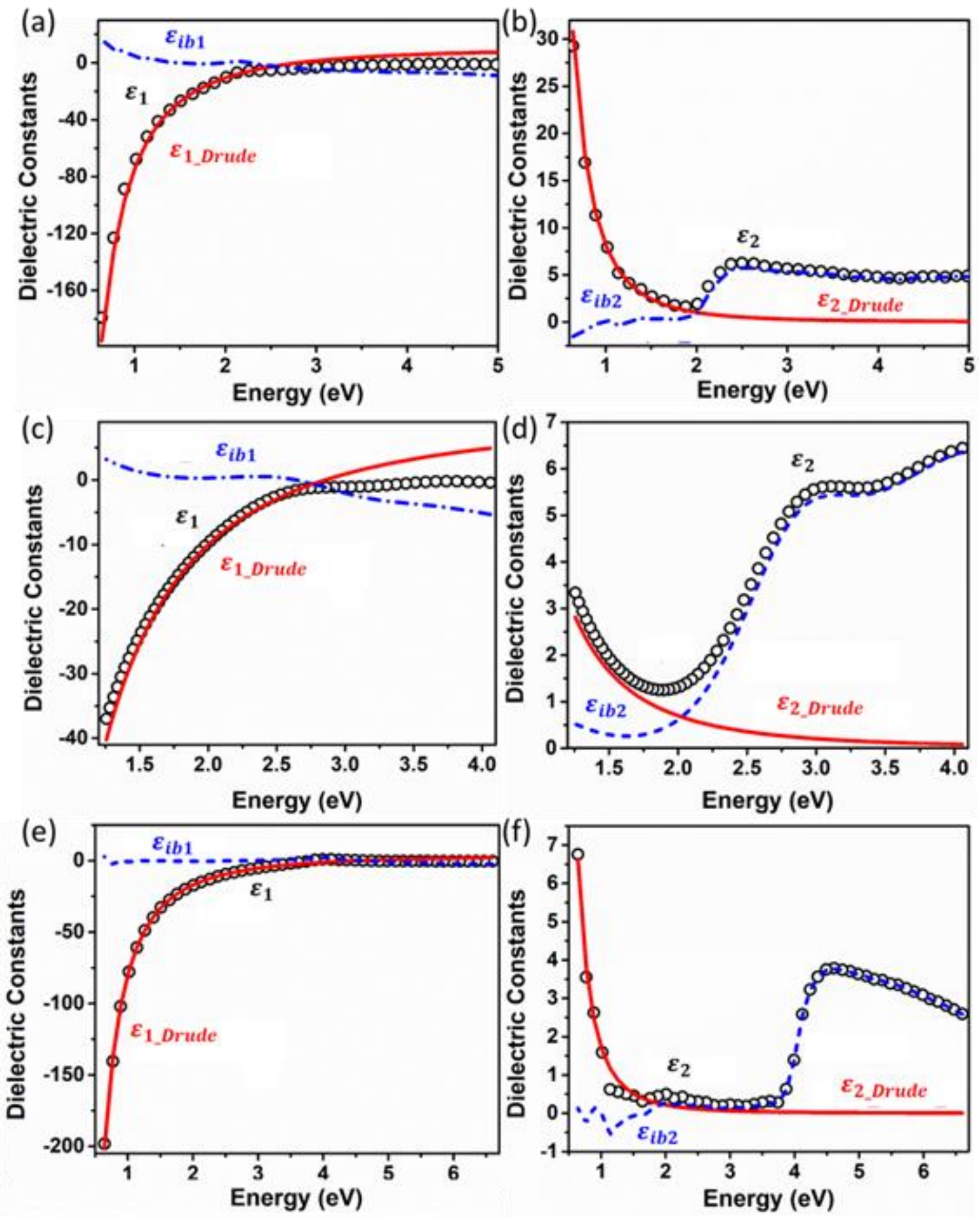

Figure 5.4 Decomposing experimentally measured dielectric function $\left(\varepsilon_{1}+i \varepsilon_{2}\right)$ from Johnson \& Christy into a free-electron component $\left(\varepsilon_{1 \_ \text {Drude }}+\mathrm{i} \varepsilon_{2_{-} \text {Drude }}\right)$ using the Drude Model in Equation (5.1) and an interband transitions component $\left(\varepsilon_{\mathrm{ib} 1}+\mathrm{i} \varepsilon_{\mathrm{ib} 2}\right)$. (a) and (b) for $\mathrm{Cu},(\mathrm{c})$ and (d) for $\mathrm{Au}$, (e) and (f) for $\mathrm{Ag}$. 

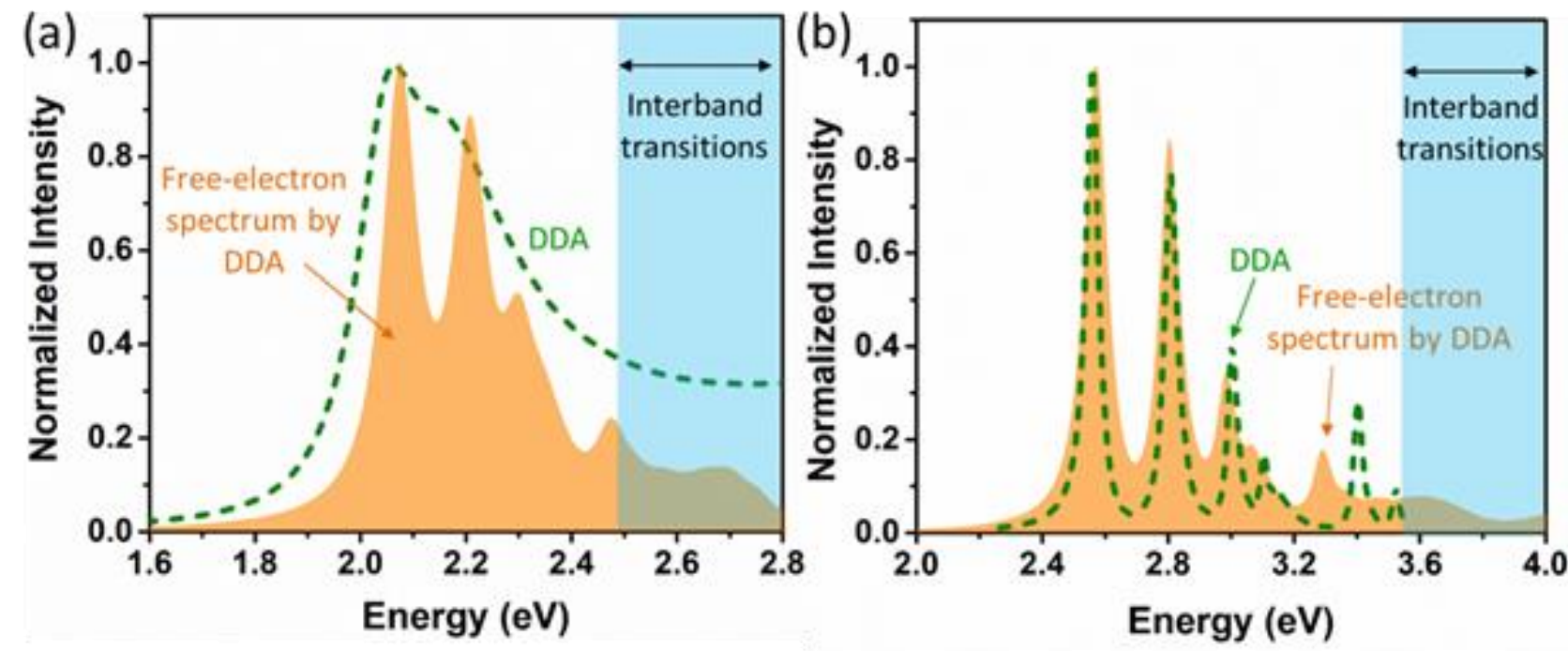

Figure 5.5 Calculated extinction spectra of (a) an Au nanocube and (b) an Ag nanocube with an edge length of $30 \mathrm{~nm}$. The olive dashed curves showing the DDA-calculated extinction spectra using the measured dielectric functions from Johnson \& Christy; the orange shaded regions showing the DDA-calculated extinction spectra of free-electron-like (Drude Model) nanocubes; the shaded light blue regions showing interband transitions.

\section{Examining the Possibility of Fano Resonance}

The spectral line shape of a $\mathrm{Cu}$ nanocube looks different from the symmetric Lorentzian line shape, which is predicted for small spherical nanoparticles by Mie theory when the damping constant is small. ${ }^{33}$ Instead, the distinct narrow and asymmetric spectral line shape reminds us to check whether the distinct asymmetric line shape of $\mathrm{Cu}$ nanocubes is attributed to Fano resonance.

While Fano resonance is ubiquitous in physics, it usually occurs in a two-level system in which coherent interaction between a discrete state and a continuum leads to a distinct asymmetric line shape. ${ }^{34}$ Although Fano resonance has been reported for the Au and Ag nanocubes, it was only possible in nanocube dimers where destructive interference occurs between highly confined and radiating gap plasmon modes or by a substrate-supported nanocube where the dark and bright plasmon modes are coupled with each other, ${ }^{22-23}$ which is not the case for $\mathrm{Cu}$ nanocubes in this work. So far very few studies have demonstrated Fano resonance in a single nanoplasmonic system except that interband transitions and nonlocal effects have been found to affect the spectral line shape of plasmon peaks. ${ }^{11,35-36}$ It is noteworthy that a single Pd nanodisk can support the intrinsic Fano resonance due to the interference between the surface plasmons and the interband transitions. ${ }^{36}$ Therefore, we conducted fitting for the measured and calculated extinction spectra using the Fano formula 


$$
\sigma_{\text {Fano }}(E)=\sigma_{0} \frac{(q+\varepsilon)^{2}}{1+\varepsilon^{2}}, \varepsilon=\frac{E-E_{0}}{\hbar\left(\frac{\gamma}{2}\right)}
$$

where $E=\hbar \omega$ is the photon energy, $q$ is the Fano parameter, $\sigma_{0}$ is the cross section far away from the Fano resonance. $\varepsilon$ represents the reduced energy with $E_{0}$ and $\gamma$ being the Fano resonance energy and line width, respectively. The Fano parameter $q$ describes the phase shift of the continuum, and is the key parameter characterizing the asymmetric spectral line shape. In fact, the measured and calculated extinction spectra of the $\mathrm{Cu}$ nanocubes cannot be fit using the Fano formula. This suggests that the narrow and asymmetric LSPR band of $\mathrm{Cu}$ nanocubes was not induced by Fano resonance.

\section{Normal Modes of a Cu Nanocube}

Fuchs has developed a general approach to calculate the normal modes for nanoparticles of arbitrary shape. ${ }^{37}$ When this approach is applied to plasmonic nanostructures, the normal modes refer to surface plasmon modes, which primarily contribute to optical absorption. For small metallic nanoparticles less than $60 \mathrm{~nm}$ (e.g, $30 \mathrm{~nm} \mathrm{Cu}$ nanocubes), absorption is predominately responsible for extinction. Hence this study does not distinguish between absorption and extinction while acknowledging the existence of a small contribution from scattering. Following Fuchs's method, ${ }^{37}$ a theoretical model is adapted herein to provide an insight into the interaction between surface plasmons and interband transitions, and predict the extinction spectrum of a $\mathrm{Cu}$ nanocube.

In the subwavelength regime, the retardation effect can be neglected safely. The susceptibility of a nanoparticle is then represented by the sum over all normal modes, which is given by

$$
\chi(\omega)=\frac{1}{4 \pi} \sum_{m} \frac{C(m)}{\left(\frac{\varepsilon}{\varepsilon_{h}}-1\right)^{-1}+n_{m}}, \sum_{m} C(m)=1
$$

where $m$ is the index of normal modes, $\varepsilon$ is the dielectric function of the nanoparticle under study, and $\varepsilon_{h}$ is the dielectric constant of the host environment. The normal mode resonance frequencies are determined by the depolarization factor $n_{m}$ with the absorption strength given by $C(m)$. For a nanosphere under uniform polarization, there is only a single mode thanks to the

symmetry of the geometry. The depolarization factor of a nanosphere is $n_{m}=\frac{1}{3}$, which results in the well-known resonance condition $\frac{\varepsilon}{\varepsilon_{h}}=-2$ for a dipole mode. ${ }^{13}$ For a nanocube, there are at least nine normal modes. However, only six modes have significant strength, accounting for all but $7 \%$ of the absorption. The depolarization factors and absorption strengths for the six predominate modes of a nanocube have been previously determined: ${ }^{37} n_{1}=0.193, n_{2}=0.251$, $n_{3}=0.294, n_{4}=0.396, n_{5}=0.605, n_{6}=0.719, C(1)=0.31, C(2)=0.31, C(3)=0.07$, $C(4)=0.07, C(5)=0.13, C(6)=0.04$, with the rest negligible. These modes have surface polarization charges distributed on different parts of the geometry. The first and second modes have the highest amplitudes at the corner, but they have the lowest resonance energy. Therefore, 
the corner modes can be most easily excited. The fifth and sixth modes have the highest resonance energies with surface polarization charges distributed on the facet, which makes them most difficult to be excited.

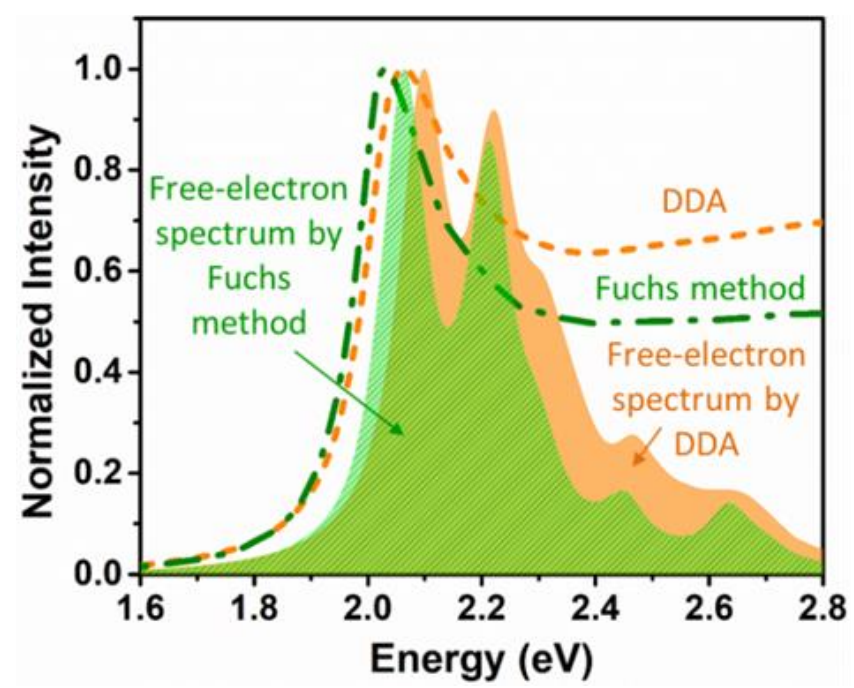

Figure 5.6 Calculated extinction spectra of a $\mathrm{Cu}$ nanocube by Fuchs method (olive color) and DDA method (orange color). The dashed and dash doted curves were calculated using the dielectric function from Johnson \& Christy; the shaded data was calculated using a free-electron dielectric function fitted by a Drude Model in Equation (5.1). The parameters used in Drude Model were tabulated in Table 5.1.

Based on the susceptibility of a nanoparticle above, the absorption coefficient in the electrostatic approximation can be calculated as follows

$$
\alpha(\omega)=4 \pi \frac{\omega}{c} \sqrt{\varepsilon_{h}} V \operatorname{Im}\langle\chi(\omega)\rangle
$$

where $c$ is the speed of light in vacuum and $V$ is the volume of a $\mathrm{Cu}$ nanocube. It is noted that the absorption coefficient is decided by the imaginary part of the susceptibility, which can be calculated by the sum over all six normal modes. Following Fuchs method, the absorption spectra of a $\mathrm{Cu}$ nanocube were calculated using both the measured dielectric function from Johnson \& Christy and the free-electron dielectric function fitted by a Drude Model in Equation (5.1). The absorption spectra calculated by the Fuchs method were consistent with the results by the DDA method (Figure 5.6). For the free-electron-like spectra calculated by both Fuchs and DDA methods, five out of the six dominant plasmon modes were uncovered with the remaining one beyond the energy range displayed. The plasmon modes, which were considerably overlapping with interband transitions, were significantly broadened and damped except for the one with a resonance frequency near the energy threshold of interband transitions. The calculation by Fuchs method further underscores the critical role of interband transitions that play in reshaping the spectral line shape.

Based on observations above, we were motivated to understand the mechanism of interactions between interband transitions and surface plasmons from a theoretical perspective. To begin with, 
we derived a general form for the absorption coefficient for a free-electron-like nanoparticle of arbitrary shape by combining Equations (5.1), (5.3), and (5.4), which is given by

$$
\begin{gathered}
\alpha(\omega)=\frac{\sqrt{\varepsilon_{h}} V}{c} \sum_{m} C(m) \frac{A_{0} \omega^{2} \Omega^{2} \gamma}{\left(\omega^{2}-\Omega^{2}\right)^{2}+(\gamma \omega)^{2}} \\
A_{0}=\frac{1}{n_{m}}+\frac{\varepsilon_{h}-1}{n_{m} \omega_{p}^{2}} \Omega^{2} \\
\Omega=\frac{\omega_{p}}{\sqrt{1+\left(\frac{1}{n_{m}}-1\right) \varepsilon_{h}}}
\end{gathered}
$$

If the damping constant $\gamma$, which is the frequency of electron collisions, is small, the absorption spectrum of a free-electron-like nanoparticle would have a symmetric Lorentzian spectral line shape. The resonance condition is established at $\omega_{\text {res }}=\Omega$, where the depolarization factor determines the resonance frequency. The line width can also be found as $\Gamma=\gamma$, which has an origin of free electron collisions.

When interband transitions are involved, a complex term $\varepsilon_{i b}(\omega)$ which was determined in Figure 5.4 is added to the Drude formula in Equation (5.1) to account for the contribution from interband transitions. The dielectric function is now represented by

$$
\begin{aligned}
& \varepsilon(\omega)=1-\frac{\omega_{p}^{2}}{\omega^{2}+i \omega \Gamma}+\varepsilon_{i b}(\omega) \\
& \varepsilon_{i b}(\omega)=\varepsilon_{i b 1}(\omega)+i \varepsilon_{i b 2}(\omega)
\end{aligned}
$$

where $\varepsilon_{i b 1}$ and $\varepsilon_{i b 2}$ represent the real and imaginary part of the component of interband transitions. The absorption coefficient for a nanoparticle under the influence of interband transitions can be similarly obtained by combining Equations (5.3), (5.4), (5.8), and (5.9), which is given by

$$
\alpha^{i b}(\omega)=\frac{\sqrt{\varepsilon_{h}} V}{c} \sum_{m} C(m) \frac{A_{1} \omega^{5}+A_{2} \omega^{4}+A_{3} \omega^{3}+A_{4} \omega^{2}}{\left[\left(\omega-\Delta \omega_{0}\right)^{2}-\Omega_{i b}{ }^{2}\right]^{2}+\left[\Gamma\left(\omega-\Delta \omega_{0}\right)\right]^{2}} \Omega_{i b}^{2}
$$

Parameters of $A_{s}(s=1,2,3,4), \Delta \omega_{0}, \Omega_{i b}$, and $\Gamma$ are given below

$$
\begin{gathered}
\Delta \omega_{0}=\frac{\gamma \varepsilon_{i b 2} \Omega_{i b}{ }^{2}}{2 \omega_{p}^{2}} \\
A_{1}=\frac{\left(n_{m}-1\right)\left(1+\varepsilon_{i b 1}\right)+n_{m} \varepsilon_{h}}{n_{m}\left(1+\varepsilon_{i b 1}\right)+\left(1-n_{m}\right) \varepsilon_{h}} \frac{\varepsilon_{i b 2}}{n_{m} \omega_{p}^{2}} \\
A_{2}=\frac{1-n_{m}}{n_{m}\left(1+\varepsilon_{i b 1}\right)+\left(1-n_{m}\right) \varepsilon_{h}} \frac{\gamma \varepsilon_{i b 2}^{2}}{n_{m} \omega_{p}^{2}} \\
A_{3} \approx-\frac{\varepsilon_{i b 2}}{n_{m}\left(1+\varepsilon_{i b 1}\right)+\left(1-n_{m}\right) \varepsilon_{h}} \\
A_{4}=\gamma \frac{2\left(1+\varepsilon_{i b 1}\right)-\frac{\varepsilon_{h}}{n_{m}}}{n_{m}\left(1+\varepsilon_{i b 1}\right)+\left(1-n_{m}\right) \varepsilon_{h}}
\end{gathered}
$$




$$
\begin{gathered}
\Omega_{i b}=\frac{\omega_{p}}{\sqrt{1+\left(\frac{1}{n_{m}}-1\right) \varepsilon_{h}+\varepsilon_{i b 1}}} \\
\Gamma=\frac{\varepsilon_{i b 2} \Omega_{i b}{ }^{2}}{n_{m} \omega_{p}^{2}}\left(\omega-\Delta \omega_{0}\right)+\gamma\left(1+\frac{\varepsilon_{i b 2}^{2} \Omega_{i b}{ }^{2}}{n_{m} \omega_{p}^{2}}\right)
\end{gathered}
$$

The expression for the absorption coefficient $\alpha^{i b}(\omega)$ in Equation (5.10) is very general. If only considering free electrons, then $\varepsilon_{i b 1}=\varepsilon_{i b 2}=0$. In this case, only $A_{4}$ survives with $\Delta \omega_{0}=0$, $\Omega_{i b}=\Omega$, and $\Gamma=\gamma$. This returns to the case when the absorption coefficient is obtained under a Drude Model. Therefore, all normal modes exhibit a symmetric Lorentzian line shape when the damping constant $\gamma$ is very small.

If interband transitions are taken into consideration, i.e. $\varepsilon_{i b 1}, \varepsilon_{i b 2} \neq 0$, then all $A_{s}$ terms survive, i.e. $A_{s} \neq 0$. But since $\varepsilon_{i b 1}$ and $\varepsilon_{i b 2}$ are very small at and below the threshold of interband transitions (Figure 5.4), the resonance frequency, which is approximately given as $\omega_{\text {res }}{ }^{i b}=\Omega_{i b}+$ $\Delta \omega_{0} \approx \omega_{\text {res }}$, would hardly undergo a shift. Neither would any significant broadening occur to the linewidth $\Gamma$ except for the radiative decay induced linewidth broadening. Therefore, interband transitions only mildly affect the normal modes at or below the energy threshold. These normal modes can well maintain their free-electron-like LSPR spectral features. Their resonance frequencies are basically determined by the shape-dependent depolarization factors. This is the reason why the LSPR observed on a $\mathrm{Cu}$ nanocube exhibits a free-electron-like extinction peak near the threshold of interband transitions.

Interband transitions and surface plasmons come into full play above the threshold of interband transitions where $\varepsilon_{i b 2}$ is significant, as shown in Figure 5.4(b), (d), and (f). Since $\varepsilon_{i b 1}$ is small over the entire energy range considered, $\Omega_{i b}$ can always be approximated as $\Omega$, which is then primarily dependent on the depolarization factor $n_{m}$. The most important factor contributing to the resonance frequency shift comes from $\varepsilon_{i b 2}$, which adds the term $\Delta \omega_{0}$ to the frequency shift. But if the intrinsic damping constant $\gamma$ is small, as is true for noble metals, the amount of frequency shift $\Delta \omega_{0}$ would still be small. Therefore, interband transitions only mildly shift the resonance frequency if any. However, the linewidth $\Gamma$ would be significantly broadened owing to an increase of $\varepsilon_{i b 2}$. The linewidth broadening would have two consequences: first, the resonance strength would decrease, which results in plasmon damping; second, the extinction spectrum would no longer exhibit a symmetric Lorentzian line shape. When the higher-order polynomials of $\omega$ adds to the numerator of the absorption coefficient, the spectral line shape would become more asymmetric with the tail titled upward toward the higher energy side. This is exactly the origin of the asymmetric extinction spectral line shape observed on $\mathrm{Cu}$ nanocubes. As the metal of $\mathrm{Cu}$ has a small intrinsic damping constant $\gamma=0.0950 \mathrm{eV},{ }^{31}$ the surviving plasmon mode near the threshold of interband transitions displays an intense and narrow LSPR peak. However, well above the energy threshold of interband transitions, surface plasmons get significantly damped, leaving a trail of broad background absorption, as shown in Figures 5.3 and 5.6. 
As the susceptibility $\chi(w)$ in Equation (5.3) has a general form, and is applicable to a nanoparticle with an arbitrary shape, it allows the absorption coefficient derived in Equation (5.10) to be generalized in the subwavelength regime by parameterizing all the terms:

$$
\alpha(\omega)=\sum_{m} C(m) \frac{A_{m 1} \omega^{5}+A_{m 2} \omega^{4}+A_{m 3} \omega^{3}+A_{m 4} \omega^{2}}{\left(\omega^{2}-B_{m 1}^{2}\right)^{2}+\left(B_{m 2} \omega\right)^{2}}
$$

where $m$ represents the number of resonances, $A_{m s}(s=1,2,3,4)$ are coefficients of higher-order polynomials of $w, B_{m 1}$, and $B_{m 2}$ determine the resonance frequency and spectral linewidth, respectively. To validate the generalized absorption coefficient, we applied it to fit the measured and DDA-calculated extinction spectra of $\mathrm{Cu}$ nanocubes. The generalized formula fits the extinction spectra perfectly (Figure 5.7) and allows us to extract the critical parameters, which are otherwise indirectly accessible in the extinction spectra such as the resonance frequency and linewidth as tabulated in Tables 2 to 4 . For the $\mathrm{Cu}$ nancubes synthesized, although only one major LSPR peak is observed at the resonance energy $w_{\text {res }}=2.12 \mathrm{eV}$ in Figure 5.7(a), they actually possess multiple plasmon modes which are damped but can be extracted from the fitting parameters in Table 2. A similar observation can also be made for DDA-calculated extinction spectrum in Figure 5.7(b) and Table 5.3. If the influence of interband transitions is removed, those otherwise damped plasmon modes can be recovered by a free-electron-like $\mathrm{Cu}$ nanocube, displaying the distinct extinction spectral features, as shown in Figure 5.7(c). The fitting parameters are tabulated in Table 4. In Figure 5.7(c), the two sharp and intense LSPR peaks with the lowest resonance energies are attributed to the most easily excitable corner plasmon modes, whereas a weak LSPR peak with the highest resonance energy is originated from the plasmon mode on the cubic facet, which is most difficult to be excited. 

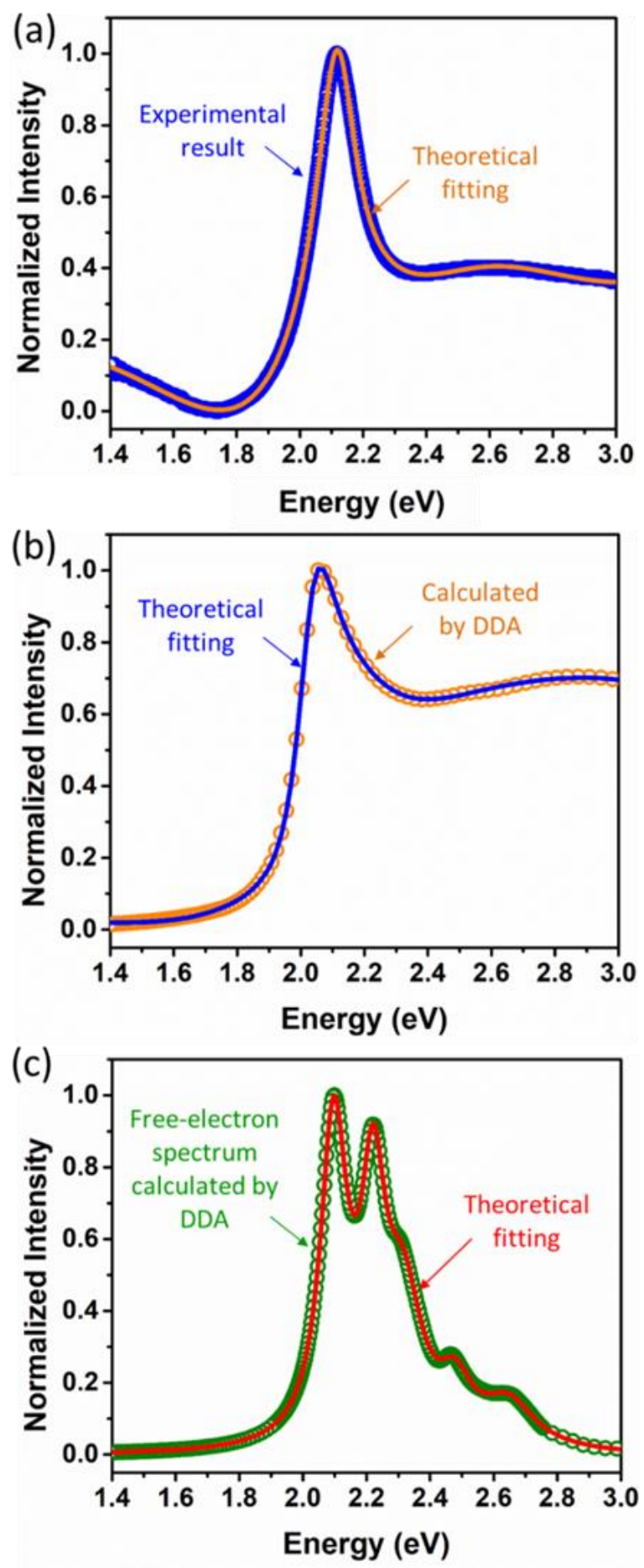

Figure 5.7 Extinction spectra fitting using the generalized absorption coefficient formula in Equation (5.18). (a) Measured extinction spectrum (thick blue) of synthesized Cu nanocubes and the fitted curve with the generalized absorption coefficient formula (orange); (b) Calculated extinction spectrum (orange hollow circles) of a Cu nanocube by DDA and the fitted curve (blue); (c) Calculated extinction spectrum (olive hollow circles) of a free-electron-like Cu nanocube by DDA and the fitted curve (red). The fitting parameters were tabulated in Table 5.2 to 5.4 . 
Table 5.2 Fitting parameters by the generalized absorption coefficient formula in Equation (5.18) for the measured extinction spectrum of synthesized Cu nanocubes.

\begin{tabular}{|l|l|l|l|l|l|l|}
\hline $\mathrm{m}$ & $\mathrm{A}_{\mathrm{m} 1}$ & $\mathrm{~A}_{\mathrm{m} 2}$ & $\mathrm{~A}_{\mathrm{m} 3}$ & $\mathrm{~A}_{\mathrm{m} 4}$ & $\mathrm{~B}_{\mathrm{m} 1}$ & $\mathrm{~B}_{\mathrm{m} 2}$ \\
\hline 1 & 3.59 & -14.71 & 13.87 & 2.65 & 2.12 & 0.15 \\
\hline 2 & -0.99 & 7.67 & -21.22 & 20.80 & 2.22 & 0.61 \\
\hline 3 & 17.87 & -23.91 & -13.23 & -16.19 & 2.30 & 1.34 \\
\hline 4 & -8.49 & -25.22 & -12.19 & 80.25 & 2.55 & 2.14 \\
\hline 5 & 1.41 & -5.76 & 33.76 & -40.28 & 2.81 & 3.21 \\
\hline 6 & -9.22 & 19.76 & -36.49 & -123.19 & 4.72 & 5.01 \\
\hline
\end{tabular}

Table 5.3 Fitting parameters by the generalized absorption coefficient formula in Equation (5.18) for the DDA-calculated extinction spectrum of a Cu nanocube

\begin{tabular}{|l|l|l|l|l|l|l|}
\hline $\mathrm{m}$ & $\mathrm{A}_{\mathrm{m} 1}$ & $\mathrm{~A}_{\mathrm{m} 2}$ & $\mathrm{~A}_{\mathrm{m} 3}$ & $\mathrm{~A}_{\mathrm{m} 4}$ & $\mathrm{~B}_{\mathrm{m} 1}$ & $\mathrm{~B}_{\mathrm{m} 2}$ \\
\hline 1 & 1.45 & -6.95 & 10.74 & -5.24 & 2.03 & 0.15 \\
\hline 2 & -2.35 & 13.06 & -24.27 & 15.17 & 2.20 & 0.54 \\
\hline 3 & 12.52 & -11.84 & -4.58 & -15.58 & 2.39 & 1.68 \\
\hline 4 & -5.74 & -19.19 & -9.16 & 78.74 & 2.60 & 2.00 \\
\hline 5 & 2.98 & -4.10 & 33.90 & -41.75 & 2.82 & 2.59 \\
\hline 6 & -6.53 & 21.49 & -35.89 & -123.54 & 4.53 & 1.79 \\
\hline
\end{tabular}

Table 5.4 Fitting parameters by the generalized absorption coefficient formula in Equation (5.18) for the DDA-calculated free-electron-like extinction spectrum of a Cu nanocube

\begin{tabular}{|l|l|l|l|l|l|l|}
\hline $\mathrm{m}$ & $\mathrm{A}_{\mathrm{m} 1}$ & $\mathrm{~A}_{\mathrm{m} 2}$ & $\mathrm{~A}_{\mathrm{m} 3}$ & $\mathrm{~A}_{\mathrm{m} 4}$ & $\mathrm{~B}_{\mathrm{m} 1}$ & $\mathrm{~B}_{\mathrm{m} 2}$ \\
\hline 1 & 0 & 0 & 0 & 0.04 & 2.10 & 0.10 \\
\hline 2 & 0 & 0 & 0 & 0.03 & 2.22 & 0.10 \\
\hline 3 & 0 & 0 & 0 & 0.12 & 2.32 & 0.15 \\
\hline 4 & 0 & 0 & 0 & 0.01 & 2.48 & 0.12 \\
\hline 5 & 0 & 0 & 0 & 0.07 & 2.65 & 0.20 \\
\hline 6 & 0 & 0 & 0 & -0.99 & 3.20 & 3.00 \\
\hline
\end{tabular}

\section{EELS Spectrum of a Cu Nanocube}

The single extinction peak with a narrow and asymmetric spectral line shape of a $\mathrm{Cu}$ nanocube is understood as a result of a shape-dependent depolarization factor and an interband transitionsinduced plasmon damping. Given that those damped plasmon modes only display weak spectral features in free-electron-like optical extinction spectra as shown in Figure 5.7, we are interested in probing them in order to give a full description of the plasmon modes of a $\mathrm{Cu}$ nanocube. Since those damped modes with higher resonance frequencies originate from plasmon modes on the facet, which are weakly coupled to light, they cannot be effectively optically excited. Instead of an optical excitation, these modes can be excited electronically. Therefore, we applied the electrondriven discrete dipole approximation method (e-DDA) to calculate the electron energy loss spectroscopy (EELS) spectra associated with those plasmon modes. In the e-DDA method, we can specify the trajectory of incident electrons and selectively excite different plasmon modes. For instance, when the incident electrons take the trajectory along the corner of a $\mathrm{Cu}$ nanocube, the 
corner modes can be excited. When parallel to the plane of a facet, the edge and facet modes can be excited. In our calculations, by guiding incident electrons to take two different trajectories, we were able to obtain two complementary spectra, as shown in Figure 5.8. The plasmon modes marked as (1) and (2) in Figure 5.8 can be excited either optically or electronically, whereas the plasmon modes marked as (3) and (4) can only be effectively excited electronically, since they are weakly coupled to light.

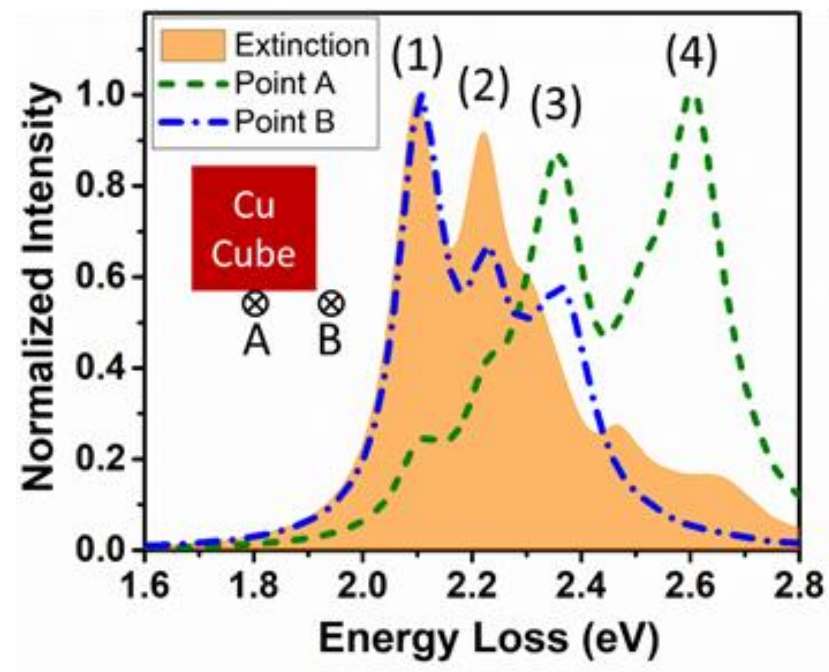

Figure 5.8 Comparison between EELS spectra and free-electron-like extinction spectrum of a $\mathrm{Cu}$ nanocube. The free-electron-like extinction spectrum (orange shaded region) displays two major peaks marked as (1) and (2). In EELS spectra: when the trajectory of incident electrons follows point A, plasmon modes with higher resonance energies marked as (3) and (4) can be excited, as shown by the olive dashed curve; similarly, plasmon modes with lower resonance frequencies marked as (1) and (2) can be excited as shown by the blue dash dotted curve when incident electrons follow the trajectory specified by point $B$.

\section{Surface Polarization Charge Density}

To visualize how interband transitions affect each plasmon mode identified in Figure 5.8, we calculated the surface polarization charge densities at each mode's peak wavelength using FDTD for a free-electron-like $\mathrm{Cu}$ nanocube and a $\mathrm{Cu}$ nanocube under the influence of interband transitions, as shown in Figure 5.9. Modes (1) through (4) in Figure 5.9 corresponds to the plasmon modes determined in Figure 5.8. From the calculated surface polarization charge densities, the free-electron-like $\mathrm{Cu}$ nanocube displays four well-distinguished plasmon modes, i.e. Mode (1): corner mode; Mode (2): corner mode with edge mode component; Mode (3): edge mode with corner and facet mode component; Mode (4): facet mode with edge mode component. These four modes are also confirmed by the study of EM field enhancement factors on a free-electron-like $\mathrm{Cu}$ nanocube as shown in Figure 5.10. Under the influence of interband transitions, however, surface polarization charge densities get drastically changed. As surface polarization charges on the cubic edge and facet cannot effectively sustain in the presence of interband transitions, they redistribute 
at the cubic corner, which ends up damping the edge and facet modes. Because the corner mode is a thermodynamically favorable state, it accumulates a large number of surface polarization charges and generates the most intense EM field, as shown in Figure 5.10(a).

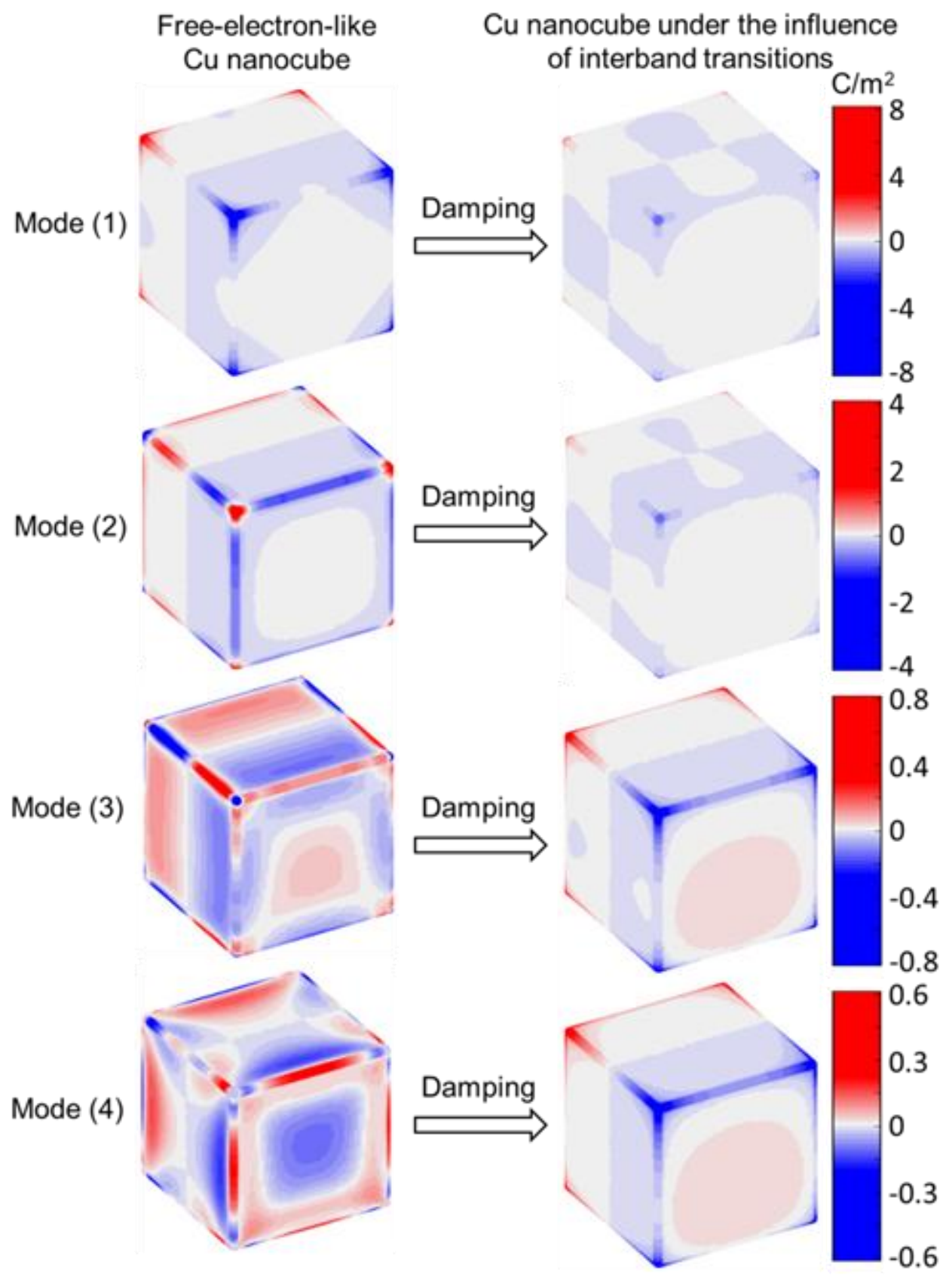

Figure 5.9 Surface polarization charge distribution calculated by FDTD where Mode (1) through (4) correspond to the modes determined in Figure 5.8. The left column shows the surface polarization charge distribution on a free-electron-like $\mathrm{Cu}$ nanocube; the right column shows that of a $\mathrm{Cu}$ nanocube under the influence of interband transitions. The scale bar indicates the surface polarization charge density in $\mathrm{C} / \mathrm{m}^{2}$. 

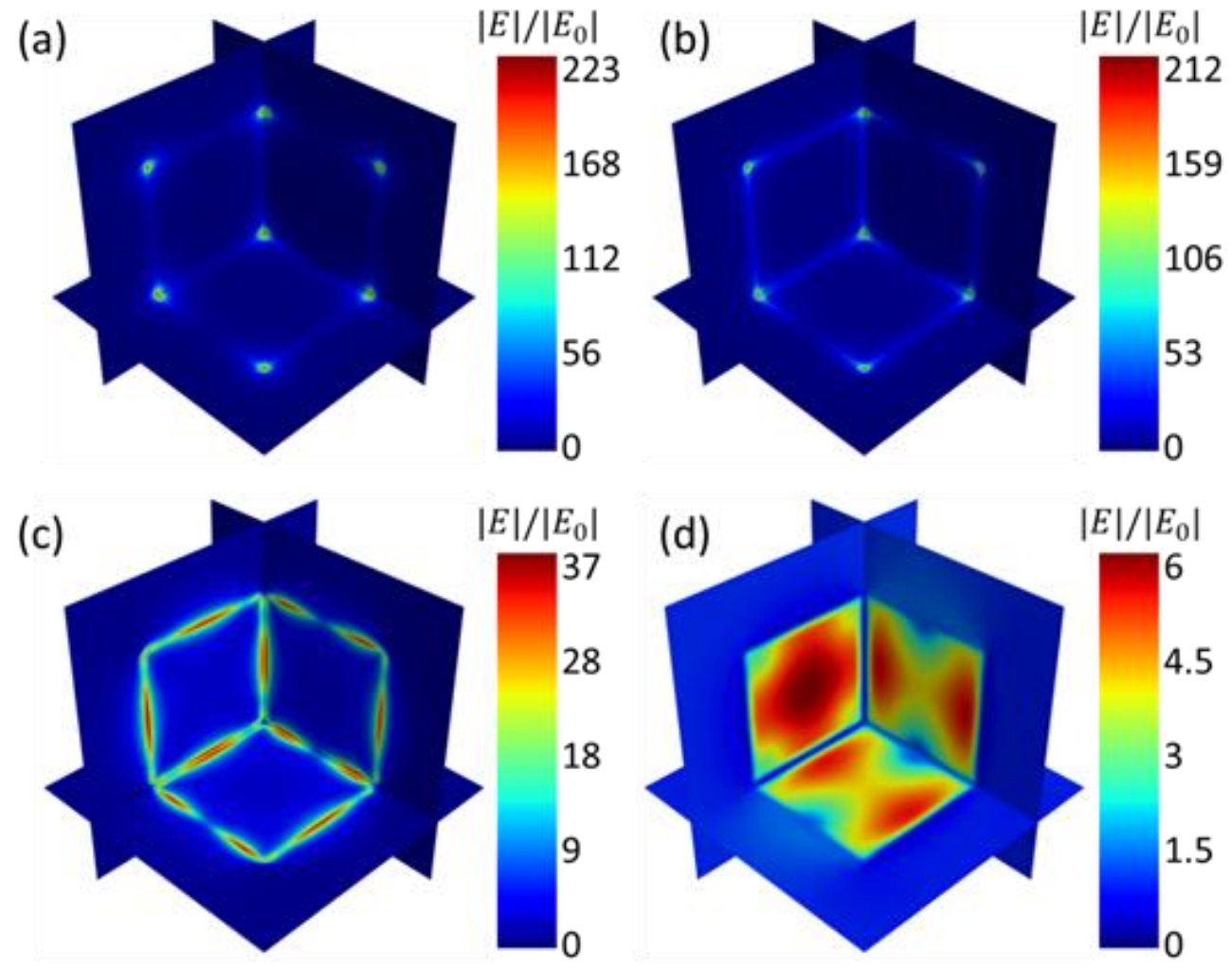

Figure 5.10 EM field distribution calculated by DDA for different plasmon modes supported on a freeelectron-like Cu nanocube. (a), (b), (c), and (d) corresponds to the plasmon mode marked as (1), (2), (3), and (4) in Figure 5.8, respectively. The EM field enhancement was calculated as $|E| /\left|E_{0}\right|$.

\section{Strong Plasmon of a Cu Nanocube}

From the measured and calculated extinction spectra of $\mathrm{Cu}$ nanocubes, the LSPR supported looks much stronger than what could be expected previously from $\mathrm{Cu}$ nanospheres. It is interesting to compare the LSPR of $\mathrm{Cu}$ nanocubes with those of Au nanocubes and nanospheres to check if a $\mathrm{Cu}$ nanostructure is an alternative plasmonic candidate to $\mathrm{Au}$ nanostructure. Therefore, the extinction spectrum of a $\mathrm{Cu}$ nanocube was calculated using DDA, and compared to those of the $\mathrm{Cu}$ nanosphere, $\mathrm{Au}$ nanocube and $\mathrm{Au}$ nansophere (Figure 5.11). The nanospheres and nanocubes calculated were modelled to be of the same volume, or equivalently the same effective radius. Therefore, for a cubic edge length of $30 \mathrm{~nm}$, the spherical diameter used in calculation is about 36 $\mathrm{nm}$. Apparently, the LSPR peak of a $\mathrm{Cu}$ nanocube is much stronger and sharper than that of a $\mathrm{Cu}$ nanosphere. While it is not as strong as a Au nanocube, it does have an extinction coefficient comparable to that of an Au nanosphere, making it a promising alternative for light management. 


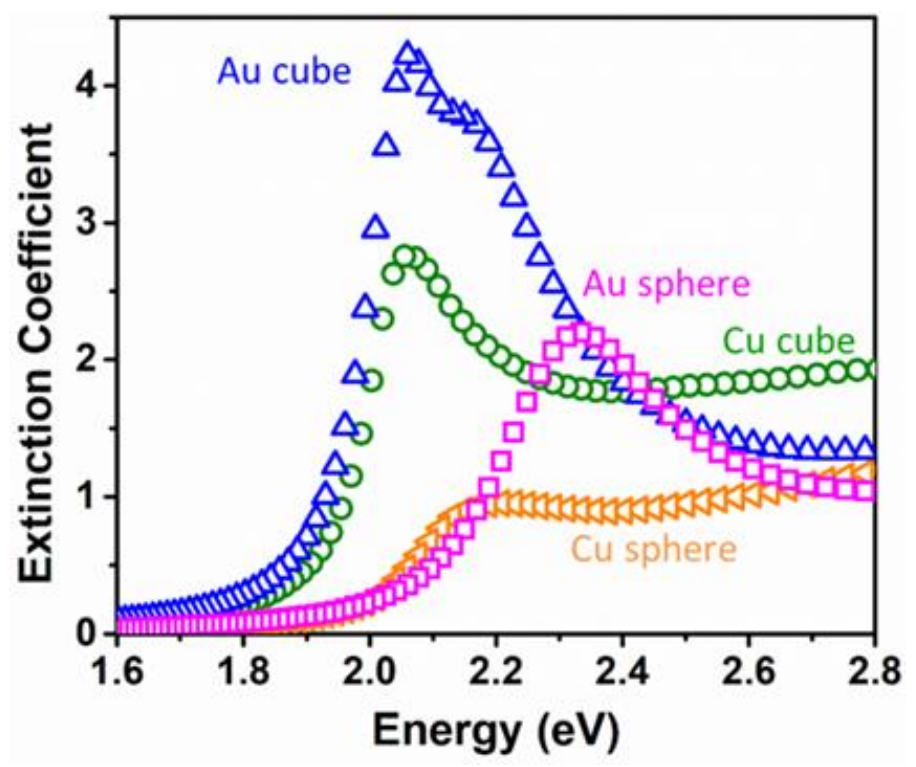

Figure 5.11 DDA-calculated extinction spectra of an Au nanocube, a Cu nanocube, an Au nanosphere, and a $\mathrm{Cu}$ nanosphere. The cubes and spheres calculated are of the same volume, i.e., an edge length of $30 \mathrm{~nm}$ for cubes and a diameter of $36 \mathrm{~nm}$ for spheres.

Finally, the EM field enhancement factors $\left(|\mathbf{E} / /| \mathbf{E}_{0} \mid\right)$ for the cubic corner mode of a $\mathrm{Cu}$ nanocube was calculated using DDA, and compared to the cubic corner mode of a Au nanocube, the dipole modes of a $\mathrm{Cu}$ nanosphere and an $\mathrm{Au}$ nanosphere, as shown in Figure 5.12. It is found that the EM field enhancement factor for the cubic corner LSPR mode of a $\mathrm{Cu}$ nanocube is less than half that of a Au nanocube, but it is 7.2 times as large as that of a dipole mode of a $\mathrm{Au}$ nanosphere. This indicates that $\mathrm{Cu}$ nanocubes are alternative plasmonic nanostructures owing to the low cost, comparable extinction coefficient, and large EM field enhancement factor as compared to the Au nanospheres. 

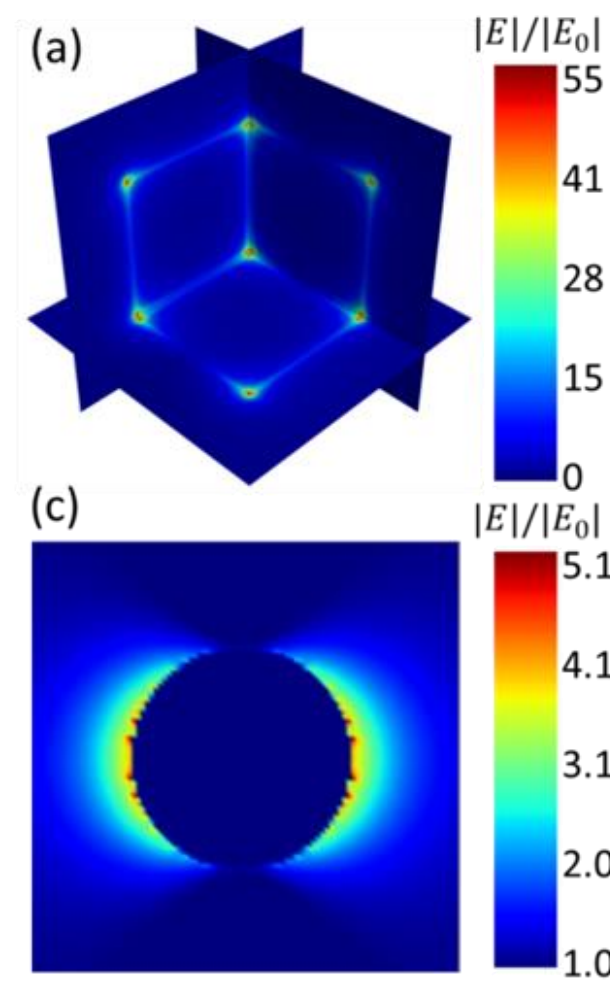
$|E| /\left|E_{0}\right|$
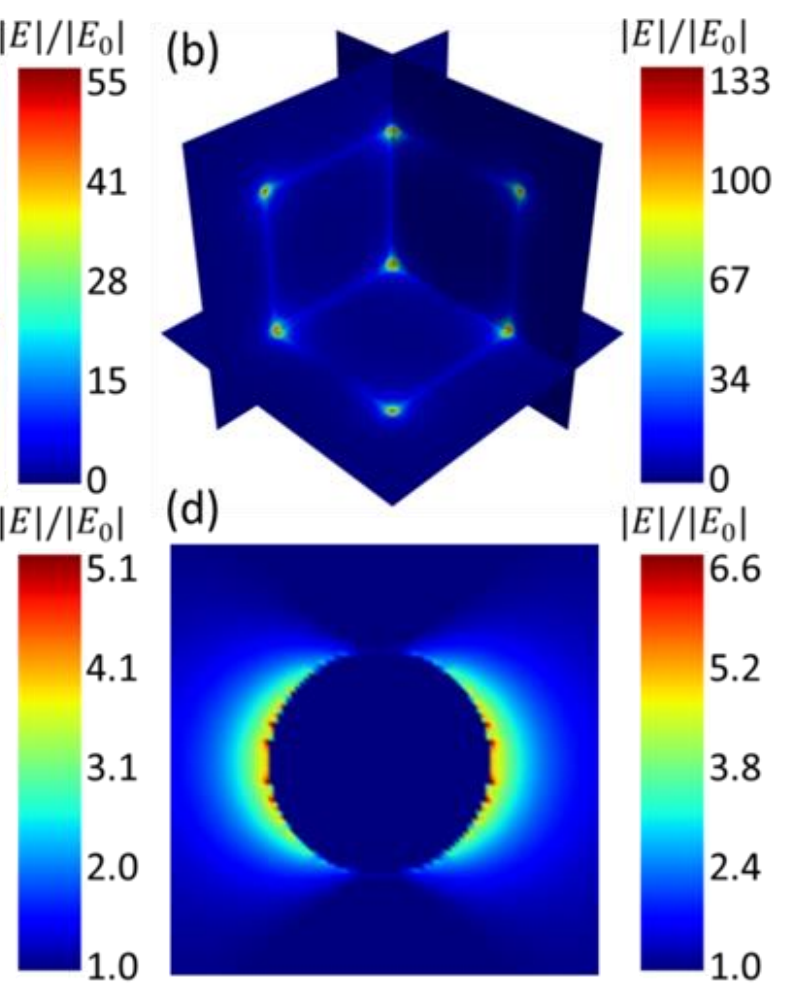

Figure 5.12 EM field distributions and enhancement factors $\left|E_{\mid} /\right| E_{0} \mid$ calculated by DDA for (a) a $\mathrm{Cu}$ nanocube, (b) an Au nanocube, (c) a Cu nanosphere, and (d) an Au nanosphere. The peak EM field intensity was used in the calculation of enhancement factor.

\subsubsection{Conclusion}

The LSPR of metallic nanocubes was investigated with experiments, numerical calculation and theoretical modeling. The theoretical model can be generalized to study the plasmon modes for a nanoparticle of arbitrary shape under the influence of interband transitions in the subwavelength regime, and guide future design of plasmonic nanostructures. In summary, $\mathrm{Cu}$ nanospheres generally show a weak and broad LSPR peak due to the severe damping by the interband transitions in the same spectral region of LSPR. In contrast, $\mathrm{Cu}$ nanocubes exhibit a single strong and narrow LSPR peak. Both numerical and theoretical calculations reveal that the LSPR peak is originated from the cubic corner mode, which is also the sole plasmon mode surviving interband transitions-induced damping. By decomposing the dielectric function into a free-electron component and an interband transitions component, a free-electron-like $\mathrm{Cu}$ nanocube has been found to support multiple plasmon modes with surface polarization charges distributed on the corner, edge, and facet of the geometry. Since the cubic corner plasmonic mode has the resonance energy near the threshold of interband transitions, it survives the damping from the interband transitions, maintaining a free-electron-like intense LSPR peak. However, other plasmon modes are spectrally overlapped with interband transitions, and they consequently get damped 
dramatically, leaving a broad background absorption titled upward at the higher energy side. In addition, the cubic corner mode of a $\mathrm{Cu}$ nanocube contributes to an extinction coefficient that is comparable to that of a $\mathrm{Au}$ nanosphere of the same volume, and yields a local EM field enhancement factor $\left(|\mathbf{E}| /\left|\mathbf{E}_{0}\right|\right)$ that is 7.2 times larger than that of the dipolar LSPR mode. In short, this work demonstrates that $\mathrm{Cu}$ nanocubes can be used as cost-effective plasmonic nanostructures. 


\section{Reference}

1. Du, W.; Wang, T.; Chu, H.-S.; Wu, L.; Liu, R.; Sun, S.; Phua, W. K.; Wang, L.; Tomczak, N.; Nijhuis, C. A., On-chip molecular electronic plasmon sources based on self-assembled monolayer tunnel junctions. Nature Photonics 2016, 10, 274.

2. $\quad$ Anker, J. N.; Hall, W. P.; Lyandres, O.; Shah, N. C.; Zhao, J.; Van Duyne, R. P., Biosensing with plasmonic nanosensors. Nature Materials 2008, 7, 442.

3. Andrew, T. L.; Tsai, H.-Y.; Menon, R., Confining Light to Deep Subwavelength Dimensions to Enable Optical Nanopatterning. Science 2009, 324 (5929), 917.

4. Li, J.; Cushing, S. K.; Meng, F.; Senty, T. R.; Bristow, A. D.; Wu, N., Plasmon-induced resonance energy transfer for solar energy conversion. Nature Photonics 2015, 9, 601.

5. Ni, X.; Wong, Z. J.; Mrejen, M.; Wang, Y.; Zhang, X., An ultrathin invisibility skin cloak for visible light. Science 2015, 349 (6254), 1310.

6. Gömöry, F.; Solovyov, M.; Šouc, J.; Navau, C.; Prat-Camps, J.; Sanchez, A., Experimental Realization of a Magnetic Cloak. Science 2012, 335 (6075), 1466.

7. Kawamura, G.; Alvarez, S.; Stewart, I. E.; Catenacci, M.; Chen, Z.; Ha, Y.-C., Production of Oxidation-Resistant Cu-Based Nanoparticles by Wire Explosion. Sci Rep-Uk 2015, 5, 18333.

8. Gawande, M. B.; Goswami, A.; Felpin, F.-X.; Asefa, T.; Huang, X.; Silva, R.; Zou, X.;

Zboril, R.; Varma, R. S., Cu and Cu-Based Nanoparticles: Synthesis and Applications in Catalysis. Chem Rev 2016, 116 (6), 3722-3811.

9. $\quad$ Liu, P.; Wang, H.; Li, X.; Rui, M.; Zeng, H., Localized surface plasmon resonance of Cu nanoparticles by laser ablation in liquid media. RSC Advances 2015, 5 (97), 79738-79745.

10. Gunalan, S.; Sivaraj, R.; Venckatesh, R., Aloe barbadensis Miller mediated green synthesis of mono-disperse copper oxide nanoparticles: Optical properties. Spectrochimica Acta Part A: Molecular and Biomolecular Spectroscopy 2012, 97, 1140-1144.

11. Anatoliy, P.; Gero von, P.; Uwe, K., Influence of interband electronic transitions on the optical absorption in metallic nanoparticles. Journal of Physics D: Applied Physics 2004, 37 (22), 3133.

12. Khurgin, J. B., Ultimate limit of field confinement by surface plasmon polaritons. Faraday Discussions 2015, 178 (0), 109-122.

13. Zayats, A. V.; Smolyaninov, I. I.; Maradudin, A. A., Nano-optics of surface plasmon polaritons. Physics Reports 2005, 408 (3), 131-314.

14. Wang, H.; Tam, F.; Grady, N. K.; Halas, N. J., Cu Nanoshells: Effects of Interband Transitions on the Nanoparticle Plasmon Resonance. The Journal of Physical Chemistry B 2005, 109 (39), 18218-18222.

15. Thi My Dung, D.; Thi Thu Tuyet, L.; Eric, F.-B.; Mau Chien, D., The influence of solvents and surfactants on the preparation of copper nanoparticles by a chemical reduction method. Advances in Natural Sciences: Nanoscience and Nanotechnology 2011, 2 (2), 025004. 
16. Chan, G. H.; Zhao, J.; Hicks, E. M.; Schatz, G. C.; Van Duyne, R. P., Plasmonic Properties of Copper Nanoparticles Fabricated by Nanosphere Lithography. Nano Letters 2007, 7 (7), 1947-1952.

17. Sugawa, K.; Tamura, T.; Tahara, H.; Yamaguchi, D.; Akiyama, T.; Otsuki, J.; Kusaka, Y.; Fukuda, N.; Ushijima, H., Metal-Enhanced Fluorescence Platforms Based on Plasmonic Ordered Copper Arrays: Wavelength Dependence of Quenching and Enhancement Effects. Acs Nano 2013, 7 (11), 9997-10010.

18. Yang, H.-J.; He, S.-Y.; Chen, H.-L.; Tuan, H.-Y., Monodisperse Copper Nanocubes: Synthesis, Self-Assembly, and Large-Area Dense-Packed Films. Chemistry of Materials 2014, $26(5), 1785-1793$.

19. Guo, H. Z.; Chen, Y. Z.; Cortie, M. B.; Liu, X.; Xie, Q. S.; Wang, X.; Peng, D. L., ShapeSelective Formation of Monodisperse Copper Nanospheres and Nanocubes via Disproportionation Reaction Route and Their Optical Properties. J. Phys. Chem. C 2014, 118 (18), 9801-9808.

20. Crane, C. C.; Wang, F.; Li, J.; Tao, J.; Zhu, Y. M.; Chen, J. Y., Synthesis of Copper-Silica Core-Shell Nanostructures with Sharp and Stable Localized Surface Plasmon Resonance. J. Phys. Chem. C 2017, 121 (10), 5684-5692.

21. Pirzadeh, Z.; Pakizeh, T.; Miljkovic, V.; Langhammer, C.; Dmitriev, A., PlasmonInterband Coupling in Nickel Nanoantennas. ACS Photonics 2014, 1 (3), 158-162.

22. Zhang, S.; Bao, K.; Halas, N. J.; Xu, H.; Nordlander, P., Substrate-Induced Fano Resonances of a Plasmonic Nanocube: A Route to Increased-Sensitivity Localized Surface Plasmon Resonance Sensors Revealed. Nano Letters 2011, 11 (4), 1657-1663.

23. Pellarin, M.; Ramade, J.; Rye, J. M.; Bonnet, C.; Broyer, M.; Lebeault, M.-A.; Lermé, J.; Marguet, S.; Navarro, J. R. G.; Cottancin, E., Fano Transparency in Rounded Nanocube Dimers Induced by Gap Plasmon Coupling. Acs Nano 2016, 10 (12), 11266-11279.

24. Ruppin, R., Plasmon frequencies of cube shaped metal clusters. Zeitschrift für Physik D Atoms, Molecules and Clusters 1996, 36 (1), 69-71.

25. Zhang, K. J.; Da, B.; Ding, Z. J., LSP modes of Ag nanocube and dimer studied by DDA simulation. Surface and Interface Analysis 2016, 48 (11), 1256-1262.

26. Cortie, M. B.; Liu, F.; Arnold, M. D.; Niidome, Y., Multimode Resonances in Silver Nanocuboids. Langmuir 2012, 28 (24), 9103-9112.

27. Mazzucco, S.; Geuquet, N.; Ye, J.; Stéphan, O.; Van Roy, W.; Van Dorpe, P.; Henrard, L.; Kociak, M., Ultralocal Modification of Surface Plasmons Properties in Silver Nanocubes. Nano Letters 2012, 12 (3), 1288-1294.

28. Mogensen, K. B.; Kneipp, K., Size-Dependent Shifts of Plasmon Resonance in Silver Nanoparticle Films Using Controlled Dissolution: Monitoring the Onset of Surface Screening Effects. The Journal of Physical Chemistry C 2014, 118 (48), 28075-28083.

29. Johnson, P. B.; Christy, R. W., Optical Constants of the Noble Metals. Physical Review B 1972, 6 (12), 4370-4379. 
30. Bigelow, N. W.; Vaschillo, A.; Iberi, V.; Camden, J. P.; Masiello, D. J., Characterization of the Electron- and Photon-Driven Plasmonic Excitations of Metal Nanorods. Acs Nano 2012, 6 (8), 7497-7504.

31. Zeman, E. J.; Schatz, G. C., An accurate electromagnetic theory study of surface enhancement factors for silver, gold, copper, lithium, sodium, aluminum, gallium, indium, zinc, and cadmium. The Journal of Physical Chemistry 1987, 91 (3), 634-643.

32. Ehrenreich, H.; Philipp, H. R., Optical Properties of Ag and Cu. Physical Review 1962, 128 (4), 1622-1629.

33. Fan, X.; Zheng, W.; Singh, D. J., Light scattering and surface plasmons on small spherical particles. Light: Science \&Amp; Applications 2014, 3, e179.

34. Fano, U., Effects of Configuration Interaction on Intensities and Phase Shifts. Physical Review 1961, 124 (6), 1866-1878.

35. García de Abajo, F. J., Nonlocal Effects in the Plasmons of Strongly Interacting Nanoparticles, Dimers, and Waveguides. The Journal of Physical Chemistry C 2008, 112 (46), 17983-17987.

36. Pakizeh, T.; Langhammer, C.; Zorić, I.; Apell, P.; Käll, M., Intrinsic Fano Interference of Localized Plasmons in Pd Nanoparticles. Nano Letters 2009, 9 (2), 882-886.

37. Fuchs, R., Theory of the optical properties of ionic crystal cubes. Physical Review $B$ 1975, $11(4), 1732-1740$. 


\subsection{Film-Coupled Copper Nanocube for Near-Infrared Fluorescence}

\section{Enhancement}

\subsubsection{Introduction}

Copper as a potential alternative low-cost plasmonic metal has found increasing research interest. ${ }^{1-5}$ However, the weak and broad localized surface plasmon (LSPR) resonance observed on most $\mathrm{Cu}$ nanoparticles significantly compromises the performance. ${ }^{4,6}$ Broadening of the LSPR peak usually occurs because of an intrinsic energy loss caused by electron collisions and radiative decay. But for $\mathrm{Cu}$ nanoparticles, the interband transition also contributes to plasmon broadening and damping owing to the spectral overlap between the interband transition and the LSPR band. ${ }^{7-}$

${ }^{8}$ Recently, by tailoring $\mathrm{Cu}$ nanoparticles into cubic shape, the cubic corner LSPR mode shows up with a sharp and narrow spectral line shape thanks to the spectral separation between the LSPR band and the interband transition. ${ }^{9-11}$ The observed LSPR mode on $\mathrm{Cu}$ nanocubes display quite a few superior properties over Au nanospheres, such as a larger extinction coefficient and a stronger local electromagnetic (EM) field. Nevertheless, the application of $\mathrm{Cu}$ nanocubes is severely restraint by the limited spectral tunability of the cubic corner LSPR mode, which is largely determined by the shape-dependent depolarization factor. ${ }^{12}$

The spectral tunability of a metallic nanoparticle can be greatly extended by plasmonic coupling, ${ }^{13-14}$ notably the film-coupled plasmonic nanocubes, ${ }^{15-18}$ which is an important genre of nanostructured metamaterials. Different from the coupling of plasmonic dimers through electric dipoles, the film-coupled plasmonic nanocube system is featured by strong magnetic dipoles due to the excitation of the plasmonic cavity mode at the gap region. Not only can the plasmonic cavity mode be effective excited by coupling the incident light with the magnetic dipole, the mode also displays strong EM field confined at the gap region. By manipulating the gap, the film-coupled nanocube system has found a lot of applications, such as engineering electromagnetic metasurfaces with controlled reflectance, ${ }^{18}$ enhancing the near field, ${ }^{17}$ controlling radiative decay processing, ${ }^{15}$ probing sub-picometer variation, ${ }^{19}$ and even accessing the quantum regime of plasmonics. ${ }^{20-22}$

By fabricating film-coupled $\mathrm{Cu}$ nanocube system, the merits of $\mathrm{Cu}$ nanocube such as a low cost and the excitation of the plasmonic cavity mode can be exploited to build near-infrared (NIR) fluorescent sensor devices. NIR fluorescent sensing is highly favored for its large signal-to-noise ratio (SNR) in the biological window. ${ }^{23}$ However, NIR quantum emitters often have a poor quantum efficiency less than $5 \%$. The film-coupled $\mathrm{Cu}$ nanocube system offers a potential solution, as the excited plasmonic cavity mode can give rise to a strong near field as well as an increased local optical density of states, which could potentially drastically enhance both the excitation rate and the quantum efficiency of a NIR quantum emitter. 
Therefore, in this research, a systematic study of the optical properties of the film-coupled $\mathrm{Cu}$ nancube system and its capability of enhancing NIR fluorescence emission is conducted based on numerical simulations using finite-difference time-domain (FDTD) method. Since $\mathrm{Cu}$ is subject to oxidation in air, an insulator layer, such as silica, is designed to coat on the $\mathrm{Cu}$ nanocube and the $\mathrm{Cu}$ film. It also serves as a spacer layer to control the separation distance between the $\mathrm{Cu}$ nanocube and the $\mathrm{Cu}$ film.

\subsubsection{Methods}

Finite-difference time-domain method is implemented throughout the paper to study the $\mathrm{Cu}$ film-coupled $\mathrm{Cu}$ nanocube system using commercially available software Lumerical FDTD Solutions. A mesh override region with a mesh size of $1 \mathrm{~nm}$ was imposed around the nanostructure. The dielectric function of $\mathrm{Cu}$ used was from Johnson \& Christy. ${ }^{24} \mathrm{~A}$ refractive index of 1.52 was given to the silica layer. The nanostructure system was excited using a plane wave, which allows back reflection spectra, radiation pattern, electromagnetic field data, and surface polarization charges to be calculated. The system was also excited by an electric dipole source placed inside the gap, which enables the calculation of quantum efficiency and radiative \& nonradiative decay rate. Boundary conditions of Perfectly Matched Layer (PML) were imposed on all six boundaries of the simulation region. 

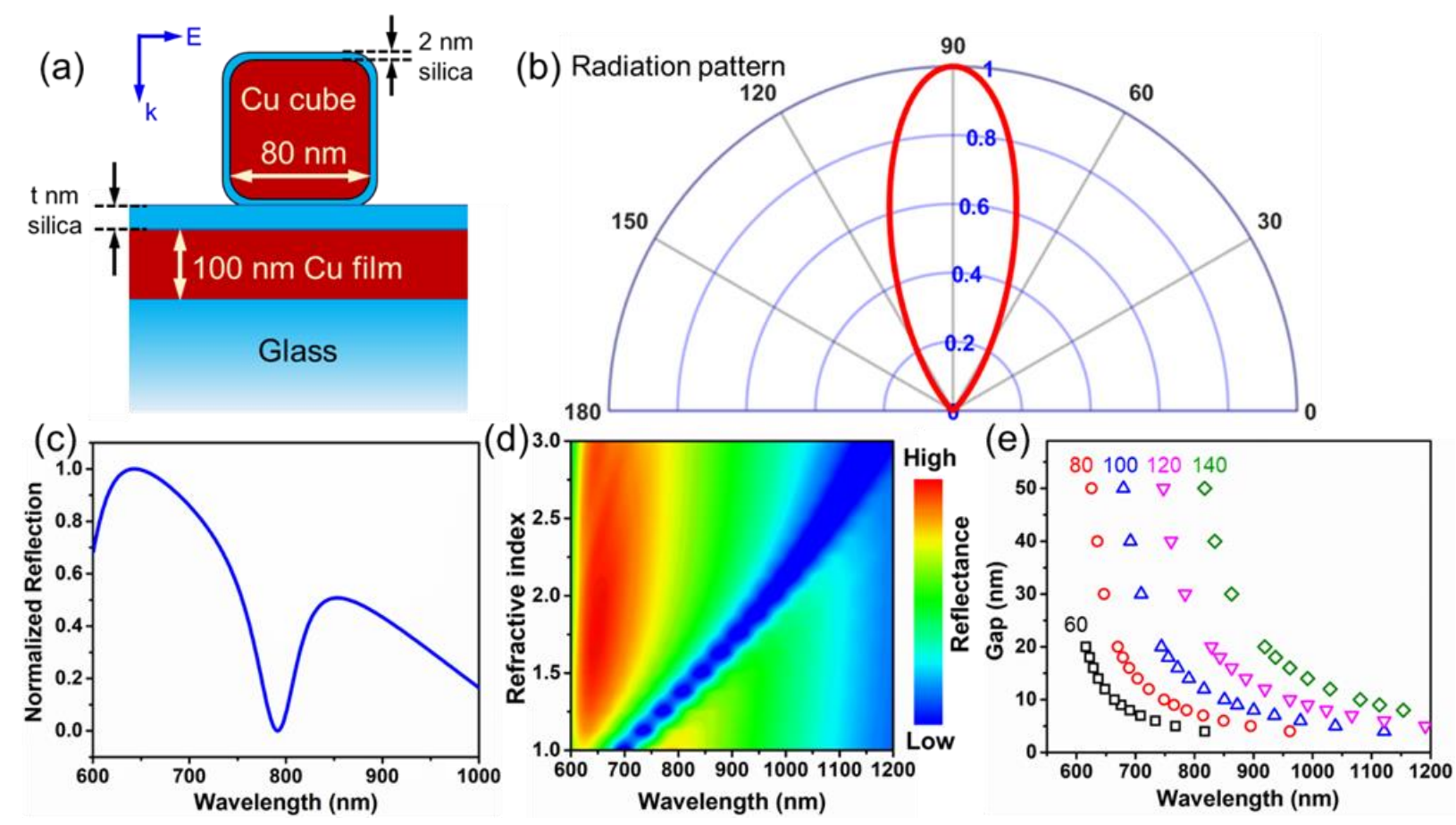

Figure 5.13 (a) Scheme of the film-coupled Cu nanocube system; (b) the radiation pattern, (c) the reflection spectrum, and (d) the separation materials refractive index-dependent reflection spectra for a $\mathrm{Cu}$ nanocube separated $8 \mathrm{~nm}$ away from the underlying $\mathrm{Cu}$ film; (e) the LSPR peak evolution for $\mathrm{Cu}$ nanocubes with varying silica gap and edge length of $\mathrm{Cu}$ nanocubes.

\subsubsection{Results and Discussion}

\section{Plasmonic Cavity Mode}

$\mathrm{A} \mathrm{Cu}$ nanocube coated with a silica layer of $\sim 2 \mathrm{~nm}$ was place on a $\mathrm{Cu}$ film but separated by a silica layer with varying thickness, as shown in Figure 5.12(a). The film-coupled $\mathrm{Cu}$ nanocube with a gap of $8 \mathrm{~nm}$ exhibited a reflection dip at around $780 \mathrm{~nm}$ and radiated largely in the surfacenormal direction at the resonance frequency, as shown in Figure 5.12(b) and (c). The reflection dip was identified as the plasmonic cavity mode, which could be spectrally tuned over a wide wavelength range from $620 \mathrm{~nm}$ to $1200 \mathrm{~nm}$ by either changing the refractive index of the separation material or varying the gap and edge length of the $\mathrm{Cu}$ nanocube, as shown in Figure 5.12(d) and (e).

The plasmonic cavity mode originates from Fabry-Pérot-like resonance. The gap region between the two facing surfaces of the $\mathrm{Cu}$ nanostructure supports transmission line type of resonances but modified by the plasmonic dispersion of $\mathrm{Cu}^{25} \mathrm{After}$ incidence, the EM waves can be guided into the transmission line (i.e. the gap) and self-adjusted to satisfy boundary conditions. Due to the large effective impedance mismatch at the edge of the gap region, most of the EM 
waves get reflected back into the gap and further adjust themselves to the transverse boundary conditions, with other leaky EM waves contributing to the far-field scattering. The interference of the waves before and after reflection at the gap edge results in Fabry-Pérot-like resonance, generating the plasmonic cavity mode.

An obvious advantage of the film-coupled nanocube system as compared to the conventional transmission line is that the $\mathrm{Cu}$ nanocube is not only involved in forming the transmission line type of resonance, but also acts as an antenna, which can effectively absorb the incidence EM radiation owing to its large absorption coefficient and transfers it to the plasmonic cavity mode. The surface polarization charge density shown in Figure 5.13(a) and (b) further underscores the merits of the film-coupled nanocube system, where the induced surface polarization charges on a free-standing $\mathrm{Cu}$ nanocube redistribute from cubic corners (Figure 5.13(a)) to the cubic bottom surface (Figure 5.13(b)) after the coupling between a $\mathrm{Cu}$ nanocube and the $\mathrm{Cu}$ film. The resulting plasmonic cavity mode not only displays an intense electric field at the edge of the gap region due to the induced surface polarization charges, as shown in Figure 5.13(c) and (d), but also generates a strong magnetic field at the center of the gap thanks to the fictitious magnetic current density induced along the periphery of the gap region. Therefore, the plasmonic cavity mode is basically a localization of the EM field whose electric and magnetic components oscillate spatially at the gap region. ${ }^{26}$

(a)

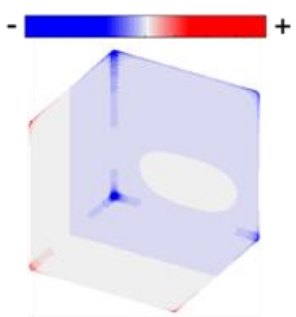

(b)

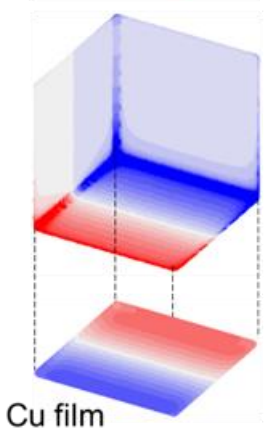

(c)

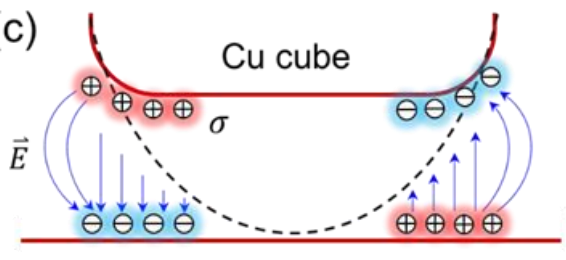

Cu film

(d)

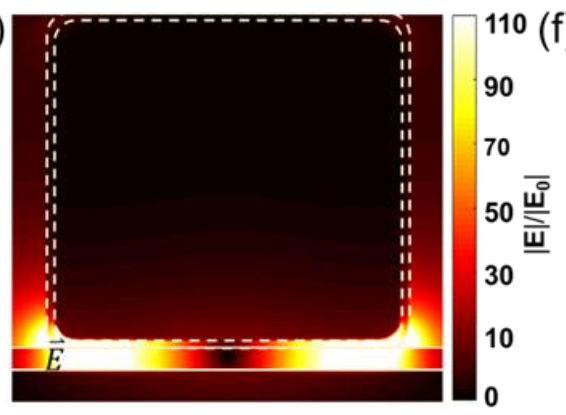

(e)
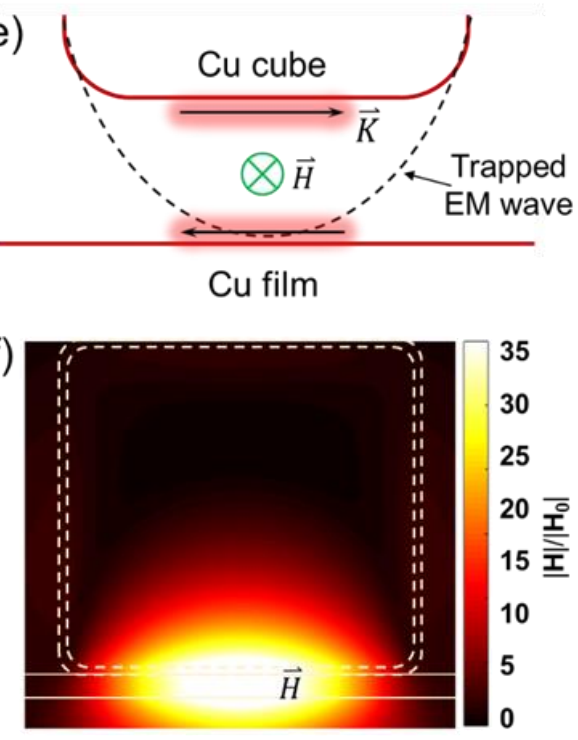

Figure 5.14 Surface polarization charge distribution for (a) a single Cu nanocube and (b) a film-coupled Cu nanocube; (c) scheme of induced surface polarization charge density (d) the corresponding electric field; (e) scheme of fictitious magnetic current density and (f) the corresponding magnetic field. $\mathrm{Cu}$ nanocube used is $\sim 80 \mathrm{~nm}$ in each dimension; the silica gap is $\sim 8 \mathrm{~nm}$; (a), (b), (d), and (f) were calculated at the plasmon resonance wavelength. 
The localization of the EM field and the spatial oscillation between the electric and magnetic field at the gap have two consequences. First, the film-coupled nanocube system is equivalent to a magnetic conductor, which can effectively couple with incident light through the magnetic dipole thanks to the excitation of fictitious magnetic current density. ${ }^{15,18}$ Second, the local optical density of states is significantly increased because of the plasmonic cavity mode, giving rise to a large Purcell factor to modify the spontaneous decay rate of quantum emitters, ${ }^{16}$ as will be shown in Figure 5.14(c). As the localized EM energy is being converted alternatively between electric and magnetic energy, which is a typical behavior of the Fabry-Pérot resonator, the energy loss only occurs either because of skin effect and finite conductivity of the metal, or through the leaky mode, which implies a large quality factor. ${ }^{26}$ Therefore, the local optical density of states at the gap region can be strongly modified by the Fabry-Pérot-like behavior of the film-coupled nanocube system.

\section{Fluorescence Enhancement of Near-Infrared Quantum Emitter}

Given the observed intense electric field and the Fabry-Pérot-like behavior at the gap region, the film-coupled $\mathrm{Cu}$ nanocube system was applied to enhance the fluorescence emission of a nearinfrared (NIR) quantum emitter, which can be modelled as a point electric dipole. Typically, the fluorescence emission enhancement $\left(\gamma_{e m} / \gamma_{e m}^{0}\right)$ consists of excitation rate enhancement $\left(\gamma_{\text {ext }} / \gamma_{\text {ext }}^{0}\right)$ and quantum efficiency enhancement $\left(\eta / \eta_{0}\right):{ }^{27}$

$$
\frac{\gamma_{e m}}{\gamma_{e m}^{0}}=\frac{\gamma_{e x t}}{\gamma_{e x t}^{0}} \frac{\eta}{\eta_{0}}
$$

The excitation enhancement depends on the local excitation electric field and the dipole orientation $(\vec{n})$ with respect to the electric field $(\vec{E})$ :

$$
\frac{\gamma_{\text {ext }}}{\gamma_{\text {ext }}^{0}}=\frac{|\vec{n} \cdot \vec{E}|^{2}}{\left|\vec{n} \cdot \overrightarrow{E_{0}}\right|}
$$

The maximum excitation rate enhancement can be achieved by allowing the dipole to orient in the direction of the electric field. And in this study, we consider the maximum achievable fluorescence emission enhancement by aligning the dipole in the electric field's direction.

Since a NIR quantum emitter usually has an intrinsic quantum efficiency $\eta_{0}$ less than $5 \%$, we modelled an electric dipole with an intrinsic quantum efficiency $\eta_{0}=0.01$ as a starting point in this study. The final quantum efficiency $\eta$ is defined as the radiative decay rate $\gamma_{\text {rad }}$ normalized by the total decay rate $\gamma$ which also includes the energy loss $\gamma_{a b s}$ due to absorption of the metal:

$$
\eta=\frac{\gamma_{\text {rad }}}{\gamma}=\frac{\gamma_{\text {rad }}}{\gamma_{\text {rad }}+\gamma_{a b s}+\gamma_{n r}}
$$

where the intrinsic nonradiative decay rate $\gamma_{n r}$ is assumed to be unaffected. 
Considering the intrinsic quantum efficiency $\eta_{0}$, which is given by

$$
\eta_{0}=\frac{\gamma_{r a d}^{0}}{\gamma_{r a d}^{0}+\gamma_{n r}^{0}}
$$

By combining Equation (5.21) and Equation (5.22), the quantum efficiency enhancement can be calculated as

$$
\frac{\eta}{\eta_{0}}=\frac{\frac{\gamma_{\text {rad }}}{\gamma_{\text {rad }}^{0}}}{\frac{\gamma_{\text {rad }}}{\gamma_{\text {rad }}^{0}} \eta_{0}+\frac{\gamma_{a b s}}{\gamma_{\text {rad }}^{0}}+\left(1-\eta_{0}\right)}
$$

where $\gamma_{\text {rad }} / \gamma_{\text {rad }}^{0}$ is the normalized radiative decay rate and can be obtained from the power monitor groups around the dipole in FDTD simulation software, $\gamma_{a b s} / \gamma_{r a d}^{0}$ is the normalized nonradiative decay rate due to Ohmic losses and can be obtained from the power monitor groups around the dipole-Au nanopyramid system.

FDTD simulations were then implemented to calculate the quantum efficiency enhancement following Equation (5.23) of a NIR quantum emitter, which was placed at the center plane parallel to the underlying $\mathrm{Cu}$ film but with varying lateral position at the gap region, as shown in Figure 5.14(a). Since the film-coupled nanocube system in Figure 5.14(a) displays a plasmonic cavity mode at $\sim 780 \mathrm{~nm}$ as shown in Figure 5.12(c), both the excitation and emission wavelength of the quantum emitter was set at $780 \mathrm{~nm}$ for simplicity.

Due to the intense electric field and the increased local optical density of states, the quantum emitter experienced large enhancement in its quantum efficiency and spontaneous decay rate. The two-dimensional (2D) distribution on the plane at the center of the gap (represented by a dashed red line in Figure 5.14(a)) of the quantum efficiency, Purcell factor, and fluorescence enhancement are shown in Figure 5.14(b), (c), and (d). Despite the overall large enhancement, spatial variations existed, which is probably caused by the spatial oscillation of the localized EM energy. ${ }^{26}$ The spatial profile of the fluorescence emission enhancement indicates that maximum fluorescence enhancement is achieved near the edges of the gap region, as shown in Figure 5.14(d). Identification of the optimal position for fluorescence enhancement will guide design of fluorescent biosensor devices. Therefore, in the following study of the gap-size effects, the quantum emitter is placed near the edge of the gap to harvest the largest possible enhancement, as shown in Figure 5.14(a). 
(a) Electric dipole emission

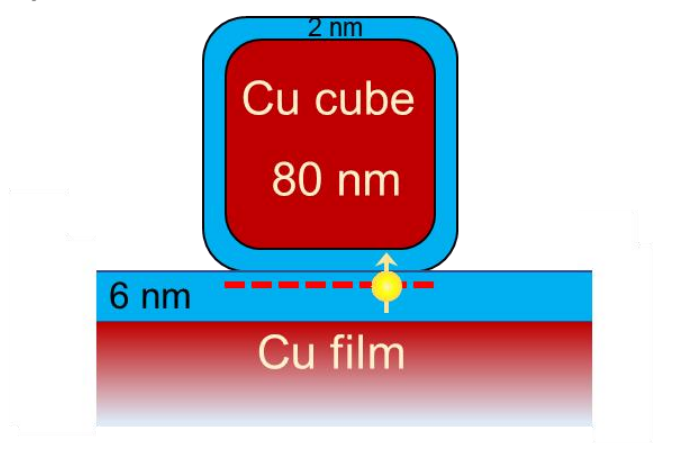

(b) Quantum efficiency $\eta / \eta_{0}\left(\eta_{0}=\mathbf{0 . 0 1}\right)$

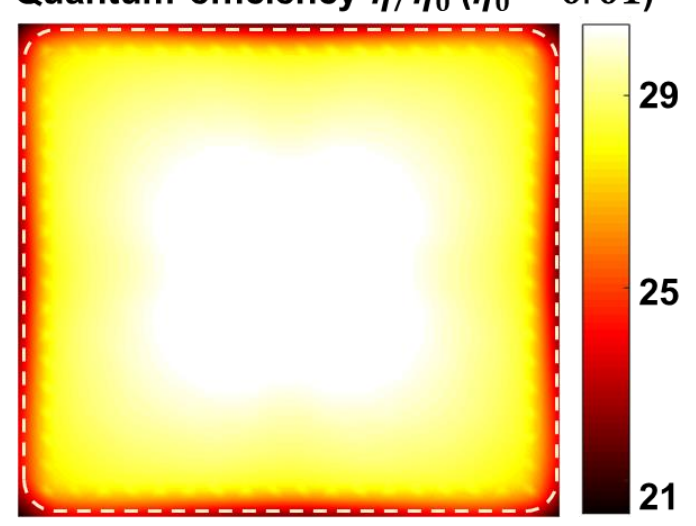

21

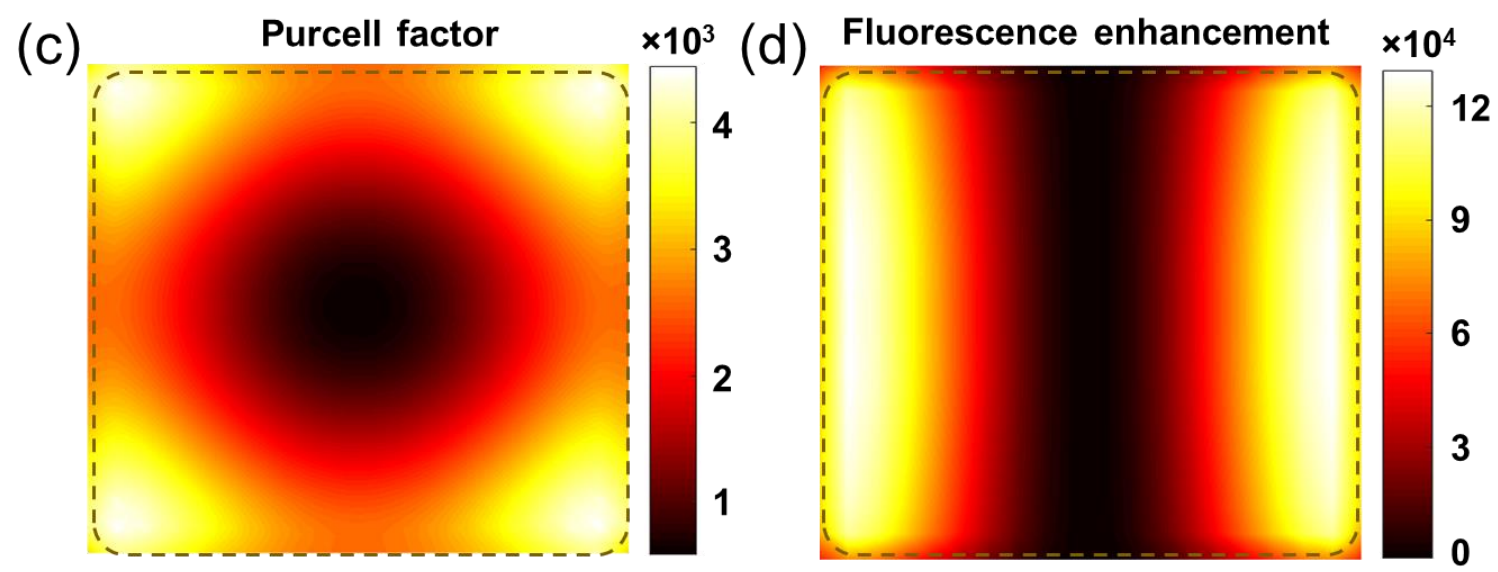

Figure 5.15 (a) Scheme of a point electric dipole at the gap center of the film-coupled Cu nanocube system; (b) quantum efficiency, (c) Purcell factor, and (d) fluorescence enhancement of the plane at the center of the gap (marked as a red dashed line in (a)), calculated at the plasmon resonance wavelength at $\sim 780 \mathrm{~nm}$ as determined in Figure 1 (c), with an intrinsic quantum efficiency $\eta_{0}=0.01$ for the point dipole. 
(a)

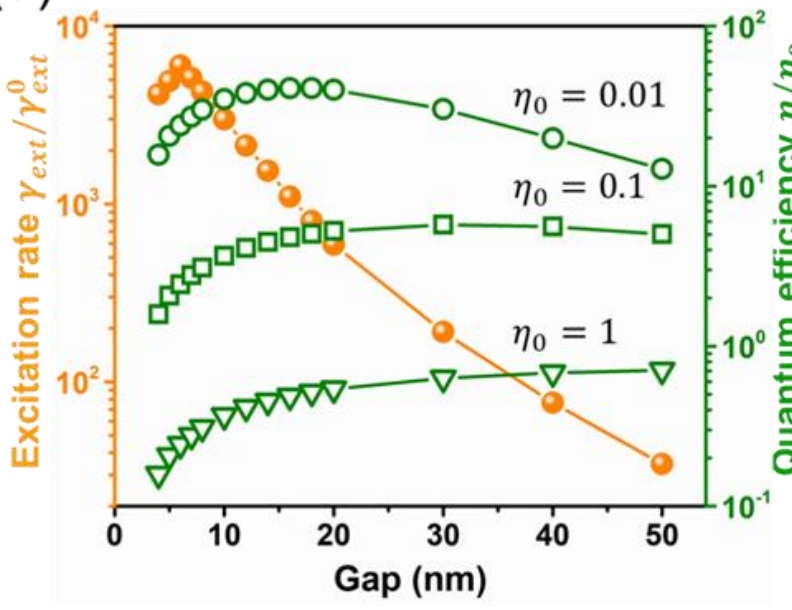

(b)

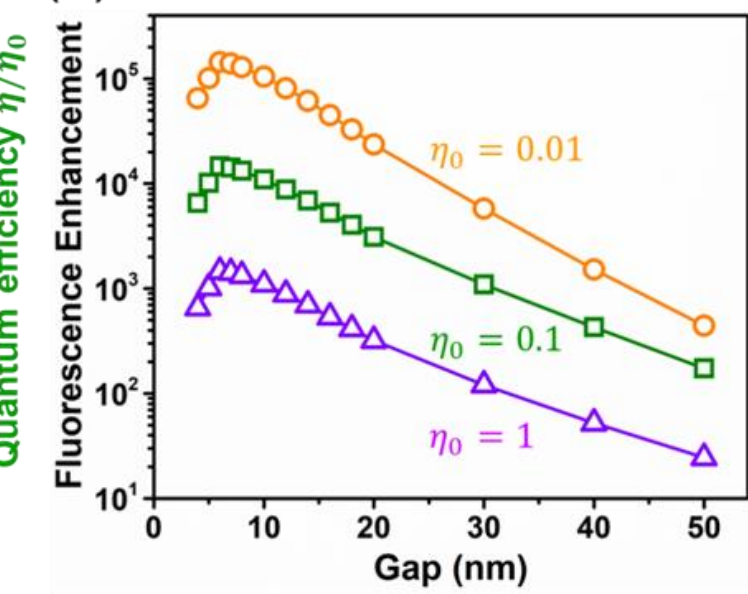

Figure 5.16 Gap-dependent (a) excitation rate enhancement and the quantum efficiency enhancement and (b) the fluorescence enhancement for quantum emitters with intrinsic quantum efficiency of $0.01,0.1$, and 1. The film-coupled Cu nanocube system used is the same as in Figure 5.14

By varying the gap size, the excitation rate enhancement $\left(\gamma_{\text {ext }} / \gamma_{\text {ext }}^{0}\right)$, the quantum efficiency enhancement $\left(\eta / \eta_{0}\right)$, and the resulting fluorescence emission enhancement $\left(\gamma_{e m} / \gamma_{e m}^{0}\right)$ all experienced significant changes, as shown in Figure 5.15. While both the excitation rate and the fluorescence emission enhancement increased exponentially as the gap became smaller, their magnitudes started dropping when the gap shrank less than $6 \mathrm{~nm}$. Given the large gap in quantum scale, it is unlikely that quantum tunneling or nonlocal effects play any role in capping the largest achievable enhancements. ${ }^{28}$ Rather, it is more likely that a less efficient coupling at a smaller gap between the $\mathrm{Cu}$ nanostructure and the incident light leads to the decrease of the excitation rate enhancement; in the meanwhile, a smaller gap facilitates the quantum emitter to couple with higher-order plasmonic cavity modes with the energy dissipating nonradiatively, which compromises the quantum efficiency and finally results in the decrease of the fluorescence emission enhancement. Despite the slight decrease, the film-coupled $\mathrm{Cu}$ nanocube system maintains a high level of enhancement at a smaller gap, as shown in Figure 5.15(b), which has a strong implication that the stringent condition of a delicately-balanced gap in conventional plasmon-enhanced fluorescence can be relaxed.

In addition, the intrinsic quantum efficiency was also varied from 0.01 to 0.1 and 1 to study how it affects the fluorescence enhancement. It turns out that the fluorescence emission of a weaker quantum emitter can be more strongly enhanced as compared to a stronger one, which is consistent with previous reports. ${ }^{29-30}$ This implies that the nature of a low intrinsic quantum efficiency makes it easier for NIR quantum emitters to experience a larger fluorescence emission enhancement 


\subsubsection{Conclusion}

In summary, the film-coupled $\mathrm{Cu}$ nanocube system displays a wide spectral tunability by simply varying the gap thickness. The excited plasmonic cavity mode is accompanied by a strong electric and magnetic field. The localized EM energy oscillates alternatively between the electric and magnetic component and leads a strong modification of the local optical density of states. The NIR quantum emitter placed at the gap region thus experiences a strong enhancement of the excitation rate as well as the quantum efficiency, which results in a large fluorescence emission enhancement. Compared to conventional plasmon-enhanced fluorescence, the film-coupled $\mathrm{Cu}$ nanocube allows the NIR quantum emitter to maintain a high level of enhancement even at small gaps thanks to the plasmonic cavity mode. 


\section{Reference}

1. Inwati, G. K.; Rao, Y.; Singh, M., Thermodynamically induced in Situ and Tunable $\mathrm{Cu}$ Plasmonic Behaviour. Sci Rep-Uk 2018, 8.

2. DeSario, P. A.; Pietron, J. J.; Brintlinger, T. H.; McEntee, M.; Parker, J. F.; Baturina, O.; Stroud, R. M.; Rolison, D. R., Oxidation-stable plasmonic copper nanoparticles in photocatalytic TiO2 nanoarchitectures. Nanoscale 2017, 9 (32), 11720-11729.

3. Chan, G. H.; Zhao, J.; Hicks, E. M.; Schatz, G. C.; Van Duyne, R. P., Plasmonic Properties of Copper Nanoparticles Fabricated by Nanosphere Lithography. Nano Letters 2007, 7 (7), 19471952.

4. Liu, P. S.; Wang, H.; Li, X. M.; Rui, M. C.; Zeng, H. B., Localized surface plasmon resonance of $\mathrm{Cu}$ nanoparticles by laser ablation liquid media. Rsc Advances 2015, 5 (97), 7973879745.

5. Guo, X. N.; Hao, C. H.; Jin, G. Q.; Zhu, H. Y.; Guo, X. Y., Copper Nanoparticles on Graphene Support: An Efficient Photocatalyst for Coupling of Nitroaromatics in Visible Light. Angew Chem Int Edit 2014, 53 (7), 1973-1977.

6. Gunalan, S.; Sivaraj, R.; Venckatesh, R., Aloe barbadensis Miller mediated green synthesis of mono-disperse copper oxide nanoparticles: Optical properties. Spectrochimica Acta Part A: Molecular and Biomolecular Spectroscopy 2012, 97, 1140-1144.

7. Anatoliy, P.; Gero von, P.; Uwe, K., Influence of interband electronic transitions on the optical absorption in metallic nanoparticles. Journal of Physics D: Applied Physics 2004, 37 (22), 3133.

8. Khurgin, J. B., Ultimate limit of field confinement by surface plasmon polaritons. Faraday Discussions 2015, 178 (0), 109-122.

9. Yang, H.-J.; He, S.-Y.; Chen, H.-L.; Tuan, H.-Y., Monodisperse Copper Nanocubes: Synthesis, Self-Assembly, and Large-Area Dense-Packed Films. Chemistry of Materials 2014, 26 (5), 1785-1793.

10. Guo, H. Z.; Chen, Y. Z.; Cortie, M. B.; Liu, X.; Xie, Q. S.; Wang, X.; Peng, D. L., ShapeSelective Formation of Monodisperse Copper Nanospheres and Nanocubes via Disproportionation Reaction Route and Their Optical Properties. J. Phys. Chem. C 2014, 118 (18), 9801-9808.

11. Crane, C. C.; Wang, F.; Li, J.; Tao, J.; Zhu, Y. M.; Chen, J. Y., Synthesis of Copper-Silica Core-Shell Nanostructures with Sharp and Stable Localized Surface Plasmon Resonance. J. Phys. Chem. C 2017, 121 (10), 5684-5692.

12. Fuchs, R., Theory of the optical properties of ionic crystal cubes. Physical Review B 1975, 11 (4), 1732-1740.

13. Byers, C. P.; Zhang, H.; Swearer, D. F.; Yorulmaz, M.; Hoener, B. S.; Huang, D.; Hoggard, A.; Chang, W. S.; Mulvaney, P.; Ringe, E.; Halas, N. J.; Nordlander, P.; Link, S.; Landes, C. F., From tunable core-shell nanoparticles to plasmonic drawbridges: Active control of nanoparticle optical properties. Sci Adv 2015, 1 (11). 
14. Prodan, E.; Radloff, C.; Halas, N. J.; Nordlander, P., A hybridization model for the plasmon response of complex nanostructures. Science 2003, 302 (5644), 419-422.

15. Rose, A.; Hoang, T. B.; McGuire, F.; Mock, J. J.; Ciraci, C.; Smith, D. R.; Mikkelsen, M. H., Control of Radiative Processes Using Tunable Plasmonic Nanopatch Antennas. Nano Letters 2014, 14 (8), 4797-4802.

16. Akselrod, G. M.; Argyropoulos, C.; Hoang, T. B.; Ciraci, C.; Fang, C.; Huang, J. N.; Smith, D. R.; Mikkelsen, M. H., Probing the mechanisms of large Purcell enhancement in plasmonic nanoantennas. Nature Photonics 2014, 8 (11), 835-840.

17. Lassiter, J. B.; McGuire, F.; Mock, J. J.; Ciraci, C.; Hill, R. T.; Wiley, B. J.; Chilkoti, A.; Smith, D. R., Plasmonic Waveguide Modes of Film-Coupled Metallic Nanocubes. Nano Letters 2013, 13 (12), 5866-5872.

18. Moreau, A.; Ciraci, C.; Mock, J. J.; Hill, R. T.; Wang, Q.; Wiley, B. J.; Chilkoti, A.; Smith, D. R., Controlled-reflectance surfaces with film-coupled colloidal nanoantennas. Nature 2012, 492 (7427), 86-+.

19. Chen, W.; Zhang, S. P.; Deng, Q.; Xu, H. X., Probing of sub-picometer vertical differential resolutions using cavity plasmons. Nat Commun 2018, 9.

20. Tan, S. F.; Wu, L.; Yang, J. K. W.; Bai, P.; Bosman, M.; Nijhuis, C. A., Quantum Plasmon Resonances Controlled by Molecular Tunnel Junctions. Science 2014, 343 (6178), 1496-1499.

21. Knebl, D.; Horl, A.; Trugler, A.; Kern, J.; Krenn, J. R.; Puschnig, P.; Hohenester, U., Gap plasmonics of silver nanocube dimers. Physical Review B 2016, 93 (8).

22. Zhu, W. Q.; Esteban, R.; Borisov, A. G.; Baumberg, J. J.; Nordlander, P.; Lezec, H. J.; Aizpurua, J.; Crozier, K. B., Quantum mechanical effects in plasmonic structures with subnanometre gaps. Nat Commun 2016, 7.

23. Shcherbakova, D. M.; Baloban, M.; Emelyanov, A. V.; Brenowitz, M.; Guo, P.; Verkhusha, V. V., Bright monomeric near-infrared fluorescent proteins as tags and biosensors for multiscale imaging. Nat Commun 2016, 7.

24. Johnson, P. B.; Christy, R. W., Optical Constants of the Noble Metals. Physical Review B 1972, 6 (12), 4370-4379.

25. Zangwill, A., Modern Electrodynamics. Cambridge University Press: 2013.

26. Popović, Z. B.; Popović, B. D., Introductory Electromagnetics. Prentice Hall: 2000.

27. Anger, P.; Bharadwaj, P.; Novotny, L., Enhancement and quenching of single-molecule fluorescence. Phys Rev Lett 2006, 96 (11).

28. Esteban, R.; Borisov, A. G.; Nordlander, P.; Aizpurua, J., Bridging quantum and classical plasmonics with a quantum-corrected model. Nat Commun 2012, 3.

29. Sun, G.; Khurgin, J. B., Origin of giant difference between fluorescence, resonance, and nonresonance Raman scattering enhancement by surface plasmons. Phys Rev A 2012, 85 (6), 063410.

30. Khurgin, J. B.; Sun, G., Enhancement of optical properties of nanoscaled objects by metal nanoparticles. J Opt Soc Am B 2009, 26 (12), B83-B95. 


\section{Chapter 6: Outlook and Conclusions}

\subsection{Outlook: Atom-Light Interaction at the Strong-Coupling Limit}
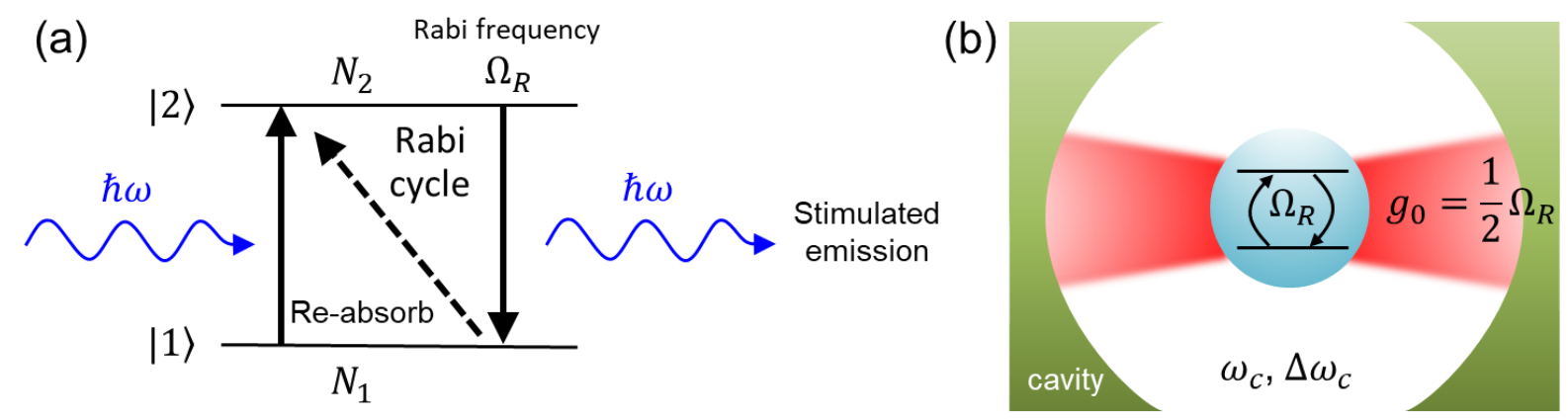

Figure 6.1 (a) Rabi oscillations at the strong-field limit; (b) Atom-cavity interaction at the strong coupling limit.

In the strong-field limit, the resonant atom-light interaction results in coherent superposition states $|\psi\rangle=c_{1}|1\rangle+c_{2}|2\rangle$, where $c_{1}$ and $c_{2}$ are the normalized coefficients representing the probability of finding the atom in the corresponding state. The coherent superposition states are characterized by the Rabi frequency $\Omega_{R}$, which exceeds the spontaneous decay rate and quantifies the number of cycles the atom oscillates between the excited and ground state per unit time under the stimulation of the incident field. As more electrons build up at the excited state $|2\rangle$ under strong incident field, population inversion would finally occur as the excited population $N_{2}$ would exceed the ground state population $N_{1}$ at some point, which gives rise to stimulated emission. Stimulated emission is the basis for LASER (light amplification by stimulated emission of radiation) and SPASER (surface plasmon amplification by stimulated emission of radiation).

SPASER is the plasmon counterpart of LASER where the incident light is replaced by surface plasmon and the resonant optical cavity is replaced by a plasmonic nanoparticle. It often adopts the nanoparticle-gain material core-shell nanostructure where the nanoparticle supports a surface plasmon mode and the gain material provides the energy source. For a typical SPASER to operate, the gain material is first excited externally regardless of the excitation source. The relaxation of the excited excitonic energy would be transferred to the nanoparticle and excites the surface plasmon. In return, the excited surface plasmon would further stimulate excitation of excitons in the gain material, which ends up exciting more identical surface plasmons. This process repeats itself until the threshold for stimulated emission is reached. 
The reason why surface plasmon could prevail in SPASER is because of its electric neutrality and boson nature with spin 1 , which is the same as photons. Its highly coherent nature also makes it possible to accumulate a large number of surface plasmons in a single mode. Therefore, when the population inversion is achieved, stimulated emission could occur, generating a highly coherent, intense, and ultrafast emission, which is called spasing. Given the nanoscale nature of all the elements involved, SPASER could operate as a nanoscale optoelectronic device and holds great promise for ultramicroscopy and other nanodevices.

Compared to fluorescence, SPASER and PEF share some similarities but also differ from each other significantly.

First, both the SPASER probe and PEF probe could be constructed as a core-shell nanoparticle to support an enhanced optical emission. Both need a plasmonic nanoparticle the provide the driving force and an exciton-like material to provide the light source.

Second, they differ drastically in the emission profile. While PEF displays a Stokes-shifted emission spectrum with a broadened linewidth due to the long fluorescence lifetime, SPASER supports an emission spectrum free of Stokes shift and with a very sharp linewidth due to the ultrafast emission nature.

However, in order to achieve the threshold for spasing, the incident light source is usually pulsed laser, which could be a potential issue for biological samples, although efforts are under way to mitigate the laser-induced damage to biological samples. In contrast, near-infrared light source could be used in PEF and is well coupled with biological matrix.

Since the stimulated emission is highly coherent and inherits all the features from the incident field, it would be interesting to see how stimulated emission could be coupled with fluorescence emission under the influence of surface plasmon resonance.

In the meanwhile, when the atom-cavity coupling strength, which is defined as $g_{0}=\frac{1}{2} \Omega_{R}$, outcompetes any other energy loss rates in the system, the strong-coupling regime would be reached. In the strong-coupling limit, the incident energy would be trapped and oscillates between the optical cavity and the atom. The consequence is that the optical cavity mode and the excited atomic state would be hybridized, ending up producing two new plexcitonic states, which are halflight and half-matter. The plexcitonic states are essentially of quantum nature and have strong implications in ultra-efficient light emission, nonclassical or squeezed state generation, emission of correlated photons, etc. By placing the atom-cavity system to interact in the strong-coupling regime, it would be very interesting to see how surface-enhanced spectroscopy could be benefited from the plexcitonic states. 


\subsection{Conclusions}

In this dissertation, surface-enhanced spectroscopy has been studied for optical sensing and the prospect of acting as preventative measures in dealing with environment, food, and healthcare related issues has been assessed.

SERS and PEF as two parallel routes have been applied to build proof-of-concept sensors. SERS sensors are featured by the simplicity in SERS signal optimization thanks to the fourth power rule, which states that the SERS enhancement scales with the fourth power of the local EM field enhancement. In comparison, PEF sensors need a delicate balance between the excitation enhancement and emission enhancement as they have different distance dependence. While it appears that SERS method is promising for POC sensor devices, it is noted that the laser beam in Raman spectroscopy needs to be delicately focused to achieve high SERS spectral resolution. This is probably a major drawback for SERS-based POC sensor devices. While it is true that a lot of efforts need to be devoted to balance the excitation and emission enhancement in fluorescence, the emission nature of fluorescence makes it easily coupled with POC fluorescence readers, as demonstrated by the PEF biosensors for TBI biomarker detection. This gives fluorescence a huge leverage.

In addition to exploiting noble metals such as gold and silver to study surface-enhanced spectroscopy, alternative cost-effective plasmonic materials have also been explored. In this dissertation, $\mathrm{Cu}$ nanocubes have been found to support strong LSPR, which has an origin of the plasmonic corner modes. Compared to the dipole mode of Au nanospheres of the same effective radius, $\mathrm{Cu}$ nanocubes have a comparable extinction coefficient but much larger local EM field. The results strongly indicate copper is a highly promising alternative inexpensive plasmonic materials.

In conclusion, the knowledge obtained in studying SERS and PEF lays out a technical foundation for future transformation of proof-of-concept sensors into POC sensor devices. The discovery of copper as a cheap plasmonic metal further makes POC optical sensing a foreseeable goal. 


\section{Appendix}

\section{Manuscripts}

1. Film-Coupled Copper Nanocube for Near-Infrared Fluorescence Enhancement Peng Zheng, Sujan Kasani, Nianqiang Wu

2. Near-Infrared Fluorescent Detection of Glial Fibrillary Acidic Protein in Blood Plasma Based on Gold Nano-pyramid Array

Peng Zheng, Sujan Kasani, Xuefei Gao, Kathrine Curtin, Nianqiang Wu

3. Elucidating the Role of Scattering in Plasmon-Enhanced Fluorescence Peng Zheng, Botong Liu, Sujan Kasani, Haibin Tang, Nianqiang Wu

4. Metal-Organic Framework Coated Titanium Dioxide Nanorod Array p-n Heterojunction Photoanode for Photoelectrochemical Water-Splitting Hui Yang, Joeseph Bright, Sujan Kasani, Peng Zheng, Terence Musho, Banglin Chen, Ling Huang, Nianqiang $\mathrm{Wu}$

\section{Publications ( ${ }^{*}$ Co-first authorship)}

1. Converting Plasmonic Light Scattering to Confined Light Absorption and Creating Plexcitons by Coupling a Gold Nano-pyramid Array to a Silica-Gold Film Nanoscale Horizons, 2018, DOI: 10.1039/C8NH00286J

Peng Zheng, ${ }^{*}$ Sujan Kasani,* Nianqiang Wu

2. Origin of Strong and Narrow Localized Surface Plasmon Resonance of Copper Nanocubes

Nano Research, 2018, doi.org/10.1007/s12274-018-2178-6

Peng Zheng, * Haibin Tang,* Botong Liu, Sujan Kasani, Ling Huang, Nianqiang Wu

3. Detection of Nitrite with a Surface-Enhanced Raman Scattering Sensor Based on Silver Nanopyramid Array Analytica Chimica Acta, 2018, 1040, 158-165

Peng Zheng, Sujan Kasani, Xiaofei Shi, Haibin Tang, Ming Li, Ashley E. Boryczka, Wanhong Zheng, Daniel E. Elswick, Feng Yang, Nianqiang Wu

4. Tailoring Optical Properties of a Large-Area Plasmonic Gold Nano-Ring Array Pattern

The Journal of Physical Chemistry C, 2018, 122, 13443-13449

Sujan Kasani,* Peng Zheng, * Nianqiang Wu 
5. Fluorescence and Sensing Applications of Graphene Oxide and Graphene Quantum Dots: A Review

Chemistry - An Asian Journal, 2017, 12, 2343-2353

Peng Zheng, Nianqiang $\mathrm{Wu}$

6. Detection of Mercury (II) with a Surface-Enhanced Raman Scattering Sensor Based on Functionalized Gold Nanoparticles

Materials Research Express, 2017, 4, 055017

Peng Zheng, Xiaofei Shi, Kathrine Curtin, Feng Yang, Nianqiang Wu

7. A Gold Nanohole Array Based Surface-Enhanced Raman Scattering (SERS)

Biosensor for Detection of Silver (I) and Mercury (II) in Human Saliva

Nanoscale, 2015, 7, 11005-11012

Peng Zheng, Ming Li, Richard Jurevic, Scott K. Cushing, Yuxin Liu, Nianqiang Wu

8. Tailoring Plasmonic Properties of Gold Nanohole Arrays for Surface-Enhanced Raman Scattering

Physical Chemistry Chemical Physics, 2015, 17, 21211-21219

Peng Zheng, Scott K. Cushing, Savan Suri, Nianqiang Wu

9. Paper-Based Surface-Enhanced Raman Scattering Lateral Flow Strip for Detection of Neuron-Specific Enolase in Blood Plasma

Analytical Chemistry, 2017, 89, 10104-10110

Xuefei Gao, Peng Zheng, Sujan Kasani, Steven Wu, Feng Yang, Sara Lewis, Sara Nayeem, Elizabeth B. Engler-Chiurazzi, Jane G. Wigginton, James W. Simpkins, Nianqiang $\mathrm{Wu}$

10. Effect of Surface Functionalizations of Multi-walled Carbon Nanotubes on Neoplastic Transformation Potential in Primary Human Lung Epithelial Cells Nanotoxicology, 2017, 11, 613-624

Todd A Stueckle, Donna C Davidson, Ray Derk, Peng Wang, Sherri Friend, Diane Schwegler-Berry, Peng Zheng, Nianqiang Wu, Vince Castranova, Yon Rojanasakul, Liying Wang

11. Lung Bioactivity of Vapor Grown Carbon Nanofibers

NanoImpact, 2017, 6, 1-10

Dale W Porter, Marlene Orandle, Robert R Mercer, Nianqiang Wu, Peng Zheng, Bean T Chen, Andrij Holian, Michael Andrew, Stephen Leonard, Michael Wolfarth, Sherri Friend, Lori Battelli, Raymond F Hamilton, Yuji Hagiwara, Tamami Koyama, Vincent Castranova

12. Effects of Defects on Photocatalytic Activity of Hydrogen-Treated Titanium Oxide Nanobelts ACS Catalysis, 2017, 7, 1742-1748

Scott K Cushing, Fanke Meng, Junying Zhang, Bangfu Ding, Chih Kai Chen, ChihJung Chen, Ru-Shi Liu, Alan D Bristow, Joeseph Bright, Peng Zheng, Nianqiang Wu 
13. An Ordered Array of Hierarchical Spheres for Surface-Enhanced Raman Scattering Detection of Trace Pesticide

Nanotechnology, 2016, 27, 384001

Xiaoye Hu, Peng Zheng, Guowen Meng, Qing Huang, Chuhong Zhu, Fangming Han, Zhulin Huang, Zhongbo Li, zhaoming wang, Nianqiang Wu

14. Fabrication of Hexagonally Patterned Flower-like Silver Particle Arrays as Surface-enhanced Raman Scattering Substrates

Nanotechnology, 2016, 27, 325303

Haibin Tang, Peng Zheng, Guowen Meng, Zhongbo Li, Chuhong Zhu, Fangming

Han, Yan Ke, Zhaoming Wang, Fei Zhou, Nianqiang Wu

15. Distinguishing Surface Effects of Gold Nanoparticles from Plasmonic Effect on Photoelectrochemical Water Splitting by Hematite Journal of Materials Research, 2016, 31, 1608-1615

Jiangtian Li, Scott K Cushing, Deryn Chu, Peng Zheng, Joeseph Bright, Conner Castle, Ayyakkannu Manivannan, Nianqiang Wu

16. A Hierarchically Ordered Array of Silver-Nanorod Bundles for Surface-Enhanced Raman Scattering Detection of Phenolic Pollutants

Advanced Materials, 2016, 28, 4871-4876

Chuhong Zhu, Guowen Meng, Peng Zheng, Qing Huang, Zhongbo Li, Xiaoye Hu, Xiujuan Wang, Zhulin Huang, Fadi Li, Nianqiang Wu

17. Investigation of the Plasmonic Effect in Air-processed PbS/CdS Core-shell Quantum Dot Based Solar Cells Journal of Materials Chemistry A, 2016, 4, 13071-13080

Belete Atomsa Gonfa, Mee Rahn Kim, Peng Zheng, Scott Cushing, Qiquan Qiao, Nianqiang Wu, My Ali El Khakani, Dongling Ma

18. Controlling Plasmon-induced Resonance Energy Transfer and Hot Electron Injection Processes in Metal@ TiO2 Core-shell Nanoparticles Journal of Physical Chemistry C, 2015, 119, 16239-16244

Scott K Cushing, Jiangtian Li, Joeseph Bright, Brandon T Yost, Peng Zheng, Alan D Bristow, Nianqiang $\mathrm{Wu}$

19. A Surface-enhanced Raman Scattering Sensor Integrated with Battery-controlled Fluidic Device for Capture and Detection of Trace Small Molecules Scientific Reports, 2015, 5, 12865

Qitao Zhou, Guowen Meng, Peng Zheng, Scott Cushing, Nianqiang Wu, Qing Huang, Chuhong Zhu, Zhuo Zhang, Zhiwei Wang

20. Solar Hydrogen Generation by a CdS-Au-TiO2 Sandwich Nanorod Array Enhanced with Au Nanoparticle as Electron Relay and Plasmonic Photosensitizer Journal of the American Chemical Society, 2014, 136, 8438-8449 Jiangtian Li, Scott K Cushing, Peng Zheng, Tess Senty, Fanke Meng, Alan D Bristow, Ayyakkannu Manivannan, Nianqiang Wu 
21. A Gold@ Silica Core-shell Nanoparticle-based Surface-enhanced Raman Scattering Biosensor for Label-free Glucose Detection

Analytica chimica acta, 2014, 811, 76-80 Israa Al-Ogaidi, Honglei Gou, Abdul Kareem A Al-Kazaz, Zoraida P Aguilar, Alice K Melconian, Peng Zheng, Nianqiang Wu

22. Plasmon-induced Photonic and Energy Transfer Enhancement of Solar Water Splitting by a Hematite Nanorod Array Nature Communications. 2014, 4, 2651 Jiangtian Li, Scott K. Cushing, Peng Zheng, Fanke Meng, Deryn Chu, Nianqiang Wu 23. Ag@ Cu2O Core-shell Nanoparticles as Visible-light Plasmonic Photocatalysts ACS Catalysis, 2012, 3, 47-51 Jiangtian Li, Scott K Cushing, Joeseph Bright, Fanke Meng, Tess R Senty, Peng Zheng, Alan D Bristow, Nianqiang Wu 